\title{
Regular and irregular holonomic D-modules
}

\author{
Masaki Kashiwara and Pierre Schapira
}

July 1, 2015

\begin{abstract}
This is a survey paper. In a first part, we recall the main results on the tempered holomorphic solutions of D-modules in the language of indsheaves and, as an application, the Riemann-Hilbert correspondence for regular holonomic modules. In a second part, we present the enhanced version of the first part, treating along the same lines the irregular holonomic case.
\end{abstract}

\section{Contents}

$\begin{array}{lr}\text { Introduction } & 3\end{array}$

1 A brief review on sheaves and D-modules $\quad 7$

1.1 Sheaves ........................ 7

1.2 D-modules . . . . . . . . . . . . . . . . . . . 10

2 Indsheaves 15

2.1 Ind-objects . . . . . . . . . . . . . . . . 15

2.2 Indsheaves . . . . . . . . . . . . . . . . . . . . 17

2.3 Ring action ................... . . 20

2.4 Sheaves on the subanalytic site . . . . . . . . . . . 24

2.5 Some classical sheaves on the subanalytic site . . . . . . . . 28

2.5.1 Tempered and Whitney functions and distributions . . 29

2.5.2 Operations on tempered distributions . . . . . . . 31

2.5.3 Whitney and tempered holomorphic functions . . . . . 34

2.5.4 Duality between Whitney and tempered functions . . 35 
3 Tempered solutions $\quad 38$

3.1 Tempered de Rham and Sol functors . . . . . . . . . . . . 38

3.2 Localization along a hypersurface . . . . . . . . . . . . . . 42

4 Regular holonomic D-modules 44

4.1 Regular normal form for holonomic modules . . . . . . . . . . 44

4.2 Real blow up . . . . . . . . . . . . . . . . . 46

4.3 Regular Riemann-Hilbert correspondence . . . . . . . . . . . . 49

4.4 Integral transforms with regular kernels . . . . . . . . . . . . 54

4.5 Irregular D-modules: an example . . . . . . . . . . . . . . 55

5 Indsheaves on bordered spaces $\quad 56$

5.1 Bordered spaces . . . . . . . . . . . . . . . 56

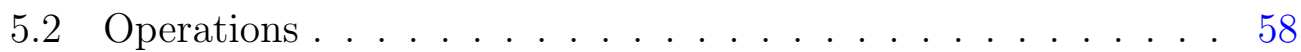

6 Enhanced indsheaves $\quad 61$

6.1 Tamarkin's construction . . . . . . . . . . . . . . 61

6.2 Convolution products . . . . . . . . . . . . 62

6.3 Enhanced indsheaves ................ . . 65

6.4 Operations on enhanced indsheaves . . . . . . . . . . . 68

6.5 Stable objects . . . . . . . . . . . . . . . . 71

6.6 Constructible enhanced indsheaves . . . . . . . . . . 73

6.7 Enhanced indsheaves with ring action . . . . . . . . . . . 77

7 Holonomic D-modules $\quad 77$

7.1 Exponential D-modules . . . . . . . . . . . . . . 77

7.2 Enhanced tempered holomorphic functions . . . . . . . . . 78

7.3 Enhanced de Rham and Sol functors . . . . . . . . . . . . . 80

7.4 Ordinary linear differential equations and Stokes phenomena . 81

7.5 Normal form . . . . . . . . . . . . . . . . . . . . . . 85

7.6 Enhanced de Rham functor on the real blow up . . . . . . . . 87

7.7 De Rham functor: constructibility and duality . . . . . . . . . 88

7.8 Enhanced Riemann-Hilbert correspondence . . . . . . . . . . . 94

$\begin{array}{llr}8 & \text { Integral transforms } & 97\end{array}$

8.1 Integral transforms with irregular kernels . . . . . . . . . 97

8.2 Enhanced Fourier-Sato transform . . . . . . . . . . . . . . 98

8.3 Laplace transform . . . . . . . . . . . . . . . . . . . . . . 100 
List of Notations 109

$\begin{array}{ll}\text { Index } & 112\end{array}$

\section{Introduction}

These Notes are an expanded version of a series of lectures given at the IHES in February/March 2015 (see [KS 15]), based on [DK 13] and [KS 14].

Here, we assume the reader familiar with the language of sheaves and D-modules, in the derived sense.

Let $X$ be a complex manifold. Denote by $\operatorname{Mod}\left(\mathscr{D}_{X}\right)$ the abelian category of left $\mathscr{D}_{X}$-modules, by $\operatorname{Mod}_{\text {hol }}\left(\mathscr{D}_{X}\right)$ the full subcategory of holonomic $\mathscr{D}_{X^{-}}$ modules and by $\operatorname{Perv}\left(\mathbb{C}_{X}\right)$ the abelian category of perverse sheaves with coefficients in $\mathbb{C}$. Consider the functor constructed in [Ka 75]

$$
\begin{aligned}
& \text { Sol: } \operatorname{Mod}_{\text {hol }}\left(\mathscr{D}_{X}\right)^{\mathrm{op}} \rightarrow \operatorname{Perv}\left(\mathbb{C}_{X}\right), \\
& \mathscr{M} \mapsto \operatorname{R} \mathscr{H o m}_{\mathscr{D}}\left(\mathscr{M}, \mathscr{O}_{X}\right) .
\end{aligned}
$$

(Note that at this time the notion of perverse sheaves was not explicit, but in his paper the author proved that $\mathrm{R} \mathscr{H} o m_{\mathscr{D}}\left(\mathscr{M}, \mathscr{O}_{X}\right)$ is $\mathbb{C}$-constructible and satisfies the properties which are now called perversity.)

It is well-known that this functor is not faithful. For example, if $X=$ $\mathbb{A}^{1}(\mathbb{C})$, the complex line with coordinate $t, P=t^{2} \partial_{t}-1$ and $Q=t^{2} \partial_{t}+t$, then the two $\mathscr{D}_{X}$-modules $\mathscr{D}_{X} / \mathscr{D}_{X} P$ and $\mathscr{D}_{X} / \mathscr{D}_{X} Q$ have the same sheaves of solutions.

A natural idea to overcome this difficulty is to replace the sheaf $\mathscr{O}_{X}$ with presheaves of holomorphic functions with various growths such as for example the presheaf $\mathscr{O}_{X}^{\mathrm{t}}$ of holomorphic functions with tempered growth. This presheaf is not a sheaf for the usual topology, but it becomes a sheaf for a suitable Grothendieck topology, the subanalytic topology, and here we shall embed the category of subanalytic sheaves in that of indsheaves.

As we shall see, the indsheaf $\mathscr{O}_{X}^{\mathrm{t}}$ is not sufficient to obtain a RiemannHilbert correspondence, but it is a first step to this direction. To obtain a final result, it is necessary to add an extra variable and to work with an "enhanced" version of $\mathscr{O}_{X}^{\mathrm{t}}$ in order to describe "various growths" in a rigorous way.

In a first part, we shall recall the main results of the theory of indsheaves and subanalytic sheaves and we shall explain with some details the 
operations on D-modules and their tempered holomorphic solutions. As an application, we obtain the Riemann-Hilbert correspondence for regular holonomic D-modules as well as the fact that the de Rham functor commutes with integral transforms.

In a second part, we do the same for the sheaf of enhanced tempered solutions of (no more necessarily regular) holonomic D-modules. For that purpose, we first recall the main results of the theory of indsheaves on bordered spaces and its enhanced version, a generalization to indsheaves of a construction of Tamarkin [Ta 08].

Let us describe with some details the contents of these Notes.

Section 1 is a brief review on the theory of sheaves and D-modules. Its aim is essentially to fix the notations and to recall the main formulas of constant use.

In Section 2, extracted from [KS 96, KS 01], we briefly describe the category of indsheaves on a locally compact space and the six operations on indsheaves. A method for constructing indsheaves on a subanalytic space is the use of the subanalytic Grothendieck topology, a topology for which the open sets are the open relatively compact subanalytic subsets and the coverings are the finite coverings. On a real analytic manifold $M$, this allows us to construct the indsheaves of Whitney functions, tempered $\mathrm{C}^{\infty}$-functions and tempered distributions. On a complex manifold $X$, by taking the Dolbeault complexes with such coefficients, we obtain the indsheaf (in the derived sense) $\mathscr{O}_{X}^{\mathrm{w}}$ of Whitney holomorphic functions and the indsheaf $\mathscr{O}_{X}^{\mathrm{t}}$ of tempered holomorphic functions.

Then, in Section 3, also extracted from [KS96, KS01], we study the tempered de Rham and Sol (Sol for solutions) functors, that is, we study these functors with values in the sheaf of tempered holomorphic functions. We prove two main results which will be the main tools to treat the regular Riemann-Hilbert correspondence later. The first one is Theorem 3.1.1 which calculates the inverse image of the tempered de Rham complex. It is a reformulation of a theorem of [Ka84], a vast generalization of the famous Grothendieck theorem on the de Rham cohomology of algebraic varieties. The second result, Theorem 3.1.5, is a tempered version of the Grauert direct image theorem.

In Section 4 we give a proof of the main theorem of [Ka80, Ka 84] on the Riemann-Hilbert correspondence for regular holonomic D-modules (see 
Corollary 4.3.4). Our proof is based on Lemma 4.1.3 which essentially claims that to prove that regular holonomic D-modules have a certain property, it is enough to check that this property is stable by projective direct images and is satisfied by modules of "regular normal forms", that is, modules associated with equations of the type $z_{i} \partial_{z_{i}}-\lambda_{i}$ or $\partial_{z_{j}}$. The Riemann-Hilbert correspondence as formulated in loc. cit. is not enough to treat integral transform and we have to prove a "tempered" version of it (Theorem 4.3.2). We then collect all results on the tempered solutions of D-modules in a single formula which, roughly speaking, asserts that the tempered de Rham functor commutes with integral transforms whose kernel is regular holonomic (Theorem 4.4.2). We end this section with a detailed study of the irregular holonomic D-module $\mathscr{D}_{X} \exp (1 / z)$ on $\mathbb{A}^{1}(\mathbb{C})$, following $[\mathrm{KS} 03]$. This case shows that the solution functor with values in the indsheaf $\mathscr{O}_{X}^{\mathrm{t}}$ gives many informations on the holonomic D-modules, but not enough: it is not fully faithful. As seen in the next sections, in order to treat irregular case, we need the enhanced version of the setting discussed in this section.

Section 5, extracted from [DK 13], treats indsheaves on bordered spaces. A bordered space is a pair $(M, \widehat{M})$ of good topological spaces with $M \subset \widehat{M}$ an open embedding. The derived category of indsheaves on $(M, \widehat{M})$ is the quotient of the category of indsheaves on $\widehat{M}$ by that of indsheaves on $\widehat{M} \backslash M$. Indeed, contrarily to the case of usual sheaves, this quotient is not equivalent to the derived category of indsheaves on $M$.

The main idea to treat the irregular Riemann-Hilbert correspondence is to replace the indsheaf $\mathscr{O}_{X}^{\mathrm{t}}$ with an enhanced version, the object $\mathscr{O}_{X}^{\mathrm{E}}$. Roughly speaking, this object (which is no more an indsheaf) is obtained as the image of the complex of solutions of the operator $\partial_{t}-1$ acting on $\mathscr{O}_{X \times \mathbb{C}}^{\mathrm{t}}$, in a suitable category, namely that of enhanced indsheaves.

Section 6, also extracted from [DK13], defines and studies the triangulated category $\mathrm{E}^{\mathrm{b}}\left(\mathrm{Ik}_{M}\right)$ of enhanced indsheaves on $M$, adapting to indsheaves a construction of Tamarkin [Ta 08]. Denoting by $\mathbb{R}_{\infty}$ the bordered space $(\mathbb{R}, \overline{\mathbb{R}})$ in which $\overline{\mathbb{R}}$ is the two points compactification of $\mathbb{R}$, the category $\mathrm{E}^{\mathrm{b}}\left(\mathrm{Ik}_{M}\right)$ is the quotient of the category of indsheaves on $M \times \mathbb{R}_{\infty}$ by the subcategory of indsheaves which are isomorphic to the inverse image of indsheaves on $M$.

Section 7, mainly extracted from [DK13], treats the irregular RiemannHilbert correspondence. Similarly as in the regular case, an essential tool is Lemma 7.5.5 which asserts that to prove that holonomic D-modules have a 
certain property, it is enough to check that this property is stable by projective direct images and is satisfied by modules of "normal forms", that is, D-modules of the type $\mathscr{D}_{X} \exp \varphi$ where $\varphi$ is a meromorphic function. This lemma follows directly from the fundamental results of Mochizuki [Mo09, Mo 11] (in the algebraic setting) and later Kedlaya [Ke10, Ke11] in the analytic case, after preliminary results by Sabbah [Sa00]. The proof of the irregular Riemann-Hilbert correspondence is rather intricate and uses enhanced constructible sheaves and a duality result between the enhanced solution functor and the enhanced de Rham functor. However, this theorem formulated in [DK 13] (Corollary 7.8.3) is not enough to treat irregular integral transform and we have to prove an "enhanced" version of it (Theorem 7.8.1, extracted from [KS14]).

In Section 8, extracted from [KS14], we apply the preceding results. The main formula (8.1.4) asserts, roughly speaking, that the enhanced de Rham functor commutes with integral transforms with irregular kernels. In a previous paper [KS 97] we had already proved (without the machinery of enhanced indsheaves) that given a complex vector space $\mathbb{V}$, the Laplace transform induces an isomorphism of the Fourier-Sato transform of the conic sheaf associated with $\mathscr{O}_{\mathbb{V}}^{\mathrm{t}}$ with the similar sheaf on $\mathbb{V}^{*}$ (up to a shift). We obtain here a similar result in a non-conic setting, replacing $\mathscr{O}_{\mathbb{V}}^{\mathrm{t}}$ with its enhanced version $\mathscr{O}_{\mathbb{V}}^{\mathrm{E}}$. For that purpose, we extend first the Tamarkin non conic Fourier-Sato transform to the enhanced setting.

Bibliographical and historical comments. A first important step in a modern treatment of the Riemann-Hilbert correspondence is the book of Deligne [De70]. A second important step is the constructibility theorem [Ka 75] and a precise formulation of this correspondence in 1977 by the same author (see [Ra 78, p. 287]). Then a detailed sketch of proof of the theorem establishing this correspondence (in the regular case) appeared in [Ka 80] where the functor Thom of tempered cohomology was introduced, and a detailed proof appeared in [Ka84]. A different proof to this correspondence appeared in [Me 84]. The functorial operations on the functor Thom, as well as its dual notion, the Whitney tensor product $\stackrel{\mathrm{w}}{\otimes}$, are systematically studied in [KS96]. These two functors are in fact better understood by the language of $\mathscr{O}_{X}^{\mathrm{t}}$ and $\mathscr{O}_{X}^{\mathrm{w}}$, the indsheaves of tempered holomorphic functions and Whitney holomorphic functions introduced in [KS01].

In the early 2000, it became clear that the indsheaf $\mathscr{O}_{X}^{\mathrm{t}}$ of tempered holomorphic functions is an essential tool for the study of irregular holonomic 
modules and a toy model was studied in [KS03]. However, on $X=\mathbb{A}^{1}(\mathbb{C})$, the two holonomic $\mathscr{D}_{X}$-modules $\mathscr{D}_{X} \exp (1 / t)$ and $\mathscr{D}_{X} \exp (2 / t)$ have the same tempered holomorphic solutions, which shows that $\mathscr{O}_{X}^{\mathrm{t}}$ is not precise enough to treat irregular holonomic D-modules. This difficulty is overcome in [DK 13] by adding an extra variable in order to capture the growth at singular points. This is done, first by adapting to indsheaves a construction of Tamarkin [Ta08], leading to the notion of "enhanced indsheaves", then by defining the "enhanced indsheaf of tempered holomorphic functions". Using fundamental results of Mochizuki [Mo 09, Mo11] (see also Sabbah [Sa 00] for preliminary results and see Kedlaya [Ke10, Ke11] for the analytic case), this leads to the solution of the Riemann-Hilbert correspondence for (not necessarily regular) holonomic D-modules.

As already mentioned, most of the results discussed here are already known. We sometimes don't give proofs, or only give a sketch of the proof. However, Theorems 2.5.13, 6.6.4 and Corollaries 2.5.15, 7.7.2 are new.

\section{A brief review on sheaves and D-modules}

As already mentioned in the introduction, we assume the reader familiar with the language of sheaves and D-modules, in the derived sense. Hence, the aim of this section is mainly to fix some notations.

\section{$1.1 \quad$ Sheaves}

We refer to [KS 90] for all notions of sheaf theory used here. For simplicity, we denote by $\mathbf{k}$ a field, although most of the results would remain true when $\mathbf{k}$ is a commutative ring of finite global dimension.

A topological space is good if it is Hausdorff, locally compact, countable at infinity and has finite flabby dimension. Let $M$ be such a space. For a subset $A \subset M$, we denote by $\bar{A}$ its closure and $\operatorname{Int}(A)$ its interior.

One denotes by $\operatorname{Mod}\left(\mathbf{k}_{M}\right)$ the abelian category of sheaves of $\mathbf{k}$-modules on $M$ and by $\mathrm{D}^{\mathrm{b}}\left(\mathbf{k}_{M}\right)$ its bounded derived category. Note that $\operatorname{Mod}\left(\mathbf{k}_{M}\right)$ has a finite homological dimension.

For a locally closed subset $A$ of $M$, one denotes by $\mathbf{k}_{A}$ the constant sheaf on $A$ with stalk $\mathbf{k}$ extended by 0 on $X \backslash A$. For $F \in \mathrm{D}^{\mathrm{b}}\left(\mathbf{k}_{M}\right)$, one sets $F_{A}:=F \otimes \mathbf{k}_{A}$. One denotes by $\operatorname{Supp}(F)$ the support of $F$. 


\subsection{Sheaves}

We shall make use of the dualizing complex on $M$, denoted by $\omega_{M}$, and the duality functors

$$
\mathrm{D}_{M}^{\prime}:=\mathrm{R} \mathscr{H} o m\left(\cdot, \mathbf{k}_{M}\right), \quad \mathrm{D}_{M}:=\mathrm{R} \mathscr{H} o m\left(\bullet, \omega_{M}\right) .
$$

Recall that, when $M$ is a real manifold, $\omega_{M}$ is isomorphic to the orientation sheaf shifted by the dimension.

We have the two internal operations of internal hom and tensor product:

$$
\begin{array}{rll}
\mathrm{R} \mathscr{H} o m(\cdot, \cdot) & : & \mathrm{D}^{\mathrm{b}}\left(\mathbf{k}_{M}\right)^{\mathrm{op}} \times \mathrm{D}^{\mathrm{b}}\left(\mathbf{k}_{M}\right) \rightarrow \mathrm{D}^{\mathrm{b}}\left(\mathbf{k}_{M}\right), \\
\bullet & \stackrel{\mathrm{L}}{\otimes}: & \mathrm{D}^{\mathrm{b}}\left(\mathbf{k}_{M}\right) \times \mathrm{D}^{\mathrm{b}}\left(\mathbf{k}_{M}\right) \rightarrow \mathrm{D}^{\mathrm{b}}\left(\mathbf{k}_{M}\right) .
\end{array}
$$

Hence, $\mathrm{D}^{\mathrm{b}}\left(\mathbf{k}_{M}\right)$ has a structure of commutative tensor category with $\mathbf{k}_{M}$ as unit object and R $\mathscr{H}$ om is the inner hom of this tensor category.

Now let $f: M \rightarrow N$ be a morphism of good topological spaces. One has the functors

$$
\begin{aligned}
& f^{-1}: \mathrm{D}^{\mathrm{b}}\left(\mathbf{k}_{N}\right) \rightarrow \mathrm{D}^{\mathrm{b}}\left(\mathbf{k}_{M}\right) \text { inverse image, } \\
& f^{!}: \mathrm{D}^{\mathrm{b}}\left(\mathbf{k}_{N}\right) \rightarrow \mathrm{D}^{\mathrm{b}}\left(\mathbf{k}_{M}\right) \text { extraordinary inverse image, } \\
& \mathrm{R} f_{*}: \mathrm{D}^{\mathrm{b}}\left(\mathbf{k}_{M}\right) \rightarrow \mathrm{D}^{\mathrm{b}}\left(\mathbf{k}_{N}\right) \text { direct image, } \\
& \mathrm{R} f_{!}: \mathrm{D}^{\mathrm{b}}\left(\mathbf{k}_{M}\right) \rightarrow \mathrm{D}^{\mathrm{b}}\left(\mathbf{k}_{N}\right) \text { proper direct image. }
\end{aligned}
$$

We get the pairs of adjoint functors $\left(f^{-1}, \mathrm{R} f_{*}\right)$ and $\left(\mathrm{R} f_{!}, f^{!}\right)$.

The operations associated with the functors $\otimes, \mathrm{R} \mathscr{H} o m, f^{-1}, f^{!}, \mathrm{R} f_{*}, \mathrm{R} f_{\text {! }}$ are called Grothendieck's six operations.

For two topological spaces $M$ and $N$, one defines the functor of external tensor product

$$
\bullet \bullet \cdot: \mathrm{D}^{\mathrm{b}}\left(\mathbf{k}_{M}\right) \times \mathrm{D}^{\mathrm{b}}\left(\mathbf{k}_{N}\right) \rightarrow \mathrm{D}^{\mathrm{b}}\left(\mathbf{k}_{M \times N}\right)
$$

by setting $F \otimes G:=q_{1}^{-1} F \otimes q_{2}^{-1} G$, where $q_{1}$ and $q_{2}$ are the projections from $M \times N$ to $M$ and $N$, respectively.

Denote by pt the topological space with a single element and by $a_{M}: M \rightarrow$ pt the unique morphism. One has the isomorphism

$$
\mathbf{k}_{M} \simeq a_{M}^{-1} \mathbf{k}_{\mathrm{pt}}, \quad \omega_{M} \simeq a_{M}^{!} \mathbf{k}_{\mathrm{pt}} .
$$

There are many important formulas relying the six operations. In particular we have the formulas below in which $F, F_{1}, F_{2} \in \mathrm{D}^{\mathrm{b}}\left(\mathbf{k}_{M}\right), G, G_{1}, G_{2} \in$ 
$\mathrm{D}^{\mathrm{b}}\left(\mathbf{k}_{N}\right)$ :

$$
\begin{aligned}
& \mathrm{R} \mathscr{H} o m\left(F \otimes F_{1}, F_{2}\right) \simeq \mathrm{R} \mathscr{H} o m\left(F, \operatorname{R} \mathscr{H} o m\left(F_{1}, F_{2}\right)\right), \\
& \mathrm{R} f_{*} \operatorname{R} \mathscr{H} o m\left(f^{-1} G, F\right) \simeq \mathrm{R} \mathscr{H} o m\left(G, \mathrm{R} f_{*} F\right), \\
& \mathrm{R} f_{!}\left(F \otimes f^{-1} G\right) \simeq F \otimes \mathrm{R} f_{!} G \quad(\text { projection formula }), \\
& f^{!} \operatorname{R} \mathscr{H} o m\left(G_{1}, G_{2}\right) \simeq \operatorname{R} \mathscr{H} o m\left(f^{-1} G_{1}, f^{!} G_{2}\right),
\end{aligned}
$$

and for a Cartesian square of good topological spaces,

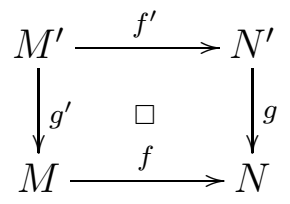

we have the base change formula!for sheaves

$$
g^{-1} \mathrm{R} f_{!} \simeq \mathrm{R} f_{!}^{\prime} g^{\prime-1} .
$$

In these Notes, we shall also encounter $\mathbb{R}$-constructible sheaves. References are made to [KS90, Ch. VIII]. Let $M$ be a real analytic manifold. On $M$ there is the family of subanalytic sets due to Hironaka and Gabrielov (see [BM 88, VD 98] for an exposition). This family is stable by all usual operations (finite intersection and locally finite union, complement, closure, interior) and contains the family of semi-analytic sets (those locally defined by analytic inequalities). If $f: M \rightarrow N$ is a morphism of real analytic manifolds, then the inverse image of a subanalytic set is subanalytic. If $Z$ is subanalytic in $M$ and $f$ is proper on the closure of $Z$, then $f(Z)$ is subanalytic in $N$.

A sheaf $F$ is $\mathbb{R}$-constructible if there exists a subanalytic stratification $M=\bigsqcup_{j \in J} M_{j}$ such that for each $j \in J$, the sheaf $\left.F\right|_{M_{j}}$ is locally constant of finite rank. One defines the category $\mathrm{D}_{\mathbb{R}-\mathrm{c}}^{\mathrm{b}}\left(\mathbf{k}_{M}\right)$ as the full subcategory of $\mathrm{D}^{\mathrm{b}}\left(\mathbf{k}_{M}\right)$ consisting of objects $F$ such that $H^{i}(F)$ is $\mathbb{R}$-constructible for all $i \in \mathbb{Z}$ and one proves that this category is triangulated.

The category $\mathrm{D}_{\mathbb{R}-\mathrm{c}}^{\mathrm{b}}\left(\mathbf{k}_{M}\right)$ is stable by the usual internal operations (tensor product, internal hom) and the duality functors in (1.1.1) induce antiequivalences on this category.

If $f: M \rightarrow N$ is a morphism of real analytic manifolds, then $f^{-1}$ and $f^{!}$send $\mathbb{R}$-constructible objects to $\mathbb{R}$-constructible objects. If $F \in \mathrm{D}_{\mathbb{R}-\mathrm{c}}^{\mathrm{b}}\left(\mathbf{k}_{M}\right)$ and $f$ is proper on $\operatorname{Supp}(F)$, then $\mathrm{R} f_{!} F \in \mathrm{D}_{\mathbb{R}-\mathrm{c}}^{\mathrm{b}}\left(\mathbf{k}_{N}\right)$. 


\subsection{D-modules}

References for D-module theory are made to [Ka03]. See also [Ka70, Bj93, HTT08].

Here, we shall briefly recall some basic constructions in the theory of D-modules that we shall use. Note that many classical functors that shall appear in this section will be extended to indsheaves in Section 3 and the subsequent sections.

In this subsection, the base field is the complex number field $\mathbb{C}$.

Let $\left(X, \mathscr{O}_{X}\right)$ be a complex manifold. We denote as usual by

- $d_{X}$ the complex dimension of $X$,

- $\Omega_{X}$ the invertible sheaf of differential forms of top degree,

- $\Omega_{X / Y}$ the invertible $\mathscr{O}_{X}$-module $\Omega_{X} \otimes_{f^{-1} \mathscr{O}_{Y}} f^{-1}\left(\Omega_{Y}^{\otimes-1}\right)$ for a morphism $f: X \rightarrow Y$ of complex manifolds,

- $\Theta_{X}$ the sheaf of holomorphic vector fields,

- $\mathscr{D}_{X}$ the sheaf of algebras of finite-order differential operators.

Denote by $\operatorname{Mod}\left(\mathscr{D}_{X}\right)$ the abelian category of left $\mathscr{D}_{X}$-modules and by $\operatorname{Mod}\left(\mathscr{D}_{X}^{\text {op }}\right)$ that of right $\mathscr{D}_{X}$-modules. There is an equivalence

$$
\mathrm{r}: \operatorname{Mod}\left(\mathscr{D}_{X}\right) \stackrel{\sim}{\longrightarrow} \operatorname{Mod}\left(\mathscr{D}_{X}^{\mathrm{op}}\right), \quad \mathscr{M} \mapsto \mathscr{M}^{\mathrm{r}}:=\Omega_{X} \otimes_{\mathscr{O}_{X}} \mathscr{M}
$$

By this equivalence, it is enough to study left $\mathscr{D}_{X}$-modules.

The ring $\mathscr{D}_{X}$ is coherent and one denotes by $\operatorname{Mod}_{\text {coh }}\left(\mathscr{D}_{X}\right)$ the thick abelian subcategory of $\operatorname{Mod}\left(\mathscr{D}_{X}\right)$ consisting of coherent modules.

To a coherent $\mathscr{D}_{X}$-module $\mathscr{M}$ one associates its characteristic variety $\operatorname{char}(\mathscr{M})$, a closed $\mathbb{C}^{\times}$-conic co-isotropic (one also says involutive) $\mathbb{C}$-analytic subset of the cotangent bundle $T^{*} X$. The involutivity property is a central theorem of the theory and is due to [SKK 73]. A purely algebraic proof was obtained later in [Ga81].

If $\operatorname{char}(\mathscr{M})$ is Lagrangian, $\mathscr{M}$ is called holonomic. It is immediately checked that the full subcategory $\operatorname{Mod}_{\text {hol }}\left(\mathscr{D}_{X}\right)$ of $\operatorname{Mod}_{\text {coh }}\left(\mathscr{D}_{X}\right)$ consisting of holonomic $\mathscr{D}$-modules is a thick abelian subcategory.

A $\mathscr{D}_{X}$-module $\mathscr{M}$ is quasi-good if, for any relatively compact open subset $U \subset X,\left.\mathscr{M}\right|_{U}$ is a sum of coherent $\left(\left.\mathscr{O}_{X}\right|_{U}\right)$-submodules. A $\mathscr{D}_{X}$-module $\mathscr{M}$ is 
good if it is quasi-good and coherent. The subcategories of $\operatorname{Mod}\left(\mathscr{D}_{X}\right)$ consisting of quasi-good (resp. good) $\mathscr{D}_{X}$-modules are abelian and thick. Therefore, one has the triangulated categories

- $\mathrm{D}_{\text {coh }}^{\mathrm{b}}\left(\mathscr{D}_{X}\right)=\left\{\mathscr{M} \in \mathrm{D}^{\mathrm{b}}\left(\mathscr{D}_{X}\right) ; H^{j}(\mathscr{M})\right.$ is coherent for all $\left.j \in \mathbb{Z}\right\}$,

- $\mathrm{D}_{\text {hol }}^{\mathrm{b}}\left(\mathscr{D}_{X}\right)=\left\{\mathscr{M} \in \mathrm{D}^{\mathrm{b}}\left(\mathscr{D}_{X}\right) ; H^{j}(\mathscr{M})\right.$ is holonomic for all $\left.j \in \mathbb{Z}\right\}$,

- $\mathrm{D}_{\text {q-good }}^{\mathrm{b}}\left(\mathscr{D}_{X}\right)=\left\{\mathscr{M} \in \mathrm{D}^{\mathrm{b}}\left(\mathscr{D}_{X}\right) ; H^{j}(\mathscr{M})\right.$ is quasi-good for all $\left.j \in \mathbb{Z}\right\}$,

- $\mathrm{D}_{\text {good }}^{\mathrm{b}}\left(\mathscr{D}_{X}\right)=\left\{\mathscr{M} \in \mathrm{D}^{\mathrm{b}}\left(\mathscr{D}_{X}\right) ; H^{j}(\mathscr{M})\right.$ is good for all $\left.j \in \mathbb{Z}\right\}$.

One may also consider the unbounded derived categories $\mathrm{D}\left(\mathscr{D}_{X}\right), \mathrm{D}^{-}\left(\mathscr{D}_{X}\right)$ and $\mathrm{D}^{+}\left(\mathscr{D}_{X}\right)$ and the full triangulated subcategories consisting of coherent, holonomic, quasi-good and good modules.

We have the functors

$$
\begin{aligned}
& \mathrm{R} \mathscr{H} o m_{\mathscr{D}_{X}}(\cdot, \cdot): \mathrm{D}^{\mathrm{b}}\left(\mathscr{D}_{X}\right)^{\mathrm{op}} \times \mathrm{D}^{\mathrm{b}}\left(\mathscr{D}_{X}\right) \rightarrow \mathrm{D}^{+}\left(\mathbb{C}_{X}\right), \\
& \text { - }{\stackrel{\mathrm{L}}{\mathscr{D}_{X}}} \cdot \quad: \quad \mathrm{D}^{\mathrm{b}}\left(\mathscr{D}_{X}^{\mathrm{op}}\right) \times \mathrm{D}^{\mathrm{b}}\left(\mathscr{D}_{X}\right) \rightarrow \mathrm{D}^{-}\left(\mathbb{C}_{X}\right) .
\end{aligned}
$$

We also have the functors

$$
\begin{array}{lll}
\cdot \stackrel{\mathrm{D}}{\otimes} \cdot: & \mathrm{D}^{-}\left(\mathscr{D}_{X}\right) \times \mathrm{D}^{-}\left(\mathscr{D}_{X}\right) \rightarrow \mathrm{D}^{-}\left(\mathscr{D}_{X}\right), \\
\cdot \stackrel{\mathrm{D}}{\otimes} \cdot: & \mathrm{D}^{-}\left(\mathscr{D}_{X}^{\mathrm{op}}\right) \times \mathrm{D}^{-}\left(\mathscr{D}_{X}\right) \rightarrow \mathrm{D}^{-}\left(\mathscr{D}_{X}^{\mathrm{op}}\right),
\end{array}
$$

constructed as follows. The $\left(\mathscr{D}_{X}, \mathscr{D}_{X} \otimes \mathscr{D}_{X}\right)$-bimodule structure on $\mathscr{D}_{X} \otimes_{\mathscr{O}_{X}}$ $\mathscr{D}_{X}$ gives

$$
\mathscr{M} \otimes_{\mathscr{O}_{X}} \mathscr{N} \simeq\left(\mathscr{D}_{X} \otimes_{\mathscr{O}_{X}} \mathscr{D}_{X}\right) \otimes_{\mathscr{D}_{X} \otimes \mathscr{D}_{X}}(\mathscr{M} \otimes \mathscr{N})
$$

the structure of a $\mathscr{D}_{X}$-module for $\mathscr{M}$ and $\mathscr{N}$ two $\mathscr{D}_{X}$-modules, and similarly for $\mathscr{N}$ a right $\mathscr{D}_{X}$-module.

There are similar constructions with right $\mathscr{D}_{X}$-modules.

One defines the duality functor for D-modules by setting

$$
\begin{aligned}
& \mathbb{D}_{X} \mathscr{M}=\mathrm{R} \mathscr{H} o m_{\mathscr{D}_{X}}\left(\mathscr{M}, \mathscr{D}_{X} \otimes_{\mathscr{O}_{X}} \Omega_{X}^{\otimes-1}\right)\left[d_{X}\right] \in \mathrm{D}^{\mathrm{b}}\left(\mathscr{D}_{X}\right) \quad \text { for } \mathscr{M} \in \mathrm{D}^{\mathrm{b}}\left(\mathscr{D}_{X}\right), \\
& \mathbb{D}_{X} \mathscr{N}=\mathrm{R} \mathscr{H} m_{\mathscr{D}_{X}^{\text {op }}}\left(\mathscr{N}, \Omega_{X} \otimes_{\mathscr{O}_{X}} \mathscr{D}_{X}\right)\left[d_{X}\right] \in \mathrm{D}^{\mathrm{b}}\left(\mathscr{D}_{X}^{\mathrm{op}}\right) \quad \text { for } \mathscr{N} \in \mathrm{D}^{\mathrm{b}}\left(\mathscr{D}_{X}^{\mathrm{op}}\right) .
\end{aligned}
$$




\section{$1.2 \quad$ D-modules}

Let $X$ and $Y$ be two complex manifolds. One defines the functor of external tensor product for D-modules

$$
\stackrel{\mathrm{D}}{\nabla} \cdot: \mathrm{D}^{\mathrm{b}}\left(\mathscr{D}_{X}\right) \times \mathrm{D}^{\mathrm{b}}\left(\mathscr{D}_{Y}\right) \rightarrow \mathrm{D}^{\mathrm{b}}\left(\mathscr{D}_{X \times Y}\right)
$$

by setting $\mathscr{M} \stackrel{\mathrm{D}}{\otimes} \mathscr{N}=\mathscr{D}_{X \times Y} \otimes_{\mathscr{D}_{X} \otimes \mathscr{D}_{Y}}(\mathscr{M} \otimes \mathscr{N})$.

Now, let $f: X \rightarrow Y$ be a morphism of complex manifolds. The transfer bimodule $\mathscr{D}_{X \rightarrow Y}$ is a $\left(\mathscr{D}_{X}, f^{-1} \mathscr{D}_{Y}\right)$ bimodule defined as follows. As an $\left(\mathscr{O}_{X}, f^{-1} \mathscr{D}_{Y}\right)$-bimodule, $\mathscr{D}_{X \rightarrow Y}=\mathscr{O}_{X} \otimes_{f^{-1} \mathscr{O}_{Y}} f^{-1} \mathscr{D}_{Y}$. The left $\mathscr{D}_{X}$-module structure of $\mathscr{D}_{X \rightarrow Y}$ is deduced from the action of $\Theta_{X}$. For $v \in \Theta_{X}$, denoting by $\sum_{i} a_{i} \otimes w_{i}$ its image in $\mathscr{O}_{X} \otimes_{f^{-1} \mathscr{O}_{Y}} f^{-1} \Theta_{Y}$, the action of $v$ on $\mathscr{D}_{X \rightarrow Y}$ is given by

$$
v(a \otimes P)=v(a) \otimes P+\sum_{i} a a_{i} \otimes w_{i} P .
$$

One also uses the opposite transfer bimodule $\mathscr{D}_{Y \leftarrow X}=f^{-1} \mathscr{D}_{Y} \otimes_{f^{-1} \mathscr{O}_{Y}} \Omega_{X / Y}$, an $\left(f^{-1} \mathscr{D}_{Y}, \mathscr{D}_{X}\right)$-bimodule.

Note that for another morphism of complex manifolds $g: Y \rightarrow Z$, one has the natural isomorphisms

$$
\begin{aligned}
& \mathscr{D}_{X \rightarrow Y} \stackrel{\mathrm{L}}{\otimes}_{f^{-1} \mathscr{D}_{Y}} f^{-1} \mathscr{D}_{Y \rightarrow Z} \simeq \mathscr{D}_{X \rightarrow Z}, \\
& f^{-1} \mathscr{D}_{Z \leftarrow Y} \stackrel{\mathrm{L}}{\otimes_{f^{-1}} \mathscr{D}_{Y}} \mathscr{D}_{Y \leftarrow X} \simeq \mathscr{D}_{Z \leftarrow X} .
\end{aligned}
$$

One can now define the external operations on D-modules by setting:

$$
\begin{aligned}
& \mathrm{D}^{*} \mathscr{N}:=\mathscr{D}_{X} \rightarrow Y \stackrel{\mathrm{L}}{\otimes}_{f^{-1} \mathscr{D}_{Y}} f^{-1} \mathscr{N}, \text { for } \mathscr{N} \in \mathrm{D}^{\mathrm{b}}\left(\mathscr{D}_{Y}\right), \\
& \mathrm{D} f_{!} \mathscr{M}:=\mathrm{R} f_{!}\left(\mathscr{M}^{\mathrm{L}}{ }_{\mathscr{D}_{X}} \mathscr{D}_{X} \rightarrow Y\right) \text { for } \mathscr{M} \in \mathrm{D}^{\mathrm{b}}\left(\mathscr{D}_{X}^{\text {op }}\right),
\end{aligned}
$$

and one defines $\mathrm{D} f_{*} \mathscr{M}$ by replacing $\mathrm{R} f_{!}$with $\mathrm{R} f_{*}$ in the above formula. By using the opposite transfer bimodule $\mathscr{D}_{Y \leftarrow X}$ one defines similarly the inverse image of a right $\mathscr{D}_{Y}$-module or the direct image of a left $\mathscr{D}_{X}$-module.

One calls respectively $\mathrm{D} f^{*}, \mathrm{D} f_{*}$ and $\mathrm{D} f_{\text {! }}$ the inverse image, direct image and proper direct image functors in the category of D-modules.

Note that

$$
\mathrm{D} f^{*} \mathscr{O}_{Y} \simeq \mathscr{O}_{X}, \quad \mathrm{D} f^{*} \Omega_{Y} \simeq \Omega_{X}
$$


Also note that the properties of being quasi-good are stable by inverse image and tensor product, as well as by direct image by maps proper on the support of the module. The property of being good is stable by duality.

Let $f: X \rightarrow Y$ be a morphism of complex manifolds. One associates the maps

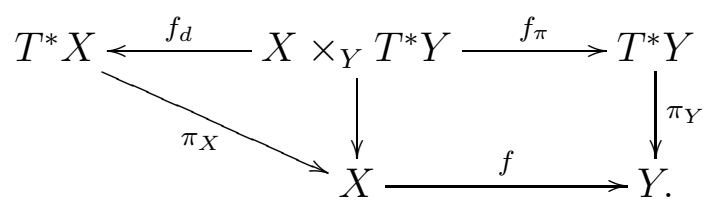

One says that $f$ is non-characteristic for $\mathscr{N} \in \mathrm{D}_{\text {coh }}^{\mathrm{b}}\left(\mathscr{D}_{Y}\right)$ if the map $f_{d}$ is proper (hence, finite) on $f_{\pi}^{-1}(\operatorname{char}(\mathscr{N}))$.

The classical de Rham and solution functors are defined by

$$
\begin{aligned}
\mathcal{D} \mathcal{R}_{X}: \mathrm{D}^{\mathrm{b}}\left(\mathscr{D}_{X}\right) \rightarrow \mathrm{D}^{\mathrm{b}}\left(\mathbb{C}_{X}\right), & \mathscr{M} \mapsto \Omega_{X} \stackrel{\mathrm{L}}{\mathscr{\otimes}_{\mathscr{D}_{X}} \mathscr{M},} \\
\mathcal{S}_{l_{X}}: \mathrm{D}^{\mathrm{b}}\left(\mathscr{D}_{X}\right)^{\mathrm{op}} \rightarrow \mathrm{D}^{\mathrm{b}}\left(\mathbb{C}_{X}\right), & \mathscr{M} \mapsto \mathrm{R} \mathscr{H} \mathrm{om}_{\mathscr{D}_{X}}\left(\mathscr{M}, \mathscr{O}_{X}\right) .
\end{aligned}
$$

For $\mathscr{M} \in \mathrm{D}_{\text {coh }}^{\mathrm{b}}\left(\mathscr{D}_{X}\right)$, one has

$$
\operatorname{Sol}_{X}(\mathscr{M}) \simeq \mathcal{D} \mathcal{R}_{X}\left(\mathbb{D}_{X} \mathscr{M}\right)\left[-d_{X}\right] .
$$

Theorem 1.2.1 (Projection formulas [Ka03, Theorems 4.2.8, 4.40]). Let $f: X \rightarrow Y$ be a morphism of complex manifolds. Let $\mathscr{M} \in \mathrm{D}^{\mathrm{b}}\left(\mathscr{D}_{X}\right)$ and $\mathscr{L} \in \mathrm{D}^{\mathrm{b}}\left(\mathscr{D}_{Y}^{\mathrm{op}}\right)$. There are natural isomorphisms:

$$
\begin{aligned}
\mathrm{D} f_{!}\left(\mathrm{D} f^{*} \mathscr{L} \stackrel{\mathrm{D}}{\otimes} \mathscr{M}\right) & \simeq \mathscr{L} \stackrel{\mathrm{D}}{\otimes} \mathrm{D} f_{!} \mathscr{M}, \\
\mathrm{R}_{!}\left(\mathrm{D} f^{*} \mathscr{L} \stackrel{\mathrm{L}}{\otimes}_{\mathscr{D}_{X}} \mathscr{M}\right) & \simeq \mathscr{L} \stackrel{\mathrm{L}}{\otimes}_{\mathscr{D}_{Y}} \mathrm{D} f_{!} \mathscr{M} .
\end{aligned}
$$

In particular, there is an isomorphism (commutation of the de Rham functor and direct images)

$$
\operatorname{Rf}_{!}\left(\mathcal{D R} \mathcal{R}_{X}(\mathscr{M})\right) \simeq \mathcal{D} \mathcal{R}_{Y}\left(\mathrm{D} f_{!} \mathscr{M}\right) .
$$

Theorem 1.2.2 (Commutativity with duality [Ka03, Sc 86]). Let $f: X \rightarrow Y$ be a morphism of complex manifolds.

(i) Let $\mathscr{M} \in \mathrm{D}_{\text {good }}^{\mathrm{b}}\left(\mathscr{D}_{X}\right)$ and assume that $f$ is proper on $\operatorname{Supp}(\mathscr{M})$. Then $\mathrm{D} f_{!} \mathscr{M} \in \mathrm{D}_{\text {good }}^{\mathrm{b}}\left(\mathscr{D}_{Y}\right)$ and $\mathbb{D}_{Y}\left(\mathrm{D} f_{!} \mathscr{M}\right) \simeq \mathrm{D} f_{!} \mathbb{D}_{X} \mathscr{M}$. 
(ii) Let $\mathscr{N} \in \mathrm{D}_{\text {q-good }}^{\mathrm{b}}\left(\mathscr{D}_{Y}\right)$. Then $\mathrm{D} f^{*} \mathscr{N} \in \mathrm{D}_{\text {q-good }}^{\mathrm{b}}\left(\mathscr{D}_{X}\right)$. Moreover, if $\mathscr{N} \in$ $\mathrm{D}_{\text {coh }}^{\mathrm{b}}\left(\mathscr{D}_{Y}\right)$ and $f$ is non-characteristic for $\mathscr{N}$, then $\mathrm{D} f^{*} \mathscr{N} \in \mathrm{D}_{\mathrm{coh}}^{\mathrm{b}}\left(\mathscr{D}_{X}\right)$ and $\mathbb{D}_{X}\left(\mathrm{D} f^{*} \mathscr{N}\right) \simeq \mathrm{D} f^{*} \mathbb{D}_{Y} \mathscr{N}$.

Corollary 1.2.3. Let $f: X \rightarrow Y$ be a morphism of complex manifolds.

(i) Let $\mathscr{M} \in \mathrm{D}_{\text {good }}^{\mathrm{b}}\left(\mathscr{D}_{X}\right)$ and assume that $f$ is proper on $\operatorname{Supp}(\mathscr{M})$. Then we have the isomorphism for $\mathscr{N} \in \mathrm{D}\left(\mathscr{D}_{Y}\right)$ :

(1.2.6) $\mathrm{R} f_{*} \mathrm{R}_{\mathscr{H} o m_{\mathscr{D}_{X}}}\left(\mathscr{M}, \mathrm{D} f^{*} \mathscr{N}\right)\left[d_{X}\right] \simeq \mathrm{R} \mathscr{H} o m_{\mathscr{D}_{Y}}\left(\mathrm{D} f_{*} \mathscr{M}, \mathscr{N}\right)\left[d_{Y}\right]$.

In particular, with the same hypotheses, we have the isomorphism (commutation of the Sol functor and direct images)

$$
\mathrm{R} f_{*} \mathrm{R} \mathscr{H}_{o m_{\mathscr{D}_{X}}}\left(\mathscr{M}, \mathscr{O}_{X}\right)\left[d_{X}\right] \simeq \mathrm{R} \mathscr{H}_{o m_{\mathscr{D}_{Y}}}\left(\mathrm{D} f_{*} \mathscr{M}, \mathscr{O}_{Y}\right)\left[d_{Y}\right]
$$

(ii) Let $\mathscr{N} \in \mathrm{D}_{\text {coh }}^{\mathrm{b}}\left(\mathscr{D}_{Y}\right)$ and assume that $f$ is non-characteristic for $\mathscr{N}$. Then we have the isomorphism for $\mathscr{M} \in \mathrm{D}\left(\mathscr{D}_{X}\right)$ :

$$
\mathrm{R} f_{*} \mathrm{R} \mathscr{H} o m_{\mathscr{D}_{X}}\left(\mathrm{D} f^{*} \mathscr{N}, \mathscr{M}\right)\left[d_{X}\right] \simeq \mathrm{R} \mathscr{H} o m_{\mathscr{D}_{Y}}\left(\mathscr{N}, \mathrm{D} f_{*} \mathscr{M}\right)\left[d_{Y}\right]
$$

A transversal Cartesian diagram is a commutative diagram

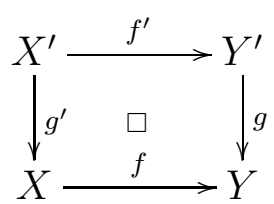

with $X^{\prime} \simeq X \times_{Y} Y^{\prime}$ and such that the map of tangent spaces

$$
T_{g^{\prime}(x)} X \oplus T_{f^{\prime}(x)} Y^{\prime} \rightarrow T_{f\left(g^{\prime}(x)\right)} Y
$$

is surjective for any $x \in X^{\prime}$.

Proposition 1.2.4 (Base change formula). Consider the transversal Cartesian diagram (1.2.9). Then, for any $\mathscr{M} \in \mathrm{D}_{\text {good }}^{\mathrm{b}}\left(\mathscr{D}_{X}\right)$ such that $\operatorname{Supp}(\mathscr{M})$ is proper over $Y$, we have the isomorphism

$$
\mathrm{D} g^{*} \mathrm{D} f_{*} \mathscr{M} \simeq \mathrm{D} f^{\prime}{ }_{*} \mathrm{D} g^{\prime *} \mathscr{M}
$$




\section{Indsheaves}

\subsection{Ind-objects}

References are made to [SGA4] or to [KS06] for an exposition on ind-objects.

Let $\mathscr{C}$ be a category (in a given universe). One denotes by $\mathscr{C}^{\wedge}$ the big category of functors from $\mathscr{C}^{\text {op }}$ to Set. By the fully faithful functor $h^{\wedge}: \mathscr{C} \rightarrow$ $\mathscr{C}^{\wedge}$, we regard $\mathscr{C}$ as a full subcategory of $\mathscr{C}^{\wedge}$.

An ind-object in $\mathscr{C}$ is an object $A \in \mathscr{C}^{\wedge}$ which is isomorphic to "lim" $\underset{i \in I}{\longrightarrow} X_{i}$ where $X_{i} \in \mathscr{C}$ and $I$ filtrant and small. Here, "lim" is the inductive limit in $\mathscr{C}^{\wedge}$. One denotes by $\operatorname{Ind}(\mathscr{C})$ the full subcategory of $\mathscr{C}^{\wedge}$ consisting of ind-objects.

Theorem 2.1.1. Let $\mathscr{C}$ be an abelian category.

(i) The category $\operatorname{Ind}(\mathscr{C})$ is abelian.

(ii) The natural functors $\iota: \mathscr{C} \rightarrow \operatorname{Ind}(\mathscr{C})$ and $\operatorname{Ind}(\mathscr{C}) \rightarrow \mathscr{C}^{\wedge}$ are fully faithful.

(iii) The category $\operatorname{Ind}(\mathscr{C})$ admits exact small filtrant inductive limits, also denoted by "lim" and the functor $\operatorname{Ind}(\mathscr{C}) \rightarrow \mathscr{C}^{\wedge}$ commutes with such limits.

(iv) Assume that $\mathscr{C}$ admits small projective limits. Then the category $\operatorname{Ind}(\mathscr{C})$ admits small projective limits, and the functor $\mathscr{C} \rightarrow \operatorname{Ind}(\mathscr{C})$ commutes with such limits.

(v) Assume that $\mathscr{C}$ admits small inductive limits, denoted by $\lim _{\mathrm{T}}$. Then the functor $\iota$ admits a left adjoint $\alpha$. For $X=\underset{i}{\underset{i}{\longrightarrow}} X_{i}$ with $X_{i} \in \mathscr{C}$ and $I$ small and filtrant, $\alpha(X) \simeq \underset{i}{\lim } X_{i}$.

Note that for $X=\underset{i}{\stackrel{\lim }{\longrightarrow}} X_{i}$ and $Y=\underset{j}{\stackrel{\lim }{\longrightarrow}} Y_{j} \in \operatorname{Ind}(\mathscr{C})$ with $X_{i}, Y_{j} \in \mathscr{C}$,

$$
\operatorname{Hom}_{\operatorname{Ind}(\mathscr{C})}(X, Y) \simeq \lim _{i} \underset{j}{\lim _{j}} \operatorname{Hom}_{\mathscr{C}}\left(X_{i}, Y_{j}\right)
$$


Example 2.1.2. Let $\mathbf{k}$ be a field. Denote by $\operatorname{Mod}(\mathbf{k})$ the category of $\mathbf{k}$-vector spaces and by $\operatorname{Mod}^{f}(\mathbf{k})$ its full subcategory consisting of finite-dimensional vector spaces. Denote for short by $\mathrm{I}(\mathbf{k})$ the category of ind-objects in $\operatorname{Mod}(\mathbf{k})$. The functor $\alpha: \mathrm{I}(\mathbf{k}) \rightarrow \operatorname{Mod}(\mathbf{k})$ admits a left adjoint $\beta: \operatorname{Mod}(\mathbf{k}) \rightarrow \mathrm{I}(\mathbf{k})$ defined as follows. For $V \in \operatorname{Mod}(\mathbf{k})$, set $\beta(V)=$ "lim" $W$, where $W$ ranges over the family of finite-dimensional vector subspaces of $V$. In other words, $\beta(V)$ is the functor

$$
\begin{aligned}
& \operatorname{Mod}(\mathbf{k})^{\mathrm{op}} \rightarrow \operatorname{Mod}(\mathbb{Z}), \\
& M \mapsto \underset{W \subset V}{\lim _{W C V}} \operatorname{Hom}_{k}(M, W), \quad W \text { finite-dimensional. }
\end{aligned}
$$

Note that $\beta(V)(M) \simeq \operatorname{Hom}_{\mathbf{k}}(M, \mathbf{k}) \otimes V$.

If $V$ is infinite-dimensional, $\beta(V)$ is not representable in $\operatorname{Mod}(\mathbf{k})$. Moreover, $\operatorname{Hom}_{\mathrm{I}(\mathbf{k})}(\mathbf{k}, V / \beta(V)) \simeq 0$.

Now, denote by $\mathrm{I}^{f}(\mathbf{k})$ the category of ind-objects in $\operatorname{Mod}^{f}(\mathbf{k})$. There is an equivalence of categories

$$
\alpha: \mathrm{I}^{f}(\mathbf{k}) \stackrel{\sim}{\longrightarrow} \operatorname{Mod}(\mathbf{k}), \quad \underset{i}{\stackrel{\lim }{\longrightarrow}} V_{i} \mapsto \underset{i}{\lim } V_{i} .
$$

We get the non commutative diagram of categories

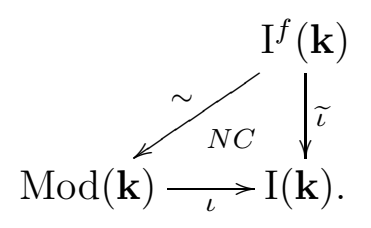

Moreover, the functor $\widetilde{\iota}$ commutes with small inductive limits but the functor $\iota$ does not.

It is proved in [KS06, Prop. 15.1.2] that the category I(k) does not have enough injectives.

Definition 2.1.3. An object $A \in \operatorname{Ind}(\mathscr{C})$ is quasi-injective if the functor $\operatorname{Hom}_{\operatorname{Ind}(\mathscr{C})}(\bullet, A)$ is exact on the category $\mathscr{C}$.

It is proved in loc. cit. that if $\mathscr{C}$ has enough injectives, then $\operatorname{Ind}(\mathscr{C})$ has enough quasi-injectives. 


\subsection{Indsheaves}

Let $M$ be a good topological space and let $\mathbf{k}$ be a field as in subsection 1.1.

One denotes by $\operatorname{Mod}^{\mathrm{c}}\left(\mathbf{k}_{M}\right)$ the full subcategory of $\operatorname{Mod}\left(\mathbf{k}_{M}\right)$ consisting of sheaves with compact support. We set for short:

$$
\mathrm{I}\left(\mathbf{k}_{M}\right):=\operatorname{Ind}\left(\operatorname{Mod}^{\mathrm{c}}\left(\mathbf{k}_{M}\right)\right)
$$

and calls an object of this category an indsheaf on $M$.

When there is no risk of confusion, we shall simply write $\mathbf{I k}_{M}$ instead of $\mathrm{I}\left(\mathbf{k}_{M}\right)$.

Theorem 2.2.1. The prestack $\mathcal{I}\left(\mathbf{k}_{M}\right): U \mapsto \mathrm{I}\left(\mathbf{k}_{U}\right), U$ open in $M$, is a stack.

For $F=\underset{i}{\stackrel{\lim }{\rightarrow}} F_{i} \in \mathrm{I}\left(\mathbf{k}_{M}\right)$ and $G=\underset{j}{\stackrel{\lim }{\rightarrow}} G_{j} \in \mathrm{I}\left(k_{M}\right)$ with $F_{i}, G_{j} \in$ $\operatorname{Mod}^{\mathrm{c}}\left(\mathbf{k}_{M}\right)$, we set:

$$
\begin{aligned}
& F \otimes G=\underset{i, j}{\stackrel{\lim }{\longrightarrow}}\left(F_{i} \otimes G_{j}\right), \\
& \mathscr{I h o m}(F, G)=\underset{\leftarrow}{\lim } \underset{i}{\stackrel{\lim }{\longrightarrow}} \mathscr{H} \text { om }\left(F_{i}, G_{j}\right) \text {. }
\end{aligned}
$$

Note that for $F \in \operatorname{Mod}\left(\mathbf{k}_{M}\right)$ and $\left\{G_{j}\right\}_{j \in J}$ a small filtrant inductive system in $\mathrm{I}\left(k_{M}\right)$, we have

$$
\mathscr{I h o m}\left(F, \underset{\vec{j}}{\stackrel{\lim }{\longrightarrow}} G_{j}\right) \simeq \underset{\vec{j}}{\stackrel{\lim }{\longrightarrow}} \mathscr{I h o m}\left(F, G_{j}\right) .
$$

Lemma 2.2.2. The category $\mathrm{I}\left(\mathbf{k}_{M}\right)$ is a tensor category with $\otimes$ as a tensor product and $\mathbf{k}_{M}$ as a unit object.

Note that $\mathscr{I h o m}$ is the inner hom of the tensor category $\mathrm{I}\left(\mathbf{k}_{M}\right)$, i.e., we have

$$
\operatorname{Hom}_{\mathrm{I}\left(\mathbf{k}_{M}\right)}\left(K_{1} \otimes K_{2}, K_{3}\right) \simeq \operatorname{Hom}_{\mathrm{I}\left(\mathbf{k}_{M}\right)}\left(K_{1}, \mathscr{I h o m}\left(K_{2}, K_{3}\right)\right) .
$$

We have two pairs $\left(\alpha_{M}, \iota_{M}\right)$ and $\left(\beta_{M}, \alpha_{M}\right)$ of adjoint functors

$$
\operatorname{Mod}\left(\mathbf{k}_{M}\right) \underset{\alpha_{M}}{\stackrel{\alpha_{M}}{\beta_{M}}} \mathrm{I}\left(\mathbf{k}_{M}\right) .
$$


The functor $\iota_{M}$ is given by

$$
\iota_{M} F=\underset{U \subset \mathrm{CC} M}{\stackrel{\text { lim" }}{\longrightarrow}} F_{U}, U \text { open relatively compact in } M .
$$

The functor $\alpha_{M}$ is defined by

$$
\alpha_{M}: \underset{i \in I}{\lim } F_{i} \mapsto \underset{i \in I}{\lim _{i \in I}} F_{i} \quad(I \text { small and filtrant }) .
$$

For $F \in \operatorname{Mod}\left(\mathbf{k}_{M}\right), \beta_{M}(F)$ is the functor

$$
\beta_{M}(F): G \mapsto \Gamma\left(M ; H^{0}\left(\mathrm{D}_{M}^{\prime} G\right) \otimes F\right), \quad\left(G \in \operatorname{Mod}^{\mathrm{c}}\left(\mathbf{k}_{M}\right)\right) .
$$

(This last formula is no more true if $\mathbf{k}$ is not a field.)

- $\iota_{M}$ is exact, fully faithful, and commutes with $\lim _{\leftarrow}$,

- $\alpha_{M}$ is exact and commutes with $\underset{\longleftarrow}{\lim }$ and $\stackrel{\lim }{\longrightarrow}$,

- $\beta_{M}$ is exact, fully faithful and commutes with $\stackrel{\lim }{\longrightarrow}$,

- $\alpha_{M}$ is left adjoint to $\iota_{M}$,

- $\alpha_{M}$ is right adjoint to $\beta_{M}$,

- $\alpha_{M} \circ \iota_{M} \simeq \operatorname{id}_{\operatorname{Mod}\left(\mathbf{k}_{M}\right)}$ and $\alpha_{M} \circ \beta_{M} \simeq \operatorname{id}_{\operatorname{Mod}\left(\mathbf{k}_{M}\right)}$.

Denote as usual by

$$
\mathscr{H}^{\circ} m_{\mathrm{Ik}_{M}}: \mathrm{I}\left(\mathbf{k}_{M}\right)^{\mathrm{op}} \times \mathrm{I}\left(\mathbf{k}_{M}\right) \rightarrow \operatorname{Mod}\left(\mathbf{k}_{M}\right)
$$

the hom functor of the stack $\mathcal{I}\left(\mathbf{k}_{M}\right)$. Then

$$
\mathscr{H} \mathrm{Im}_{\mathrm{Ik}_{M}} \simeq \alpha_{M} \circ \mathscr{I h o m}
$$

and

$$
\operatorname{Hom}_{\mathrm{I}\left(\mathbf{k}_{M}\right)}\left(K_{1}, K_{2}\right) \simeq \Gamma\left(M ; \mathscr{H} o m_{\mathrm{Ik}_{M}}\left(K_{1}, K_{2}\right)\right) \quad \text { for } K_{1}, K_{2} \in \mathrm{I}\left(\mathbf{k}_{M}\right) .
$$

Notation 2.2.3. As far as there is no risk of confusion, we shall not write the functor $\iota_{M}$. Hence, we identify a sheaf $F$ on $M$ and its image by $\iota_{M}$. 
Example 2.2.4. Let $U \subset M$ be an open subset, $S \subset M$ a closed subset. Then

$$
\begin{aligned}
& \beta_{M}\left(\mathbf{k}_{U}\right) \simeq \underset{V}{\stackrel{\lim }{V}} \mathbf{k}_{V}, V \text { open }, V \subset \subset U, \\
& \beta_{M}\left(\mathbf{k}_{S}\right) \simeq \underset{V}{\stackrel{\lim }{V}} \mathbf{k}_{\bar{V}}, V \text { open }, S \subset V .
\end{aligned}
$$

Let $a \in M$ and consider the skyscraper sheaf $\mathbf{k}_{\{a\}}$. Then $\beta_{M}\left(\mathbf{k}_{\{a\}}\right) \rightarrow \mathbf{k}_{\{a\}}$ is an epimorphism in $\mathrm{I}\left(\mathbf{k}_{M}\right)$ and defining $N_{a}$ by the exact sequence:

$$
0 \rightarrow N_{a} \rightarrow \beta_{M}\left(\mathbf{k}_{\{a\}}\right) \rightarrow \mathbf{k}_{\{a\}} \rightarrow 0,
$$

we get that $\operatorname{Hom}_{\operatorname{Ik}_{M}}\left(\mathbf{k}_{U}, N_{a}\right) \simeq 0$ for all open neighborhood $U$ of $a$.

Let $f: M \rightarrow N$ be a continuous map. Let $G=\underset{i}{\stackrel{\text { "lim" }}{\longrightarrow}} G_{i} \in \mathrm{I}\left(k_{N}\right)$ with $G_{i} \in \operatorname{Mod}^{\mathrm{c}}\left(\mathbf{k}_{N}\right)$. One defines $f^{-1} G \in \mathrm{I}\left(\mathbf{k}_{M}\right)$ by the formula

$$
f^{-1} G=\underset{i}{\stackrel{\lim }{\longrightarrow}} f^{-1} G_{i} .
$$

Let $F=\underset{i}{\stackrel{\text { lim" }}{\longrightarrow}} F_{i} \in \mathrm{I}\left(\mathbf{k}_{M}\right)$ with $F_{i} \in \operatorname{Mod}^{\mathrm{c}}\left(k_{M}\right)$. One defines $f_{*} F \in \mathrm{I}\left(\mathbf{k}_{N}\right)$ by the formula:

$$
f_{*}\left(\underset{i}{\stackrel{\lim }{\longrightarrow}} F_{i}\right)=\underset{K}{\lim } \underset{i}{\stackrel{\lim }{\longrightarrow}} f_{*}\left(F_{i K}\right)(K \text { compact in } M) .
$$

The two functors $f_{*}$ and $f^{-1}$ commute with both the functors $\iota$ and $\alpha$ and that is the reason why we keep the same notations as for usual sheaves. Recall that for a usual sheaf $F$, its proper direct image is defined by

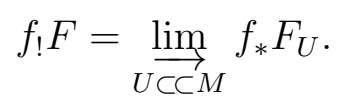
Hence, one defines the proper direct image of $F=\underset{i}{\stackrel{\lim }{\vec{c}}} F_{i} \in \mathrm{I}\left(\mathbf{k}_{M}\right)$ with
$F_{i} \in \operatorname{Mod}^{\mathrm{c}}\left(k_{M}\right)$ by

$$
f_{! !}\left(\underset{i}{\stackrel{\lim }{\longrightarrow}} F_{i}\right)=\underset{i}{\stackrel{\lim }{\longrightarrow}} f_{*}\left(F_{i}\right) .
$$

However, $f_{! !} \circ \iota_{M} \neq \iota_{N} \circ f_{!}$in general. That is why we have used a different notation. 


\subsection{Ring action}

The category $\mathrm{I}\left(\mathbf{k}_{M}\right)$ does not have enough injectives, even for $M=\mathrm{pt}$ as already mentioned. In particular, it is not a Grothendieck category. One can however construct the derived functors and the six operations for indsheaves. The functor $f^{-1}$ has a right adjoint $\mathrm{R} f_{*}$. The functor $\mathrm{R} f_{\text {!! }}$ admits a right adjoint, denoted by $f^{!}$. Hence we have functors

$$
\begin{aligned}
& \iota_{M} \quad: \quad \mathrm{D}^{\mathrm{b}}\left(\mathbf{k}_{M}\right) \rightarrow \mathrm{D}^{\mathrm{b}}\left(\mathrm{I}_{M}\right), \\
& \alpha_{M} \quad: \quad \mathrm{D}^{\mathrm{b}}\left(\mathrm{I}_{M}\right) \rightarrow \mathrm{D}^{\mathrm{b}}\left(\mathbf{k}_{M}\right), \\
& \beta_{M}: \quad \mathrm{D}^{\mathrm{b}}\left(\mathbf{k}_{M}\right) \rightarrow \mathrm{D}^{\mathrm{b}}\left(\mathrm{I}_{M}\right), \\
& \otimes: \quad \mathrm{D}^{\mathrm{b}}\left(\mathrm{Ik}_{M}\right) \times \mathrm{D}^{\mathrm{b}}\left(\mathrm{Ik}_{M}\right) \rightarrow \mathrm{D}^{\mathrm{b}}\left(\mathrm{Ik}_{M}\right), \\
& \mathrm{R} \mathscr{\text { Ihom }}: \mathrm{D}^{\mathrm{b}}\left(\mathrm{Ik}_{M}\right)^{\mathrm{op}} \times \mathrm{D}^{\mathrm{b}}\left(\mathrm{Ik}_{M}\right) \rightarrow \mathrm{D}^{+}\left(\mathrm{Ik}_{M}\right), \\
& \mathrm{R} \mathscr{H}_{0 m_{\mathrm{Ik}_{M}}}: \mathrm{D}^{\mathrm{b}}\left(\mathrm{Ik}_{M}\right)^{\mathrm{op}} \times \mathrm{D}^{\mathrm{b}}\left(\mathrm{Ik}_{M}\right) \rightarrow \mathrm{D}^{+}\left(\mathbf{k}_{M}\right), \\
& \mathrm{R} f_{*}: \mathrm{D}^{\mathrm{b}}\left(\mathrm{Ik}_{M}\right) \rightarrow \mathrm{D}^{\mathrm{b}}\left(\mathrm{Ik}_{N}\right) \\
& f^{-1}: \mathrm{D}^{\mathrm{b}}\left(\mathrm{Ik}_{N}\right) \rightarrow \mathrm{D}^{\mathrm{b}}\left(\mathrm{Ik}_{M}\right), \\
& \mathrm{R} f_{! !}: \quad \mathrm{D}^{\mathrm{b}}\left(\mathrm{Ik}_{M}\right) \rightarrow \mathrm{D}^{\mathrm{b}}\left(\mathrm{Ik}_{N}\right), \\
& f^{!}: \quad \mathrm{D}^{\mathrm{b}}\left(\mathrm{Ik}_{N}\right) \rightarrow \mathrm{D}^{\mathrm{b}}\left(\mathrm{Ik}_{M}\right) \text {. }
\end{aligned}
$$

We may summarize the commutativity of the various functors we have introduced in the table below. Here, "o" means that the functors commute, and " $\times$ " they do not. Moreover, $\underset{\longrightarrow}{\longrightarrow}$ are taken over small filtrant categories.

\begin{tabular}{c|c|c|c|c|c|c|c|} 
& $\otimes$ & $f^{-1}$ & $f_{*}$ & $f_{! !}$ & $f^{!}$ & $\underset{\lim }{\longrightarrow}$ & $\stackrel{\lim }{\leftarrow}$ \\
\hline$\iota$ & $\circ$ & $\circ$ & $\circ$ & $\times$ & $\circ$ & $\times$ & $\circ$ \\
\hline$\alpha$ & $\circ$ & $\circ$ & $\circ$ & $\circ$ & $\times$ & $\circ$ & $\circ$ \\
\hline$\beta$ & $\circ$ & $\circ$ & $\times$ & $\times$ & $\times$ & $\circ$ & $\times$ \\
\hline
\end{tabular}

Note that the pairs $\left(f^{-1}, \mathrm{R} f_{*}\right)$ and $\left(\mathrm{R} f_{! !}, f^{!}\right)$are pairs of adjoint functors. Finally, note that the functor $f^{!}$commutes with filtrant inductive limits (after taking the cohomology).

\subsection{Ring action}

We do not recall here the notion of a ring object $\mathcal{B}$ or a $\mathcal{B}$-module in a tensor category $\mathcal{S}$ (see $[\mathrm{KS} 01, \S 5.4]$ ). (In the sequel, we shall consider the tensor category I $\left(\mathbf{k}_{M}\right)$, see Lemma 2.2.2.) For such a ring object $\mathcal{B}$ in $\mathcal{S}$, we denote by $\operatorname{Mod}(\mathcal{B})$ the abelian category of $\mathcal{B}$-modules in $\mathcal{S}$ and by $\mathrm{D}^{\mathrm{b}}(\mathcal{B})$ its derived category. 


\subsection{Ring action}

We shall encounter the following situation. Let $\mathcal{A}$ be a sheaf of $\mathbf{k}$-algebras on $M$. Consider an object $\mathscr{M}$ of $\mathrm{I}\left(\mathbf{k}_{M}\right)$ together with a morphism of sheaves of k-algebras

$$
\mathcal{A} \rightarrow \mathscr{E}_{n} d_{\mathcal{I}\left(\mathbf{k}_{M}\right)}(\mathscr{M}) .
$$

In this case one says that $\mathscr{M}$ is an $\mathcal{A}$-module in $\mathrm{I}\left(\mathbf{k}_{M}\right)$. One denotes by

- $\mathrm{I}(\mathcal{A})$ the abelian category of $\mathcal{A}$-modules in $\mathrm{I}\left(\mathbf{k}_{M}\right)$,

- $\mathrm{D}^{\mathrm{b}}(\mathrm{I} \mathcal{A}):=\mathrm{D}^{\mathrm{b}}(\mathrm{I}(\mathcal{A}))$ its bounded derived category. We use similar notations with $\mathrm{D}^{\mathrm{b}}$ replaced with $\mathrm{D}^{+}, \mathrm{D}^{-}$and $\mathrm{D}$.

One shall not confuse the category $\mathrm{I}(\mathcal{A})$ with the category $\operatorname{Ind}\left(\operatorname{Mod}^{\mathrm{c}}(\mathcal{A})\right)$ of ind-objects in the category of sheaves of $\mathcal{A}$-modules with compact support, and we shall not confuse their derived categories.

If $\mathcal{A}$ is a sheaf of $\mathbf{k}$-algebras as above, then $\beta_{M} \mathcal{A}$ is a ring-object in the tensor category $\mathrm{I}\left(\mathbf{k}_{M}\right)$. Since

$$
\operatorname{Hom}_{\mathbf{k}_{M}}\left(\mathcal{A}, \mathscr{H} o m_{\mathrm{Ik}_{M}}(\mathscr{M}, \mathscr{M})\right) \simeq \operatorname{Hom}_{\mathrm{I}\left(\mathbf{k}_{M}\right)}\left(\beta_{M} \mathcal{A} \otimes \mathscr{M}, \mathscr{M}\right),
$$

we get equivalences of categories

$$
\operatorname{Mod}\left(\beta_{M} \mathcal{A}\right) \simeq \mathrm{I}(\mathcal{A}), \quad \mathrm{D}^{\mathrm{b}}\left(\beta_{M} \mathcal{A}\right) \simeq \mathrm{D}^{\mathrm{b}}(\mathrm{I} \mathcal{A}) .
$$

Remark 2.3.1. Our notations differ from those of [KS 01, §5.4, §5.5].

- For a ring object $\mathscr{B}$ in $\mathrm{I}\left(\mathbf{k}_{M}\right), \operatorname{Mod}(\mathscr{B})$ in our notation was denoted by $\mathrm{I}(\mathscr{B})$ in $[\mathrm{KS} 01]$.

- For a sheaf of rings $\mathcal{A}, \mathrm{I}(\mathcal{A})$ in our notation was denoted by $\mathrm{I}(\beta \mathcal{A})$ and $\operatorname{Ind}\left(\operatorname{Mod}^{\mathrm{c}}(\mathcal{A})\right)$ in our notation was denoted by $\mathrm{I}(\mathcal{A})$ in $[\mathrm{KS} 01]$.

See [KS01, Exe. 3.4, Def. 4.1.2, Def. 5.4.4, Exe. 5.3].

We have the quasi-commutative diagram

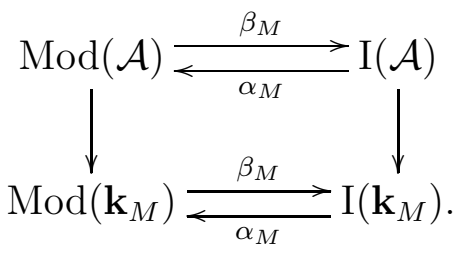




\subsection{Ring action}

For $\mathscr{M} \in \mathrm{D}^{\mathrm{b}}(\mathcal{A}), \mathscr{N} \in \mathrm{D}^{\mathrm{b}}\left(\mathcal{A}^{\mathrm{op}}\right)$ and $K \in \mathrm{D}^{\mathrm{b}}(\mathrm{I} \mathcal{A})$ one gets the objects, functorially in $\mathscr{M}, \mathscr{N}, K$ :

$$
\mathrm{R} \mathscr{H} o m_{\mathcal{A}}(\mathscr{M}, K) \in \mathrm{D}^{+}\left(\mathrm{Ik}_{M}\right), \quad \mathscr{N} \stackrel{\mathrm{L}}{\otimes}_{\mathcal{A}} K \in \mathrm{D}^{-}\left(\mathrm{Ik}_{M}\right) .
$$

They are characterized by

$$
\begin{aligned}
& \operatorname{Hom}_{\mathrm{D}\left(\mathrm{Ik}_{M}\right)}\left(L, \operatorname{R} \mathscr{H} o m_{\mathcal{A}}(\mathscr{M}, K)\right) \simeq \operatorname{Hom}_{\mathrm{D}(\mathcal{A})}\left(\mathscr{M}, \mathrm{R} \mathscr{H} o m_{\mathrm{Ik}_{M}}(L, K)\right), \\
& \operatorname{Hom}_{\mathrm{D}\left(\mathrm{Ik}_{M}\right)}\left(\mathscr{N} \stackrel{\mathrm{L}}{\otimes}_{\mathcal{A}} K, L\right) \simeq \operatorname{Hom}_{\mathrm{D}(\mathcal{A})}\left(\mathscr{N}, \mathrm{R} \mathscr{H}_{0 m_{\mathbf{I k}_{M}}}(K, L)\right)
\end{aligned}
$$

for any $L \in \mathrm{D}\left(\mathrm{Ik}_{M}\right)$.

Proposition 2.3.2. Let $\mathscr{M} \in \mathrm{D}^{\mathrm{b}}(\mathcal{A}), \mathscr{N} \in \mathrm{D}^{\mathrm{b}}\left(\mathcal{A}^{\mathrm{op}}\right)$ and $\mathscr{K} \in \mathrm{D}^{\mathrm{b}}(\mathrm{I} \mathcal{A})$. There are natural isomorphisms:

$$
\begin{aligned}
& \operatorname{R}{\mathscr{H} o m_{\mathcal{A}}}(\mathscr{M}, \mathscr{K}) \simeq \mathrm{R} \mathscr{I h o m}_{\beta_{M} \mathcal{A}}\left(\beta_{M} \mathscr{M}, \mathscr{K}\right) \text { in } \mathrm{D}^{+}\left(\mathrm{Ik}_{M}\right) \\
& \mathscr{N} \stackrel{\mathrm{L}}{\otimes}_{\mathcal{A}} \mathscr{K} \simeq \beta_{M} \mathscr{N} \stackrel{\mathrm{L}}{\otimes_{\beta_{M} \mathcal{A}}} \mathscr{K} \quad \text { in } \mathrm{D}^{-}\left(\mathrm{Ik}_{M}\right) .
\end{aligned}
$$

Proof. Let $L \in \mathrm{D}^{+}\left(\mathrm{Ik}_{M}\right)$. We have the sequence of isomorphisms

$$
\begin{aligned}
& \operatorname{Hom}_{\mathrm{D}\left(\mathbf{I k}_{M}\right)}\left(L, \operatorname{R}_{\mathcal{H}} o m_{\mathcal{A}}(\mathscr{M}, \mathscr{K})\right) \simeq \operatorname{Hom}_{\mathrm{D}(\mathcal{A})}\left(\mathscr{M}, \operatorname{R}_{\mathscr{H}} o m_{\mathbf{I k}_{M}}(L, \mathscr{K})\right) \\
& \simeq \operatorname{Hom}_{\mathrm{D}\left(\beta_{M} \mathcal{A}\right)}\left(\beta_{M} \mathscr{M}, \mathrm{R} \mathscr{I h o m}(L, \mathscr{K})\right) \\
& \simeq \operatorname{Hom}_{\mathrm{D}\left(\mathrm{Ik}_{M}\right)}\left(L, \mathrm{R} \mathscr{I} \operatorname{hom}_{\beta_{M \mathcal{A}}}\left(\beta_{M} \mathscr{M}, \mathscr{K}\right)\right) .
\end{aligned}
$$

The second formula is proved similarly.

Notation 2.3.3. For $\mathscr{M} \in \mathrm{D}^{\mathrm{b}}(\mathrm{I} \mathcal{A}), \mathscr{N} \in \mathrm{D}^{\mathrm{b}}\left(\mathrm{I} \mathcal{A}^{\mathrm{op}}\right)$ and $\mathscr{K} \in \mathrm{D}^{\mathrm{b}}(\mathrm{I} \mathcal{A})$, we shall use the notations $\mathrm{R} \mathscr{I}$ hom $_{\beta \mathcal{A}}(\mathscr{M}, \mathscr{K})$ and $\mathscr{N}{\stackrel{\mathrm{L}}{\otimes_{\beta \mathcal{A}}}} \mathscr{K}$, objects of $\mathrm{D}\left(\mathrm{Ik}_{M}\right)$.

Let us briefly recall a few basic formulas.

We consider the following situation: $f: M \rightarrow N$ is a continuous map of good topological spaces and $\mathscr{R}$ is a sheaf of $\mathbf{k}$-algebras on $N$.

In the sequel, $\mathrm{D}^{\dagger}$ is $\mathrm{D}, \mathrm{D}^{\mathrm{b}}, \mathrm{D}^{+}$or $\mathrm{D}^{-}$.

Theorem 2.3.4. (a) The functor $f^{-1}: \mathrm{I}\left(\mathbf{k}_{N}\right) \rightarrow \mathrm{I}\left(\mathbf{k}_{M}\right)$ induces a functor $f^{-1}: \mathrm{D}^{\dagger}(\mathrm{I} \mathscr{R}) \rightarrow \mathrm{D}^{\dagger}\left(\mathrm{I} f^{-1} \mathscr{R}\right)$. 
(b) The functor $f_{*}: \mathrm{I}\left(\mathbf{k}_{M}\right) \rightarrow \mathrm{I}\left(\mathbf{k}_{N}\right)$ induces a functor $\mathrm{R} f_{*}: \mathrm{D}^{\dagger}\left(\mathrm{I} f^{-1} \mathscr{R}\right) \rightarrow$ $\mathrm{D}^{\dagger}(\mathrm{I} \mathscr{R})$.

(c) The functor $f_{! !}: \mathrm{I}\left(\mathbf{k}_{M}\right) \rightarrow \mathrm{I}\left(\mathbf{k}_{N}\right)$ induces a functor $\mathrm{R} f_{! !}: \mathrm{D}^{\dagger}\left(\mathrm{I} f^{-1} \mathscr{R}\right) \rightarrow$ $\mathrm{D}^{\dagger}(\mathrm{I} \mathscr{R})$.

(d) the functor $\mathrm{R} f_{! !}: \mathrm{D}^{+}\left(\mathrm{I}^{-1} \mathscr{R}\right) \rightarrow \mathrm{D}^{+}(\mathrm{I} \mathscr{R})$ admits a a right adjoint, denoted by $f^{!}$.

Theorem 2.3.5. (a) For $G \in \mathrm{D}^{-}(\mathrm{I} \mathscr{R})$ and $F \in \mathrm{D}^{+}\left(\mathrm{I} f^{-1} \mathscr{R}\right)$, one has the isomorphism

$$
\mathrm{R} \mathscr{I h o m}_{\beta_{N} \mathscr{R}}\left(G, \mathrm{R} f_{*} F\right) \simeq \mathrm{R} f_{*} \mathrm{R} \mathscr{I} h o m_{f^{-1} \beta_{N} \mathscr{R}}\left(f^{-1} G, F\right) .
$$

(b) For $G \in \mathrm{D}^{+}(\mathrm{I} \mathscr{R})$ and $F \in \mathrm{D}^{-}\left(\mathrm{I}^{-1} \mathscr{R}\right)$, one has the isomorphism

$$
\mathrm{R} \mathscr{I h o m}_{\beta_{N} \mathscr{R}}\left(\mathrm{R} f_{! !} F, G\right) \simeq \mathrm{R} f_{*} \mathrm{R} \mathscr{I} h o m_{f^{-1} \beta_{N} \mathscr{R}}\left(F, f^{!} G\right) .
$$

(c) (Projection formula.) For $F \in \mathrm{D}^{-}\left(\mathrm{I}^{-1} \mathscr{R}\right)$ and $G \in \mathrm{D}^{-}\left(\mathrm{I} \mathscr{R}{ }^{\circ p}\right)$, one has the isomorphism

$$
G \stackrel{\mathrm{L}}{\otimes}_{\beta_{N} \mathscr{R}} \mathrm{R} f_{! !} F \simeq \mathrm{R} f_{! !}\left(f^{-1} G \stackrel{\mathrm{L}}{\otimes}_{f^{-1} \beta_{N} \mathscr{R}} F\right) .
$$

(d) (Base change formula.) Consider the Cartesian square of good topological spaces

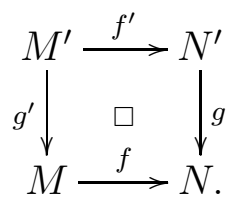

There are natural isomorphisms of functors from $\mathrm{D}^{\dagger}\left(\mathrm{I}^{-1} \mathscr{R}\right)$ to $\mathrm{D}^{\dagger}\left(\mathrm{I}^{-1} \mathscr{R}\right)$

$$
\begin{aligned}
& \mathrm{R} f^{\prime}{ }_{! !} g^{\prime-1} \simeq g^{-1} \mathrm{R} f_{! !}, \\
& \mathrm{R} f^{\prime}{ }_{*} g^{\prime !} \simeq g^{!} \mathrm{R} f_{*} .
\end{aligned}
$$

Note that Theorem 2.3.6 below has no counterpart in classical sheaf theory. 
Theorem 2.3.6. Let $\mathscr{A}$ be a sheaf of $\mathbf{k}_{M}$-algebras, let $F \in \mathrm{D}^{\mathrm{b}}\left(\mathbf{k}_{M}\right)$, let $\mathscr{K} \in \mathrm{D}^{\mathrm{b}}\left(\mathrm{I}^{\mathrm{op}}\right)$ and let $\mathscr{L} \in \mathrm{D}^{\mathrm{b}}(\mathcal{A})$. Then one has the isomorphism:

$$
\mathrm{R} \mathscr{I h o m}(F, \mathscr{K}) \stackrel{\mathrm{L}}{\otimes}_{\mathcal{A}} \mathscr{L} \stackrel{\mathrm{R}}{\longrightarrow} \operatorname{hhom}\left(F, \mathscr{K} \stackrel{\mathrm{L}}{\otimes}_{\mathcal{A}} \mathscr{L}\right) .
$$

Thanks to Proposition 2.3.2, isomorphism (2.3.5) may also be formulated as

$$
\mathrm{R} \mathscr{I h o m}(F, \mathscr{K}) \stackrel{\mathrm{L}}{\otimes}_{\beta_{M} \mathcal{A}} \beta_{M} \mathscr{L} \stackrel{\sim}{\sim} \operatorname{R} \mathscr{I h o m}\left(F, \mathscr{K} \stackrel{\mathrm{L}}{\otimes}_{\beta_{M} \mathcal{A}} \beta_{M} \mathscr{L}\right) .
$$

Also note that (2.3.5) is no more true if we relax the hypothesis that $F \in$ $\mathrm{D}^{\mathrm{b}}\left(\mathbf{k}_{M}\right)$.

\subsection{Sheaves on the subanalytic site}

Recall first that, for real analytic manifolds $M, N$ and a closed subanalytic subset $S$ of $M$, we say that a map $f: S \rightarrow N$ is subanalytic if its graph is subanalytic in $M \times N$. One denotes by $\mathscr{A}_{S}^{\mathbb{R}}$ the sheaf of continuous $\mathbb{R}$-valued subanalytic maps on $S$. A subanalytic space $\left(M, \mathscr{A}_{M}^{\mathbb{R}}\right)$, or simply $M$ for short, is an $\mathbb{R}$-ringed space locally isomorphic to $\left(S, \mathscr{A}_{S}^{\mathbb{R}}\right)$ for a closed subanalytic subset $S$ of a real analytic manifold. A morphism of subanalytic spaces is a morphism of $\mathbb{R}$-ringed spaces. Then we obtain the category of subanalytic spaces.

We can define the notion of subanalytic subsets of a subanalytic space, as well as $\mathbb{R}$-constructible sheaves on a subanalytic space.

Definition 2.4.1. Let $M$ be a subanalytic space, $\mathrm{Op}_{M}$ the category of its open subsets, the morphisms being the inclusion. One denotes by $\mathrm{Op}_{M_{\mathrm{sa}}}$ the full subcategory of $\mathrm{Op}_{M}$ consisting of subanalytic relatively compact open subsets. The site $M_{\mathrm{sa}}$ is obtained by deciding that a family $\left\{U_{i}\right\}_{i \in I}$ of subobjects of $U \in \mathrm{Op}_{M_{\mathrm{sa}}}$ is a covering of $U$ if there exists a finite subset $J \subset I$ such that $\bigcup_{j \in J} U_{j}=U$. One calls $M_{\text {sa }}$ the subanalytic site associated to $M$.

Note that

(2.4.1) $\left\{\begin{array}{c}\text { a presheaf } F \text { on } M_{\mathrm{sa}} \text { is a sheaf if and only if } F(\emptyset)=0 \text { and for any } \\ \text { pair }\left(U_{1}, U_{2}\right) \text { in } \mathrm{Op}_{M_{\mathrm{sa}}} \text {, the sequence below is exact: } \\ 0 \rightarrow F\left(U_{1} \cup U_{2}\right) \rightarrow F\left(U_{1}\right) \oplus F\left(U_{2}\right) \rightarrow F\left(U_{1} \cap U_{2}\right) .\end{array}\right.$ 
Let us denote by

$$
\rho_{M}: M \rightarrow M_{\mathrm{sa}}
$$

the natural morphism of sites and, as usual, by $\operatorname{Mod}\left(\mathbf{k}_{M_{\mathrm{sa}}}\right)$ the Grothendieck category of sheaves of $\mathbf{k}$-modules on $M_{\mathrm{sa}}$. Hence, $\left(\rho_{M}^{-1}, \rho_{M_{*}}\right)$ is a pair of adjoint functors.

The functor $\rho_{M}^{-1}$ also admits a left adjoint, denoted by $\rho_{M !}$. For $F \in$ $\operatorname{Mod}\left(\mathbf{k}_{M}\right), \rho_{M !} F$ is the sheaf associated to the presheaf $U \mapsto F(\bar{U}), U \in$ $\mathrm{Op}_{M_{\mathrm{sa}}}$. Hence we have the two pairs of adjoint functors $\left(\rho_{M}^{-1}, \rho_{M_{*}}\right)$ and $\left(\rho_{M !}, \rho_{M}^{-1}\right)$

$$
\operatorname{Mod}\left(\mathbf{k}_{M}\right) \underset{\rho_{M !}}{\stackrel{\rho_{M *}}{\longleftarrow}} \operatorname{\rho _{M}^{-1}\longrightarrow } \operatorname{Mod}\left(\mathbf{k}_{M_{\mathrm{sa}}}\right) .
$$

The functor $\rho_{M *}$ is fully faithful.

One denotes by "lim" the inductive limit in the category $\operatorname{Mod}\left(\mathbf{k}_{M_{\mathrm{sa}}}\right)$. Inductive limits do not commute with the functor $\rho_{M *}$.

Remark 2.4.2. It would be possible to develop the theory of subanalytic sheaves, that is sheaves on the subanalytic site, and in particular the six operations (see $[\operatorname{Pr} 08])$. However, in these Notes, we prefer to embed the category of subanalytic sheaves into that of indsheaves, as we shall do now.

Denote by $\mathbb{R}-\mathrm{C}\left(\mathbf{k}_{M}\right)$ the small abelian category of $\mathbb{R}$-constructible sheaves (see [KS 90] for an exposition) and denote by $\mathbb{R}-\mathrm{C}^{\mathrm{c}}\left(\mathbf{k}_{M}\right)$ the full subcategory consisting of sheaves with compact support. Recall that $\mathrm{D}^{\mathrm{b}}\left(\mathbb{R}-\mathrm{C}\left(\mathbf{k}_{M}\right)\right) \simeq$ $\mathrm{D}_{\mathbb{R}-\mathrm{c}}^{\mathrm{b}}\left(\mathbf{k}_{M}\right)$. Set

$$
\mathrm{I}_{\mathbb{R}-\mathrm{c}}\left(\mathbf{k}_{M}\right)=\operatorname{Ind}\left(\mathbb{R}-\mathrm{C}^{\mathrm{c}}\left(\mathbf{k}_{M}\right)\right) .
$$

The fully faithful functor $\mathbb{R}-\mathrm{C}^{\mathrm{c}}\left(\mathbf{k}_{M}\right) \rightarrow \operatorname{Mod}^{\mathrm{c}}\left(\mathbf{k}_{M}\right)$ induces a fully faithful functor $\mathrm{I}_{\mathbb{R}-\mathrm{c}}\left(\mathbf{k}_{M}\right) \rightarrow \mathrm{I}\left(\mathbf{k}_{M}\right)$, by which we regard $\mathrm{I}_{\mathbb{R}-\mathrm{c}}\left(\mathbf{k}_{M}\right)$ as a full subcategory of $\mathrm{I}\left(\mathbf{k}_{M}\right)$.

We say that an indsheaf on $M$ is a subanalytic indsheaf if it is isomorphic to an object of $\mathrm{I}_{\mathbb{R}-\mathrm{c}}\left(\mathbf{k}_{M}\right)$.

We have a quasi-commutative diagram of categories in which all arrows are exact and fully faithful:

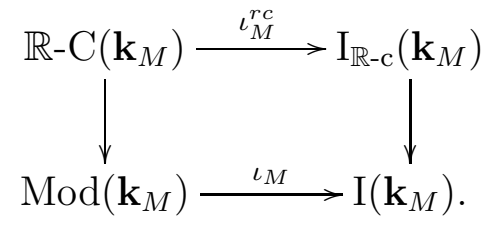


Proposition 2.4.3. The restriction of the functor $\rho_{M *}$ to the subcategory $\mathbb{R}-\mathrm{C}\left(\mathbf{k}_{M}\right)$ is exact and fully faithful.

We have a natural functor

$$
\lambda_{M}: \mathrm{I}_{\mathbb{R}-\mathrm{c}}\left(\mathbf{k}_{M}\right) \rightarrow \operatorname{Mod}\left(\mathbf{k}_{M_{\mathrm{sa}}}\right), \quad \underset{i}{\stackrel{\lim }{\longrightarrow}} F_{i} \mapsto \underset{i}{\stackrel{\lim }{\rightarrow}} \rho_{M_{*}} F_{i},
$$

where the first "lim" is taken in the category $\mathrm{I}_{\mathbb{R}-c}\left(\mathbf{k}_{M}\right)$ and the second one is taken in the category $\operatorname{Mod}\left(\mathbf{k}_{M_{\mathrm{sa}}}\right)$.

Theorem 2.4.4. The functor $\lambda_{M}$ in (2.4.4) is an equivalence.

In other words, subanalytic indsheaves are usual sheaves on the subanalytic site. By this result, the embedding $\mathrm{I}_{\mathbb{R}-\mathrm{c}}\left(\mathbf{k}_{M}\right) \hookrightarrow \mathrm{I}\left(\mathbf{k}_{M}\right)$ gives an exact and fully faithful functor

$$
\widetilde{\iota}_{M}: \operatorname{Mod}\left(\mathbf{k}_{M_{\mathrm{sa}}}\right) \rightarrow \mathrm{I}\left(\mathbf{k}_{M}\right) .
$$

Note that for $G \in \operatorname{Mod}\left(\mathbf{k}_{M_{\mathrm{sa}}}\right)$, one has

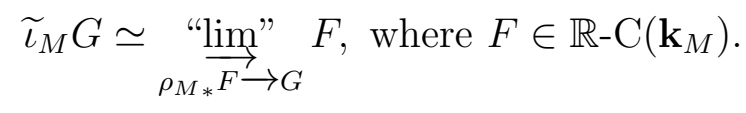

Also note that

$$
\widetilde{\iota}_{M} \mathscr{H} o m(F, G) \simeq \mathscr{I h o m}\left(F, \widetilde{\iota}_{M} G\right) \text { for } F \in \mathbb{R}-\mathrm{C}\left(\mathbf{k}_{M}\right), G \in \operatorname{Mod}\left(\mathbf{k}_{M_{\mathrm{sa}}}\right) .
$$

We have the following diagrams, where the one in the left is non commutative and the one in the right is commutative (see Diagram 2.1.1 for the case $M=\mathrm{pt})$ :
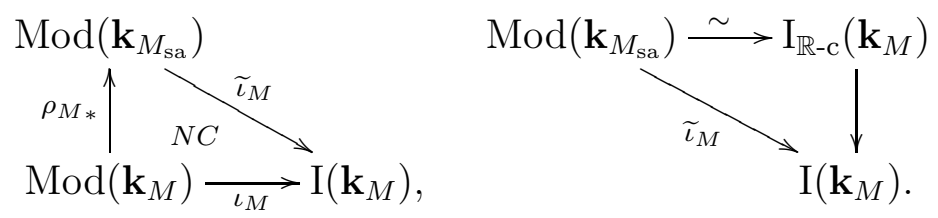

The functors $\iota_{M}$ and $\widetilde{\iota}_{M}$ are exact but $\rho_{M *}$ is not right exact in general.

Lemma 2.4.5. The two diagrams below commute:

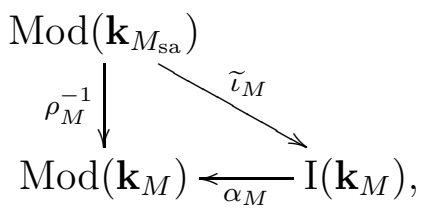

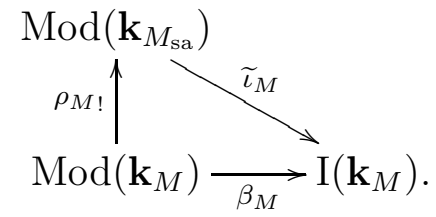


Proof. (i) Let us prove the commutation of the diagram on the left. Since all functors in the diagram commute with inductive limits, we are reduced to prove the isomorphism $\rho_{M}^{-1} \rho_{M *} F \simeq \alpha_{M} \widetilde{\iota}_{M} \rho_{M *} F$ for $F \in \mathbb{R}-\mathrm{C}^{\mathrm{c}}\left(\mathbf{k}_{M}\right)$ and the result is clear in this case.

(ii) Let us prove the commutation of the diagram on the right. Again all functors in the diagram commute with inductive limits. We shall first prove that

$$
\begin{aligned}
& \text { the functor } \beta_{M} \text { factors as } \beta_{M}=\widetilde{\iota}_{M} \circ \lambda_{M} \text { for a functor } \\
& \lambda_{M}: \operatorname{Mod}\left(\mathbf{k}_{M}\right) \rightarrow \operatorname{Mod}\left(\mathbf{k}_{M_{\mathrm{sa}}}\right) .
\end{aligned}
$$

First consider the case of $F=\mathbf{k}_{U}$ for $U$ open and relatively compact in $M$. In this case,

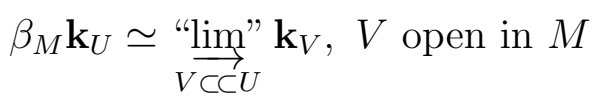

and we may assume that $V$ is subanalytic. Hence $\beta_{M} \mathbf{k}_{U}$ is a subanalytic indsheaf. Since any $F \in \operatorname{Mod}\left(\mathbf{k}_{M}\right)$ is obtained by taking direct sums and cokernels of sheaves of the type $\mathbf{k}_{U}$ and the subcategory of subanalytic indsheaves is stable by these operations, $\beta_{M} F$ is a subanalytic indsheaf for any $F \in \operatorname{Mod}\left(\mathbf{k}_{M}\right)$ and we get (2.4.8). It remains to prove that $\lambda_{M} \simeq \rho_{M !}$. Let $F \in \operatorname{Mod}\left(\mathbf{k}_{M}\right)$ and $G \in \operatorname{Mod}\left(\mathbf{k}_{M_{\mathrm{sa}}}\right)$. Using (i) and the fact that $\widetilde{\iota}_{M}$ is fully faithful, we have

$$
\begin{aligned}
\operatorname{Hom}\left(\rho_{M !} F, G\right) & \simeq \operatorname{Hom}\left(F, \rho_{M}^{-1} G\right) \simeq \operatorname{Hom}\left(F, \alpha_{M} \widetilde{\iota}_{M} G\right) \\
& \simeq \operatorname{Hom}\left(\beta_{M} F, \widetilde{\iota}_{M} G\right) \simeq \operatorname{Hom}\left(\widetilde{\iota}_{M} \lambda_{M} F, \widetilde{\iota}_{M} G\right) \\
& \simeq \operatorname{Hom}\left(\lambda_{M} F, G\right) .
\end{aligned}
$$

Q.E.D.

We denote by $\mathrm{D}_{\mathrm{IR}-\mathrm{c}}^{\mathrm{b}}\left(\mathrm{Ik}_{M}\right)$ the full subcategory of $\mathrm{D}^{\mathrm{b}}\left(\mathrm{Ik}_{M}\right)$ consisting of objects with subanalytic indsheaves as cohomologies. By [KS01, Th 7.1], we have:

Theorem 2.4.6. The functor $\widetilde{\iota}_{M}$ induces an equivalence of triangulated categories

$$
\mathrm{D}^{\mathrm{b}}\left(\mathbf{k}_{M_{\mathrm{sa}}}\right) \stackrel{\sim}{\sim} \mathrm{D}_{\mathrm{IR}-c}^{\mathrm{b}}\left(\mathrm{Ik}_{M}\right) .
$$

Proposition 2.4.7. Let $M$ be a subanalytic space. 
(i) Let $K, L \in \mathrm{D}_{\mathbb{I} \mathbb{R}-c}^{\mathrm{b}}\left(\mathrm{Ik}_{M}\right)$. Then $K \otimes L \in \mathrm{D}_{\mathbb{I R}-c}^{\mathrm{b}}\left(\mathrm{Ik}_{M}\right)$.

(ii) Let $K \in \mathrm{D}_{\mathbb{I}-c}^{\mathrm{b}}\left(\mathrm{Ik}_{M}\right)$ and let $F \in \mathrm{D}_{\mathbb{R}-c}^{\mathrm{b}}\left(\mathbf{k}_{M}\right)$. Then $\mathrm{R} \mathscr{I h o m}(F, K) \in$ $\mathrm{D}_{\mathrm{IR}-c}^{\mathrm{b}}\left(\mathrm{Ik}_{M}\right)$.

Proposition 2.4.8. Let $f: M \rightarrow N$ be a morphism of subanalytic spaces.

(i) For $L \in \mathrm{D}_{I \mathbb{R}-c}^{\mathrm{b}}\left(\mathrm{Ik}_{N}\right)$, we have $f^{-1} L \in \mathrm{D}_{\mathrm{IR}-c}^{\mathrm{b}}\left(\mathrm{Ik}_{M}\right)$ and $f^{!} L \in \mathrm{D}_{\mathrm{IR}-c}^{\mathrm{b}}\left(\mathrm{Ik}_{M}\right)$.

(ii) For $K \in \mathrm{D}_{\mathrm{IR}-c}^{\mathrm{b}}\left(\mathrm{Ik}_{M}\right)$, we have $\mathrm{R} f_{! !} K \in \mathrm{D}_{\mathrm{IR}-c}^{\mathrm{b}}\left(\mathrm{Ik}_{N}\right)$.

The next result will be of a constant use.

Proposition 2.4.9. A morphism $u: K \rightarrow L$ in $\mathrm{D}_{\mathrm{IR}-c}^{\mathrm{b}}\left(\mathrm{Ik}_{M}\right)$ is an isomorphism if and only if, for any relatively compact subanalytic open subset $U$ of $M$ and any $n \in \mathbb{Z}, u$ induces an isomorphism $\operatorname{Hom}_{\mathrm{D}^{\mathrm{b}\left(\mathrm{Ik}_{M}\right)}}\left(\mathbf{k}_{U}[n], K\right) \stackrel{\longrightarrow}{\longrightarrow}$ $\operatorname{Hom}_{\mathrm{D}^{\mathrm{b}}\left(\mathbf{I k}_{M}\right)}\left(\mathbf{k}_{U}[n], L\right)$.

\subsection{Some classical sheaves on the subanalytic site}

In this subsection, we take $\mathbb{C}$ as the base field $\mathbf{k}$.

Notation 2.5.1. Let $X$ be a complex manifold and let $\mathscr{D}_{X}$ be the sheaf of differential operators, as in $\S 1.2$. According to Proposition 2.3.2, for $\mathscr{M} \in \mathrm{D}^{\mathrm{b}}\left(\mathscr{D}_{X}\right)$, we get the functors

$$
\begin{array}{rll}
\mathrm{R} \mathscr{H}_{\mathscr{D}_{X}}(\mathscr{M}, \cdot) & : & \mathrm{D}^{\mathrm{b}}\left(\mathrm{I}_{X}\right) \rightarrow \mathrm{D}^{+}\left(\mathrm{IC}_{X}\right), \\
\cdot{ }^{\mathrm{L}} \mathscr{D}_{X} \mathscr{M} & : & \mathrm{D}^{\mathrm{b}}\left(\mathrm{I}_{X}^{\mathrm{op}}\right) \rightarrow \mathrm{D}^{-}\left(\mathrm{IC}_{X}\right), \\
\cdot \stackrel{\mathrm{D}}{\otimes}: & : \quad \mathrm{D}^{-}\left(\mathrm{I} \mathscr{D}_{X}\right) \times \mathrm{D}^{-}\left(\mathrm{I}_{X}\right) \rightarrow \mathrm{D}^{-}\left(\mathrm{I}_{X}\right) .
\end{array}
$$

There are similar constructions with right $\mathscr{D}_{X}$-modules.

If $M$ is a real analytic manifold, we denote by $\mathscr{D}_{M}$ the sheaf of finiteorder differential operators with real analytic coefficients. Denoting by $X$ a complexification of $M$, we have $\left.\mathscr{D}_{M} \simeq \mathscr{D}_{X}\right|_{M}$ and the notations above apply with $\mathscr{D}_{X}$ replaced by $\mathscr{D}_{M}$. 


\subsubsection{Tempered and Whitney functions and distributions}

In this subsection and the next ones, $M$ denotes a real analytic manifold.

As usual, we denote by $\mathcal{C}_{M}^{\infty}$ (resp. $\mathcal{C}_{M}^{\omega}$ ) the sheaf of $\mathbb{C}$-valued functions of class $\mathrm{C}^{\infty}$ (resp. real analytic) and by $\mathcal{D} b_{M}$ (resp. $\mathscr{B}_{M}$ ) the sheaf of Schwartz's distributions (resp. Sato's hyperfunctions). We also use the notation $\mathscr{A}_{M}=$ $\mathcal{C}_{M}^{\omega}$.

Definition 2.5.2. Let $U$ be an open subset of $M$ and $f \in \mathcal{C}_{M}^{\infty}(U)$. One says that $f$ has polynomial growth at $p \in M$ if $f$ satisfies the following condition: for a local coordinate system $\left(x_{1}, \ldots, x_{n}\right)$ around $p$, there exist a sufficiently small compact neighborhood $K$ of $p$ and a positive integer $N$ such that

$$
\sup _{x \in K \cap U}(\operatorname{dist}(x, K \backslash U))^{N}|f(x)|<\infty .
$$

Here, $\operatorname{dist}(x, K \backslash U):=\inf \{|y-x| ; y \in K \backslash U\}$, and we understand that the left-hand side of (2.5.1) is 0 if $K \cap U=\emptyset$ or $K \backslash U=\emptyset$. Hence $f$ has polynomial growth at any point of $U$. We say that $f$ is tempered at $p$ if all its derivatives have polynomial growth at $p$. We say that $f$ is tempered if it is tempered at any point of $M$.

An important property of subanalytic subsets is given by the lemma below. (See Lojasiewicz [Lo59] and also [Ma66] for a detailed study of its consequences.)

Lemma 2.5.3. Let $U$ and $V$ be two relatively compact open subanalytic subsets of $\mathbb{R}^{n}$. There exist a positive integer $N$ and $C>0$ such that

$$
\operatorname{dist}\left(x, \mathbb{R}^{n} \backslash(U \cup V)\right)^{N} \leq C\left(\operatorname{dist}\left(x, \mathbb{R}^{n} \backslash U\right)+\operatorname{dist}\left(x, \mathbb{R}^{n} \backslash V\right)\right) .
$$

For an open subanalytic subset $U$ in $M$, denote by $\mathcal{C}_{M}^{\infty, \mathrm{t}}(U)$ the subspace of $\mathcal{C}_{M}^{\infty}(U)$ consisting of tempered $\mathrm{C}^{\infty}$-functions.

Denote by $\mathcal{D} b_{M}^{\mathrm{t}}(U)$ the image of the restriction map $\Gamma\left(M ; \mathcal{D} b_{M}\right) \rightarrow$ $\Gamma\left(U ; \mathcal{D} b_{M}\right)$, and call it the space of tempered distributions on $U$. Using Lemma 2.5.3 and (2.4.1) one proves:

- the presheaf $U \mapsto \mathcal{C}_{M}^{\infty, \mathrm{t}}(U)$ is a sheaf on $M_{\mathrm{sa}}$,

- the presheaf $U \mapsto \mathcal{D} b_{M}^{\mathrm{t}}(U)$ is a sheaf on $M_{\mathrm{sa}}$. 
One denotes them by $\mathcal{C}_{M_{\mathrm{sa}}}^{\infty, \mathrm{t}}$ and $\mathcal{D} b_{M_{\mathrm{sa}}}^{\mathrm{t}}$.

For a closed subanalytic subset $S$ in $M$, denote by $\mathrm{I}_{M, S}^{\infty}$ the space of $\mathrm{C}^{\infty}$ functions defined on $M$ which vanish up to infinite order on $S$. In [KS 96], one introduced the sheaf:

$$
\mathbb{C}_{U} \stackrel{\mathrm{w}}{\otimes} \mathcal{C}_{M}^{\infty}:=V \longmapsto \mathrm{I}_{V, V \backslash U}^{\infty}
$$

and showed that it uniquely extends to an exact functor

$$
\text { - } \stackrel{\mathrm{w}}{\otimes} \mathscr{C}_{M}^{\infty}: \operatorname{Mod}_{\mathbb{R}-\mathrm{c}}\left(\mathbb{C}_{M}\right) \rightarrow \operatorname{Mod}\left(\mathbb{C}_{M}\right) .
$$

One denotes by $\mathcal{C}_{M_{\mathrm{sa}}}^{\infty, \mathrm{w}}$ the sheaf on $M_{\mathrm{sa}}$ given by

$$
\mathcal{C}_{M_{\mathrm{sa}}}^{\infty, \mathrm{w}}(U)=\Gamma\left(M ; H^{0}\left(\mathrm{D}_{M}^{\prime} \mathbf{k}_{U}\right) \stackrel{\mathrm{w}}{\otimes} \mathscr{C}_{M}^{\infty}\right), U \in \mathrm{Op}_{M_{\mathrm{sa}}} .
$$

If $\mathrm{D}_{M}^{\prime} \mathbb{C}_{U} \simeq \mathbb{C}_{\bar{U}}$, then $\mathcal{C}_{M_{\mathrm{sa}}}^{\infty, \mathrm{w}}(U) \simeq \mathcal{C}_{M}^{\infty}(M) / \mathrm{I}_{M, \bar{U}}^{\infty}$ is the space of Whitney functions on $\bar{U}$. It is thus natural to call $\mathcal{C}_{M_{\mathrm{sa}}}^{\infty, \mathrm{w}}$ the sheaf of Whitney $\mathrm{C}^{\infty}$ functions on $M_{\mathrm{sa}}$.

Note that the sheaf $\rho_{M_{*}} \mathscr{D}_{M}$ does not operate on the sheaves $\mathcal{C}_{M_{\mathrm{sa}}}^{\infty, \mathrm{t}}, \mathcal{D} b_{M_{\mathrm{sa}}}^{\mathrm{t}}$, $\mathcal{C}_{M_{\mathrm{sa}}}^{\infty, \mathrm{w}}$ but $\rho_{M !} \mathscr{D}_{M}$ does.

Notation 2.5.4. Recall the exact and fully faithful functor $\widetilde{\iota}_{M}: \operatorname{Mod}\left(\mathbb{C}_{M_{\mathrm{sa}}}\right) \rightarrow$ $\operatorname{Mod}\left(\mathbb{I}_{M}\right)$ in (2.4.5). We denote by $\mathcal{C}_{M}^{\infty, \mathrm{w}}, \mathcal{C}_{M}^{\infty, \mathrm{t}}$ and $\mathcal{D} b_{M}^{\mathrm{t}}$ the indsheaves $\widetilde{\iota}_{M} \mathcal{C}_{M_{\mathrm{sa}}}^{\infty, \mathrm{w}}, \widetilde{\iota}_{M} \mathcal{C}_{M_{\mathrm{sa}}}^{\infty, \mathrm{t}}$ and $\widetilde{\iota}_{M} \mathcal{D} b_{M_{\mathrm{sa}}}^{\mathrm{t}}$ and calls them the indsheaves of Whitney functions, tempered $\mathrm{C}^{\infty}$-functions and tempered distributions, respectively.

We have monomorphisms of indsheaves

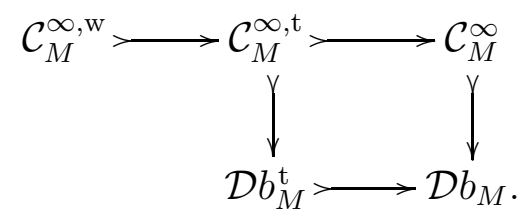

Let $F \in \mathrm{D}_{\mathbb{R}-\mathrm{c}}^{\mathrm{b}}\left(\mathbb{C}_{M}\right)$. One has the isomorphisms in $\mathrm{D}^{\mathrm{b}}\left(\mathbb{C}_{M}\right)$ :

$$
\begin{aligned}
\rho_{M}^{-1} \mathrm{R} \mathscr{H} O m\left(\mathrm{R} \rho_{M_{*}} F, \mathcal{D} b_{M_{\mathrm{sa}}}^{\mathrm{t}}\right) & \simeq \operatorname{R} \mathscr{H} o m_{\mathrm{IC}_{M}}\left(F, \mathcal{D} b_{M}^{\mathrm{t}}\right) \\
& \simeq \operatorname{Thom}\left(F, \mathcal{D} b_{M}\right),
\end{aligned}
$$

where the functor

$$
\operatorname{Thom}\left(\cdot, \mathcal{D} b_{M}\right): \mathrm{D}_{\mathbb{R}-\mathrm{c}}^{\mathrm{b}}\left(\mathbb{C}_{M}\right)^{\mathrm{op}} \rightarrow \mathrm{D}^{\mathrm{b}}\left(\mathbb{C}_{M}\right)
$$


was defined in [Ka80, Ka84] as the main tool for the proof of the RiemannHilbert correspondence for regular holonomic D-modules.

We also have

$$
\mathrm{R} \mathscr{H} O m_{\mathrm{IC}_{M}}\left(F, \mathcal{C}_{M}^{\infty, \mathrm{w}}\right) \simeq \mathrm{D}_{M}^{\prime} F \stackrel{\mathrm{w}}{\otimes} \mathcal{C}_{M}^{\infty} .
$$

We shall see in Subsection 2.5.4 that there is a kind of duality between the indsheaves $\mathcal{C}_{M}^{\infty, \mathrm{w}}$ and $\mathcal{D} b_{M}^{\mathrm{t}}$.

\subsubsection{Operations on tempered distributions}

Let us describe without detailed proofs the behaviour of the indsheaf of tempered distributions with respect to direct and inverse images (see [KS 01]). In [KS 96] these operations are treated in the language of the functor Thom introduced in [Ka 84], but we prefer to use the essentially equivalent language of indsheaves.

For a real analytic manifold $M$ and for a morphism of real analytic manifolds $f: M \rightarrow N$, we denote by

- $\operatorname{dim} M$ the dimension of $M$,

- $\mathscr{A}_{M}^{(\operatorname{dim} M)}$ the sheaf of real analytic forms of top degree,

- $\Theta_{M}$ the sheaf of real analytic vector fields,

- or $_{M}$ the orientation sheaf,

- $\mathscr{V}_{M}:=\mathscr{A}_{M}^{(\operatorname{dim} M)} \otimes$ or $_{M}$ the sheaf of real analytic densities on $M$,

- $\mathcal{D} b_{M}^{\mathrm{tv}}:=\mathscr{V}_{M} \stackrel{\mathrm{L}}{\otimes} \mathscr{\mathscr { A }}_{M} \mathcal{D} b_{M}^{\mathrm{t}}$ the indsheaf of tempered distributions densities,

- $\mathscr{D}_{M \rightarrow N}=\mathscr{A}_{M} \otimes_{f^{-1} \mathscr{A}_{N}} f^{-1} \mathscr{D}_{N}$ the transfer bimodule.

Proposition 2.5.5. Let $M$ and $N$ be two real analytic manifolds. There exists a natural morphism

$$
\mathcal{D} b_{M}^{\mathrm{t}} \otimes \mathcal{D} b_{N}^{\mathrm{t}} \rightarrow \mathcal{D} b_{M \times N}^{\mathrm{t}} \text { in } \mathrm{D}^{\mathrm{b}}\left(\mathrm{I}\left(\mathscr{D}_{M} \otimes \mathscr{D}_{N}\right)\right) .
$$

The next result is a feormulation of a theorem of [Ka 84]. 
Theorem 2.5.6. Let $f: M \rightarrow N$ be a morphism of real analytic manifolds. There exists a natural isomorphism

$$
\mathcal{D} b_{M}^{\mathrm{tv}} \stackrel{\mathrm{L}}{\otimes}_{\mathscr{D}_{M}} \mathscr{D}_{M \rightarrow N} \stackrel{\sim}{\longrightarrow} f^{!} \mathcal{D} b_{N}^{\mathrm{tv}} \text { in } \mathrm{D}^{\mathrm{b}}\left(\mathrm{I}^{-1} \mathscr{D}_{N}^{\mathrm{op}}\right) .
$$

Sketch of proof. (i) First, we construct the morphism in (2.5.4). By adjunction it is enough to construct a morphism

$$
\mathrm{R} f_{! !}\left(\mathcal{D} b_{M}^{\mathrm{tv}} \stackrel{\mathrm{L}}{\otimes}_{\mathscr{D}_{M}} \mathscr{D}_{M \rightarrow N}\right) \rightarrow \mathcal{D} b_{N}^{\mathrm{tv}} .
$$

Denote by $\operatorname{Sp} \cdot(\mathscr{M})$ the Spencer complex of a coherent $\mathscr{D}_{M}$-module $\mathscr{M}$. There is a quasi-isomorphism $\mathrm{Sp} .(\mathscr{M}) \rightarrow \mathscr{M}$, where $\operatorname{Sp}_{k}(\mathscr{M})$ is the $\mathscr{D}_{M^{-}}$ module $\mathscr{D}_{M} \otimes_{\mathscr{A}_{M}} \wedge^{k} \Theta_{M} \otimes_{\mathscr{A}_{M}} \mathscr{M}$. Then $\operatorname{Sp} \cdot\left(\mathscr{D}_{M \rightarrow N}\right)$ gives a resolution of $\mathscr{D}_{M \rightarrow N}$ as a $\left(\mathscr{D}_{M}, f^{-1} \mathscr{D}_{N}\right)$-bimodule locally free over $\mathscr{D}_{M}$. Note that $\mathcal{D} b_{M}^{\mathrm{tv}} \otimes_{\mathscr{D}_{M}} \operatorname{Sp}_{k}\left(\mathscr{D}_{M \rightarrow N}\right)$ is acyclic with respect to the functor $f_{! !}$for any $k$. Hence, in order to construct morphism (2.5.5), it is enough to construct a morphism of complexes in $\mathrm{I}\left(\mathscr{D}_{X}\right)$

$$
f_{! !}\left(\mathcal{D} b_{M}^{\mathrm{tv}} \otimes_{\mathscr{D}_{M}} \operatorname{Sp} \bullet\left(\mathscr{D}_{M \rightarrow N}\right)\right) \rightarrow \mathcal{D} b_{N}^{\mathrm{tV}} .
$$

Set for short

$$
\mathscr{K}_{\bullet}=\mathcal{D} b_{M}^{\mathrm{tv}} \otimes_{\mathscr{D}_{M}} \operatorname{Sp} \cdot\left(\mathscr{D}_{M \rightarrow N}\right) \simeq \mathcal{D} b_{M}^{\mathrm{tv}} \otimes_{\mathscr{A}_{M}} \dot{\bigwedge} \Theta_{M} \otimes_{f^{-1} \mathscr{A}_{N}} f^{-1} \mathscr{D}_{N} .
$$

Then we have $f_{! !}\left(\mathscr{K}_{0}\right)=f_{! !}\left(\mathcal{D} b_{M}^{\mathrm{tv}}\right) \otimes_{\mathscr{A}_{N}} \mathscr{D}_{N}$. The integration of distributions gives a morphism

$$
\int_{f}: f_{! !}\left(\mathcal{D} b_{M}^{\mathrm{tv}}\right) \rightarrow \mathcal{D} b_{N}^{\mathrm{t} v}
$$

Since $\mathcal{D} b_{N}^{\mathrm{tv}}$ is a right $\mathscr{D}_{N}$-module, we obtain the morphism $u: f_{! !}\left(\mathscr{K}_{0}\right) \rightarrow \mathcal{D} b_{N}^{\mathrm{tv}}$. By an explicit calculation, one checks that the composition

$$
f_{! !}\left(\mathscr{K}_{1}\right) \stackrel{d_{1}}{\longrightarrow} f_{! !} \mathscr{K}_{0} \stackrel{u}{\longrightarrow} \mathcal{D} b_{N}^{\mathrm{tv}}
$$

vanishes. This defines morphism (2.5.5) and hence the morphism in (2.5.4).

(ii) One can treat separately the case of a closed embedding and a submersion.

(a) If $f: M \rightarrow N$ is a closed embedding, the result follows from the isomorphism

$$
\operatorname{R} \mathscr{I h o m}\left(f_{*} \mathbb{C}_{M}, \mathcal{D} b_{N}^{\mathrm{t} \vee}\right) \simeq \mathcal{D} b_{M}^{\mathrm{t} \vee} \otimes_{\mathscr{D}_{M}} \mathscr{D}_{M \rightarrow N} .
$$


(b) When $f$ is a submersion, one reduces to the case where $M=N \times \mathbb{R}$ and $f$ is the projection. Let $F \in \mathrm{D}_{\mathbb{R}-\mathrm{c}}^{\mathrm{b}}\left(\mathbf{k}_{M}\right)$ such that $f$ is proper on $\operatorname{Supp}(F)$ and let us apply the functor $\mathrm{R} f_{*} \mathrm{R} \mathscr{H}$ om $(F, \bullet)$ to the morphism (2.5.4). Using $\mathrm{R} \mathscr{H} \circ{ }^{\prime}(F, \bullet) \simeq \alpha_{M} \circ \operatorname{R} \mathscr{F h o m}(F, \bullet)$, we get the morphism

$$
\begin{aligned}
\mathrm{R} f_{*}\left(\mathrm{R} \mathscr{H} o m\left(F, \mathcal{D} b_{M}^{\mathrm{tv}}\right) \stackrel{\mathrm{L}}{\otimes_{\mathscr{D}_{M}}} \mathscr{D}_{M \rightarrow N}\right) & \rightarrow \mathrm{R} f_{*} \mathrm{R} \mathscr{H} o m\left(F, f^{!} \mathcal{D} b_{N}^{\mathrm{tv}}\right) \\
& \simeq \mathrm{R} \mathscr{H} o m\left(\mathrm{R} f_{!} F, \mathcal{D} b_{N}^{\mathrm{tv}}\right) .
\end{aligned}
$$

By Proposition 2.4.9, it remains to prove that (2.5.8) is an isomorphism.

One then reduces to the case where $F=\mathbb{C}_{Z}$ for a closed subanalytic subset $Z$ of $N \times \mathbb{R}$ proper over $N$. Then, by using the structure of subanalytic sets, one reduces to the case where $f^{-1}(x) \cap Z$ is a closed interval for each $x \in f(Z)$. Finally, one proves that the sequence below is exact.

$$
0 \longrightarrow f_{!} \Gamma_{Z} \mathcal{D} b_{M} \stackrel{\partial_{t}}{\longrightarrow} f_{!} \Gamma_{Z} \mathcal{D} b_{M} \stackrel{\int_{\mathbb{R}}(\cdot) d t}{\longrightarrow} \Gamma_{f(Z)} \mathcal{D} b_{N} \rightarrow 0 .
$$

Q.E.D.

One often needs to compactify real analytic manifolds. In order to check that the construction does not depend on the choice of compactifications, the next lemma is useful.

Lemma 2.5.7. Consider a morphism $f: M \rightarrow N$ of real analytic manifolds and let $V \subset N$ be a subanalytic open subset. Set $U=f^{-1} V$ and assume that $f$ induces an isomorphism of real analytic manifolds $U \stackrel{\sim}{\longrightarrow}$. Then

$$
\operatorname{R} \mathscr{I h o m}\left(\mathbb{C}_{U}, \mathcal{D} b_{M}^{\mathrm{t}}\right) \simeq f^{!} \mathrm{R} \mathscr{I h o m}\left(\mathbb{C}_{V}, \mathcal{D} b_{N}^{\mathrm{t}}\right) .
$$

Proof. By Theorem 2.5.6, we have

$$
\begin{aligned}
& f^{!} \mathrm{R} \mathscr{I h o m}\left(\mathbb{C}_{V}, \mathcal{D} b_{N}^{\mathrm{t}}\right) \simeq \mathrm{R} \mathscr{I h o m}\left(f^{-1} \mathbb{C}_{V}, f^{!} \mathcal{D} b_{N}^{\mathrm{t}}\right) \\
& \simeq \operatorname{R} \mathscr{I h o m}\left(\mathbb{C}_{U}, \mathcal{D} b_{M}^{\mathrm{tv}} \stackrel{\mathrm{L}}{\otimes}_{\mathscr{D}_{M}} \mathscr{D}_{M \rightarrow N}\right) \text {. }
\end{aligned}
$$

Since the morphism of $\mathscr{D}_{M}$-modules $\mathscr{D}_{M} \rightarrow \mathscr{D}_{M \rightarrow N}$ is an isomorphism on $U$, it induces an isomorphism

$$
\mathrm{R} \mathscr{I h o m}\left(\mathbb{C}_{U}, \mathcal{D} b_{M}^{\mathrm{tv}} \stackrel{\mathrm{L}}{\otimes_{\mathscr{D}_{M}}} \mathscr{D}_{M}\right) \stackrel{\sim}{\sim} \mathscr{I h o m}\left(\mathbb{C}_{U}, \mathcal{D} b_{M}^{\mathrm{tv}} \stackrel{\mathrm{L}}{\otimes}_{\mathscr{D}_{M}} \mathscr{D}_{M \rightarrow N}\right) \text {. }
$$

Q.E.D. 
Remark 2.5.8. By choosing $N=$ pt and $F=\mathbb{C}_{U}$ for $U$ open subanalytic, we obtain that $\mathrm{RHom}\left(\mathrm{R} f \mathbb{C}_{U}, \mathbb{C}\right) \simeq \mathrm{R} \Gamma\left(U ; \omega_{M}\right)$ is isomorphic to the de Rham complex with coefficients in $\mathcal{D} b_{M}^{\mathrm{t}}(U)$. This is a vast generalization of a wellknown theorem of Grothendieck [Gr 66] which asserts that the cohomology of the complementary of an algebraic hypersurface $S$ may be calculated as the de Rham complex with coefficients in the sheaf of meromorphic functions with poles on $S$. This result has been generalized to the semi-analytic setting by Poly [Po74].

\subsubsection{Whitney and tempered holomorphic functions}

Let $X$ be a complex manifold. We denote by $X^{c}$ the complex conjugate manifold to $X$ and by $X_{\mathbb{R}}$ the underlying real analytic manifold.

We define the following indsheaves

$$
\begin{aligned}
\mathscr{O}_{X}^{\omega} & :=\beta_{X} \mathscr{O}_{X}, \\
\mathscr{O}_{X}^{\mathrm{w}} & :=\mathrm{R} \mathscr{H} O m_{\mathscr{D}_{X^{c}}}\left(\mathscr{O}_{X^{c}}, \mathcal{C}_{X_{\mathbb{R}}}^{\infty, \mathrm{w}}\right) \simeq \Omega_{X^{c}}{\stackrel{\mathrm{L}}{\mathscr{D}_{X}}}_{\mathcal{X}^{c}} \mathcal{C}_{X_{\mathbb{R}}}^{\infty, \mathrm{w}}\left[-d_{X}\right], \\
\mathscr{O}_{X}^{\mathrm{t}} & :=\mathrm{R} \mathscr{H} O m_{\mathscr{D}_{X^{c}}}\left(\mathscr{O}_{X^{c}}, \mathcal{D} b_{X_{\mathbb{R}}}^{\mathrm{t}}\right) \simeq \Omega_{X^{c}} \stackrel{\mathrm{L}}{\otimes}_{\mathscr{D}_{X^{c}}} \mathcal{D} b_{X_{\mathbb{R}}}^{\mathrm{t}}\left[-d_{X}\right], \\
\Omega_{X}^{\mathrm{t}} & :=\Omega_{X} \otimes_{\mathscr{O}_{X}} \mathscr{O}_{X}^{\mathrm{t}} .
\end{aligned}
$$

The first three are objects of $\mathrm{D}_{\mathrm{IR}-\mathrm{c}}^{\mathrm{b}}\left(\mathrm{I}_{X}\right)$ while the last one is an object of $\mathrm{D}_{\text {IR-c }}^{\mathrm{b}}\left(\mathrm{I} \mathscr{D}_{X}^{\mathrm{op}}\right)$. Hence $\mathscr{O}_{X}^{\mathrm{t}}$ is isomorphic to the Dolbeault complex with coefficients in $\mathcal{D} b_{X_{\mathbb{R}}}^{\mathrm{t}}$ :

$$
0 \longrightarrow \mathcal{D} b_{X_{\mathbb{R}}}^{\mathrm{t}} \stackrel{\bar{\partial}}{\longrightarrow} \mathcal{D} b_{X_{\mathbb{R}}}^{\mathrm{t}(0,1)} \stackrel{\bar{\partial}}{\longrightarrow} \cdots \stackrel{\bar{\partial}}{\longrightarrow} \mathcal{D} b_{X_{\mathbb{R}}}^{\mathrm{t}\left(0, d_{X}\right)} \longrightarrow 0,
$$

where $\mathcal{D} b_{X_{\mathbb{R}}}^{\mathrm{t}(0, p)}:=\Omega_{X^{c}}^{p} \otimes_{\mathscr{O}_{X c}} \mathcal{D} b_{X_{\mathbb{R}}}^{\mathrm{t}}$ is situated in degree $p$.

One calls $\mathscr{O}_{X}^{\mathrm{w}}$ and $\mathscr{O}_{X}^{\mathrm{t}}$ the indsheaves of Whitney and tempered holomorphic functions, respectively. We have the morphisms in the category $\mathrm{D}^{\mathrm{b}}\left(\mathrm{I} \mathscr{D}_{X}\right)$ :

$$
\mathscr{O}_{X}^{\omega} \rightarrow \mathscr{O}_{X}^{\mathrm{w}} \rightarrow \mathscr{O}_{X}^{\mathrm{t}} \rightarrow \mathscr{O}_{X}
$$

One proves the isomorphism

$$
\mathscr{O}_{X}^{\mathrm{t}} \simeq \mathrm{R} \mathscr{H}_{o m_{\mathscr{D}_{X}}}\left(\mathscr{O}_{X^{c}}, \mathcal{C}_{X_{\mathbb{R}}}^{\infty, \mathrm{t}}\right) \text { in } \mathrm{D}^{\mathrm{b}}\left(\mathrm{I} \mathscr{D}_{X}\right) \text {. }
$$

Note that the object $\mathscr{O}_{X}^{\mathrm{t}}$ is not concentrated in degree zero if $d_{X}>1$. Indeed, with the subanalytic topology, only finite coverings are allowed. If 
one considers for example the open subset $U \subset \mathbb{C}^{n}$, the difference of an open ball of radius $R$ and a closed ball of radius $r$ with $0<r<R$, then the Dolbeault complex will not be exact after any finite covering.

Example 2.5.9. (i) Let $Z$ be a closed complex analytic subset of the complex manifold $X$. We have the isomorphisms in $\mathrm{D}^{\mathrm{b}}\left(\mathscr{D}_{X}\right)$ :

$$
\begin{array}{ll}
\mathrm{R} \mathscr{H} o m_{\mathrm{IC}_{X}}\left(\mathrm{D}_{X}^{\prime} \mathbb{C}_{Z}, \mathscr{O}_{X}^{\omega}\right) \simeq\left(\mathscr{O}_{X}\right)_{Z} & \text { (restriction), } \\
\mathrm{R} \mathscr{H} o m_{\mathrm{IC}_{X}}\left(\mathrm{D}_{X}^{\prime} \mathbb{C}_{Z}, \mathscr{O}_{X}^{\mathrm{w}}\right) \simeq \mathscr{O}_{X} \widehat{\mid}_{Z} & (\text { formal completion) }, \\
\mathrm{R} \mathscr{H} o m_{\mathrm{IC}_{X}}\left(\mathbb{C}_{Z}, \mathscr{O}_{X}^{\mathrm{t}}\right) \simeq \mathrm{R} \Gamma_{[Z]}\left(\mathscr{O}_{X}\right) & \text { (algebraic cohomology) }, \\
\mathrm{R} \mathscr{H} o m_{\mathrm{IC}_{X}}\left(\mathbb{C}_{Z}, \mathscr{O}_{X}\right) \simeq \mathrm{R} \Gamma_{Z}\left(\mathscr{O}_{X}\right) & \text { (local cohomology). }
\end{array}
$$

(ii) Let $M$ be a real analytic manifold such that $X$ is a complexification of $M$. We have the isomorphisms in $\mathrm{D}^{\mathrm{b}}\left(\mathscr{D}_{M}\right)$ :

$$
\begin{array}{ll}
\left.\mathrm{R} \mathscr{H} o m_{\mathrm{IC}_{X}}\left(\mathrm{D}_{X}^{\prime} \mathbb{C}_{M}, \mathscr{O}_{X}^{\omega}\right)\right|_{M} \simeq \mathscr{A}_{M} & \text { (real analytic functions) } \\
\left.\mathrm{R} \mathscr{H} o m_{\mathrm{IC}_{X}}\left(\mathrm{D}_{X}^{\prime} \mathbb{C}_{M}, \mathscr{O}_{X}^{\mathrm{w}}\right)\right|_{M} \simeq \mathcal{C}_{M}^{\infty} & \left(\mathrm{C}^{\infty} \text {-functions) },\right. \\
\left.\mathrm{R} \mathscr{H} o m_{\mathrm{IC}_{X}}\left(\mathrm{D}_{X}^{\prime} \mathbb{C}_{M}, \mathscr{O}_{X}^{\mathrm{t}}\right)\right|_{M} \simeq \mathcal{D} b_{M} & \text { (distributions), } \\
\left.\mathrm{R} \mathscr{H} o m_{\mathrm{IC}_{X}}\left(\mathrm{D}_{X}^{\prime} \mathbb{C}_{M}, \mathscr{O}_{X}\right)\right|_{M} \simeq \mathscr{B}_{M} & \text { (hyperfunctions). }
\end{array}
$$

\subsubsection{Duality between Whitney and tempered functions}

We shall use the theory of topological $\mathbb{C}$-vector spaces of type FN (Fréchet nuclear spaces) or DFN (dual of Fréchet nuclear spaces). The categories of FN spaces and DFN spaces are quasi-abelian and the topological duality functor induces a contravariant equivalence between the category of FN spaces and DFN spaces. It induces therefore an equivalence of triangulated categories

$$
\mathrm{D}^{\mathrm{b}}(\mathrm{FN})^{\mathrm{op}} \simeq \mathrm{D}^{\mathrm{b}}(\mathrm{DFN}) .
$$

Proposition 2.5.10 ([KS96, Prop. 2.2]). Let $M$ be a real analytic manifold and let $F \in \mathbb{R}-\mathrm{C}\left(\mathbb{C}_{M}\right)$. Then, there exist natural topologies of type $\mathrm{FN}$ on $\Gamma\left(M ; F \stackrel{\mathrm{w}}{\otimes} \mathcal{C}_{M}^{\infty}\right)$ and of type DFN on $\Gamma_{c}\left(M ; \mathscr{H}{ }_{0} m_{\mathbb{I C}_{M}}\left(F, \mathcal{D} b_{M}^{\mathrm{tv}}\right)\right)$, and they are dual to each other.

Here, as usual, $\Gamma_{c}(M ; \bullet)$ is the functor of global sections with compact support. 
Hence for any open subset $U$ of $M$, we have

$$
\begin{aligned}
& \Gamma\left(U ; F \stackrel{\mathrm{w}}{\otimes} \mathcal{C}_{M}^{\infty}\right) \longrightarrow \operatorname{Hom}_{\mathbb{C}}\left(\Gamma_{c}\left(U ; \mathscr{H}^{\infty} m_{\mathrm{IC}_{M}}\left(F, \mathcal{D} b_{M}^{\mathrm{tv}}\right)\right), \mathbb{C}\right) \\
& \simeq \Gamma\left(U ; \mathrm{D}_{M} \mathscr{H} O m_{\mathrm{IC}_{M}}\left(F, \mathcal{D} b_{M}^{\mathrm{tv}}\right)\right),
\end{aligned}
$$

which induces a morphism of sheaves $F \stackrel{\mathrm{w}}{\otimes} \mathcal{C}_{M}^{\infty} \rightarrow \mathrm{D}_{M} \mathscr{H}_{0} m_{\mathrm{IC}_{M}}\left(F, \mathcal{D} b_{M}^{\mathrm{tv}}\right)$ and then a pairing

$$
\left(F \stackrel{\mathrm{w}}{\otimes} \mathcal{C}_{M}^{\infty}\right) \otimes \mathscr{H}_{0} m_{\mathbb{I C}_{M}}\left(F, \mathcal{D} b_{M}^{\mathrm{tv}}\right) \rightarrow \omega_{M} .
$$

Let $X$ be a complex manifold, let $\mathscr{M} \in \mathrm{D}_{\text {coh }}^{\mathrm{b}}\left(\mathscr{D}_{X}\right)$ and let $F, G \in \mathrm{D}_{\mathbb{R}-\mathrm{c}}^{\mathrm{b}}\left(\mathbb{C}_{X}\right)$. Set for short

$$
\begin{aligned}
\mathscr{W}(\mathscr{M}, F) & :=\operatorname{R} \mathscr{H} o m_{\mathscr{D}_{X}}\left(\mathscr{M}, F \stackrel{\mathrm{w}}{\otimes} \mathscr{O}_{X}\right), \\
\mathscr{T}(F, \mathscr{M}) & :=\operatorname{R} \mathscr{H} o m_{\mathrm{IC}_{X}}\left(F, \Omega_{X}^{\mathrm{t}}\left[d_{X}\right]\right) \stackrel{\mathrm{L}}{\otimes}_{\mathscr{D}_{X}} \mathscr{M} \\
W(G, \mathscr{M}, F) & :=\operatorname{RHom}\left(G, \operatorname{R} \mathscr{H} O m_{\mathscr{D}_{X}}\left(\mathscr{M}, F \mathscr{\mathrm { O }}_{X}\right)\right), \\
T_{c}(F, \mathscr{M}, G) & :=\operatorname{R} \Gamma_{c}\left(X ; \mathrm{R} \mathscr{H} o m_{\mathrm{IC}_{X}}\left(F, \Omega_{X}^{\mathrm{t}}\left[d_{X}\right]\right) \stackrel{\mathrm{L}}{\otimes}_{\mathscr{D}_{X}} \mathscr{M} \otimes G\right) .
\end{aligned}
$$

Then (2.5.11) induces a pairing

$$
\mathscr{W}(\mathscr{M}, F) \otimes \mathscr{T}(F, \mathscr{M}) \rightarrow \omega_{X} .
$$

and a pairing

$$
W(G, \mathscr{M}, F) \otimes T_{c}(F, \mathscr{M}, G) \rightarrow \mathbb{C},
$$

Theorem 2.5.11 ([KS96, Theorem 6.1]). The two objects $W(G, \mathscr{M}, F)$ and $T_{c}(F, \mathscr{M}, G)$ are well-defined in the categories $\mathrm{D}^{\mathrm{b}}(\mathrm{FN})$ and $\mathrm{D}^{\mathrm{b}}(\mathrm{DFN})$, respectively, and are dual to each other through the pairing (2.5.13).

Now we assume that $\mathscr{M} \in \mathrm{D}_{\text {hol }}^{\mathrm{b}}\left(\mathscr{D}_{X}\right)$ and we consider the following asser- 
tions.

(a) the object $\mathscr{W}(\mathscr{M}, F):=\mathrm{R} \mathscr{H} o m_{\mathscr{D}_{X}}\left(\mathscr{M}, F \stackrel{\mathrm{w}}{\otimes} \mathscr{O}_{X}\right)$ is $\mathbb{R}$ constructible,

(b) the object $\mathscr{T}(F, \mathscr{M}):=\mathrm{R} \mathscr{H} o m_{\mathrm{IC}_{X}}\left(F, \Omega_{X}^{\mathrm{t}}\left[d_{X}\right]\right) \stackrel{\mathrm{L}}{\otimes}_{\mathscr{D}_{X}} \mathscr{M}$ is $\mathbb{R}$-constructible,

(c) conditions (a) and (b) are satisfied, and the two complexes in (a) and (b) are dual to each other in the category $\mathrm{D}_{\mathbb{R}-\mathrm{c}}^{\mathrm{b}}\left(\mathbb{C}_{X}\right)$, that is, $\mathscr{W}(\mathscr{M}, F) \simeq \mathrm{D}_{X} \mathscr{T}(F, \mathscr{M})$.

Lemma 2.5.12. The assertions (a) and (b) are equivalent and imply (c) .

Proof. Assume for example that (b) is true. The pairing (2.5.12) induces a morphism

$$
\mathscr{W}(\mathscr{M}, F) \rightarrow \mathrm{D}_{X}(\mathscr{T}(F, \mathscr{M})) .
$$

For any relatively compact open subanalytic subset $U, \mathrm{R} \Gamma_{c}(U ; \mathscr{T}(F, \mathscr{M}))$ has finite-dimensional cohomologies by (b), and the morphism induced by $(2.5 .15)$

$\mathrm{R} \Gamma(U ; \mathscr{W}(\mathscr{M}, F)) \rightarrow \mathrm{R} \Gamma\left(U ; \mathrm{D}_{X}(\mathscr{T}(F, \mathscr{M}))\right) \simeq \operatorname{Hom}\left(\mathrm{R} \Gamma_{c}(U ; \mathscr{T}(F, \mathscr{M})), \mathbb{C}\right)$

is an isomorphism by Theorem 2.5.11. Hence (2.5.15) is an isomorphism, which implies (a) and (c). Q.E.D.

Theorem 2.5.13. Let $\mathscr{M} \in \mathrm{D}_{\text {hol }}^{\mathrm{b}}\left(\mathscr{D}_{X}\right)$ and $F \in \mathrm{D}_{\mathbb{R}-c}^{\mathrm{b}}\left(\mathbb{C}_{X}\right)$. Then assertions (a), (b), (c) in (2.5.14) hold true.

This result will be proved in Corollary 7.7.2 below. Note that it solves a conjecture in [KS03, Conjecture 6.2].

Applying this result in the situation of Example 2.5.9 (ii), we get:

Corollary 2.5.14. Let $M$ be a real analytic manifold, $X$ a complexification of $M$ and let $\mathscr{M} \in \mathrm{D}_{\mathrm{hol}}^{\mathrm{b}}\left(\mathscr{D}_{X}\right)$. Then the two objects $\mathrm{R} \mathscr{H}{ }_{0} m_{\mathscr{D}_{X}}\left(\mathscr{M}, \mathcal{C}_{M}^{\infty}\right)$ and $\mathcal{D} b_{M}^{\mathrm{tV}} \stackrel{\mathrm{L}}{\otimes}_{\mathscr{D}_{X}} \mathscr{M}$ belong to $\mathrm{D}_{\mathbb{R}-c}^{\mathrm{b}}\left(\mathbb{C}_{M}\right)$ and are dual to each other. Namely, we have $\mathrm{D}_{M} \mathrm{R} \mathscr{H}{ }_{0 m_{\mathscr{D}_{X}}}\left(\mathscr{M}, \mathcal{C}_{M}^{\infty}\right) \simeq \mathcal{D} b_{M}^{\mathrm{tv}} \stackrel{\mathrm{L}}{\mathscr{D}_{X}}, \mathscr{M}$. 
Corollary 2.5.15. Assume that $\mathscr{M} \in \mathrm{D}_{\text {hol }}^{\mathrm{b}}\left(\mathscr{D}_{X}\right), F \in \mathrm{D}_{\mathbb{R}_{-c}}^{\mathrm{b}}\left(\mathbb{C}_{X}\right)$ and $\operatorname{Supp}(F)$ is compact. Then the complexes

$\mathrm{R} \Gamma\left(X ; \mathrm{R} \mathscr{H} o m_{\mathscr{D}_{X}}\left(\mathscr{M}, F \stackrel{\mathrm{w}}{\otimes} \mathscr{O}_{X}\right)\right)$ and $\mathrm{R} \Gamma\left(X ; \mathrm{R} \mathscr{H} o m_{\mathrm{IC}_{X}}\left(F, \Omega_{X}^{\mathrm{t}}\left[d_{X}\right]\right) \stackrel{\mathrm{L}}{\otimes}_{\mathscr{D}_{X}} \mathscr{M}\right)$ have finite-dimensional cohomologies and (2.5.12) induces a perfect pairing for all $i \in \mathbb{Z}$

$$
H^{-i} \mathrm{R} \Gamma(X ; \mathscr{W}(\mathscr{M}, F)) \otimes H^{i} \mathrm{R} \Gamma(X ; \mathscr{T}(F, \mathscr{M})) \rightarrow \mathbb{C} .
$$

Remark 2.5.16. It follows immediately from [Ka 78, Ka 84] that (b), hence (a) and (c), are true when $F \in \mathrm{D}_{\mathbb{C}-\mathrm{c}}^{\mathrm{b}}\left(\mathbb{C}_{X}\right)$.

In [BE 04], S. Bloch and H. Esnault proved directly a similar result on an algebraic curve $X$ when assuming that $\mathscr{M}$ is a meromorphic connection with poles on a divisor $D$ and $F=\mathbb{C}_{X}$. They interpret the duality pairing by considering sections of the type $\gamma \otimes \epsilon$, where $\gamma$ is a cycle with boundary on $D$ and $\epsilon$ is a horizontal section of the connection on $\gamma$ with exponential decay on $D$. Their work has been extended to higher dimension by M. Hien [Hi 09].

\section{Tempered solutions of D-modules}

\subsection{Tempered de Rham and Sol functors}

Setting $\Omega_{X}^{\mathrm{t}}:=\Omega_{X} \otimes_{\mathscr{O}_{X}} \mathscr{O}_{X}^{\mathrm{t}}$, we define the tempered de Rham and solution functors by

$$
\begin{aligned}
\mathcal{D} \mathcal{R}_{X}^{\mathrm{t}}: \mathrm{D}^{\mathrm{b}}\left(\mathscr{D}_{X}\right) \rightarrow \mathrm{D}^{-}\left(\mathrm{IC}_{X}\right), & \mathscr{M} \mapsto \Omega_{X}^{\mathrm{t}} \stackrel{\mathrm{L}}{\mathscr{Q}_{\mathscr{D}_{X}}}, \mathscr{M}, \\
\mathcal{S}_{o l}^{\mathrm{t}}: \mathrm{D}^{\mathrm{b}}\left(\mathscr{D}_{X}\right)^{\mathrm{op}} \rightarrow \mathrm{D}^{+}\left(\mathrm{IC}_{X}\right), & \mathscr{M} \mapsto \mathrm{R} \mathscr{H}{ }^{\circ} m_{\mathscr{D}_{X}}\left(\mathscr{M}, \mathscr{O}_{X}^{\mathrm{t}}\right) .
\end{aligned}
$$

One has

$$
\mathcal{S}_{o} l_{X} \alpha_{X} \mathcal{S}_{X}^{\mathrm{t}}, \quad \mathcal{D} \mathcal{R}_{X} \simeq \alpha_{X} \mathcal{D} \mathcal{R}_{X}^{\mathrm{t}} .
$$

For $\mathscr{M} \in \mathrm{D}_{\text {coh }}^{\mathrm{b}}\left(\mathscr{D}_{X}\right)$, one has

$$
\operatorname{Sol}_{X}^{\mathrm{t}}(\mathscr{M}) \simeq \mathcal{D} \mathcal{R}_{X}^{\mathrm{t}}\left(\mathbb{D}_{X} \mathscr{M}\right)\left[-d_{X}\right] .
$$

The next result is a reformulation of a theorem of [Ka 84] (see also [KS 01, Th. 7.4.1]) 
Theorem 3.1.1. Let $f: X \rightarrow Y$ be a morphism of complex manifolds. There is an isomorphism in $\mathrm{D}^{\mathrm{b}}\left(\mathrm{I}^{-1} \mathscr{D}_{Y}^{\mathrm{op}}\right)$ :

$$
\Omega_{X}^{\mathrm{t}} \stackrel{\mathrm{L}}{\otimes}_{\mathscr{D}_{X}} \mathscr{D}_{X \rightarrow Y}\left[d_{X}\right] \stackrel{\sim}{\sim} f^{!} \Omega_{Y}^{\mathrm{t}}\left[d_{Y}\right] .
$$

Proof. Consider isomorphism (2.5.4) with $M=X_{\mathbb{R}}$ and $N=Y_{\mathbb{R}}$ and apply - $\stackrel{\mathrm{L}}{\otimes}_{\mathscr{D}_{Y^{c}}} \mathscr{O}_{Y^{c}}$. We get the result since

$$
\begin{aligned}
& \text { - } \stackrel{\mathrm{L}}{\otimes}_{\mathscr{D}_{X \times X^{c}}} \mathscr{D}_{X \times X^{c} \rightarrow Y \times Y^{c}} \stackrel{\mathrm{L}}{\otimes_{\mathscr{D}_{Y c}}} \mathscr{O}_{Y^{c}} \\
& \simeq \stackrel{\mathrm{L}}{\otimes}_{\mathscr{D}_{X \times X^{c}}} \mathscr{D}_{X \times X^{c} \rightarrow Y \times Y^{c}} \stackrel{\mathrm{L}}{\mathscr{D}_{Y^{c}}} \mathscr{D}_{Y^{c} \rightarrow \mathrm{pt}} \\
& \simeq \stackrel{\mathrm{L}}{\otimes}_{\mathscr{D}_{X \times X^{c}}} \mathscr{D}_{X \times X^{c} \rightarrow Y \times Y^{c}} \stackrel{\mathrm{L}}{\otimes}_{\mathscr{D}_{Y \times Y^{c}}} \mathscr{D}_{Y \times Y^{c} \rightarrow Y} \\
& \simeq \stackrel{\mathrm{L}}{\otimes}_{\mathscr{D}_{X \times X^{c}}} \mathscr{D}_{X \times X^{c} \rightarrow Y} \\
& \simeq \cdot \stackrel{\mathrm{L}}{\otimes}_{\mathscr{D}_{X}} \mathscr{D}_{X \rightarrow Y} \stackrel{\mathrm{L}}{\otimes}_{\mathscr{D}_{X^{c}}} \mathscr{O}_{X^{c}} \text {. }
\end{aligned}
$$

Q.E.D.

Note that this isomorphism (3.1.2) is equivalent to the isomorphism

$$
\mathscr{D}_{Y \leftarrow X} \stackrel{\mathrm{L}}{\otimes} \mathscr{D}_{X} \mathscr{O}_{X}^{\mathrm{t}}\left[d_{X}\right] \stackrel{\sim}{\longrightarrow} f^{!} \mathscr{O}_{Y}^{\mathrm{t}}\left[d_{Y}\right] \quad \text { in } \mathrm{D}^{\mathrm{b}}\left(\mathrm{I}^{-1} \mathscr{D}_{Y}\right) .
$$

Corollary 3.1.2. Let $f: X \rightarrow Y$ be a morphism of complex manifolds and let $\mathscr{N} \in \mathrm{D}^{\mathrm{b}}\left(\mathscr{D}_{Y}\right)$. Then (3.1.2) induces the isomorphism

$$
\mathcal{D} \mathcal{R}_{X}^{\mathrm{t}}\left(\mathrm{D} f^{*} \mathscr{N}\right)\left[d_{X}\right] \simeq f^{!} \mathcal{D} \mathcal{R}_{Y}^{\mathrm{t}}(\mathscr{N})\left[d_{Y}\right] \quad \text { in } \mathrm{D}^{\mathrm{b}}\left(\mathrm{I} \mathbb{C}_{X}\right) \text {. }
$$

Proof. Apply $\bullet \stackrel{\mathrm{L}}{\otimes}_{f^{-1} \mathscr{D}_{Y}} f^{-1} \mathscr{N}$ to isomorphism (3.1.2).

Q.E.D.

Corollary 3.1.3. For any complex manifold $X$, we have

$$
\mathcal{D} \mathcal{R}_{X}^{\mathrm{t}}\left(\mathscr{O}_{X}\right) \simeq \mathbb{C}_{X}\left[d_{X}\right] .
$$

Corollary 3.1.4. Let $f: X \rightarrow Y$ be a morphism of complex manifolds. There is a natural morphism

$$
f^{-1} \Omega_{Y}^{\mathrm{t}} \stackrel{\mathrm{L}}{\otimes}_{f^{-1} \mathscr{D}_{Y}} \mathscr{D}_{Y} \leftarrow X \longrightarrow \Omega_{X}^{\mathrm{t}} \text { in } \mathrm{D}^{\mathrm{b}}\left(\mathrm{I}_{X}^{\mathrm{op}}\right) .
$$


Proof. (i) Assume that $f$ is a closed embedding. We have

$$
\begin{aligned}
& f^{-1} \Omega_{Y}^{\mathrm{t}} \stackrel{\mathrm{L}}{\otimes}_{f^{-1} \mathscr{D}_{Y}} \mathscr{D}_{Y \leftarrow X} \simeq f^{!} \mathrm{R} f_{! !}\left(f^{-1} \Omega_{Y}^{\mathrm{t}} \stackrel{\mathrm{Q}}{\otimes}_{f^{-1} \mathscr{D}_{Y}} \mathscr{D}_{Y \leftarrow X}\right) \\
& \simeq f^{!}\left(\Omega_{Y}^{\mathrm{t}} \stackrel{\mathrm{L}}{\otimes}_{\mathscr{D}_{Y}} \mathrm{R} f_{!} \mathscr{D}_{Y \leftarrow X}\right) \\
& \simeq f^{!} \Omega_{Y}^{\mathrm{t}} \stackrel{\mathrm{L}}{\otimes}_{f^{-1} \mathscr{D}_{Y}} f^{-1} \mathscr{D}_{Y \leftarrow X} \\
& \simeq \Omega_{X}^{\mathrm{t}} \stackrel{\mathrm{L}}{\otimes}_{\mathscr{D}_{X}} \mathscr{D}_{X \rightarrow Y} \stackrel{\mathrm{L}}{\otimes}_{f^{-1} \mathscr{D}_{Y}} \mathscr{D}_{Y \leftarrow X}\left[d_{X}-d_{Y}\right] \\
& \simeq \Omega_{X}^{\mathrm{t}} \text {. }
\end{aligned}
$$

(ii) Assume that $f$ is submersive. We have

$$
\begin{aligned}
\operatorname{R} \mathscr{H} o m_{\mathscr{D}_{X}^{\text {op }}}\left(\mathscr{D}_{Y \leftarrow X}, \Omega_{X}^{\mathrm{t}}\right) & \simeq \Omega_{X}^{\mathrm{t}} \stackrel{\mathrm{L}}{\otimes_{\mathscr{D}_{X}}} \operatorname{R} \mathscr{H}_{o m_{X}^{\text {op }}}\left(\mathscr{D}_{Y \leftarrow X}, \mathscr{D}_{X}\right) \\
& \simeq \Omega_{X}^{\mathrm{t}} \stackrel{\mathrm{L}}{\otimes}_{\mathscr{D}_{X}} \mathscr{D}_{X} \rightarrow_{Y}\left[d_{Y}-d_{X}\right] \\
& \simeq f^{!} \Omega_{Y}^{\mathrm{t}}\left[2 d_{Y}-2 d_{X}\right] \simeq f^{-1} \Omega_{Y}^{\mathrm{t}} .
\end{aligned}
$$

Then use

$$
\mathrm{R} \mathscr{H}_{o m_{X}^{\text {op }}}\left(\mathscr{D}_{Y \leftarrow X}, \Omega_{X}^{\mathrm{t}}\right){\stackrel{\mathrm{L}}{f^{-1}}}_{\mathscr{D}_{Y}} \mathscr{D}_{Y \leftarrow X} \rightarrow \Omega_{X}^{\mathrm{t}} .
$$

Q.E.D.

Note that morphism (3.1.5) is equivalent to the morphism in $\mathrm{D}^{\mathrm{b}}\left(\mathrm{I} \mathscr{D}_{X}\right)$

$$
\mathscr{D}_{X \rightarrow Y} \stackrel{\mathrm{L}}{\otimes}_{f^{-1} \mathscr{D}_{Y}} f^{-1} \mathscr{O}_{Y}^{\mathrm{t}} \longrightarrow \mathscr{O}_{X}^{\mathrm{t}} .
$$

The next result is a kind of Grauert direct image theorem for tempered holomorphic functions. It will be generalised to D-modules in Corollary 3.1.6. Its proof uses difficult results of functional analysis.

Theorem 3.1.5 (Tempered Grauert theorem [KS96, Th. 7.3]). Let $f: X \rightarrow$ $Y$ be a morphism of complex manifolds, let $\mathscr{F} \in \mathrm{D}_{\text {coh }}^{\mathrm{b}}\left(\mathscr{O}_{X}\right)$ and assume that $f$ is proper on $\operatorname{Supp}(\mathscr{F})$. Then there is a natural isomorphism

$$
\mathrm{R} f_{! !}\left(\mathscr{O}_{X}^{\mathrm{t}} \stackrel{\mathrm{L}}{\otimes}_{\mathscr{O}_{X}} \mathscr{F}\right) \simeq \mathscr{O}_{Y}^{\mathrm{t}} \stackrel{\mathrm{L}}{\otimes}_{\mathscr{O}_{Y}} \mathrm{R} f_{!} \mathscr{F} .
$$


An indication on the proof. It is enough to prove that for any $G \in \mathbb{R}-\mathrm{C}\left(\mathbb{C}_{Y}\right)$, we have

$$
\mathrm{R} \mathscr{H} o m\left(f^{-1} G, \mathscr{O}_{X}^{\mathrm{t}} \stackrel{\mathrm{L}}{\otimes}_{\mathscr{O}_{X}} \mathscr{F}\right) \simeq \mathrm{R} \mathscr{H} o m\left(G, \mathscr{O}_{Y}^{\mathrm{t}} \stackrel{\mathrm{L}}{\otimes}_{\mathscr{O}_{Y}} \mathrm{R} f ! \mathscr{F}\right) .
$$

Since $\mathscr{F}$ and $\mathrm{R} f_{!} \mathscr{F}$ are coherent, (3.1.7) is equivalent to

$$
\mathrm{R} \mathscr{H} o m\left(f^{-1} G, \mathscr{O}_{X}^{\mathrm{t}}\right) \stackrel{\mathrm{L}}{\otimes}_{\mathscr{O}_{X}} \mathscr{F} \simeq \mathrm{R} \mathscr{H} o m\left(G, \mathscr{O}_{Y}^{\mathrm{t}}\right) \stackrel{\mathrm{L}}{\otimes}_{\mathscr{O}_{Y}} \mathrm{R} f_{!} \mathscr{F} .
$$

Such a formula is proved in [KS96, Th. 7.3].

Q.E.D.

Corollary 3.1.6 ([KS01, Th. 7.4.6]). Let $f: X \rightarrow Y$ be a morphism of complex manifolds. Let $\mathscr{M} \in \mathrm{D}_{\mathrm{q} \text {-good }}^{\mathrm{b}}\left(\mathscr{D}_{X}\right)$ and assume that $f$ is proper on $\operatorname{Supp}(\mathscr{M})$. Then there is an isomorphism in $\mathrm{D}^{\mathrm{b}}\left(\mathrm{IC}_{Y}\right)$

$$
\mathcal{D} \mathcal{R}_{Y}^{\mathrm{t}}\left(\mathrm{D} f_{*} \mathscr{M}\right) \stackrel{\sim}{\longrightarrow} f_{*} \mathcal{D} \mathcal{R}_{X}^{\mathrm{t}}(\mathscr{M}) .
$$

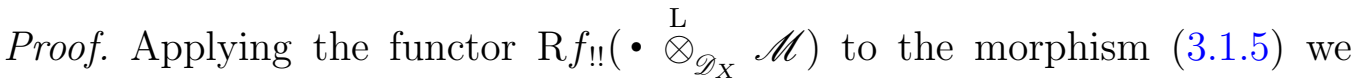
obtain the morphism in (3.1.9). To check it is an isomorphism, we reduce to the case where $\mathscr{M}=\mathscr{D}_{X} \otimes_{\mathscr{O}_{X}} \mathscr{F}$ with a coherent $\mathscr{O}_{X}$-module $\mathscr{F}$ such that $f$ is proper on $\operatorname{Supp}(\mathscr{F})$. Then we apply Theorem 3.1.5.

Q.E.D.

Corollary 3.1.7. Let $f$ and $\mathscr{M}$ be as in Corollary 3.1.6. Then we have the isomorphism

$$
\mathrm{D} f_{*}\left(\mathscr{O}_{X}^{\mathrm{t}} \stackrel{\mathrm{D}}{\otimes} \mathscr{M}\right) \simeq \mathscr{O}_{Y}^{\mathrm{t}} \stackrel{\mathrm{D}}{\otimes} \mathrm{D} f_{*} \mathscr{M} \text { in } \mathrm{D}^{\mathrm{b}}\left(\mathrm{I} \mathscr{D}_{Y}\right)
$$

Proof. We have

$$
\begin{aligned}
& \Omega_{Y}^{\mathrm{t}} \stackrel{\mathrm{D}}{\otimes} \mathrm{D} f_{!} \mathscr{M} \simeq \mathscr{O}_{Y}^{\mathrm{t}} \stackrel{\mathrm{L}}{\otimes} \mathscr{D}_{Y}\left(\mathscr{D}_{Y} \stackrel{\mathrm{D}}{\otimes} \mathrm{D} f_{!} \mathscr{M}\right) \\
& \simeq \mathscr{O}_{Y}^{\mathrm{t}} \stackrel{\mathrm{L}}{\otimes} \mathscr{D}_{Y} \mathrm{D} f_{!}\left(\mathrm{D} f^{*} \mathscr{D}_{Y} \stackrel{\mathrm{D}}{\otimes} \mathscr{M}\right) \\
& \simeq \mathcal{D} \mathcal{R}_{Y}^{\mathrm{t}}\left(\mathrm{D} f_{!}\left(\mathscr{D}_{X \rightarrow Y} \stackrel{\mathrm{D}}{\otimes} \mathscr{M}\right)\right) \text {, }
\end{aligned}
$$

where the second isomorphism follows from the projection formula (1.2.3). Applying Corollary 3.1.6, we obtain

$$
\Omega_{Y}^{\mathrm{t}} \stackrel{\mathrm{D}}{\otimes} \mathrm{D} f_{!} \mathscr{M} \simeq \operatorname{R} f_{*}\left(\Omega_{X}^{\mathrm{t}} \stackrel{\mathrm{L}}{\mathscr{D}_{X}}\left(\mathscr{D}_{X \rightarrow Y} \stackrel{\mathrm{D}}{\otimes} \mathscr{M}\right)\right) .
$$


On the other-hand, we have

$$
\Omega_{X}^{\mathrm{t}} \stackrel{\mathrm{L}}{\otimes_{\mathscr{D}_{X}}}\left(\mathscr{D}_{X} \rightarrow Y \stackrel{\mathrm{D}}{\otimes} \mathscr{M}\right) \simeq\left(\Omega_{X}^{\mathrm{t}} \stackrel{\mathrm{D}}{\otimes} \mathscr{M}\right) \stackrel{\mathrm{L}}{\otimes_{\mathscr{D}_{X}}} \mathscr{D}_{X} \rightarrow Y
$$

Therefore,

$$
\Omega_{Y}^{\mathrm{t}} \stackrel{\mathrm{D}}{\otimes} \mathrm{D} f_{!} \mathscr{M} \simeq \mathrm{D} f_{*}\left(\Omega_{X}^{\mathrm{t}} \stackrel{\mathrm{D}}{\otimes} \mathscr{M}\right)
$$

To conclude, use the equivalence of categories $\mathrm{D}^{\mathrm{b}}\left(\mathscr{D}_{Y}^{\mathrm{op}}\right) \simeq \mathrm{D}^{\mathrm{b}}\left(\mathscr{D}_{Y}\right)$ given by $\mathscr{M}^{\mathrm{r}}=\Omega_{X} \stackrel{\mathrm{L}}{\otimes}_{\mathscr{O}_{X}} \mathscr{M}$.

Q.E.D.

Remark 3.1.8. If one replaces (3.1.2) with its non-tempered version, then the formula is no more true, contrarily to isomorphism (3.1.9) which remains true by Theorem 1.2.1.

\subsection{Localization along a hypersurface}

In order to prove Theorem 4.3.2 below, a generalized form of the RiemannHilbert correspondence for regular holonomic D-modules, we need some lemmas.

If $S \subset X$ is a closed hypersurface, denote by $\mathscr{O}_{X}(* S)$ the sheaf of meromorphic functions with poles at $S$. It is a regular holonomic $\mathscr{D}_{X}$-module (see Definition 4.1 .1 below) and it is a flat $\mathscr{O}_{X}$-module. For $\mathscr{M} \in \mathrm{D}^{\mathrm{b}}\left(\mathrm{I} \mathscr{D}_{X}\right)$, set

$$
\mathscr{M}(* S)=\mathscr{M} \stackrel{\mathrm{D}}{\otimes} \mathscr{O}_{X}(* S)
$$

Lemma 3.2.1. Let $S$ be a closed complex hypersurface in $X$. There are isomorphisms

$$
\begin{aligned}
& \mathscr{O}_{X}^{\mathrm{t}}(* S) \simeq \mathrm{R} \mathscr{I h o m}\left(\mathbb{C}_{X \backslash S}, \mathscr{O}_{X}^{\mathrm{t}}\right) \text { in } \mathrm{D}^{\mathrm{b}}\left(\mathrm{I} \mathscr{D}_{X}\right) \\
& \mathscr{O}_{X}(* S) \simeq \mathrm{R} \mathscr{H} \text { om }_{\mathrm{IC}_{X}}\left(\mathbb{C}_{X \backslash S}, \mathscr{O}_{X}^{\mathrm{t}}\right) \text { in } \mathrm{D}^{\mathrm{b}}\left(\mathscr{D}_{X}\right) .
\end{aligned}
$$

Proof. (i) The second isomorphism follows from the first one by applying the functor $\alpha_{X}$.

(ii) By taking the Dolbeault resolution of $\mathscr{O}_{X}^{\mathrm{t}}$ we are reduced to prove a similar result with $\mathcal{D} b_{X_{\mathbb{R}}}^{\mathrm{t}}$ instead of $\mathscr{O}_{X}^{\mathrm{t}}$. More precisely, consider a real analytic manifold $M$, a real analytic map $f: M \rightarrow \mathbb{C}$. Set $S=\{f=0\}$ and denote by $j:(M \backslash S) \hookrightarrow M$ the open embedding. Define the sheaf $\mathscr{A}_{M}[1 / f]$ as the 
inductive limit of the sequence of embeddings $\mathscr{A}_{M} \stackrel{f}{\longrightarrow} \mathscr{A}_{M} \stackrel{f}{\longrightarrow} \cdots$. Equivalently, $\mathscr{A}_{M}[1 / f]$ is the subsheaf of $j_{*} j^{-1} \mathscr{A}_{M}$ consisting of sections $u$ such that there locally exists an integer $m$ with $f^{m} \cdot u \in \mathscr{A}_{M}$. Set

$$
\mathcal{D} b_{M}^{\mathrm{t}}[1 / f]:=\mathcal{D} b_{M}^{\mathrm{t}} \otimes_{\mathscr{A}_{M}}\left(\mathscr{A}_{M}[1 / f]\right) .
$$

(Note that $\mathcal{D} b_{M}^{\mathrm{t}}[1 / f]$ is isomorphic to the inductive limit of the sequence of morphisms $\mathcal{D} b_{M}^{\mathrm{t}} \stackrel{f}{\longrightarrow} \mathcal{D} b_{M}^{\mathrm{t}} \stackrel{f}{\longrightarrow} \cdots$.) It is enough to prove the isomorphism

$$
\mathcal{D} b_{M}^{\mathrm{t}}[1 / f] \simeq \mathrm{R} \mathscr{I h o m}\left(\mathbb{C}_{M \backslash S}, \mathcal{D} b_{M}^{\mathrm{t}}\right),
$$

or, equivalently, the isomorphism for any open relatively compact subanalytic subset $U$ of $M$

$$
\Gamma\left(U ; \mathcal{D} b_{M_{\mathrm{sa}}}^{\mathrm{t}}[1 / f]\right) \simeq \Gamma\left(U \backslash S ; \mathcal{D} b_{M_{\mathrm{sa}}}^{\mathrm{t}}\right) .
$$

This follows from the fact that $f: \Gamma\left(U \backslash S ; \mathcal{D} b_{M_{\mathrm{sa}}}^{\mathrm{t}}\right) \rightarrow \Gamma\left(U \backslash S ; \mathcal{D} b_{M_{\mathrm{sa}}}^{\mathrm{t}}\right)$ is bijective. (See also Lojasiewicz [Lo 59].) Q.E.D.

In the sequel, we set for a closed complex analytic hypersurface $S$

$$
\mathscr{O}_{X}^{\mathrm{t}}(* S):=\mathscr{O}_{X}^{\mathrm{t}} \stackrel{\mathrm{D}}{\otimes} \mathscr{O}_{X}(* S) \simeq \mathrm{R} \mathscr{I h o m}\left(\mathbb{C}_{M \backslash S}, \mathscr{O}_{X}^{\mathrm{t}}\right) .
$$

Lemma 3.2.2. Let $S$ be a closed complex hypersurface in $X$. There are isomorphisms

$$
\Omega_{X} \stackrel{\mathrm{L}}{\mathscr{Q}_{X}} \mathscr{O}_{X}^{\mathrm{t}}(* S) \stackrel{\sim}{\longrightarrow} \Omega_{X} \stackrel{\mathrm{L}}{\mathscr{Q}_{\mathscr{D}}} \mathscr{O}_{X}(* S) \simeq \mathrm{R} \mathscr{H} o m\left(\mathbb{C}_{X \backslash S}, \mathbb{C}_{X}\right)\left[d_{X}\right] .
$$

Proof. It follows from Lemma 3.2.1 that

$$
\Omega_{X} \stackrel{\mathrm{L}}{\mathscr{D}_{X}} \mathscr{O}_{X}^{\mathrm{t}}(* S) \simeq \mathrm{R} \mathscr{I h o m}\left(\mathbb{C}_{X \backslash S}, \Omega_{X} \stackrel{\mathrm{L}}{\otimes}_{\mathscr{D}_{X}} \mathscr{O}_{X}^{\mathrm{t}}\right) .
$$

Then the result follows from the isomorphisms

$$
\Omega_{X} \stackrel{\mathrm{L}}{\otimes_{\mathscr{D}_{X}}} \mathscr{O}_{X}^{\mathrm{t}} \simeq \Omega_{X} \stackrel{\mathrm{L}}{\mathscr{D}_{X}} \mathscr{O}_{X} \simeq \mathbb{C}_{X}\left[d_{X}\right]
$$

Q.E.D. 


\section{Regular holonomic D-modules}

\subsection{Regular normal form for holonomic modules}

For the notion of regular holonomic D-modules, refer e.g. to [Ka 03, §5.2] and [KK 81].

Definition 4.1.1. Let $\mathscr{M}$ be a holonomic $\mathscr{D}_{X}$-module, $\Lambda$ its characteristic variety in $T^{*} X$ and $\mathscr{I}_{\Lambda}$ the ideal of $\operatorname{gr}\left(\mathscr{D}_{X}\right)$ of functions vanishing on $\Lambda$. We say that $\mathscr{M}$ is regular if there exists locally a good filtration on $\mathscr{M}$ such that $\mathscr{I}_{\Lambda} \cdot \operatorname{gr}(\mathscr{M})=0$.

One can prove that the full subcategory $\operatorname{Mod}_{\mathrm{rh}}\left(\mathscr{D}_{X}\right)$ of $\operatorname{Mod}_{\text {coh }}\left(\mathscr{D}_{X}\right)$ consisting of regular holonomic $\mathscr{D}_{X}$-modules is a thick abelian subcategory, stable by duality. Denote by $\mathrm{D}_{\mathrm{rh}}^{\mathrm{b}}\left(\mathscr{D}_{X}\right)$ the full subcategory of $\mathrm{D}^{\mathrm{b}}\left(\mathscr{D}_{X}\right)$ whose objects have regular holonomic cohomologies. Then $\mathrm{D}_{\mathrm{rh}}^{\mathrm{b}}\left(\mathscr{D}_{X}\right)$ is triangulated.

For a coherent $\mathscr{D}_{X}$-module $\mathscr{M}$, denote by $\operatorname{SingSupp}(\mathscr{M})$ the set of $x \in X$ such that $\mathscr{M}$ is not a coherent $\mathscr{O}_{X}$-module on a neighborhood of $x$.

Definition 4.1.2. Let $X$ be a complex manifold and $D \subset X$ a normal crossing divisor. We say that a holonomic $\mathscr{D}_{X}$-module $\mathscr{M}$ has regular normal form along $D$ if locally on $D$, for a local coordinate system $\left(z_{1}, \ldots, z_{n}\right)$ on $X$ such that $D=\left\{z_{1} \cdots z_{r}=0\right\}, \mathscr{M} \simeq \mathscr{D}_{X} / \mathscr{I}_{\lambda}$ for $\lambda=\left(\lambda_{1}, \ldots, \lambda_{r}\right) \in\left(\mathbb{C} \backslash \mathbb{Z}_{\geq_{0}}\right)^{r}$. Here, $\mathscr{I}_{\lambda}$ is the left ideal generated by the operators $\left(z_{i} \partial_{i}-\lambda_{i}\right)$ and $\partial_{j}$ for $i \in\{1, \ldots, r\}, j \in\{r+1, \ldots, n\}$.

One shall be aware that the property of being of normal form is not stable by duality. Note that, for $\lambda=\left(\lambda_{1}, \ldots, \lambda_{r}\right) \in \mathbb{C}^{m}, \mathscr{D}_{X} / \mathscr{I}_{\lambda} \stackrel{\sim}{\longrightarrow}\left(\mathscr{D}_{X} / \mathscr{I}_{\lambda}\right)(* D)$ if and only if $\lambda_{i} \in \mathbb{C} \backslash \mathbb{Z}_{\geq 0}$ for any $i \in\{1, \ldots, r\}$.

Of course, if a holonomic $\mathscr{D}_{X}$-module has regular normal form, then it is regular holonomic.

Lemma 4.1.3. Let $\mathscr{L}$ be a holonomic module with regular normal form along $D$. Then we have the natural isomorphism $\mathcal{S}_{X}(\mathscr{L}) \otimes \mathbb{C}_{X \backslash D} \sim \mathcal{S}_{\text {ol }}(\mathscr{L})$.

Proof. It is enough to prove that $\left.\mathcal{S}_{X}(\mathscr{L})\right|_{D} \simeq 0$. In a local coordinate system $\left(z_{1}, \ldots, z_{n}\right)$ as in Definition 4.1.2, set $Z_{i}=\left\{z_{i}=0\right\}$. Setting $P_{i}=z_{i} \partial_{i}-\lambda_{i}$ with $\lambda_{i} \in \mathbb{C} \backslash \mathbb{Z}_{\geq 0}$, it is enough to check that $P_{i}$ induces an isomorphism $P_{i}:\left.\left.\mathscr{O}_{X}\right|_{Z_{i}} \stackrel{\sim}{\longrightarrow} \mathscr{O}_{X}\right|_{Z_{i}}$, which is clear.

Q.E.D. 
Lemma 4.1.4. Let $P_{X}(\mathscr{M})$ be a statement concerning a complex manifold $X$ and a regular holonomic object $\mathscr{M} \in \mathrm{D}_{\mathrm{rh}}^{\mathrm{b}}\left(\mathscr{D}_{X}\right)$. Consider the following conditions.

(a) Let $X=\bigcup_{i \in I} U_{i}$ be an open covering. Then $P_{X}(\mathscr{M})$ is true if and only if $P_{U_{i}}\left(\left.\mathscr{M}\right|_{U_{i}}\right)$ is true for any $i \in I$.

(b) If $P_{X}(\mathscr{M})$ is true, then $P_{X}(\mathscr{M}[n])$ is true for any $n \in \mathbb{Z}$.

(c) Let $\mathscr{M}^{\prime} \rightarrow \mathscr{M} \rightarrow \mathscr{M}^{\prime \prime} \stackrel{+1}{\longrightarrow}$ be a distinguished triangle in $\mathrm{D}_{\mathrm{rh}}^{\mathrm{b}}\left(\mathscr{D}_{X}\right)$. If $P_{X}\left(\mathscr{M}^{\prime}\right)$ and $P_{X}\left(\mathscr{M}^{\prime \prime}\right)$ are true, then $P_{X}(\mathscr{M})$ is true.

(d) Let $\mathscr{M}$ and $\mathscr{M}^{\prime}$ be regular holonomic $\mathscr{D}_{X}$-modules. If $P_{X}\left(\mathscr{M} \oplus \mathscr{M}^{\prime}\right)$ is true, then $P_{X}(\mathscr{M})$ is true.

(e) Let $f: X \rightarrow Y$ be a projective morphism and let $\mathscr{M}$ be a good regular holonomic $\mathscr{D}_{X}$-module. If $P_{X}(\mathscr{M})$ is true, then $P_{Y}\left(\mathrm{D} f_{*} \mathscr{M}\right)$ is true.

(f) If $\mathscr{M}$ is a regular holonomic $\mathscr{D}_{X}$-module with a regular normal form along a normal crossing divisor of $X$, then $P_{X}(\mathscr{M})$ is true.

If conditions (a)-(f) are satisfied, then $P_{X}(\mathscr{M})$ is true for any complex manifold $X$ and any $\mathscr{M} \in \mathrm{D}_{\mathrm{rh}}^{\mathrm{b}}\left(\mathscr{D}_{X}\right)$.

Sketch of proof. (i) If $D$ is a normal crossing hypersurface of $X$ and $\mathscr{M}$ is a regular holonomic $\mathscr{D}_{X}$-module satisfying

- $\mathscr{M} \simeq \mathscr{M}(* D)$,

- $\operatorname{SingSupp}(\mathscr{M}) \subset D$,

then, locally on $X$, there exists a filtration

$$
\mathscr{M}=\mathscr{M}_{0} \supset \mathscr{M}_{1} \supset \cdots \supset \mathscr{M}_{l} \supset \mathscr{M}_{l+1}=0
$$

such that $\mathscr{M}_{j} / \mathscr{M}_{j+1}$ has regular normal form. It follows that in this case, $P_{X}(\mathscr{M})$ is true.

(ii) Let us take a closed complex analytic subset $Z$ of $X$ such that the support of $\mathscr{M}$ is contained in $Z$. We argue by induction on the dimension $m$ of $Z$. There exists a morphism $f: W \rightarrow Z$ such that

(1) $W$ is non singular with dimension $m$,

(2) $f$ is projective,

(3) there exists a closed complex analytic subset $S$ of $Z$ with dimension $<m$ 
such that

- $f^{-1}(Z \backslash S) \rightarrow Z \backslash S$ is an isomorphism,

- $D:=f^{-1} S$ is a normal crossing hypersurface of $W$,

- SingSupp $\left(H^{m-d_{X}} \mathrm{D} g^{*} \mathscr{M}\right) \subset D$, where $g$ is the composition $W \stackrel{f}{\longrightarrow} Z \hookrightarrow$ $X$.

We have

$$
\left(\mathrm{D} g^{*} \mathscr{M}\right)(* D) \simeq\left(\left(H^{m-d_{X}} \mathrm{D} g^{*} \mathscr{M}\right)(* D)\right)\left[d_{X}-m\right] .
$$

Then by step (i), $P_{W}\left(\left(\mathrm{D} g^{*} \mathscr{M}\right)(* D)\right)$ is true. Hence $P_{X}\left(\mathrm{D} g_{*}\left(\left(\mathrm{D} g^{*} \mathscr{M}\right)(* D)\right)\right)$ is true. Let us consider a distinguished triangle

$$
\mathscr{M} \longrightarrow \mathrm{D} g_{*}\left(\left(\mathrm{D} g^{*} \mathscr{M}\right)(* D)\right)\left[m-d_{X}\right] \longrightarrow \mathscr{N} \stackrel{1}{\longrightarrow} .
$$

Since $\operatorname{Supp}(\mathscr{N}) \subset S, P_{X}(\mathscr{N})$ is true by the induction hypothesis. Hence $P_{X}(\mathscr{M})$ is true. $\quad$ Q.E.D.

Remark 4.1.5. In fact, we could remove condition (d) in the regular case. We keep it by analogy with the irregular case (Lemma 4.1.4).

\subsection{Real blow up}

A classical tool in the study of differential equations is the real blow up, and we shall use this construction in the proof of Theorems 4.3.2, 7.8.1 and in the definition of normal form given in $\S 7.5$.

Recall that $\mathbb{C}^{\times}$denotes $\mathbb{C} \backslash\{0\}$ and $\mathbb{R}_{>0}$ the multiplicative group of positive real numbers. Consider the action of $\mathbb{R}_{>0}$ on $\mathbb{C}^{\times} \times \mathbb{R}$ :

$$
\mathbb{R}_{>0} \times\left(\mathbb{C}^{\times} \times \mathbb{R}\right) \rightarrow \mathbb{C}^{\times} \times \mathbb{R}, \quad(a,(z, t)) \mapsto\left(a z, a^{-1} t\right)
$$

and set

$$
\widetilde{\mathbb{C}}^{\text {tot }}=\left(\mathbb{C}^{\times} \times \mathbb{R}\right) / \mathbb{R}_{>0}, \widetilde{\mathbb{C}}^{\geq 0}=\left(\mathbb{C}^{\times} \times \mathbb{R}_{\geq 0}\right) / \mathbb{R}_{>0}, \widetilde{\mathbb{C}}^{>0}=\left(\mathbb{C}^{\times} \times \mathbb{R}_{>0}\right) / \mathbb{R}_{>0} .
$$

One denotes by $\varpi^{\text {tot }}$ the map:

$$
\varpi^{\text {tot }}: \widetilde{\mathbb{C}}^{\text {tot }} \rightarrow \mathbb{C}, \quad(z, t) \mapsto t z .
$$

Then we have

$$
\widetilde{\mathbb{C}}^{\text {tot }} \supset \widetilde{\mathbb{C}}^{\geq 0} \supset \widetilde{\mathbb{C}}^{>0} \stackrel{\sim}{\longrightarrow} \mathbb{C}^{\times}
$$


Let $X=\mathbb{C}^{n} \simeq \mathbb{C}^{r} \times \mathbb{C}^{n-r}$ and let $D$ be the divisor $\left\{z_{1} \cdots z_{r}=0\right\}$. Set

$$
\widetilde{X}^{\text {tot }}=\left(\widetilde{\mathbb{C}}^{\text {tot }}\right)^{r} \times \mathbb{C}^{n-r}, \widetilde{X}^{>0}=\left(\widetilde{\mathbb{C}}^{>0}\right)^{r} \times \mathbb{C}^{n-r}, \widetilde{X}=\left(\widetilde{\mathbb{C}}^{\geq 0}\right)^{r} \times \mathbb{C}^{n-r} .
$$

Then $\widetilde{X}$ is the closure of $\widetilde{X}^{>0}$ in $\tilde{X}^{\text {tot }}$. The map $\varpi^{\text {tot }}$ in (4.2.2) defines the map

$$
\varpi: \widetilde{X} \rightarrow X \text {. }
$$

The map $\varpi$ is proper and induces an isomorphism

$$
\left.\varpi\right|_{\tilde{X}>0}: \tilde{X}^{>0}=\varpi^{-1}(X \backslash D) \stackrel{\sim}{\longrightarrow} \backslash \backslash D .
$$

We call $\widetilde{X}$ the real blow up along $D$.

Remark 4.2.1. The real manifold $\widetilde{X}$ (with boundary) as well as the map $\varpi: \widetilde{X} \rightarrow X$ may be intrinsically defined for a complex manifold $X$ and a normal crossing divisor $D$, but $\tilde{X}^{\text {tot }}$ is only intrinsically defined as a germ of a manifold in a neighborhood of $\widetilde{X}$.

We set

$$
\begin{aligned}
\mathcal{D} b_{\tilde{X}}^{\mathrm{t}} & :=\left.\mathscr{I h o m}\left(\mathbb{C}_{\tilde{X}>0}, \mathcal{D} b_{\tilde{X}_{\text {tot }}^{\mathrm{t}}}^{\mathrm{t}}\right)\right|_{\tilde{X}} \\
& \simeq \varpi^{!} \mathscr{I h o m}\left(\mathbb{C}_{X \backslash D}, \mathcal{D} b_{X_{\mathbb{R}}}^{\mathrm{t}}\right),
\end{aligned}
$$

where the last isomorphism follows from Lemma 2.5.7. Note that $\mathcal{D} b_{\tilde{X}}^{\mathrm{t}}$ is an object of $\mathrm{I}\left(\varpi^{-1} \mathscr{D}_{X} \otimes \varpi^{-1} \mathscr{D}_{X^{c}}\right)$.

Now we set

$$
\begin{aligned}
\mathscr{O}_{\tilde{X}}^{\mathrm{t}} & :=\mathrm{R} \mathscr{H} O m_{\varpi^{-1} \mathscr{D}_{X c}}\left(\varpi^{-1} \mathscr{O}_{X^{c}}, \mathcal{D} b_{\tilde{X}}^{\mathrm{t}}\right), \\
\mathcal{A}_{\tilde{X}} & :=\alpha_{\tilde{X}} \mathscr{O}_{\tilde{X}}^{\mathrm{t}}, \\
\mathscr{D}_{\tilde{X}}^{\mathcal{A}} & :=\mathcal{A}_{\tilde{X}} \otimes_{\varpi^{-1} \mathscr{O}_{X}} \varpi^{-1} \mathscr{D}_{X} .
\end{aligned}
$$

Then $\mathcal{A}_{\tilde{X}}$ and $\mathscr{D}_{\tilde{X}}^{\mathcal{A}}$ are concentrated in degree 0 , and hence they are sheaves of $\mathbb{C}$-algebras on $\widetilde{X}$. Indeed, $\mathcal{A}_{\widetilde{X}}$ is the subsheaf of $j_{*} j^{-1} \varpi^{-1} \mathscr{O}_{X}$ consisting of holomorphic functions tempered at any point of $\widetilde{X} \backslash \widetilde{X}^{>0}=\varpi^{-1}(D)$. Here, $j: \widetilde{X}>0 \hookrightarrow \widetilde{X}$ is the inclusion. Clearly, $\mathcal{D} b_{\widetilde{X}}^{t}$ is an object of $\mathrm{I}\left(\mathscr{D} \mathcal{A} \otimes \varpi^{-1} \mathscr{D}_{X^{c}}\right)$, and hence

$$
\mathscr{O}_{\widetilde{X}}^{\mathrm{t}} \text { is an object of } \mathrm{D}^{\mathrm{b}}\left(\mathrm{I}_{\mathscr{D}}^{\mathcal{A}}\right) \text {. }
$$


By using (4.2.3), we get the isomorphism

$$
\mathscr{O}_{\widetilde{X}}^{\mathrm{t}} \simeq \varpi^{!} \mathscr{O}_{X}^{\mathrm{t}}(* D) \text { in } \mathrm{D}^{\mathrm{b}}\left(\mathrm{I}^{-1} \mathscr{D}_{X}\right)
$$

Recall that the map $\varpi$ is proper, and hence $R \varpi ! ! ~ R \varpi_{*}$.

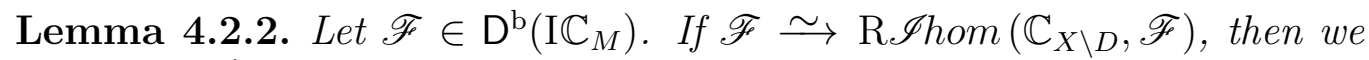
have $\mathrm{R} \varpi ! ! \varpi ! \mathscr{F} \stackrel{\mathscr{F}}{\sim}$.

Proof. One has

$$
\begin{aligned}
\mathrm{R} \varpi_{! !} ! \mathscr{F} & \simeq \mathrm{R} \varpi_{*} \varpi^{!} \operatorname{R} \mathscr{I h o m}\left(\mathbb{C}_{X \backslash D}, \mathscr{F}\right) \\
& \simeq \mathrm{R} \varpi_{*} \mathrm{R} \mathscr{I h o m}\left(\varpi^{-1} \mathbb{C}_{X \backslash D}, \varpi^{!} \mathscr{F}\right) \\
& \simeq \mathrm{R} \mathscr{I h o m}\left(\mathrm{R} \varpi_{! !} \varpi^{-1} \mathbb{C}_{X \backslash D}, \mathscr{F}\right) \simeq \mathscr{F} .
\end{aligned}
$$

Q.E.D.

As a corollary, we obtain the isomorphism

$$
\mathrm{R} \varpi_{*} \mathscr{O}_{\widetilde{X}}^{\mathrm{t}} \simeq \mathscr{O}_{X}^{\mathrm{t}}(* D) \text { in } \mathrm{D}^{\mathrm{b}}\left(\mathrm{I} \mathscr{D}_{X}\right)
$$

For $\mathscr{N} \in \mathrm{D}^{\mathrm{b}}\left(\mathscr{D}_{\widetilde{X}}^{\mathcal{A}}\right)$, we set

$$
\begin{aligned}
& \mathcal{D} \mathcal{R}_{\widetilde{X}}^{\mathrm{t}}(\mathscr{N})=\Omega_{\widetilde{X}}^{\mathrm{t}} \stackrel{\mathrm{L}}{\otimes_{\mathscr{D} \mathcal{A}}} \mathscr{N}, \\
& \mathcal{S}_{0} l_{\widetilde{X}}^{\mathrm{t}}(\mathscr{N})=\operatorname{R} \mathscr{H}_{0 m_{\mathscr{D}}^{\mathcal{A}}}\left(\mathscr{N}, \mathscr{O}_{\widetilde{X}}^{\mathrm{t}}\right) \text {. }
\end{aligned}
$$

Here $\Omega_{\widetilde{X}}^{\mathrm{t}}:=\varpi^{-1} \Omega_{X} \otimes_{\varpi^{-1} \mathscr{O}_{X}} \mathscr{O}_{\widetilde{X}}^{\mathrm{t}}$, an object of $\mathrm{D}^{\mathrm{b}}\left(\mathrm{I}\left(\left(\mathscr{D}_{\widetilde{X}}^{\mathcal{A}}\right)^{\mathrm{op}}\right)\right)$.

For $\mathscr{M} \in \mathrm{D}^{\mathrm{b}}\left(\mathscr{D}_{X}\right)$ we set:

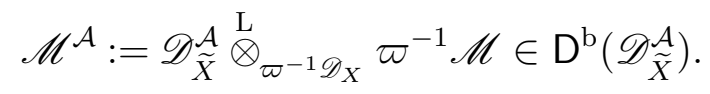

Lemma 4.2.3. For $\mathscr{M} \in \mathrm{D}^{\mathrm{b}}\left(\mathscr{D}_{X}\right)$, we have

$$
\begin{aligned}
& \varpi^{!} \mathcal{D} \mathcal{R}_{X}^{\mathrm{t}}(\mathscr{M}(* D)) \simeq \mathcal{D} \mathcal{R}_{\widetilde{X}}^{\mathrm{t}}\left(\mathscr{M}^{\mathcal{A}}\right) \\
& \mathrm{R} \varpi_{*} \mathcal{D} \mathcal{R}_{\widetilde{X}}^{\mathrm{t}}\left(\mathscr{M}^{\mathcal{A}}\right) \simeq \mathcal{D} \mathcal{R}_{X}^{\mathrm{t}}(\mathscr{M}(* D))
\end{aligned}
$$


Proof. By (4.2.6), we have

$$
\begin{aligned}
& \varpi^{!} \mathcal{D} \mathcal{R}_{X}^{\mathrm{t}}(\mathscr{M}(* D)) \simeq \varpi^{!}\left(\Omega_{X}^{\mathrm{t}} \stackrel{\mathrm{L}}{\otimes}_{\mathscr{D}_{X}} \mathscr{M}(* D)\right) \\
& \simeq \varpi^{!}\left(\Omega_{X}^{\mathrm{t}}(* D) \stackrel{\mathrm{L}}{\otimes_{\mathscr{D}_{X}}} \mathscr{M}\right) \\
& \simeq\left(\varpi^{!} \Omega_{X}^{\mathrm{t}}(* D)\right) \stackrel{\mathrm{L}}{\otimes}_{\varpi^{-1} \mathscr{D}_{X}} \varpi^{-1} \mathscr{M} \\
& \simeq \Omega_{\widetilde{X}}^{\mathrm{t}} \stackrel{\mathrm{L}}{\otimes_{\mathscr{D} \mathcal{X}}} \mathscr{D}_{\tilde{X}}^{\mathcal{A}} \stackrel{\mathrm{L}}{\varpi}_{\varpi^{-1} \mathscr{D}_{X}} \varpi^{-1} \mathscr{M} \\
& \simeq \Omega_{\widetilde{X}}^{\mathrm{t}} \stackrel{\mathrm{L}}{\otimes_{\mathscr{D} \mathcal{A}}} \mathscr{M}^{\mathcal{A}} \simeq \mathcal{D} \mathcal{R}_{\widetilde{X}}^{\mathrm{t}}\left(\mathscr{M}^{\mathcal{A}}\right) .
\end{aligned}
$$

Hence we obtain the first isomorphism.

Since

$$
\mathcal{D R}_{X}^{\mathrm{t}}(\mathscr{M}(* D)) \stackrel{\sim}{\longrightarrow} \mathscr{I h o m}\left(\mathbb{C}_{X \backslash D}, \mathcal{D} \mathcal{R}_{X}^{\mathrm{t}}(\mathscr{M}(* D))\right),
$$

the second isomorphism follows from Lemma 4.2.2.

Q.E.D.

Proposition 4.2.4. Let $\mathscr{L}$ be a holonomic $\mathscr{D}_{X}$-module with regular normal form along $D$. Then, locally on $\widetilde{X}$,

$$
\mathscr{L}^{\mathcal{A}} \simeq \mathcal{A}_{\tilde{X}} \simeq \mathscr{O}_{X}^{\mathcal{A}} \text { in } \mathrm{D}^{\mathrm{b}}\left(\mathscr{D}_{\tilde{X}}^{\mathcal{A}}\right)
$$

Proof. Let us keep the notations of Definition 4.1.2. We may assume that $\mathscr{L}=\mathscr{D}_{X} / \mathscr{I}_{\lambda}$. Since $z^{\lambda}:=\prod_{i=1}^{r} z_{i}^{\lambda_{i}}$ is a locally invertible section of $\mathcal{A}_{\tilde{X}}$, the result follows from

$$
\left(z_{i} \partial_{i}-\lambda_{i}\right) z^{\lambda}=z^{\lambda} z_{i} \partial_{i}
$$

Q.E.D.

\subsection{Regular Riemann-Hilbert correspondence}

We shall first prove the regularity theorem for regular holonomic D-modules, namely, any solution of such a D-module is tempered.

Theorem 4.3.1. Let $\mathscr{M} \in \mathrm{D}_{\mathrm{rh}}^{\mathrm{b}}\left(\mathscr{D}_{X}\right)$. Then there are isomorphisms:

$$
\begin{aligned}
& \mathcal{D R}_{X}^{\mathrm{t}}(\mathscr{M}) \stackrel{\sim}{\sim} \mathcal{D R}_{X}(\mathscr{M}) \text { in } \mathrm{D}^{\mathrm{b}}\left(\mathrm{IC}_{X}\right), \\
& \mathcal{S}_{X}^{\mathrm{t}}(\mathscr{M}) \stackrel{\sim}{\sim} \mathcal{S}_{X}(\mathscr{M}) \text { in } \mathrm{D}^{\mathrm{b}}\left(\mathrm{IC}_{X}\right) .
\end{aligned}
$$


Proof. (i) Note that, thanks to (3.1.1), the isomorphism in (4.3.2) is equivalent to the isomorphism in (4.3.1) for $\mathbb{D}_{X} \mathscr{M}$. We shall only prove (4.3.1).

(ii) We shall apply Lemma 4.1.4. Denote by $P_{X}(\mathscr{M})$ the statement which asserts that the morphism in (4.3.1) is an isomorphism.

(a)-(d) of this lemma are clearly satisfied.

(e) follows from isomorphism (3.1.9) in Corollary 3.1.6 and its non-tempered version, isomorphism (1.2.5) in Theorem 1.2.1.

(f) Let us check property (f). Let $\mathscr{M}$ be a holonomic $\mathscr{D}_{X}$-module with regular normal form along a normal crossing divisor $D$.

We want to prove the isomorphism $\mathcal{D} \mathcal{R}_{X}^{\mathrm{t}}(\mathscr{M}) \stackrel{\sim}{\longrightarrow} \alpha_{X} \mathcal{D} \mathcal{R}_{X}^{\mathrm{t}}(\mathscr{M})$. Since $\mathrm{R} \varpi_{*} \mathcal{D} \mathcal{R}_{\widetilde{X}}^{\mathrm{t}}\left(\mathscr{M}^{\mathcal{A}}\right) \stackrel{\sim}{\sim} \mathcal{D} \mathcal{R}_{X}^{\mathrm{t}}(\mathscr{M})$ by Lemma 4.2 .3 and since $\mathrm{R} \varpi_{*}$ commutes with $\alpha$, we are reduced to prove the isomorphism

$$
\mathcal{D} \mathcal{R}_{\widetilde{X}}^{\mathrm{t}}\left(\mathscr{M}^{\mathcal{A}}\right) \stackrel{\sim}{\longrightarrow} \alpha_{\tilde{X}} \mathcal{D} \mathcal{R}_{\widetilde{X}}^{\mathrm{t}}\left(\mathscr{M}^{\mathcal{A}}\right) .
$$

This is a local problem on $\widetilde{X}$ and we may apply Proposition 4.2.4. Hence it is enough to show

$$
\mathcal{D} \mathcal{R}_{\widetilde{X}}^{\mathrm{t}}\left(\mathscr{O}_{X}^{\mathcal{A}}\right) \stackrel{\sim}{\longrightarrow} \alpha_{\tilde{X}} \mathcal{D} \mathcal{R}_{\widetilde{X}}^{\mathrm{t}}\left(\mathscr{O}_{X}^{\mathcal{A}}\right)
$$

which follows from

$$
\mathcal{D} \mathcal{R}_{\widetilde{X}}^{\mathrm{t}}\left(\mathscr{O}_{X}^{\mathcal{A}}\right) \simeq \mathbb{C}_{\widetilde{X}}\left[d_{X}\right] .
$$

This completes the proof of property (f).

Q.E.D.

The following theorem is a generalized form of the Riemann-Hilbert correspondence for regular holonomic D-modules (see Remark 4.3.3).

Theorem 4.3.2 (Generalized regular Riemann-Hilbert correspondence). Let $\mathscr{M} \in \mathrm{D}_{\mathrm{rh}}^{\mathrm{b}}\left(\mathscr{D}_{X}\right)$. There is an isomorphism functorial in $\mathscr{M}$

$$
\mathscr{O}_{X}^{\mathrm{t}} \stackrel{\mathrm{D}}{\otimes} \mathscr{M} \stackrel{\mathrm{R}}{\longrightarrow} \mathscr{I h o m}\left(\mathcal{S}_{\text {ol }}^{\mathrm{t}}(\mathscr{M}), \mathscr{O}_{X}^{\mathrm{t}}\right) \text { in } \mathrm{D}^{\mathrm{b}}\left(\mathrm{I} \mathscr{D}_{X}\right) .
$$

Proof. (i) The morphism in (4.3.3) is obtained by adjunction from the composition of the morphisms

$$
\mathscr{O}_{X}^{\mathrm{t}} \stackrel{\mathrm{D}}{\otimes} \mathscr{M} \stackrel{\mathrm{L}}{\otimes} \mathrm{R} \mathscr{I} \operatorname{hom}_{\mathscr{D}_{X}}\left(\mathscr{M}, \mathscr{O}_{X}^{\mathrm{t}}\right) \rightarrow \mathscr{O}_{X}^{\mathrm{t}} \stackrel{\mathrm{L}}{\otimes_{\beta} \mathscr{O}_{X}} \mathscr{O}_{X}^{\mathrm{t}} \rightarrow \mathscr{O}_{X}^{\mathrm{t}} .
$$

(ii) We shall apply Lemma 4.1.4. Denote by $P_{X}(\mathscr{M})$ the statement which asserts that the morphism in (4.3.3) is an isomorphism. 
Properties (a)-(d) of this lemma are clearly satisfied.

(e) By Corollary 3.1.7, we have

$$
\mathscr{O}_{Y}^{\mathrm{t}} \stackrel{\mathrm{D}}{\otimes} \mathrm{D} f_{*} \mathscr{M} \simeq \mathrm{D} f_{*}\left(\mathscr{O}_{X}^{\mathrm{t}} \stackrel{\mathrm{D}}{\otimes} \mathscr{M}\right) .
$$

On the other hand we have

$$
\mathcal{S}_{o l}^{\mathrm{t}}\left(\mathrm{D} f_{*} \mathscr{M}\right) \simeq \mathrm{R} f_{! !} \mathcal{S}_{o l}^{\mathrm{t}}(\mathscr{M})\left[d_{X}-d_{Y}\right]
$$

by (3.1.1), (3.1.9) and Theorem 1.2.2 (i). Hence we have

$$
\begin{aligned}
\operatorname{R} \mathscr{I h o m} & \left(\mathcal{S}_{\sin }^{\mathrm{t}}\left(\mathrm{D} f_{*} \mathscr{M}\right), \mathscr{O}_{Y}^{\mathrm{t}}\right) \\
& \simeq \operatorname{R} \mathscr{I h o m}\left(\mathrm{R} f_{! !} \mathcal{S o l}_{X}^{\mathrm{t}}(\mathscr{M})\left[d_{X}-d_{Y}\right], \mathscr{O}_{Y}^{\mathrm{t}}\right) \\
& \simeq \operatorname{R} f_{*} \operatorname{R} \mathscr{I h o m}\left(\mathcal{S}_{l_{X}}^{\mathrm{t}}(\mathscr{M})\left[d_{X}-d_{Y}\right], f^{!} \mathscr{O}_{Y}^{\mathrm{t}}\right) .
\end{aligned}
$$

By (3.1.3), we have

$$
f^{!} \mathscr{O}_{Y}^{\mathrm{t}} \simeq \mathscr{D}_{Y \leftarrow X} \stackrel{\mathrm{L}}{\mathscr{Q}_{\mathscr{D}_{X}}} \mathscr{O}_{X}^{\mathrm{t}}\left[d_{X}-d_{Y}\right] .
$$

Hence we have

$$
\begin{aligned}
& \mathrm{R} \mathscr{I h o m}\left(\mathcal{S}_{\operatorname{lol}}^{\mathrm{t}}\left(\mathrm{D} f_{*} \mathscr{M}\right), \mathscr{O}_{Y}^{\mathrm{t}}\right) \\
& \simeq \operatorname{R} f_{*} \mathrm{R} \mathscr{I h o m}\left(\operatorname{Sol}_{X}^{\mathrm{t}}(\mathscr{M}), \mathscr{D}_{Y \leftarrow X} \stackrel{\mathrm{L}}{\mathscr{D}_{\mathscr{D}_{X}}} \mathscr{O}_{X}^{\mathrm{t}}\right) \\
& \simeq \operatorname{R} f_{*}\left(\mathscr{D}_{Y \leftarrow X} \stackrel{\mathrm{L}}{\otimes}_{\mathscr{D}_{X}} \operatorname{R} \mathscr{I h o m}\left(\mathcal{S}_{l_{X}}^{\mathrm{t}}(\mathscr{M}), \mathscr{O}_{X}^{\mathrm{t}}\right)\right) \\
& \simeq \mathrm{D} f_{*} \operatorname{R} \mathscr{I h o m}\left(\mathcal{S}_{\text {ol }}^{\mathrm{t}}(\mathscr{M}), \mathscr{O}_{X}^{\mathrm{t}}\right) \text {. }
\end{aligned}
$$

Combining with (4.3.5), we finally obtain

$$
\begin{aligned}
\mathscr{O}_{Y}^{\mathrm{t}} \stackrel{\mathrm{D}}{\otimes} \mathrm{D} f_{*} \mathscr{M} & \simeq \mathrm{D} f_{*}\left(\mathscr{O}_{X}^{\mathrm{t}} \stackrel{\mathrm{D}}{\otimes} \mathscr{M}\right) \\
& \simeq \mathrm{D} f_{*} \operatorname{R} \mathscr{I h o m}\left(\mathcal{S}_{o l}^{\mathrm{t}}(\mathscr{M}), \mathscr{O}_{X}^{\mathrm{t}}\right) \\
& \simeq \operatorname{R} \mathscr{I} h o m\left(\mathcal{S o l}_{Y}^{\mathrm{t}}\left(\mathrm{D} f_{*} \mathscr{M}\right), \mathscr{O}_{Y}^{\mathrm{t}}\right) .
\end{aligned}
$$

Here the second isomorphism follows from $P_{X}(\mathscr{M})$.

(f) Let us check property (f) for (4.3.3). Hence, we assume that $\mathscr{M}$ has regular normal form along $D$. 
By Lemmas 4.1.3 and 3.2.1 we have

$$
\begin{aligned}
& \operatorname{R} \mathscr{I h o m}\left(\mathcal{S}_{\operatorname{lol}}(\mathscr{M}), \mathscr{O}_{X}^{\mathrm{t}}\right) \simeq \operatorname{R} \mathscr{I h o m}\left(\mathcal{S o l}_{X}(\mathscr{M}) \otimes \mathbb{C}_{X \backslash D}, \mathscr{O}_{X}^{\mathrm{t}}\right) \\
& \simeq \operatorname{R} \mathscr{I h o m}\left(\mathcal{S}_{S}(\mathscr{M}), \operatorname{R} \mathscr{I h o m}\left(\mathbb{C}_{X \backslash D}, \mathscr{O}_{X}^{\mathrm{t}}\right)\right) \\
& \simeq \operatorname{R} \mathscr{I h o m}\left(\mathcal{S}_{l_{X}}(\mathscr{M}), \operatorname{R} \varpi_{*} \mathscr{O}_{\widetilde{X}}^{\mathrm{t}}\right) \\
& \simeq \mathrm{R} \varpi_{*} \mathrm{R} \mathscr{I h o m}\left(\varpi^{-1} \mathcal{S}_{X}(\mathscr{M}), \mathscr{O}_{\widetilde{X}}^{\mathrm{t}}\right) \\
& \simeq \operatorname{R} \varpi_{*} \operatorname{R} \mathscr{I h o m}\left(\mathcal{S}_{\tilde{X}}\left(\mathscr{M}^{\mathcal{A}}\right), \mathscr{O}_{\widetilde{X}}^{\mathrm{t}}\right) \text {. }
\end{aligned}
$$

Here the last isomorphism follows from

$$
\mathbb{C}_{\tilde{X}>0} \stackrel{\mathrm{L}}{\otimes} \varpi^{-1} \mathcal{S}_{l_{X}}(\mathscr{M}) \simeq \mathbb{C}_{\tilde{X}>0} \stackrel{\mathrm{L}}{\otimes} \mathcal{S}_{\widetilde{X}^{\prime}}\left(\mathscr{M}^{\mathcal{A}}\right) .
$$

On the other-hand, we have

$$
\begin{aligned}
\mathscr{O}_{X}^{\mathrm{t}} \stackrel{\mathrm{D}}{\otimes} \mathscr{M} \simeq \mathscr{O}_{X}^{\mathrm{t}}(* D) \stackrel{\mathrm{D}}{\otimes} \mathscr{M} & \simeq\left(\mathrm{R} \varpi_{*} \mathscr{O}_{\widetilde{X}}^{\mathrm{t}}\right)^{\mathrm{D}} \otimes \mathscr{M} \\
& \simeq \operatorname{R} \varpi_{*}\left(\mathscr{O}_{\widetilde{X}}^{\mathrm{t}} \stackrel{\mathrm{Q}}{\varpi}_{\varpi^{-1} \mathscr{O}_{X}} \varpi^{-1} \mathscr{M}\right) \\
& \simeq \mathrm{R} \varpi_{*}\left(\mathscr{O}_{\widetilde{X}}^{\mathrm{t}}{\stackrel{\mathrm{L}}{\mathcal{A}_{\tilde{X}}}} \mathscr{M}^{\mathcal{A}}\right) .
\end{aligned}
$$

Hence it is enough to show that

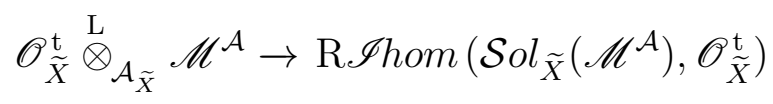

is an isomorphism. Note that this morphism is obtained from a similar morphism to (4.3.4) by adjunction. By Proposition 4.2.4, $\mathscr{M}^{\mathcal{A}}$ is locally isomorphic to $\mathcal{A}_{\tilde{X}}$. Then $\operatorname{Sol}_{\tilde{X}}\left(\mathscr{M}^{\mathcal{A}}\right) \simeq \mathbb{C}_{\tilde{X}}$, and it is obvious that (4.3.6) is an isomorphism.

Q.E.D.

Remark 4.3.3. Isomorphism (4.3.1) already appeared in [Ka 84]. Isomorphism (4.3.3) (with a different formulation) is essentially due to Björk [Bj 93, Th. 7.9.11].

Applying the functor $\alpha_{X}$ to isomorphism (4.3.3), we get the RiemannHilbert correspondence for regular holonomic D-modules: 
Corollary 4.3.4 (Regular Riemann-Hilbert correspondence [Ka 80]). Let $\mathscr{M} \in \mathrm{D}_{\mathrm{rh}}^{\mathrm{b}}\left(\mathscr{D}_{X}\right)$. There is an isomorphism in $\mathrm{D}^{\mathrm{b}}\left(\mathscr{D}_{X}\right)$ :

$$
\mathscr{M} \simeq \mathrm{R} \mathscr{H} o m_{\mathbb{I C}_{X}}\left(\mathcal{S}_{o l}(\mathscr{M}), \mathscr{O}_{X}^{\mathrm{t}}\right) .
$$

Corollary 4.3.5. Let $\mathscr{M} \in \mathrm{D}_{\mathrm{rh}}^{\mathrm{b}}\left(\mathscr{D}_{X}\right)$ and let $\mathscr{L} \in \mathrm{D}^{\mathrm{b}}\left(\mathscr{D}_{X}\right)$. Then isomorphism (4.3.3) induces the isomorphism

$$
\mathcal{D R}^{\mathrm{t}}(\mathscr{L} \stackrel{\mathrm{D}}{\otimes} \mathscr{M}) \simeq \mathrm{R} \mathscr{I h o m}\left(\operatorname{Sol}_{X}^{\mathrm{t}}(\mathscr{M}), \mathcal{D} \mathcal{R}^{\mathrm{t}}(\mathscr{L})\right) .
$$

Proof. We have

$$
\begin{aligned}
\mathcal{D R}^{\mathrm{t}}(\mathscr{L} \stackrel{\mathrm{D}}{\otimes} \mathscr{M}) & =\Omega_{X}^{\mathrm{t}} \stackrel{\mathrm{L}}{\otimes_{\mathscr{D}_{X}}}(\mathscr{L} \stackrel{\mathrm{D}}{\otimes} \mathscr{M}) \\
& \simeq\left(\Omega_{X}^{\mathrm{t}} \stackrel{\mathrm{D}}{\otimes} \mathscr{M}\right) \stackrel{\mathrm{L}}{\otimes}_{\mathscr{D}_{X}} \mathscr{L} \\
& \simeq \mathrm{R} \mathscr{I} h o m\left(\mathcal{S}_{X}^{\mathrm{t}}(\mathscr{M}), \Omega_{X}^{\mathrm{t}}\right) \stackrel{\mathrm{L}}{\otimes}_{\mathscr{D}_{X}} \mathscr{L} \\
& \simeq \operatorname{R} \mathscr{I h o m}\left(\mathcal{S}_{X}^{\mathrm{t}}(\mathscr{M}), \Omega_{X}^{\mathrm{t}}{\stackrel{\mathrm{L}}{\mathscr{D}_{X}}}_{\mathscr{L}} \mathscr{L}\right) .
\end{aligned}
$$

Here, the last isomorphism follows from Theorem 2.3.6, using the fact that $\operatorname{Sol}^{\mathrm{t}}(\mathscr{M}) \stackrel{\sim}{\longrightarrow} \operatorname{Sol}(\mathscr{M})$.

Q.E.D.

As an application of isomorphism (4.3.2), we get:

Corollary 4.3.6. Let $\mathscr{M} \in \mathrm{D}_{\mathrm{rh}}^{\mathrm{b}}\left(\mathscr{D}_{X}\right)$ and let $F \in \mathrm{D}_{\mathbb{R}_{-c}}^{\mathrm{b}}\left(\mathbb{C}_{X}\right)$. Then we have the natural isomorphism

$$
\mathrm{R} \mathscr{H} o m_{\mathscr{D}_{X}}\left(\mathscr{M}, \mathrm{R} \mathscr{H} o m_{\mathbb{I C}_{X}}\left(F, \mathscr{O}_{X}^{\mathrm{t}}\right)\right) \stackrel{\sim}{\longrightarrow} \operatorname{R} \mathscr{H} o m_{\mathscr{D}_{X}}\left(\mathscr{M}, \operatorname{R} \mathscr{H} o m\left(F, \mathscr{O}_{X}\right)\right) .
$$

Let $M$ be a real analytic manifold and $X$ a complexification of $M$. Choosing for $F$ the object $\mathrm{D}_{X}^{\prime} \mathbb{C}_{M}$, we get the isomorphism between the complexes of distribution solutions and hyperfunction solutions of $\mathscr{M}$ :

$$
\mathrm{R} \mathscr{H} o m_{\mathscr{D}_{X}}\left(\mathscr{M}, \mathcal{D} b_{M}\right) \stackrel{\sim}{\longrightarrow} \mathscr{H}_{o} m_{\mathscr{D}_{X}}\left(\mathscr{M}, \mathscr{B}_{M}\right) .
$$

Remark 4.3.7. Of course, isomorphism (4.3.3) is no more true if one replaces $\mathscr{O}_{X}^{\mathrm{t}}$ with $\mathscr{O}_{X}$. For example, choosing $\mathscr{M}=\mathscr{O}_{X}(* Y)$ for $Y$ a closed hypersurface, the left-hand is the sheaf of meromorphic functions with poles on $Y$ and the right-hand side the sheaf of holomorphic functions with possibly essential singularities on $Y$. 


\subsection{Integral transforms with regular kernels}

Consider morphisms of complex manifolds

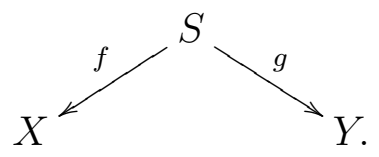

Notation 4.4.1. (i) For $\mathscr{M} \in \mathrm{D}^{\mathrm{b}}\left(\mathscr{D}_{X}\right)$ and $\mathscr{L} \in \mathrm{D}^{\mathrm{b}}\left(\mathscr{D}_{S}\right)$ one sets

$$
\mathscr{M} \stackrel{\mathrm{D}}{\circ} \mathscr{L}:=\mathrm{D} g_{*}\left(\mathrm{D} f^{*} \mathscr{M} \stackrel{\mathrm{D}}{\otimes} \mathscr{L}\right)
$$

(ii) For $L \in \mathrm{D}^{\mathrm{b}}\left(\mathrm{I}_{S}\right), F \in \mathrm{D}^{\mathrm{b}}\left(\mathrm{I} \mathbb{C}_{X}\right)$ and $G \in \mathrm{D}^{\mathrm{b}}\left(\mathrm{I}_{Y}\right)$ one sets

$$
\begin{aligned}
& L \circ G:=\mathrm{R} f_{! !}\left(L \otimes g^{-1} G\right), \\
& \Phi_{L}(G)=L \circ G, \quad \Psi_{L}(F)=\mathrm{R} g_{*} \operatorname{R} \mathscr{I h o m}\left(L, f^{!} F\right) .
\end{aligned}
$$

Note that we have a pair of adjoint functors

$$
\Phi_{L}: \mathrm{D}^{\mathrm{b}}\left(\mathrm{I}_{Y}\right) \rightleftarrows \mathrm{D}^{\mathrm{b}}\left(\mathrm{IC}_{X}\right): \Psi_{L}
$$

Theorem 4.4.2. Let $\mathscr{M} \in \mathrm{D}_{\mathrm{q}-\operatorname{good}}^{\mathrm{b}}\left(\mathscr{D}_{X}\right)$, let $\mathscr{L} \in \mathrm{D}_{\mathrm{rh}}^{\mathrm{b}}\left(\mathscr{D}_{S}\right)$ and set $L:=$ $\operatorname{Sol}_{S}(\mathscr{L})$. Assume that $f^{-1} \operatorname{Supp}(\mathscr{M}) \cap \operatorname{Supp}(\mathscr{L})$ is proper over $Y$ and that $\mathscr{L}$ is good. Then there is a natural isomorphism in $\mathrm{D}^{\mathrm{b}}\left(\mathrm{IC}_{Y}\right)$ :

$$
\Psi_{L}\left(\mathcal{D} \mathcal{R}_{X}^{\mathrm{t}}(\mathscr{M})\right)\left[d_{X}-d_{S}\right] \simeq \mathcal{D} \mathcal{R}_{Y}^{\mathrm{t}}(\mathscr{M} \stackrel{\mathrm{D}}{\circ} \mathscr{L}) .
$$

Note that any regular holonomic $\mathscr{D}$-module is good.

Proof. Applying Corollaries 3.1.2, 3.1.6 and 4.3.5, we get:

$$
\begin{aligned}
\mathcal{D} \mathcal{R}_{Y}^{\mathrm{t}}(\mathscr{M} \stackrel{\mathrm{D}}{\circ} \mathscr{L}) & =\mathcal{D} \mathcal{R}_{Y}^{\mathrm{t}}\left(\mathrm{D} g_{*}\left(\mathrm{D} f^{*} \mathscr{M} \stackrel{\mathrm{D}}{\otimes} \mathscr{L}\right)\right) \\
& \simeq \operatorname{R} g_{*} \mathcal{D} \mathcal{R}_{S}^{\mathrm{t}}\left(\mathrm{D} f^{*} \mathscr{M} \stackrel{\mathrm{D}}{\otimes} \mathscr{L}\right) \\
& \simeq \operatorname{R} g_{*} \operatorname{R} \mathscr{I} h o m\left(\mathcal{S} o l_{S}^{\mathrm{t}}(\mathscr{L}), \mathcal{D} \mathcal{R}_{S}^{\mathrm{t}}\left(\mathrm{D} f^{*} \mathscr{M}\right)\right) \\
& \simeq \operatorname{R} g_{*} \operatorname{R} \mathscr{I} h o m\left(L, f^{!} \mathcal{D} \mathcal{R}_{X}^{\mathrm{t}}(\mathscr{M})\right)\left[d_{X}-d_{S}\right] \\
& =\Psi_{L}\left(\mathcal{D} \mathcal{R}_{X}^{\mathrm{t}}(\mathscr{M})\right)\left[d_{X}-d_{S}\right] .
\end{aligned}
$$

Q.E.D. 
By applying the functor $\operatorname{RHom}(G, \bullet)$ with $G \in \mathrm{D}^{\mathrm{b}}\left(\mathrm{IC}_{Y}\right)$ to both sides of (4.4.3), one gets

Corollary 4.4.3 ([KS01, Th. 7.4.13]). Let $\mathscr{M} \in \mathrm{D}_{\text {q-good }}^{\mathrm{b}}\left(\mathscr{D}_{X}\right)$, let $\mathscr{L} \in$ $\mathrm{D}_{\mathrm{rh}}^{\mathrm{b}}\left(\mathscr{D}_{S}\right)$ and let $L:=\operatorname{Sol}_{S}(\mathscr{L})$. Assume that $f^{-1} \operatorname{Supp}(\mathscr{M}) \cap \operatorname{Supp}(\mathscr{L})$ is proper over $Y$ and that $\mathscr{L}$ is good. Let $G \in \mathrm{D}^{\mathrm{b}}\left(\mathrm{I}_{Y}\right)$. Then one has the isomorphism

$$
\begin{aligned}
\operatorname{RHom}_{I_{X}}(L \circ G, & \left.\mathcal{D} \mathcal{R}_{X}^{\mathrm{t}}(\mathscr{M})\right)\left[d_{X}-d_{S}\right] \\
& \simeq \operatorname{RHom}_{\mathbb{I C}_{Y}}\left(G, \mathcal{D} \mathcal{R}_{Y}^{\mathrm{t}}(\mathscr{M} \circ \mathscr{L})\right) .
\end{aligned}
$$

Note that a similar formula holds when replacing $\mathscr{O}_{X}^{\mathrm{t}}$ and $\mathscr{O}_{Y}^{\mathrm{t}}$ with their non tempered versions $\mathscr{O}_{X}$ and $\mathscr{O}_{Y}$ (and indsheaves with usual sheaves), but the hypotheses are different. Essentially, $\mathscr{M}$ has to be coherent, $f$ non characteristic for $\mathscr{M}$ and $\mathrm{D} f^{*} \mathscr{M}$ has to be transversal to the holonomic module $\mathscr{L}$. On the other hand, we do not need the regularity assumption on $\mathscr{L}$. See [DS96] for such a non tempered formula (in a more particular setting).

However, if one removes the hypothesis that the holonomic module $\mathscr{L}$ is regular in Theorem 4.4.2, formula (4.4.3) does not hold anymore and we have to replace $\mathscr{O}_{X}^{\mathrm{t}}$ with its enhanced version, as we shall see in the next sections.

\subsection{Irregular D-modules: an example}

In this subsection we recall an example treated in [KS 03] which emphasizes the role of the sheaf $\mathscr{O}_{X}^{\mathrm{t}}$ in the study of irregular holonomic D-modules.

Let $X=\mathbb{C}$ endowed with the holomorphic coordinate $z$. Define

$$
U=X \backslash\{0\}, \quad j: U \hookrightarrow X \text { the open embedding. }
$$

Consider the differential operator $P=z^{2} \partial_{z}+1$ and the $\mathscr{D}_{X}$-module $\mathscr{L}:=$ $\mathscr{D}_{X} \exp (1 / z) \simeq \mathscr{D}_{X} / \mathscr{D}_{X} P$.

Notice first that $\mathscr{O}_{X}^{\mathrm{t}}$ is concentrated in degree 0 (since $\operatorname{dim} X=1$ ) and it is a sub-indsheaf of $\mathscr{O}_{X}$. Therefore the morphism $H^{0}\left(\mathcal{S}_{\text {ol }}^{\mathrm{t}}(\mathscr{L})\right) \rightarrow$ $H^{0}\left(\mathcal{S o l}_{X}(\mathscr{L})\right) \simeq \mathbb{C}_{U}$ is a monomorphism. It follows that for $V \subset X \backslash\{0\}$ a connected open subset, $\Gamma\left(V ; H^{0} \mathcal{S} o l^{\mathrm{t}}(\mathscr{M})\right) \neq 0$ if and only if $V \subset U$ and $\left.\exp (1 / z)\right|_{V}$ is tempered.

Denote by $\bar{B}_{\varepsilon}$ the closed ball with center $(\varepsilon, 0)$ and radius $\varepsilon$ and set

$$
U_{\varepsilon}=X \backslash \bar{B}_{\varepsilon}=\{z \in \mathbb{C} \backslash\{0\} ; \operatorname{Re}(1 / z)<1 / 2 \varepsilon\} .
$$


One proves that $\exp (1 / z)$ is tempered (in a neighborhood of 0 ) on an open subanalytic subset $V \subset X \backslash\{0\}$ if and only if $\operatorname{Re}(1 / z)$ is bounded on $V$, that is, if and only if $V \subset U_{\varepsilon}$ for some $\varepsilon>0$. We get the isomorphism

$$
\operatorname{Sol}^{\mathrm{t}}(\mathscr{L}) \otimes \mathbb{C}_{U} \simeq \underset{\varepsilon>0}{\stackrel{\lim }{\longrightarrow}} \mathbb{C}_{U_{\varepsilon}}
$$

Note that $\mathcal{S o l}^{\mathrm{t}}(\mathscr{L}) \otimes \mathbb{C}_{U}$ is concentrated in degree 0 .

Since $\mathcal{S}_{\text {ol }}{ }^{\mathrm{t}}(\mathscr{L}) \simeq \mathcal{D} \mathcal{R}^{\mathrm{t}}(\mathbb{D} \mathscr{L})$ and $\mathbb{D} \mathscr{L} \simeq \mathbb{D} \mathscr{L}(*\{0\})$, we get that

$$
\operatorname{Sol}^{\mathrm{t}}(\mathscr{L}) \simeq \mathrm{R} \mathscr{I h o m}\left(\mathbb{C}_{U}, \operatorname{Sol}^{\mathrm{t}}(\mathscr{L})\right) \simeq \mathrm{R} \mathscr{I h o m}\left(\mathbb{C}_{U}, \operatorname{Sol}^{\mathrm{t}}(\mathscr{L}) \otimes \mathbb{C}_{U}\right) .
$$

Therefore,

$$
\begin{aligned}
& \operatorname{Sol}^{\mathrm{t}}(\mathscr{L}) \simeq \operatorname{R} \mathscr{I h o m}\left(\mathbb{C}_{U}, \underset{\varepsilon>0}{ } \underset{\mathrm{lim}}{\longrightarrow} \mathbb{C}_{U_{\varepsilon}}\right), \\
& H^{0}\left(\mathcal{S o l}^{\mathrm{t}}(\mathscr{L})\right) \simeq \underset{\varepsilon>0}{\stackrel{\lim }{\longrightarrow}} \mathbb{C}_{U_{\varepsilon}}, \\
& H^{1}\left(\mathcal{S o l}^{\mathrm{t}}(\mathscr{L})\right) \simeq \underset{\varepsilon>0}{\stackrel{\lim }{\longrightarrow}} \mathscr{E} x t^{1}\left(\mathbb{C}_{U}, \mathbb{C}_{U_{\varepsilon}}\right) \simeq \mathbb{C}_{\{0\}}, \\
& \operatorname{Sol}(\mathscr{L}) \simeq \alpha_{X} \operatorname{Sol}^{\mathrm{t}}(\mathscr{L}) \simeq \operatorname{R} \mathscr{H} o m\left(\mathbb{C}_{U}, \mathbb{C}_{U}\right), \\
& H^{0}(\operatorname{Sol}(\mathscr{L})) \simeq \mathbb{C}_{U}, \quad H^{1}(\operatorname{Sol}(\mathscr{L})) \simeq \mathbb{C}_{\{0\}} .
\end{aligned}
$$

The functor $\mathcal{S}_{\text {ol }}{ }^{\mathrm{t}}$ is not fully faithful since the $\mathscr{D}_{X}$-modules $\mathscr{D}_{X} \exp (1 / z)$ and $\mathscr{D}_{X} \exp (2 / z)$ have the same indsheaves of tempered holomorphic solutions although they are not isomorphic.

However, $\mathcal{S}_{\text {ol }}^{\mathrm{t}}\left(\mathscr{D}_{X} \exp (1 / z)\right) \not \mathcal{\sim} \mathcal{S}_{\text {ol }}^{\mathrm{t}}\left(\mathscr{D}_{X} \exp \left(1 / z^{m}\right)\right)$ for any $m>1$.

Hence, the functor $\mathcal{S}_{\text {ol }}{ }^{\mathrm{t}}$ is sensitive enough to distinguish $m \in \mathbb{Z}_{>0}$ in $\mathscr{D}_{X} \exp \left(z^{-m}\right)$ but it is not sensitive enough to distinguish $c \in \mathbb{R}_{>0}$ in $\mathscr{D}_{X} \exp \left(c z^{-1}\right)$.

In order to capture $c$, we need to work in the framework of enhanced indsheaves, which we are going to explain in the next sections.

\section{Indsheaves on bordered spaces}

\subsection{Bordered spaces}

Definition 5.1.1. The category of bordered spaces is the category whose objects are pairs $(M, \widehat{M})$ with $M \subset \widehat{M}$ an open embedding of good topological 
spaces. Morphisms $f:(M, \widehat{M}) \rightarrow(N, \widehat{N})$ are continuous maps $f: M \rightarrow N$ such that

$$
\bar{\Gamma}_{f} \rightarrow \widehat{M} \text { is proper. }
$$

Here $\Gamma_{f} \subset M \times N$ is the graph of $f$ an $\bar{\Gamma}_{f}$ is its closure in $\widehat{M} \times \widehat{N}$.

The composition of $(L, \widehat{L}) \stackrel{g}{\rightarrow}(M, \widehat{M}) \stackrel{f}{\rightarrow}(N, \widehat{N})$ is given by $f \circ g: L \rightarrow N$ (see Lemma 5.1.2 below), and the identity $\operatorname{id}_{(M, \widehat{M})}$ is given by $\operatorname{id}_{M}$.

Lemma 5.1.2. Let $f:(M, \widehat{M}) \rightarrow(N, \widehat{N})$ and $g:(L, \widehat{L}) \rightarrow(M, \widehat{M})$ be morphisms of bordered spaces. Then the composition $f \circ g$ is a morphism of bordered spaces.

One shall identify a space $M$ and the bordered space $(M, M)$. Then, by using the identifications $M=(M, M)$ and $\widehat{M}=(\widehat{M}, \widehat{M})$, there are natural morphisms of bordered spaces

$$
M \rightarrow(M, \widehat{M}) \rightarrow \widehat{M} .
$$

Note however that $(M, \widehat{M}) \rightarrow M$ is a morphism of bordered spaces if and only if $M$ is a closed subset of $\widehat{M}$.

We can easily see that the category of bordered spaces admits products:

$$
(M, \widehat{M}) \times(N, \widehat{N}) \simeq(M \times N, \widehat{M} \times \widehat{N}) .
$$

Let $(M, \widehat{M})$ be a bordered space. Denote by $i: \widehat{M} \backslash M \rightarrow \widehat{M}$ the closed embedding. By identifying $\mathrm{D}^{\mathrm{b}}\left(\mathbf{k}_{\widehat{M} \backslash M}\right)$ with its essential image in $\mathrm{D}^{\mathrm{b}}\left(\mathbf{k}_{\widehat{M}}\right)$ by the fully faithful functor $\mathrm{R} i_{!} \simeq \mathrm{R} i_{*}$, the restriction functor $\left.F \mapsto F\right|_{M}$ induces an equivalence

$$
\mathrm{D}^{\mathrm{b}}\left(\mathbf{k}_{\widehat{M}}\right) / \mathrm{D}^{\mathrm{b}}\left(\mathbf{k}_{\widehat{M} \backslash M}\right) \stackrel{\sim}{\longrightarrow} \mathrm{D}^{\mathrm{b}}\left(\mathbf{k}_{M}\right) .
$$

This is no longer true for indsheaves. Therefore one sets

$$
\mathrm{D}^{\mathrm{b}}\left(\mathrm{I}_{(M, \widehat{M})}\right):=\mathrm{D}^{\mathrm{b}}\left(\mathrm{Ik}_{\widehat{M}}\right) / \mathrm{D}^{\mathrm{b}}\left(\mathrm{Ik}_{\widehat{M} \backslash M}\right)
$$

where $\mathrm{D}^{\mathrm{b}}\left(\mathrm{Ik}_{\widehat{M} \backslash M}\right)$ is identified with its essential image in $\mathrm{D}^{\mathrm{b}}\left(\mathrm{Ik}_{\widehat{M}}\right)$ by $\mathrm{R} i_{! !} \simeq$ $\mathrm{R} i_{*}$, as for usual sheaves.

Recall that if $\mathscr{T}$ is a triangulated category and $\mathscr{I}$ a subcategory, one denotes by ${ }^{\perp} \mathscr{I}$ and $\mathscr{I}^{\perp}$ the left and right orthogonal to $\mathscr{I}$ in $\mathscr{T}$, respectively:

$$
\begin{aligned}
{ }^{\perp} \mathscr{I} & :=\left\{A \in \mathscr{T} ; \operatorname{Hom}_{\mathscr{T}}(A, B)=0 \text { for any } B \in \mathscr{I}\right\}, \\
\mathscr{I}^{\perp} & :=\left\{A \in \mathscr{T} ; \operatorname{Hom}_{\mathscr{T}}(B, A)=0 \text { for any } B \in \mathscr{I}\right\} .
\end{aligned}
$$


Proposition 5.1.3. Let $(M, \widehat{M})$ be a bordered space. Then we have

$$
\begin{aligned}
& \mathrm{D}^{\mathrm{b}}\left(\mathrm{Ik}_{\widehat{M} \backslash M}\right)=\left\{F \in \mathrm{D}^{\mathrm{b}}\left(\mathrm{Ik}_{\widehat{M}}\right) ; \mathbf{k}_{M} \otimes F \simeq 0\right\} \\
& =\left\{F \in \mathrm{D}^{\mathrm{b}}\left(\mathrm{I}_{\widehat{M}}\right) ; \mathrm{R} \mathscr{I h o m}\left(\mathbf{k}_{M}, F\right) \simeq 0\right\}, \\
& { }^{\perp} \mathrm{D}^{\mathrm{b}}\left(\mathrm{Ik}_{\widehat{M} \backslash M}\right)=\left\{F \in \mathrm{D}^{\mathrm{b}}\left(\mathrm{Ik}_{\widehat{M}}\right) ; \mathbf{k}_{M} \otimes F \stackrel{\sim}{\longrightarrow} F\right\} \text {, } \\
& \mathrm{D}^{\mathrm{b}}\left(\mathrm{Ik}_{\widehat{M} \backslash M}\right)^{\perp}=\left\{F \in \mathrm{D}^{\mathrm{b}}\left(\mathrm{Ik}_{\widehat{M}}\right) ; F \stackrel{\mathrm{R} \mathscr{I} h o m}{\sim}\left(\mathbf{k}_{M}, F\right)\right\} .
\end{aligned}
$$

Moreover, there are equivalences

$$
\begin{aligned}
& \mathrm{D}^{\mathrm{b}}\left(\mathrm{I}_{(M, \widehat{M})}\right) \stackrel{\sim}{\longrightarrow} \mathrm{D}^{\mathrm{b}}\left(\mathrm{I}_{\widehat{M} \backslash M}\right)^{\perp}, \quad F \mapsto \operatorname{R} \mathscr{I h o m}\left(\mathbf{k}_{M}, F\right), \\
& \mathrm{D}^{\mathrm{b}}\left(\mathrm{Ik}_{(M, \widehat{M})}\right) \stackrel{\sim}{\perp} \mathrm{D}^{\mathrm{b}}\left(\mathrm{I}_{\widehat{M} \backslash M}\right), \quad F \mapsto \mathbf{k}_{M} \otimes F,
\end{aligned}
$$

with quasi-inverse induced by the quotient functor.

Corollary 5.1.4. For $F, G \in \mathrm{D}^{\mathrm{b}}\left(\mathrm{Ik}_{\widehat{M}}\right)$ one has

$$
\begin{aligned}
& \operatorname{Hom}_{\mathrm{D}^{\mathrm{b}}\left(\mathrm{Ik}_{(M, \widehat{M})}\right)}(F, G) \simeq \operatorname{Hom}_{\mathrm{D}^{\mathrm{b}}\left(\mathrm{Ik}_{\widehat{M}}\right)}\left(\mathbf{k}_{M} \otimes F, G\right) \\
& \simeq \operatorname{Hom}_{\mathrm{D}^{\mathrm{b}}\left(\mathrm{Ik}_{\widehat{M}}\right)}\left(F, \operatorname{R} \mathscr{I h o m}\left(\mathbf{k}_{M}, G\right)\right) \text {. }
\end{aligned}
$$

The functors $\otimes$ and $\mathrm{R} \mathscr{I}$ hom in $\mathrm{D}^{\mathrm{b}}\left(\mathrm{Ik}_{\widehat{M}}\right)$ induce well defined functors (we keep the same notations)

$$
\begin{aligned}
\otimes & : \quad \mathrm{D}^{\mathrm{b}}\left(\mathrm{Ik}_{(M, \widehat{M})}\right) \times \mathrm{D}^{\mathrm{b}}\left(\mathrm{Ik}_{(M, \widehat{M})}\right) \rightarrow \mathrm{D}^{\mathrm{b}}\left(\mathrm{Ik}_{(M, \widehat{M})}\right), \\
\mathrm{R} \mathscr{I} \text { hom }: & \mathrm{D}^{\mathrm{b}}\left(\mathrm{Ik}_{(M, \widehat{M})}\right)^{\mathrm{op}} \times \mathrm{D}^{\mathrm{b}}\left(\mathrm{Ik}_{(M, \widehat{M})}\right) \rightarrow \mathrm{D}^{\mathrm{b}}\left(\mathrm{Ik}_{(M, \widehat{M})}\right) .
\end{aligned}
$$

\subsection{Operations}

Let $f:(M, \widehat{M}) \rightarrow(N, \widehat{N})$ be a morphism of bordered spaces, and recall that $\Gamma_{f}$ denotes the graph of the associated map $f: M \rightarrow N$. Since $\Gamma_{f}$ is closed in $M \times N$, it is locally closed in $\widehat{M} \times \widehat{N}$. One can then consider the sheaf $\mathbf{k}_{\Gamma_{f}}$ on $\widehat{M} \times \widehat{N}$. Let $q_{1}: \widehat{M} \times \widehat{N} \rightarrow \widehat{M}$ and $q_{2}: \widehat{M} \times \widehat{N} \rightarrow \widehat{N}$ be the projections.

Definition 5.2.1. Let $f:(M, \widehat{M}) \rightarrow(N, \widehat{N})$ be a morphism of bordered spaces. For $F \in \mathrm{D}^{\mathrm{b}}\left(\mathrm{Ik}_{\widehat{M}}\right)$ and $G \in \mathrm{D}^{\mathrm{b}}\left(\mathrm{Ik}_{\widehat{N}}\right)$, we set

$$
\begin{aligned}
\mathrm{R} f_{! !} F & =\mathrm{R} q_{2 ! !}\left(\mathbf{k}_{\Gamma_{f}} \otimes q_{1}^{-1} F\right), \\
\mathrm{R} f_{*} F & =\mathrm{R} q_{2_{*}} \mathrm{R} \mathscr{I} h o m\left(\mathbf{k}_{\Gamma_{f}}, q_{1}^{!} F\right), \\
f^{-1} G & =\mathrm{R} q_{1 ! !}\left(\mathbf{k}_{\Gamma_{f}} \otimes q_{2}^{-1} G\right), \\
f^{!} G & =\mathrm{R} q_{1_{*}} \operatorname{R} \mathscr{I} h o m\left(\mathbf{k}_{\Gamma_{f}}, q_{2}^{!} G\right) .
\end{aligned}
$$


Remark 5.2.2. Considering a continuous map $f: M \rightarrow N$ as a morphism of bordered spaces with $M=\widehat{M}$ and $N=\widehat{N}$, the above functors are isomorphic to the usual external operations for indsheaves.

Lemma 5.2.3. The above definition induces well-defined functors

$$
\begin{aligned}
\mathrm{R} f_{! !}, \mathrm{R} f_{*}: \mathrm{D}^{\mathrm{b}}\left(\mathrm{Ik}_{(M, \widehat{M})}\right) & \rightarrow \mathrm{D}^{\mathrm{b}}\left(\mathrm{Ik}_{(N, \widehat{N})}\right), \\
f^{-1}, f^{!}: \mathrm{D}^{\mathrm{b}}\left(\mathrm{Ik}_{(N, \widehat{N})}\right) & \rightarrow \mathrm{D}^{\mathrm{b}}\left(\mathrm{Ik}_{(M, \widehat{M})}\right) .
\end{aligned}
$$

Lemma 5.2.4. Let $j_{M}:(M, \widehat{M}) \rightarrow \widehat{M}$ be the morphism given by the open embedding $M \subset \widehat{M}$. Then

(i) The functors $j_{M}^{-1} \simeq j_{M}^{!}: \mathrm{D}^{\mathrm{b}}\left(\mathrm{Ik}_{\widehat{M}}\right) \rightarrow \mathrm{D}^{\mathrm{b}}\left(\mathrm{Ik}_{(M, \widehat{M})}\right)$ are isomorphic to the quotient functor.

(ii) For $F \in \mathrm{D}^{\mathrm{b}}\left(\mathrm{Ik}_{\widehat{M}}\right)$ one has the isomorphisms in $\mathrm{D}^{\mathrm{b}}\left(\mathrm{Ik}_{\widehat{M}}\right)$

$$
\mathrm{R} j_{M ! !} j_{M}^{-1} F \simeq \mathbf{k}_{M} \otimes F, \quad \mathrm{R} j_{M *} j_{M}^{!} F \simeq \mathrm{R} \mathscr{I h o m}\left(\mathbf{k}_{M}, F\right) .
$$

(iii) The functors $\otimes$ and $\mathrm{R}$ Ihom commute with $j_{M}^{-1} \simeq j_{M}^{1}$.

(iv) The functor $\otimes$ commutes with $\mathrm{R} j_{M ! !}$ and the functor $\mathrm{R} \mathscr{I}$ hom commutes with $\mathrm{R} j_{M *}$.

The operations for indsheaves on bordered spaces satisfy similar properties as for usual spaces.

Lemma 5.2.5. Let $f:(M, \widehat{M}) \rightarrow(N, \widehat{N})$ and $g:(L, \widehat{L}) \rightarrow(M, \widehat{M})$ be morphisms of bordered spaces.

(i) The functor $\mathrm{R} f_{! !}$is left adjoint to $f^{!}$.

(ii) The functor $f^{-1}$ is left adjoint to $\mathrm{R} f_{*}$.

(iii) One has $\mathrm{R}(f \circ g)_{! !} \simeq \mathrm{R} f_{! !} \circ \mathrm{R} g_{! !}, \mathrm{R}(f \circ g)_{*} \simeq \mathrm{R} f_{*} \circ \mathrm{R} g_{*},(f \circ g)^{-1} \simeq$ $g^{-1} \circ f^{-1}$ and $(f \circ g)^{!} \simeq g^{!} \circ f^{!}$.

Corollary 5.2.6. If $f:(M, \widehat{M}) \rightarrow(N, \widehat{N})$ is an isomorphism of bordered spaces, then $\mathrm{R} f_{*} \simeq \mathrm{R} f_{! !}$and $f^{-1} \simeq f^{!}$. Moreover, $\mathrm{R} f_{*}$ and $f^{-1}$ are quasiinverse to each other. 
Most of the formulas for indsheaves on usual spaces extend to bordered spaces.

Proposition 5.2.7. Let $f:(M, \widehat{M}) \rightarrow(N, \widehat{N})$ be a morphism of bordered spaces. For $F \in \mathrm{D}^{\mathrm{b}}\left(\mathrm{I}_{(M, \widehat{M})}\right)$ and $G, G_{1}, G_{2} \in \mathrm{D}^{\mathrm{b}}\left(\mathrm{I}_{(N, \widehat{N})}\right)$, one has isomorphisms

$$
\begin{aligned}
\mathrm{R} f_{! !}\left(f^{-1} G \otimes F\right) & \simeq G \otimes \mathrm{R} f_{! !} F, \\
f^{-1}\left(G_{1} \otimes G_{2}\right) & \simeq f^{-1} G_{1} \otimes f^{-1} G_{2}, \\
\mathrm{R} \mathscr{I h o m}\left(G, \mathrm{R} f_{*} F\right) & \simeq \mathrm{R} f_{*} \mathrm{R} \mathscr{I h o m}\left(f^{-1} G, F\right), \\
\mathrm{R} \mathscr{I h o m}\left(\mathrm{R} f_{!} F, G\right) & \simeq \mathrm{R} f_{*} \operatorname{R} \mathscr{I h o m}\left(F, f^{!} G\right), \\
f^{!} \operatorname{R} \mathscr{I h o m}\left(G_{1}, G_{2}\right) & \simeq \operatorname{R} \mathscr{I h o m}\left(f^{-1} G_{1}, f^{!} G_{2}\right),
\end{aligned}
$$

and a morphism

$$
f^{-1} \mathrm{R} \mathscr{I h o m}\left(G_{1}, G_{2}\right) \rightarrow \mathrm{R} \mathscr{I h o m}\left(f^{-1} G_{1}, f^{-1} G_{2}\right) .
$$

Lemma 5.2.8. Consider a Cartesian diagram in the category of bordered spaces

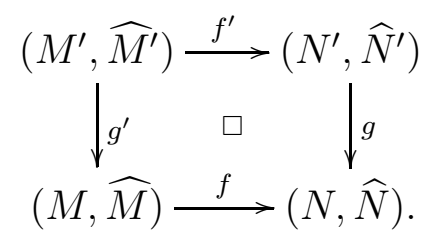

Then there are isomorphisms of functors $\mathrm{D}^{\mathrm{b}}\left(\mathrm{Ik}_{\left(M^{\prime}, \widehat{M^{\prime}}\right)}\right) \rightarrow \mathrm{D}^{\mathrm{b}}\left(\mathrm{Ik}_{(N, \widehat{N})}\right)$

$$
g^{-1} \mathrm{R} f_{! !} \simeq \mathrm{R} f_{! !}^{\prime} g^{\prime-1}, \quad g^{!} \mathrm{R} f_{*} \simeq \mathrm{R} f_{*}^{\prime} g^{! !} .
$$

The notion of proper morphisms of topological spaces is extended to the case of bordered spaces as follows.

Definition 5.2.9. The morphism of bordered spaces $f:(M, \widehat{M}) \rightarrow(N, \widehat{N})$ is proper if the following two conditions hold:

(a) $f: M \rightarrow N$ is proper,

(b) the projection $\bar{\Gamma}_{f} \rightarrow \widehat{N}$ is proper.

Lemma 5.2.10. The map $f:(M, \widehat{M}) \rightarrow(N, \widehat{N})$ is proper if and only if the following two conditions hold: 
(a) $\bar{\Gamma}_{f} \times_{\widehat{N}} N \subset \Gamma_{f}$.

(b) the projection $\bar{\Gamma}_{f} \rightarrow \widehat{N}$ is proper.

Proposition 5.2.11. Assume that $f:(M, \widehat{M}) \rightarrow(N, \widehat{N})$ is proper. Then $\mathrm{R} f_{! !} \stackrel{\sim}{\sim} \mathrm{R} f_{*}$ as functors $\mathrm{D}^{\mathrm{b}}\left(\mathrm{Ik}_{(M, \widehat{M})}\right) \rightarrow \mathrm{D}^{\mathrm{b}}\left(\mathrm{Ik}_{(N, \widehat{N})}\right)$.

\section{Enhanced indsheaves}

In this section, extracted from [DK13], one extends some constructions of Tamarkin [Ta 08] to indsheaves on bordered spaces. We refer to [GS 12] for a detailed exposition and some complements to Tamarkin's paper.

\subsection{Tamarkin's construction}

Let $M$ be a smooth manifold and denote by $T^{*} M$ its cotangent bundle. Given $F \in \mathrm{D}^{\mathrm{b}}\left(\mathbf{k}_{M}\right)$, its microsupport $S S(F) \subset T^{*} M$ (see [KS90]) describes the codirections of non propagation for the cohomology of $F$. It is a closed conic co-isotropic subset of $T^{*} M$.

In order to treat co-isotropic subsets of $T^{*} M$ which are not necessarily conic, Tamarkin adds a real variable $t \in \mathbb{R}$. Denoting by $\left(t, t^{*}\right)$ the symplectic coordinates of $T^{*} \mathbb{R}$, consider the full subcategory $\mathrm{D}_{t^{*} \leq 0}^{\mathrm{b}}\left(\mathbf{k}_{M \times \mathbb{R}}\right) \subset \mathrm{D}^{\mathrm{b}}\left(\mathbf{k}_{M \times \mathbb{R}}\right)$ whose objects $K$ satisfy $S S(K) \subset\left\{t^{*} \leq 0\right\}$. There are equivalences

$$
{ }^{\perp} \mathrm{D}_{t^{*} \leq 0}^{\mathrm{b}}\left(\mathbf{k}_{M \times \mathbb{R}}\right) \simeq \mathrm{D}^{\mathrm{b}}\left(\mathbf{k}_{M \times \mathbb{R}}\right) / \mathrm{D}_{t^{*} \leq 0}^{\mathrm{b}}\left(\mathbf{k}_{M \times \mathbb{R}}\right) \simeq \mathrm{D}_{t^{*} \leq 0}^{\mathrm{b}}\left(\mathbf{k}_{M \times \mathbb{R}}\right)^{\perp}
$$

between the quotient category and the left and right orthogonal categories.

Let us recall the description of the first equivalence.

For $K, L \in \mathrm{D}^{\mathrm{b}}\left(\mathbf{k}_{M \times \mathbb{R}}\right)$, consider the convolution functor with respect to the $t$ variable

$$
K \stackrel{+}{\otimes} L:=\mathrm{R} \mu_{!}\left(q_{1}^{-1} K \otimes q_{2}^{-1} L\right),
$$

where $\mu, q_{1} ; q_{2}: M \times \mathbb{R} \times \mathbb{R}$ are given by $\mu\left(x, t_{1}, t_{2}\right)=\left(x, t_{1}+t_{2}\right), q_{1}\left(x, t_{1}, t_{2}\right)=$ $\left(x, t_{1}\right)$ and $q_{2}\left(x, t_{1}, t_{2}\right)=\left(x, t_{2}\right)$.

One sets

$$
\mathbf{k}_{\{t \geq 0\}}=\mathbf{k}_{\{(x, t) \in M \times \mathbb{R} ; t \in \mathbb{R}, t \geq 0\}},
$$

and we use similar notation for $\mathbf{k}_{\{t=0\}}$. These are sheaves on $M \times \mathbb{R}$. 
Note that $\mathbf{k}_{\{t=0\}} \stackrel{+}{\otimes} K \simeq K$. Then

$$
\begin{aligned}
\mathrm{D}_{t^{*} \leq 0}^{\mathrm{b}}\left(\mathbf{k}_{M \times \mathbb{R}}\right) & =\left\{K \in \mathrm{D}^{\mathrm{b}}\left(\mathbf{k}_{M \times \mathbb{R}}\right) ; \mathbf{k}_{\{t \geq 0\}} \stackrel{+}{\otimes} K \simeq 0\right\}, \\
{ }^{\perp} \mathrm{D}_{t^{*} \leq 0}^{\mathrm{b}}\left(\mathbf{k}_{M \times \mathbb{R}}\right) & =\left\{K \in \mathrm{D}^{\mathrm{b}}\left(\mathbf{k}_{M \times \mathbb{R}}\right) ; \mathbf{k}_{\{t \geq 0\}} \stackrel{+}{\otimes} K \stackrel{\longrightarrow}{\longrightarrow} K,\right.
\end{aligned}
$$

and one has an equivalence

$$
\mathrm{D}^{\mathrm{b}}\left(\mathbf{k}_{M \times \mathbb{R}}\right) / \mathrm{D}_{t^{*} \leq 0}^{\mathrm{b}}\left(\mathbf{k}_{M \times \mathbb{R}}\right) \stackrel{\sim}{\longrightarrow}{ }^{\perp} \mathrm{D}_{t^{*} \leq 0}^{\mathrm{b}}\left(\mathbf{k}_{M \times \mathbb{R}}\right), \quad K \mapsto \mathbf{k}_{\{t \geq 0\}} \stackrel{+}{\otimes} K .
$$

We will adapt this construction to the case of indsheaves and a good topological space $M$ in the sequel.

\subsection{Convolution products}

Consider the 2-point compactification of the real line $\overline{\mathbb{R}}:=\mathbb{R} \sqcup\{+\infty,-\infty\}$. Denote by $\mathbb{P}^{1}(\mathbb{R})=\mathbb{R} \sqcup\{\infty\}$ the real projective line. Then $\overline{\mathbb{R}}$ has a structure of subanalytic space such that the natural map $\overline{\mathbb{R}} \rightarrow \mathbb{P}^{1}(\mathbb{R})$ is a subanalytic map.

Notation 6.2.1. We will consider the bordered space

$$
\mathbb{R}_{\infty}:=(\mathbb{R}, \overline{\mathbb{R}}) .
$$

Note that $\mathbb{R}_{\infty}$ is isomorphic to $\left(\mathbb{R}, \mathbb{P}^{1}(\mathbb{R})\right)$ as a bordered space.

Consider the morphisms of bordered spaces

$$
\begin{gathered}
a: \mathbb{R}_{\infty} \rightarrow \mathbb{R}_{\infty}, \\
\mu, q_{1}, q_{2}: \mathbb{R}_{\infty} \times \mathbb{R}_{\infty} \rightarrow \mathbb{R}_{\infty},
\end{gathered}
$$

where $a(t)=-t, \mu\left(t_{1}, t_{2}\right)=t_{1}+t_{2}$ and $q_{1}, q_{2}$ are the natural projections.

For a good topological space $M$, we will use the same notations for the associated morphisms

$$
\begin{gathered}
a: M \times \mathbb{R}_{\infty} \rightarrow M \times \mathbb{R}_{\infty}, \\
\mu, q_{1}, q_{2}: M \times \mathbb{R}_{\infty} \times \mathbb{R}_{\infty} \rightarrow M \times \mathbb{R}_{\infty} .
\end{gathered}
$$

We also use the natural morphisms

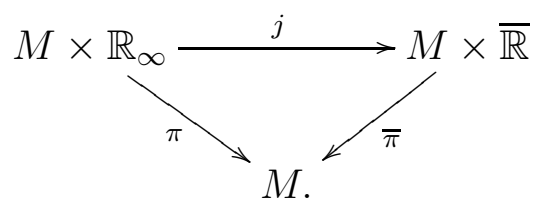


Notation 6.2.2. We sometimes write $\mathrm{D}^{\mathrm{b}}\left(\mathbf{k}_{M \times \mathbb{R}_{\infty}}\right)$ for $\mathrm{D}^{\mathrm{b}}\left(\mathbf{k}_{M \times \mathbb{R}}\right)$ regarded as a full subcategory $\mathrm{D}^{\mathrm{b}}\left(\mathrm{Ik}_{M \times \mathbb{R}_{\infty}}\right)$.

Definition 6.2.3. The functors

$$
\begin{gathered}
\stackrel{+}{\otimes}: \mathrm{D}^{\mathrm{b}}\left(\mathrm{Ik}_{M \times \mathbb{R}_{\infty}}\right) \times \mathrm{D}^{\mathrm{b}}\left(\mathrm{Ik}_{M \times \mathbb{R}_{\infty}}\right) \rightarrow \mathrm{D}^{\mathrm{b}}\left(\mathrm{Ik}_{M \times \mathbb{R}_{\infty}}\right), \\
\mathscr{I} \mathrm{Iom}^{+}: \mathrm{D}^{-}\left(\mathrm{Ik}_{M \times \mathbb{R}_{\infty}}\right)^{\mathrm{op}} \times \mathrm{D}^{+}\left(\mathrm{Ik}_{M \times \mathbb{R}_{\infty}}\right) \rightarrow \mathrm{D}^{+}\left(\mathrm{Ik}_{M \times \mathbb{R}_{\infty}}\right),
\end{gathered}
$$

are defined by

$$
\begin{aligned}
K_{1} \stackrel{+}{\otimes} K_{2} & =\mathrm{R} \mu_{! !}\left(q_{1}^{-1} K_{1} \otimes q_{2}^{-1} K_{2}\right), \\
\mathscr{I h o m}^{+}\left(K_{1}, K_{2}\right) & =\mathrm{R} q_{1 *} \operatorname{R} \mathscr{I h o m}\left(q_{2}^{-1} K_{1}, \mu^{!} K_{2}\right) .
\end{aligned}
$$

Although we work now on $M \times \overline{\mathbb{R}}$, we keep the same notations as in (6.1.1) and one sets

$$
\mathbf{k}_{\{t \geq 0\}}=\mathbf{k}_{\{(x, t) \in M \times \overline{\mathbb{R}} ; t \in \mathbb{R}, t \geq 0\}} .
$$

We use similar notation for $\mathbf{k}_{\{t=0\}}, \mathbf{k}_{\{t>0\}}, \mathbf{k}_{\{t \leq 0\}}, \mathbf{k}_{\{t<0\}}, \mathbf{k}_{\{t \neq 0\}}$ and $\mathbf{k}_{\{t=a\}}$, etc. These are sheaves on $M \times \overline{\mathbb{R}}$ whose stalk vanishes at points of $M \times(\overline{\mathbb{R}} \backslash \mathbb{R})$. We also regard them as objects of $\mathrm{D}^{\mathrm{b}}\left(\mathrm{Ik}_{M \times \mathbb{R}_{\infty}}\right)$.

Lemma 6.2.4. For $K \in \mathrm{D}^{\mathrm{b}}\left(\mathrm{I}_{M \times \mathbb{R}_{\infty}}\right)$ there are isomorphisms

$$
\mathbf{k}_{\{t=0\}} \stackrel{+}{\otimes} K \simeq K \simeq \mathscr{I} \operatorname{hom}^{+}\left(\mathbf{k}_{\{t=0\}}, K\right) .
$$

More generally, for $a \in \mathbb{R}$, we have

$$
\mathbf{k}_{\{t=a\}} \stackrel{+}{\otimes} K \simeq \mathrm{R} \mu_{a *} K \simeq \mathscr{I} \operatorname{hom}^{+}\left(\mathbf{k}_{\{t=-a\}}, K\right),
$$

where $\mu_{a}: M \times \mathbb{R}_{\infty} \rightarrow M \times \mathbb{R}_{\infty}$ is the morphism induced by the translation $t \mapsto t+a$.

Corollary 6.2.5. The category $\mathrm{D}^{\mathrm{b}}\left(\mathrm{Ik}_{M \times \mathbb{R}_{\infty}}\right)$ has a structure of commutative tensor category with $\stackrel{+}{\otimes}$ as tensor product and $\mathbf{k}_{\{t=0\}}$ as unit object.

As seen in (6.2.4) below, the functor $\mathscr{I h o m}^{+}$is the inner hom of the tensor category $\mathrm{D}^{\mathrm{b}}\left(\mathrm{Ik}_{M \times \mathbb{R}_{\infty}}\right)$. 
Lemma 6.2.6. For $K_{1}, K_{2}, K_{3} \in \mathrm{D}^{\mathrm{b}}\left(\mathrm{I}_{M \times \mathbb{R}_{\infty}}\right)$ one has

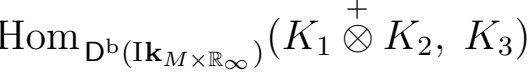

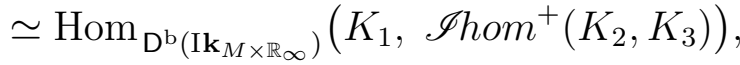

$$
\begin{aligned}
& \mathscr{I h o m}^{+}\left(K_{1} \stackrel{+}{\otimes} K_{2}, K_{3}\right) \simeq \mathscr{I h o m}^{+}\left(K_{1}, \mathscr{I h o m}^{+}\left(K_{2}, K_{3}\right)\right) \text {, } \\
& \mathrm{R} \pi_{*} \mathrm{R} \mathscr{I h o m}\left(K_{1} \stackrel{+}{\otimes} K_{2}, K_{3}\right) \simeq \mathrm{R} \pi_{*} \operatorname{R} \mathscr{I h o m}\left(K_{1}, \mathscr{I h o m}^{+}\left(K_{2}, K_{3}\right)\right) .
\end{aligned}
$$

The following lemmas are used to define the category of enhanced indsheaves.

Lemma 6.2.7. For $K_{1}, K_{2} \in \mathrm{D}^{\mathrm{b}}\left(\mathrm{Ik}_{M \times \mathbb{R}_{\infty}}\right)$ and $L \in \mathrm{D}^{\mathrm{b}}\left(\mathrm{Ik}_{M}\right)$ one has

$$
\begin{aligned}
\pi^{-1} L \otimes\left(K_{1} \stackrel{+}{\otimes} K_{2}\right) & \simeq\left(\pi^{-1} L \otimes K_{1}\right) \stackrel{+}{\otimes} K_{2}, \\
\operatorname{R} \mathscr{I h o m}\left(\pi^{-1} L, \mathscr{I h o m}\left(K_{1}, K_{2}\right)\right) & \simeq \mathscr{I h o m}\left(\pi^{-1} L \otimes K_{1}, K_{2}\right) \\
& \simeq \mathscr{I h o m}{ }^{+}\left(K_{1}, \operatorname{R} \mathscr{I h o m}\left(\pi^{-1} L, K_{2}\right)\right) .
\end{aligned}
$$

Lemma 6.2.8. For $K \in \mathrm{D}^{\mathrm{b}}\left(\mathrm{I}_{M \times \mathbb{R}_{\infty}}\right)$ and $L \in \mathrm{D}^{\mathrm{b}}\left(\mathrm{Ik}_{M}\right)$ one has

$$
\begin{aligned}
\pi^{-1} L \otimes K & \simeq\left(\pi^{-1} L \otimes \mathbf{k}_{\{t=0\}}\right) \stackrel{+}{\otimes} K, \\
\mathrm{R} \mathscr{I h o m ~}\left(\pi^{-1} L, K\right) & \simeq \mathscr{I h o m}^{+}\left(\pi^{-1} L \otimes \mathbf{k}_{\{t=0\}}, K\right), \\
a^{-1} \mathrm{R} \mathscr{I h o m}\left(K, \pi^{!} L\right) & \simeq \mathscr{I h o m}^{+}\left(K, \mathbf{k}_{\{t=0\}} \otimes \pi^{-1} L\right) .
\end{aligned}
$$

Here $a$ is the involution of $M \times \mathbb{R}_{\infty}$ given by $(x, t) \mapsto(x,-t)$.

Lemma 6.2.9. For $K_{1}, K_{2} \in \mathrm{D}^{\mathrm{b}}\left(\mathrm{Ik}_{M \times \mathbb{R}_{\infty}}\right)$ there are isomorphisms

$$
\begin{aligned}
\mathrm{R} \pi_{! !}\left(K_{1} \stackrel{+}{\otimes} K_{2}\right) & \simeq \mathrm{R} \pi_{! !} K_{1} \otimes \mathrm{R} \pi_{! !} K_{2}, \\
\mathrm{R} \pi_{*} \mathscr{I} \text { hom }^{+}\left(K_{1}, K_{2}\right) & \simeq \mathrm{R} \mathscr{I h o m}\left(\mathrm{R} \pi_{! !} K_{1}, \mathrm{R} \pi_{*} K_{2}\right) .
\end{aligned}
$$

Corollary 6.2.10. For any $K \in \mathrm{D}^{\mathrm{b}}\left(\mathrm{I}_{M \times \mathbb{R}_{\infty}}\right)$, one has

$$
\begin{aligned}
& \mathrm{R} \pi_{! !}\left(\mathbf{k}_{t \geq 0} \stackrel{+}{\otimes} K\right) \simeq 0, \\
& \mathrm{R} \pi_{*} \mathscr{I h o m}{ }^{+}\left(\mathbf{k}_{t \geq 0}, K\right) \simeq 0 .
\end{aligned}
$$


Lemma 6.2.11. For $K \in \mathrm{D}^{\mathrm{b}}\left(\mathrm{Ik}_{M \times \mathbb{R}_{\infty}}\right)$ and $L \in \mathrm{D}^{\mathrm{b}}\left(\mathrm{Ik}_{M}\right)$ one has

$$
\begin{aligned}
\left(\pi^{-1} L\right) \stackrel{+}{\otimes} K & \simeq \pi^{-1}\left(L \otimes \mathrm{R} \pi_{! !} K\right), \\
\mathscr{I h o m}^{+}\left(\pi^{-1} L, K\right) & \simeq \pi^{!} \mathrm{R} \mathscr{I h o m}\left(L, \mathrm{R} \pi_{*} K\right), \\
\mathscr{I h o m}^{+}\left(K, \pi^{!} L\right) & \simeq \pi^{!} \mathrm{R} \mathscr{I h o m}\left(\mathrm{R} \pi_{! !} K, L\right) .
\end{aligned}
$$

Proposition 6.2.12. For $K \in \mathrm{D}^{\mathrm{b}}\left(\mathrm{Ik}_{M \times \mathbb{R}_{\infty}}\right)$, one has a distinguished triangle

$$
\pi^{-1} L \longrightarrow \mathbf{k}_{\{t \geq 0\}} \stackrel{+}{\otimes} K \longrightarrow \mathscr{I} \operatorname{hom}^{+}\left(\mathbf{k}_{\{t \geq 0\}}, K\right) \stackrel{+1}{\longrightarrow}
$$

with $L=\mathrm{R} \pi_{*}\left(\mathbf{k}_{\{t \geq 0\}} \stackrel{+}{\otimes} K\right) \simeq \mathrm{R} \pi_{! !} \mathscr{I} h^{+}{ }^{+}\left(\mathbf{k}_{\{t \geq 0\}}, K\right)$.

\subsection{Enhanced indsheaves}

Definition 6.3.1. Consider the full triangulated subcategories of $\mathrm{D}^{\mathrm{b}}\left(\mathrm{Ik}_{M \times \mathbb{R}_{\infty}}\right)$

$$
\begin{aligned}
\mathrm{IC}_{t^{*} \leq 0} & =\left\{K ; \mathbf{k}_{\{t \geq 0\}} \stackrel{+}{\otimes} K \simeq 0\right\} \\
& =\left\{K ; \mathscr{I h o m}^{+}\left(\mathbf{k}_{\{t \geq 0\}}, K\right) \simeq 0\right\}, \\
\mathrm{IC}_{t^{*} \geq 0} & =\left\{K ; \mathbf{k}_{\{t \leq 0\}} \stackrel{+}{\otimes} K \simeq 0\right\} \\
& =\left\{K ; \mathscr{I} \operatorname{Iom}^{+}\left(\mathbf{k}_{\{t \leq 0\}}, K\right) \simeq 0\right\}, \\
\mathrm{IC}_{t^{*}=0} & =\mathrm{IC}_{t^{*} \leq 0} \cap \mathrm{IC}_{t^{*} \geq 0} .
\end{aligned}
$$

Consider also the corresponding quotient categories

$$
\begin{aligned}
\mathrm{E}_{ \pm}^{\mathrm{b}}\left(\mathrm{I}_{M}\right) & =\mathrm{IC}_{ \pm t^{*} \geq 0} / \mathrm{IC}_{t^{*}=0} \\
\mathrm{E}^{\mathrm{b}}\left(\mathrm{Ik}_{M}\right) & =\mathrm{D}^{\mathrm{b}}\left(\mathrm{I}_{M \times \mathbb{R}_{\infty}}\right) / \mathrm{IC}_{t^{*}=0}
\end{aligned}
$$

They are triangulated categories. One calls $\mathrm{E}^{\mathrm{b}}\left(\mathrm{Ik}_{M}\right)$ the triangulated category of enhanced indsheaves. One defines similarly the categories $\mathrm{E}\left(\mathrm{Ik}_{M}\right)$, $\mathrm{E}^{+}\left(\mathrm{Ik}_{M}\right)$ and $\mathrm{E}^{-}\left(\mathrm{Ik}_{M}\right)$.

Notice that

$$
\begin{aligned}
\mathrm{IC}_{t^{*}=0} & =\left\{K \in \mathrm{D}^{\mathrm{b}}\left(\mathrm{Ik}_{M \times \mathbb{R}_{\infty}}\right) ; \pi^{-1} \mathrm{R} \pi_{*} K \stackrel{\longrightarrow}{\longrightarrow} K\right\} \\
& =\left\{K \in \mathrm{D}^{\mathrm{b}}\left(\mathrm{Ik}_{M \times \mathbb{R}_{\infty}}\right) ; K \sim \pi^{!} \mathrm{R} \pi_{! !} K\right\} \\
& =\left\{K \in \mathrm{D}^{\mathrm{b}}\left(\mathrm{Ik}_{M \times \mathbb{R}_{\infty}}\right) ; K \simeq \pi^{-1} L \text { for some } L \in \mathrm{D}^{\mathrm{b}}\left(\mathrm{Ik}_{M}\right)\right\}
\end{aligned}
$$


Therefore,

$$
\mathrm{E}^{\mathrm{b}}\left(\mathrm{Ik}_{M}\right) \simeq \mathrm{D}^{\mathrm{b}}\left(\mathrm{Ik}_{M \times \mathbb{R}_{\infty}}\right) /\left\{K \in \mathrm{D}^{\mathrm{b}}\left(\mathrm{Ik}_{M \times \mathbb{R}_{\infty}}\right) ; \pi^{-1} \mathrm{R} \pi_{*} K \stackrel{\sim}{\longrightarrow} K\right\}
$$

One also defines the category $\mathrm{E}^{\mathrm{b}}\left(\mathbf{k}_{M}\right)$ as

$$
\mathrm{E}^{\mathrm{b}}\left(\mathbf{k}_{M}\right)=\mathrm{D}^{\mathrm{b}}\left(\mathbf{k}_{M \times \mathbb{R}}\right) /\left\{K ; \pi^{-1} \mathrm{R} \pi_{*} K \stackrel{\sim}{\longrightarrow} K\right\}
$$

Then $\mathrm{E}^{\mathrm{b}}\left(\mathbf{k}_{M}\right)$ is a full subcategory of $\mathrm{E}^{\mathrm{b}}\left(\mathrm{Ik}_{M}\right)$.

Proposition 6.3.2. There are equivalences of triangulated categories

$$
\begin{aligned}
& \mathrm{E}_{ \pm}^{\mathrm{b}}\left(\mathrm{I}_{M}\right) \simeq \mathrm{D}^{\mathrm{b}}\left(\mathrm{I}_{M \times \mathbb{R}_{\infty}}\right) / \mathrm{IC}_{ \pm t^{*} \leq 0}, \\
& \mathrm{E}^{\mathrm{b}}\left(\mathrm{Ik}_{M}\right) \simeq \mathrm{E}_{+}^{\mathrm{b}}\left(\mathrm{Ik}_{M}\right) \oplus \mathrm{E}_{-}^{\mathrm{b}}\left(\mathrm{Ik}_{M}\right) .
\end{aligned}
$$

This follows from Proposition 6.3.4 below.

Remark 6.3.3. The categories $\mathrm{E}_{+}^{\mathrm{b}}\left(\mathrm{Ik}_{M}\right)$ are the analogue of Tamarkin's construction in the framework of indsheaves.

Proposition 6.3.4. One has

$$
\begin{aligned}
& { }^{\perp} \mathrm{IC}_{ \pm t^{*} \leq 0}=\left\{K ; \mathbf{k}_{\{ \pm t \geq 0\}} \stackrel{+}{\otimes} K \stackrel{\sim}{\longrightarrow} K\right\}=\left\{K ; \mathbf{k}_{\{ \pm t>0\}} \stackrel{+}{\otimes} K \simeq 0\right\}, \\
& \mathrm{IC}_{ \pm t^{*} \leq 0}^{\perp}=\left\{K ; K \stackrel{\sim}{\longrightarrow} \mathscr{I h o m}^{+}\left(\mathbf{k}_{\{ \pm t \geq 0\}}, K\right)\right\} \\
& =\left\{K ; \mathscr{I h o m}^{+}\left(\mathbf{k}_{\{ \pm t>0\}}, K\right) \simeq 0\right\} \text {, } \\
& { }^{\perp} \mathrm{IC}_{t^{*}=0}=\left\{K ;\left(\mathbf{k}_{\{t \geq 0\}} \oplus \mathbf{k}_{\{t \leq 0\}}\right) \stackrel{+}{\otimes} K \stackrel{\sim}{\longrightarrow} K\right\} \\
& =\left\{K ; \mathbf{k}_{M \times \mathbb{R}} \stackrel{+}{\otimes} K \simeq 0\right\}=\left\{K ; \mathrm{R} \pi_{! !} K \simeq 0\right\}, \\
& \mathrm{IC}_{t^{*}=0}^{\perp}=\left\{K ; \mathscr{I h o m}{ }^{+}\left(\mathbf{k}_{\{t \geq 0\}} \oplus \mathbf{k}_{\{t \leq 0\}}, K\right) \stackrel{\sim}{\longrightarrow} K\right\} \\
& =\left\{K ; \mathscr{I h o m}^{+}\left(\mathbf{k}_{M \times \mathbb{R}}, K\right) \simeq 0\right\}=\left\{K ; \mathrm{R} \pi_{*} K \simeq 0\right\}, \\
& { }^{\perp} \mathrm{IC}_{t^{*}=0}={ }^{\perp} \mathrm{IC}_{t^{*} \geq 0} \oplus{ }^{\perp} \mathrm{IC}_{t^{*} \leq 0}, \\
& \mathrm{IC}_{t^{*}=0}^{\perp}=\mathrm{IC}_{t^{*} \geq 0}^{\perp} \oplus \mathrm{IC}_{t^{*} \leq 0}^{\perp} .
\end{aligned}
$$

Moreover, one has the equivalences

$$
\begin{aligned}
& \mathrm{E}_{ \pm}^{\mathrm{b}}\left(\mathrm{Ik}_{M}\right) \stackrel{\sim}{\sim}{ }^{\perp} \mathrm{IC}_{ \pm t^{*} \leq 0}, \quad K \mapsto \mathbf{k}_{\{ \pm t \geq 0\}} \stackrel{+}{\otimes} K, \\
& \mathrm{E}_{ \pm}^{\mathrm{b}}\left(\mathrm{Ik}_{M}\right) \stackrel{\sim}{\longrightarrow} \mathrm{IC}_{ \pm t^{*} \leq 0}^{\perp}, \quad K \mapsto \mathscr{I} \operatorname{hom}^{+}\left(\mathbf{k}_{\{ \pm t \geq 0\}}, K\right), \\
& \mathrm{E}^{\mathrm{b}}\left(\mathrm{Ik}_{M}\right) \stackrel{\sim}{\longrightarrow}{ }^{\perp} \mathrm{IC}_{t^{*}=0}, \quad K \mapsto\left(\mathbf{k}_{\{t \geq 0\}} \oplus \mathbf{k}_{\{t \leq 0\}}\right) \stackrel{+}{\otimes} K, \\
& \mathrm{E}^{\mathrm{b}}\left(\mathrm{Ik}_{M}\right) \stackrel{\sim}{\longrightarrow} \mathrm{IC}_{t^{*}=0}^{\perp}, \quad K \mapsto \mathscr{I h o m}{ }^{+}\left(\mathbf{k}_{\{t \geq 0\}} \oplus \mathbf{k}_{\{t \leq 0\}}, K\right),
\end{aligned}
$$

where the quasi-inverse functors are given by the quotient functors. 
These categories are illustrated as follows:

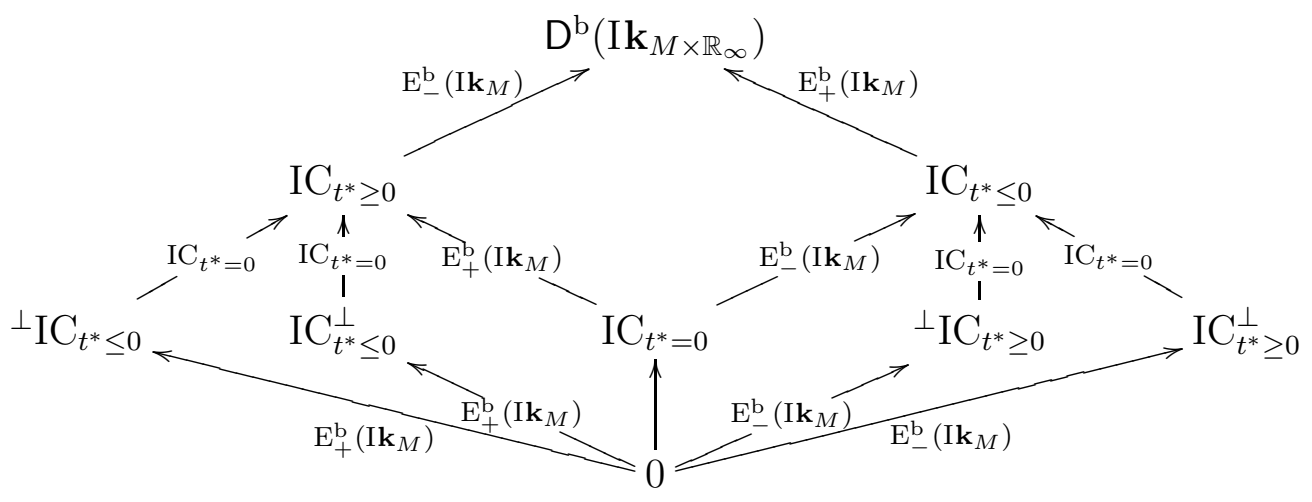

Here, $A \longrightarrow C \rightarrow B$ or $A \underset{C}{\longrightarrow} B$ means that $C \simeq B / A$.

Definition 6.3.5. One introduces the functors

$$
\begin{aligned}
& \mathrm{L}^{\mathrm{E}}=\left(\mathbf{k}_{\{t \geq 0\}} \oplus \mathbf{k}_{\{t \leq 0\}}\right) \stackrel{+}{\otimes}(\bullet), \quad \mathrm{E}^{\mathrm{b}}\left(\mathrm{Ik}_{M}\right) \rightarrow{ }^{\perp} \mathrm{IC}_{t^{*}=0} \subset \mathrm{D}^{\mathrm{b}}\left(\mathrm{Ik}_{M \times \mathbb{R}_{\infty}}\right), \\
& \mathrm{R}^{\mathrm{E}}=\mathscr{I h o m}^{+}\left(\mathbf{k}_{\{t \geq 0\}} \oplus \mathbf{k}_{\{t \leq 0\}}, \bullet\right), \quad \mathrm{E}^{\mathrm{b}}\left(\mathrm{Ik}_{M}\right) \rightarrow \mathrm{IC}_{t^{*}=0}^{\perp} \subset \mathrm{D}^{\mathrm{b}}\left(\mathrm{Ik}_{M \times \mathbb{R}_{\infty}}\right) .
\end{aligned}
$$

The functors $\mathrm{L}^{\mathrm{E}}$ and $\mathrm{R}^{\mathrm{E}}$ are the left and right adjoint of the quotient functor $\mathrm{D}^{\mathrm{b}}\left(\mathrm{Ik}_{M \times \mathbb{R}_{\infty}}\right) \rightarrow \mathrm{E}^{\mathrm{b}}\left(\mathrm{Ik}_{M}\right)$, and the two compositions

$$
\mathrm{E}^{\mathrm{b}}\left(\mathrm{I}_{M}\right) \underset{\mathrm{R}^{\mathrm{E}}}{\stackrel{\mathrm{L}^{\mathrm{E}}}{\longrightarrow}} \mathrm{D}^{\mathrm{b}}\left(\mathrm{I} \mathbf{k}_{M \times \mathbb{R}_{\infty}}\right) \longrightarrow \mathrm{E}^{\mathrm{b}}\left(\mathrm{I}_{M}\right)
$$

are isomorphic to the identity.

Definition 6.3.6. One defines the hom functor

$$
\begin{aligned}
\mathscr{I h o m}^{\mathrm{E}}: \mathrm{E}^{\mathrm{b}}\left(\mathrm{Ik}_{M}\right)^{\mathrm{op}} \times \mathrm{E}^{\mathrm{b}}\left(\mathrm{Ik}_{M}\right) & \rightarrow \mathrm{D}^{+}\left(\mathrm{Ik}_{M}\right) \\
\mathscr{I h o m}{ }^{\mathrm{E}}\left(K_{1}, K_{2}\right) & =\mathrm{R} \pi_{*} \mathrm{R} \mathscr{I h o m}\left(\mathrm{L}^{\mathrm{E}}\left(K_{1}\right), \mathrm{R}^{\mathrm{E}}\left(K_{2}\right)\right),
\end{aligned}
$$

and one sets

$$
\begin{aligned}
& \mathscr{H} o m^{\mathrm{E}}=\alpha_{M} \circ \mathscr{I h o m}{ }^{\mathrm{E}}: \mathrm{E}^{\mathrm{b}}\left(\mathrm{Ik}_{M}\right)^{\mathrm{op}} \times \mathrm{E}^{\mathrm{b}}\left(\mathrm{Ik}_{M}\right) \rightarrow \mathrm{D}^{+}\left(\mathbf{k}_{M}\right), \\
& \operatorname{RHom}^{\mathrm{E}}\left(K_{1}, K_{2}\right)=\operatorname{R} \Gamma\left(M ; \mathscr{H} o m^{\mathrm{E}}\left(K_{1}, K_{2}\right)\right) \in \mathrm{D}^{\mathrm{b}}(\mathbf{k}) .
\end{aligned}
$$


Note that

$$
\begin{aligned}
\mathscr{I h o m}^{\mathrm{E}}\left(K_{1}, K_{2}\right) & \simeq \mathrm{R} \pi_{*} \operatorname{R} \mathscr{I h o m}\left(\mathrm{L}^{\mathrm{E}}\left(K_{1}\right), \mathrm{L}^{\mathrm{E}}\left(K_{2}\right)\right) \\
& \simeq \mathrm{R} \pi_{*} \mathrm{R} \mathscr{I} \text { hom }\left(\mathrm{R}^{\mathrm{E}}\left(K_{1}\right), \mathrm{R}^{\mathrm{E}}\left(K_{2}\right)\right)
\end{aligned}
$$

and

$$
\operatorname{Hom}_{\mathrm{E}^{\mathrm{b}}\left(\mathrm{Ik}_{M}\right)}\left(K_{1}, K_{2}\right) \simeq H^{0}\left(\operatorname{RHom}^{\mathrm{E}}\left(K_{1}, K_{2}\right)\right)
$$

\subsection{Operations on enhanced indsheaves}

By Lemma 6.2.11 the following definition is well posed.

Definition 6.4.1. The bifunctors

$$
\begin{aligned}
& \stackrel{+}{\otimes}: \mathrm{E}^{\mathrm{b}}\left(\mathrm{Ik}_{M}\right) \times \mathrm{E}^{\mathrm{b}}\left(\mathrm{Ik}_{M}\right) \rightarrow \mathrm{E}^{\mathrm{b}}\left(\mathrm{Ik}_{M}\right), \\
& \mathscr{I h o m}^{+}: \mathrm{E}^{-}\left(\mathrm{Ik}_{M}\right)^{\mathrm{op}} \times \mathrm{E}^{+}\left(\mathrm{Ik}_{M}\right) \rightarrow \mathrm{E}^{+}\left(\mathrm{Ik}_{M}\right)
\end{aligned}
$$

are those induced by the bifunctors $\stackrel{+}{\otimes}$ and $\mathscr{I} h o m^{+}$defined on $\mathrm{D}^{\mathrm{b}}\left(\mathrm{Ik}_{M \times \mathbb{R}_{\infty}}\right)$.

For any $K \in \mathrm{E}^{\mathrm{b}}\left(\mathrm{Ik}_{M}\right)$ there is an isomorphism in $\mathrm{E}^{\mathrm{b}}\left(\mathrm{Ik}_{M}\right)$

$$
\mathbf{k}_{\{t \geq 0\}} \stackrel{+}{\otimes} \underset{\longrightarrow}{\longrightarrow} \mathscr{I h o m}^{+}\left(\mathbf{k}_{\{t \geq 0\}}, K\right),
$$

which follows from Proposition 6.2.12.

The bifunctor $\stackrel{+}{\otimes}$ gives $\mathrm{E}^{\mathrm{b}}\left(\mathrm{Ik}_{M}\right)$ a structure of a commutative tensor category with $\mathbf{k}_{\{t=0\}}$ as a unit object. Note that

$$
\begin{aligned}
& \mathrm{L}^{\mathrm{E}}\left(\mathbf{k}_{\{t=0\}}\right) \simeq \mathbf{k}_{t \geq 0} \bigoplus \mathbf{k}_{t \leq 0}, \\
& \mathrm{R}^{\mathrm{E}}\left(\mathbf{k}_{\{t=0\}}\right) \simeq \mathbf{k}_{t<0}[1] \bigoplus \mathbf{k}_{t>0}[1] .
\end{aligned}
$$

Moreover, $\mathscr{I} \mathrm{hom}^{+}$is the inner hom of the tensor category $\mathrm{E}^{\mathrm{b}}\left(\mathrm{Ik}_{M}\right)$ :

Lemma 6.4.2. For $K_{1}, K_{2}, K_{3} \in \mathrm{E}^{\mathrm{b}}\left(\mathrm{Ik}_{M}\right)$ there is an isomorphism

$$
\operatorname{Hom}_{\mathrm{E}^{+}\left(\mathrm{Ik}_{M}\right)}\left(K_{1} \stackrel{+}{\otimes} K_{2}, K_{3}\right) \simeq \operatorname{Hom}_{\mathrm{E}^{+}\left(\mathrm{Ik}_{M}\right)}\left(K_{1}, \mathscr{I h o m}^{+}\left(K_{2}, K_{3}\right)\right) .
$$


We have the following orthogonal relations:

$$
\begin{aligned}
& \mathrm{E}_{+}^{\mathrm{b}}\left(\mathrm{Ik}_{M}\right) \stackrel{+}{\otimes} \mathrm{E}_{-}^{\mathrm{b}}\left(\mathrm{Ik}_{M}\right) \simeq 0, \\
& \mathscr{I h o m}\left(\mathrm{E}_{ \pm}^{\mathrm{b}}\left(\mathrm{Ik}_{M}\right), \mathrm{E}_{\mp}^{\mathrm{b}}\left(\mathrm{Ik}_{M}\right)\right) \simeq 0 .
\end{aligned}
$$

Definition 6.4.3. By Lemma 6.2.11 one gets functors

$$
\begin{gathered}
\pi^{-1}(\bullet) \otimes(\bullet): \mathrm{D}^{\mathrm{b}}\left(\mathrm{Ik}_{M}\right) \times \mathrm{E}^{\mathrm{b}}\left(\mathrm{Ik}_{M}\right) \rightarrow \mathrm{E}^{\mathrm{b}}\left(\mathrm{Ik}_{M}\right), \\
\mathrm{R} \mathscr{I h o m}\left(\pi^{-1}(\bullet), \bullet\right): \mathrm{D}^{-}\left(\mathrm{Ik}_{M}\right)^{\mathrm{op}} \times \mathrm{E}^{+}\left(\mathrm{Ik}_{M}\right) \rightarrow \mathrm{E}^{+}\left(\mathrm{Ik}_{M}\right) .
\end{gathered}
$$

Remark 6.4.4. The functor $\otimes$ does not factor through $\mathrm{E}^{\mathrm{b}}\left(\mathrm{Ik}_{M}\right) \times \mathrm{E}^{\mathrm{b}}\left(\mathrm{Ik}_{M}\right)$,

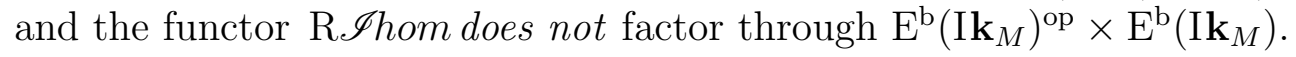

Let $f: M \rightarrow N$ be a continuous map of good topological spaces. Denote by $\tilde{f}: M \times \mathbb{R}_{\infty} \rightarrow N \times \mathbb{R}_{\infty}$ the associated morphism of bordered spaces. Then the composition of functors

$$
\begin{aligned}
\mathrm{R} \tilde{f}_{! !}, \mathrm{R} \tilde{f}_{*}: \mathrm{D}^{\mathrm{b}}\left(\mathrm{I}_{M \times \mathbb{R}_{\infty}}\right) & \rightarrow \mathrm{D}^{\mathrm{b}}\left(\mathrm{I}_{N \times \mathbb{R}_{\infty}}\right) \rightarrow \mathrm{E}^{\mathrm{b}}\left(\mathrm{Ik}_{N}\right), \\
\tilde{f}^{-1}, \tilde{f}^{!}: \mathrm{D}^{\mathrm{b}}\left(\mathrm{Ik}_{N \times \mathbb{R}_{\infty}}\right) & \rightarrow \mathrm{D}^{\mathrm{b}}\left(\mathrm{I}_{M \times \mathbb{R}_{\infty}}\right) \rightarrow \mathrm{E}^{\mathrm{b}}\left(\mathrm{Ik}_{M}\right),
\end{aligned}
$$

factor through $\mathrm{E}^{\mathrm{b}}\left(\mathrm{Ik}_{M}\right)$ and $\mathrm{E}^{\mathrm{b}}\left(\mathrm{Ik}_{N}\right)$, respectively.

Definition 6.4.5. One denotes by

$$
\begin{aligned}
& \mathrm{E} f_{! !}, \mathrm{E} f_{*}: \mathrm{E}^{\mathrm{b}}\left(\mathrm{Ik}_{M}\right) \rightarrow \mathrm{E}^{\mathrm{b}}\left(\mathrm{Ik}_{N}\right), \\
& \mathrm{E} f^{-1}, \mathrm{E} f^{!}: \mathrm{E}^{\mathrm{b}}\left(\mathrm{Ik}_{N}\right) \rightarrow \mathrm{E}^{\mathrm{b}}\left(\mathrm{Ik}_{M}\right),
\end{aligned}
$$

the functors induced by (6.4.1) and (6.4.2), respectively.

Definition 6.4.6. For $K \in \mathrm{E}^{\mathrm{b}}\left(\mathrm{Ik}_{M}\right)$ and $L \in \mathrm{E}^{\mathrm{b}}\left(\mathrm{Ik}_{N}\right)$, we define their external tensor product by

$$
K \stackrel{+}{\otimes} L=\mathrm{E} p_{1}^{-1} K \stackrel{+}{\otimes} \mathrm{E} p_{2}^{-1} L \in \mathrm{E}^{\mathrm{b}}\left(\mathrm{Ik}_{M \times N}\right),
$$

where $p_{1}$ and $p_{2}$ denote the projections from $M \times N$ to $M$ and $N$, respectively.

Using Definition 6.3.5, for $F \in \mathrm{E}^{\mathrm{b}}\left(\mathrm{Ik}_{M}\right)$ and $G \in \mathrm{E}^{\mathrm{b}}\left(\mathrm{Ik}_{N}\right)$ one has

$$
\begin{aligned}
\mathrm{E} f_{! !} F & \simeq \mathrm{R} \tilde{f}_{! !} \mathrm{L}^{\mathrm{E}}(F) \\
\mathrm{E} f_{*} F & \simeq \mathrm{R} \tilde{f}_{*} \mathrm{~L}^{\mathrm{E}}(F) \simeq \mathrm{R} \tilde{f}_{*} \mathrm{R}^{\mathrm{E}}(F), \\
\mathrm{E} f^{-1} G & \simeq \tilde{f}^{-1} \mathrm{~L}^{\mathrm{E}}(G) \\
\mathrm{E} f^{!} G & \simeq \tilde{f}^{!} \mathrm{L}^{\mathrm{E}}(G) \simeq \tilde{f}^{\mathrm{E}}(G),
\end{aligned}
$$

The above operations satisfy analogous properties as the six operations for sheaves and indsheaves. 
Proposition 6.4.7. Let $f: M \rightarrow N$ be a continuous map of good topological spaces.

(i) The functor $\mathrm{E} f_{\text {!! }}$ is left adjoint to $\mathrm{E} f^{\text {! }}$.

(ii) The functor $\mathrm{E} f^{-1}$ is left adjoint to $\mathrm{E} f_{*}$.

Proposition 6.4.8. Given two continuous maps of good topological spaces $L \stackrel{g}{\rightarrow} M \stackrel{f}{\rightarrow} N$, one has

$$
\mathrm{E}(f \circ g)_{! !} \simeq \mathrm{E} f_{! !} \circ \mathrm{E} g_{! !}, \quad \mathrm{E}(f \circ g)_{*} \simeq \mathrm{E} f_{*} \circ \mathrm{E} g_{*}
$$

and

$$
\mathrm{E}(f \circ g)^{-1} \simeq \mathrm{E} g^{-1} \circ \mathrm{E} f^{-1}, \quad \mathrm{E}(f \circ g)^{!} \simeq \mathrm{E} g^{!} \circ \mathrm{E} f^{!} .
$$

Proposition 6.4.9. Let $f: M \rightarrow N$ be a continuous map of good topological spaces. For $K \in \mathrm{E}^{\mathrm{b}}\left(\mathrm{Ik}_{M}\right)$ and $L, L_{1}, L_{2} \in \mathrm{E}^{\mathrm{b}}\left(\mathrm{Ik}_{N}\right)$, one has isomorphisms

$$
\begin{aligned}
\mathrm{E} f_{! !}\left(\mathrm{E} f^{-1} L \stackrel{+}{\otimes} K\right) & \simeq L \stackrel{+}{\otimes} \mathrm{E} f_{! !} K, \\
\mathrm{E} f^{-1}\left(L_{1} \stackrel{+}{\otimes} L_{2}\right) & \simeq \mathrm{E} f^{-1} L_{1} \stackrel{+}{\otimes} \mathrm{E} f^{-1} L_{2}, \\
\mathscr{I h o m}{ }^{+}\left(L, \mathrm{E} f_{*} K\right) & \simeq \mathrm{E} f_{*} \mathscr{I h o m}{ }^{+}\left(\mathrm{E} f^{-1} L, K\right), \\
\mathscr{I h o m}{ }^{+}\left(\mathrm{E} f_{! !} K, L\right) & \simeq \mathrm{E} f_{*} \mathscr{I h o m}{ }^{+}\left(K, \mathrm{E} f^{!} L\right), \\
\mathrm{E} f^{!} \mathscr{I h o m}^{+}\left(L_{1}, L_{2}\right) & \simeq \mathscr{I} \operatorname{Ihom}^{+}\left(\mathrm{E} f^{-1} L_{1}, \mathrm{E} f^{!} L_{2}\right),
\end{aligned}
$$

and a morphism

$$
\mathrm{E} f^{-1} \mathscr{I h o m}^{+}\left(L_{1}, L_{2}\right) \rightarrow \mathscr{I h o m}^{+}\left(\mathrm{E} f^{-1} L_{1}, \mathrm{E} f^{-1} L_{2}\right) .
$$

Proposition 6.4.10. Consider a Cartesian diagram of good topological spaces

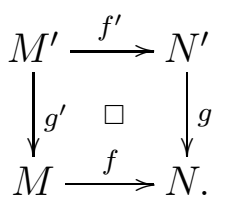

Then there are isomorphisms in the category of functors from $\mathrm{E}^{\mathrm{b}}\left(\mathrm{Ik}_{M}\right)$ to $\mathrm{E}^{\mathrm{b}}\left(\mathrm{Ik}_{N^{\prime}}\right)$ :

$$
\mathrm{E} g^{-1} \mathrm{E} f_{! !} \simeq \mathrm{E} f_{! !}^{\prime} \mathrm{E} g^{\prime-1}, \quad \mathrm{E} g ! \mathrm{E} f_{*} \simeq \mathrm{E} f_{*}^{\prime} \mathrm{E} g^{\prime !} .
$$


Lemma 6.4.11. For $f: M \rightarrow N$ a morphism of good topological spaces, $K \in \mathrm{E}^{\mathrm{b}}\left(\mathrm{Ik}_{M}\right)$ and $L \in \mathrm{E}^{\mathrm{b}}\left(\mathrm{Ik}_{N}\right)$, one has

$$
\begin{aligned}
\mathrm{R} f_{*} \mathscr{H} o m^{\mathrm{E}}\left(K, \mathrm{E} f^{!} L\right) & \simeq \mathscr{H} o m^{\mathrm{E}}\left(\mathrm{E} f_{! !} K, L\right), \\
\mathrm{R} f_{*} \mathscr{H} o m^{\mathrm{E}}\left(\mathrm{E} f^{-1} L, K\right) & \simeq \mathscr{H} o m^{\mathrm{E}}\left(L, \mathrm{E} f_{*} K\right) .
\end{aligned}
$$

Remark 6.4.12. Let $f: M \rightarrow N$ be a morphism of good topological spaces and $L_{1}, L_{2} \in \mathrm{E}^{\mathrm{b}}\left(\mathrm{Ik}_{N}\right)$. Since $\alpha$ and $f^{!}$do not commute in general, the isomorphism $f^{!} \mathscr{H}_{o m}{ }^{\mathrm{E}}\left(L_{1}, L_{2}\right) \simeq \mathscr{H}_{o m}{ }^{\mathrm{E}}\left(\mathrm{E} f^{-1} L_{1}, \mathrm{E} f^{!} L_{2}\right)$ does not hold in general.

\subsection{Stable objects}

The notion of stable object which will be introduced below is related to the notion of torsion object from [Ta 08] (see also [GS12, §5]).

Notation 6.5.1. Consider the indsheaves on $M \times \overline{\mathbb{R}}$

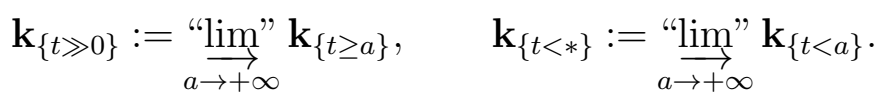

We regard them as objects of $\mathrm{D}^{\mathrm{b}}\left(\mathrm{Ik}_{M \times \mathbb{R}_{\infty}}\right)$.

There is a distinguished triangle in $\mathrm{D}^{\mathrm{b}}\left(\mathrm{Ik}_{M \times \mathbb{R}_{\infty}}\right)$

$$
\mathbf{k}_{M \times \mathbb{R}} \rightarrow \mathbf{k}_{\{t \gg 0\}} \rightarrow \mathbf{k}_{\{t<*\}}[1] \stackrel{+1}{\longrightarrow}
$$

and there are isomorphisms in $\mathrm{D}^{\mathrm{b}}\left(\mathrm{Ik}_{M \times \mathbb{R}_{\infty}}\right)$

$$
\mathbf{k}_{\{t \geq-a\}} \stackrel{+}{\otimes} \mathbf{k}_{\{t \gg 0\}} \stackrel{\sim}{\sim} \mathbf{k}_{\{t \gg 0\}} \stackrel{\sim}{\longrightarrow} \mathbf{k}_{\{t \geq a\}} \stackrel{+}{\otimes} \mathbf{k}_{\{t \gg 0\}} \quad\left(a \in \mathbb{R}_{\geq 0}\right) .
$$

Notation 6.5.2. Denote by $\mathbf{k}_{M}^{\mathrm{E}}$ the object of $\mathrm{E}^{\mathrm{b}}\left(\mathrm{Ik}_{M}\right)$ associated with $\mathbf{k}_{\{t \gg 0\}} \in \mathrm{D}^{\mathrm{b}}\left(\mathrm{Ik}_{M \times \mathbb{R}_{\infty}}\right)$. More generally, for $F \in \mathrm{D}^{\mathrm{b}}\left(\mathbf{k}_{M}\right)$, set

$$
F^{\mathrm{E}}:=\mathbf{k}_{M}^{\mathrm{E}} \otimes \pi^{-1} F \in \mathrm{E}^{\mathrm{b}}\left(\mathrm{Ik}_{M}\right) .
$$

Note that

$$
\mathrm{L}^{\mathrm{E}}\left(\mathbf{k}_{M}^{\mathrm{E}}\right) \simeq \mathbf{k}_{\{t \gg 0\}}, \quad \mathrm{R}^{\mathrm{E}}\left(\mathbf{k}_{M}^{\mathrm{E}}\right) \simeq \mathbf{k}_{\{t<*\}}[1] .
$$

Proposition 6.5.3. Let $K \in \mathrm{E}_{+}^{\mathrm{b}}\left(\mathrm{Ik}_{M}\right)$ (equivalently, $K \in \mathrm{E}^{\mathrm{b}}\left(\mathrm{Ik}_{M}\right)$ and $\left.K \simeq \mathbf{k}_{\{t \geq 0\}} \stackrel{+}{\otimes} K\right)$. Then the following conditions are equivalent. 
(a) $\mathbf{k}_{\{t \geq 0\}} \stackrel{+}{\otimes} K \stackrel{\sim}{\longrightarrow} \mathbf{k}_{\{t \geq a\}} \stackrel{+}{\otimes} K$ for any $a \geq 0$,

(b) $\mathscr{I h o m}^{+}\left(\mathbf{k}_{\{t \geq a\}}, K\right) \stackrel{\sim}{\longrightarrow} \mathscr{I h o m}^{+}\left(\mathbf{k}_{\{t \geq 0\}}, K\right)$ for any $a \geq 0$,

(c) $\mathbf{k}_{\{t \geq 0\}} \stackrel{+}{\otimes} K \stackrel{\sim}{\longrightarrow} \mathbf{k}_{M}^{\mathrm{E}} \stackrel{+}{\otimes} K$,

(d) $\mathscr{I h o m}^{+}\left(\mathbf{k}_{M}^{\mathrm{E}}, K\right) \stackrel{\sim}{\longrightarrow} \mathscr{I h o m}^{+}\left(\mathbf{k}_{\{t \geq 0\}}, K\right)$,

(e) $K \simeq \mathbf{k}_{M}^{\mathrm{E}} \stackrel{+}{\otimes} L$ for some $L \in \mathrm{E}^{\mathrm{b}}\left(\mathrm{Ik}_{M}\right)$,

(f) $K \simeq \mathscr{I h o m}^{+}\left(\mathbf{k}_{M}^{\mathrm{E}}, L\right)$ for some $L \in \mathrm{E}^{\mathrm{b}}\left(\mathrm{Ik}_{M}\right)$.

Definition 6.5.4. A stable object is an object of $\mathrm{E}_{+}^{\mathrm{b}}\left(\mathrm{Ik}_{M}\right)$ that satisfies the equivalent conditions in Proposition 6.5.3.

Lemma 6.5.5. For $F \in \mathrm{D}^{\mathrm{b}}\left(\mathbf{k}_{M \times \mathbb{R}_{\infty}}\right)$ and $K \in \mathrm{E}^{\mathrm{b}}\left(\mathrm{Ik}_{M}\right)$, there is an isomorphism in $\mathrm{E}^{\mathrm{b}}\left(\mathrm{Ik}_{M}\right)$

$$
\mathbf{k}_{M}^{\mathrm{E}} \stackrel{+}{\otimes} \mathscr{I h o m}^{+}(F, K) \stackrel{\sim}{\longrightarrow} \mathscr{I h o m}^{+}\left(F, \mathbf{k}_{M}^{\mathrm{E}} \stackrel{+}{\otimes} K\right)
$$

Corollary 6.5.6. For $K \in \mathrm{E}^{\mathrm{b}}\left(\mathrm{I}_{M}\right)$ and $F \in \mathrm{D}^{\mathrm{b}}\left(\mathbf{k}_{M}\right)$, we have

$$
\mathbf{k}_{M}^{\mathrm{E}} \stackrel{+}{\otimes} \mathrm{R} \mathscr{I} h o m\left(\pi^{-1} F, K\right) \simeq \mathrm{R} \mathscr{I h o m}\left(\pi^{-1} F, \mathbf{k}_{M}^{\mathrm{E}} \stackrel{+}{\otimes} K\right) .
$$

Proposition 6.5.7. Let $f: M \rightarrow N$ be a continuous map of good topological spaces. Then the functors $\mathrm{E} f_{! !}, \mathrm{E} f^{-1}$ and $\mathrm{E} f^{!}$send stable objects to stable objects. More precisely, we have:

(i) For $K \in \mathrm{E}^{\mathrm{b}}\left(\mathrm{Ik}_{M}\right)$ one has

$$
\mathrm{E} f_{! !}\left(\mathbf{k}_{M}^{\mathrm{E}} \stackrel{+}{\otimes} K\right) \simeq \mathbf{k}_{N}^{\mathrm{E}} \stackrel{+}{\otimes} \mathrm{E} f_{! !} K .
$$

(ii) For $L \in \mathrm{E}^{\mathrm{b}}\left(\mathrm{Ik}_{N}\right)$ one has

$$
\begin{aligned}
\mathrm{E} f^{-1}\left(\mathbf{k}_{N}^{\mathrm{E}} \stackrel{+}{\otimes} L\right) & \simeq \mathbf{k}_{M}^{\mathrm{E}} \stackrel{+}{\otimes} \mathrm{E} f^{-1} L, \\
\mathrm{E} f^{!}\left(\mathbf{k}_{N}^{\mathrm{E}} \stackrel{+}{\otimes} L\right) & \simeq \mathbf{k}_{M}^{\mathrm{E}} \stackrel{+}{\otimes} \mathrm{E} f^{!} L .
\end{aligned}
$$


Definition 6.5.8. One defines the functors

$$
\begin{aligned}
e_{M}, \epsilon_{M}: \mathrm{D}^{\mathrm{b}}\left(\mathrm{Ik}_{M}\right) & \rightarrow \mathrm{E}^{\mathrm{b}}\left(\mathrm{I}_{M}\right), \\
e_{M}(F) & =\mathbf{k}_{M}^{\mathrm{E}} \otimes \pi^{-1} F, \quad \epsilon_{M}(F)=\mathbf{k}_{\{t \geq 0\}} \otimes \pi^{-1} F .
\end{aligned}
$$

Note that

$$
e_{M}(F) \simeq \mathbf{k}_{M}^{\mathrm{E}} \stackrel{+}{\otimes} \epsilon_{M}(F) .
$$

Proposition 6.5.9. The functors $e_{M}$ and $\epsilon_{M}$ are fully faithful.

Definition 6.5.10. We define the duality functor

$$
\mathrm{D}_{M}^{\mathrm{E}}: \mathrm{E}^{\mathrm{b}}\left(\mathrm{Ik}_{M}\right) \rightarrow \mathrm{E}^{\mathrm{b}}\left(\mathrm{Ik}_{M}\right)^{\mathrm{op}}, \quad K \mapsto \mathscr{I} h o m^{+}\left(K, \omega_{M}^{\mathrm{E}}\right),
$$

where $\omega_{M}^{\mathrm{E}}:=\mathbf{k}_{M}^{\mathrm{E}} \otimes \pi^{-1} \omega_{M}$.

The functor $\mathrm{D}_{M}^{\mathrm{E}}$ is related to the usual duality functor for sheaves by the formula:

$$
\mathrm{D}_{M}^{\mathrm{E}}\left(\mathbf{k}_{M}^{\mathrm{E}} \stackrel{+}{\otimes} F\right) \simeq \mathbf{k}_{M}^{\mathrm{E}} \stackrel{+}{\otimes} a^{-1} \mathrm{D}_{M \times \mathbb{R}} F \quad \text { in } \mathrm{E}^{\mathrm{b}}\left(\mathrm{Ik}_{M}\right),
$$

where $F \in \mathrm{D}^{\mathrm{b}}\left(\mathbf{k}_{M \times \mathbb{R}_{\infty}}\right)$ and $a$ is the involution of $M \times \mathbb{R}$ given by $(x, t) \mapsto$ $(x,-t)$.

\subsection{Constructible enhanced indsheaves}

In this subsection, we assume that $M$ is a subanalytic space. Recall the natural morphism of bordered spaces

$$
j_{M}: M \times \mathbb{R}_{\infty} \rightarrow M \times \overline{\mathbb{R}}
$$

and the category $\mathrm{D}^{\mathrm{b}}\left(\mathbf{k}_{M \times \mathbb{R}_{\infty}}\right)$ in Notation 6.2.2.

Definition 6.6.1. One denotes by $\mathrm{D}_{\mathbb{R}-\mathrm{c}}^{\mathrm{b}}\left(\mathbf{k}_{M \times \mathbb{R}_{\infty}}\right)$ the full subcategory of $\mathrm{D}^{\mathrm{b}}\left(\mathbf{k}_{M \times \mathbb{R}_{\infty}}\right)$ whose objects $F$ are such that $\mathrm{R} j_{M !} F$ is $\mathbb{R}$-constructible.

We regard $\mathrm{D}_{\mathbb{R}-\mathrm{c}}^{\mathrm{b}}\left(\mathbf{k}_{M \times \mathbb{R}_{\infty}}\right)$ as a full subcategory of $\mathrm{D}^{\mathrm{b}}\left(\mathrm{Ik}_{M \times \mathbb{R}_{\infty}}\right)$.

Definition 6.6.2. One says that an object $K \in \mathrm{E}^{\mathrm{b}}\left(\mathrm{Ik}_{M}\right)$ is $\mathbb{R}$-constructible if for any relatively compact subanalytic open subset $U \subset M$ there exists an isomorphism

$$
\pi^{-1} \mathbf{k}_{U} \otimes K \simeq \mathbf{k}_{M}^{\mathrm{E}} \stackrel{+}{\otimes} F \text { for some } F \in \mathrm{D}_{\mathbb{R}-\mathrm{c}}^{\mathrm{b}}\left(\mathbf{k}_{M \times \mathbb{R}_{\infty}}\right) .
$$

One denotes by $\mathrm{E}_{\mathbb{R} \text {-c }}^{\mathrm{b}}\left(\mathrm{Ik}_{M}\right)$ the full subcategory of $\mathrm{E}^{\mathrm{b}}\left(\mathrm{Ik}_{M}\right)$ consisting of $\mathbb{R}$ constructible objects. 
Clearly, $\mathbb{R}$-constructible objects of $\mathrm{E}^{\mathrm{b}}\left(\mathrm{Ik}_{M}\right)$ are stable. One proves that:

\section{Theorem 6.6.3.}

(i) The category $\mathrm{E}_{\mathbb{R}-c}^{\mathrm{b}}\left(\mathrm{Ik}_{M}\right)$ is triangulated.

(ii) The property for $K \in \mathrm{E}^{\mathrm{b}}\left(\mathrm{Ik}_{M}\right)$ of being $\mathbb{R}$-constructible is a local property over $M$.

(iii) The functors $\stackrel{+}{\otimes}$ and $\mathscr{I}$ hom ${ }^{+}$when restricted to $\mathbb{R}$-constructible objects give $\mathbb{R}$-constructible objects.

(iv) For $K \in \mathrm{E}_{\mathbb{R}-c}^{\mathrm{b}}\left(\mathrm{Ik}_{M}\right), \mathrm{D}_{M}^{\mathrm{E}} K \in \mathrm{E}_{\mathbb{R}-c}^{\mathrm{b}}\left(\mathrm{Ik}_{M}\right)$ and $\mathrm{D}_{M}^{\mathrm{E}} \circ \mathrm{D}_{M}^{\mathrm{E}} K \simeq K$.

(v) For $K_{1}, K_{2} \in \mathrm{E}_{\mathbb{R}-c}^{\mathrm{b}}\left(\mathrm{Ik}_{M}\right), \mathrm{D}_{M}^{\mathrm{E}}\left(\mathscr{I} h o m^{+}\left(K_{1}, K_{2}\right)\right) \simeq K_{1} \stackrel{+}{\otimes} \mathrm{D}_{M}^{\mathrm{E}} K_{2}$.

(vi) For $K_{1}, K_{2} \in \mathrm{E}_{\mathbb{R}-c}^{\mathrm{b}}\left(\mathrm{Ik}_{M}\right), \mathscr{I} h o m^{\mathrm{E}}\left(K_{1}, K_{2}\right) \simeq \mathscr{I} h o m^{\mathrm{E}}\left(\mathrm{D}_{M}^{\mathrm{E}} K_{2}, \mathrm{D}_{M}^{\mathrm{E}} K_{1}\right)$ and $\mathscr{H}_{o m}^{\mathrm{E}}\left(K_{1}, K_{2}\right) \simeq \mathscr{H}_{o m}^{\mathrm{E}}\left(\mathrm{D}_{M}^{\mathrm{E}} K_{2}, \mathrm{D}_{M}^{\mathrm{E}} K_{1}\right)$.

(vii) Let $f: M \rightarrow N$ be a morphism of subanalytic spaces.

(a) If $G \in \mathrm{E}_{\mathbb{R}-c}^{\mathrm{b}}\left(\mathrm{Ik}_{N}\right)$, then $\mathrm{E} f^{-1} G$ and $\mathrm{E} f ! G$ belong to $\mathrm{E}_{\mathbb{R}-c}^{\mathrm{b}}\left(\mathrm{Ik}_{M}\right)$.

(b) For $K \in \mathrm{E}_{\mathbb{R}-c}^{\mathrm{b}}\left(\mathrm{Ik}_{M}\right)$, we have $\mathrm{E} f_{! !} K \in \mathrm{E}_{\mathbb{R}-c}^{\mathrm{b}}\left(\mathrm{Ik}_{N}\right)$ if $\operatorname{Supp}^{\mathrm{E}}(K):=$ $\bar{\pi}_{M}\left(\operatorname{Supp}\left(\mathrm{R} j_{M *} \mathrm{R}^{\mathrm{E}} K\right)\right)$ is proper over $N$. Here $j_{M}: M \times \mathbb{R}_{\infty} \rightarrow$ $M \times \overline{\mathbb{R}}$ is the inclusion and $\bar{\pi}_{M}: M \times \overline{\mathbb{R}} \rightarrow M$ is the projection.

Another link between classical $\mathbb{R}$-constructible sheaves and enhanced $\mathbb{R}$ constructible indsheaves is given by the following result, which is new:

Theorem 6.6.4. For $F, G \in \mathrm{E}_{\mathbb{R}-c}^{\mathrm{b}}\left(\mathrm{Ik}_{M}\right)$, the object $\mathscr{H}_{o m}^{\mathrm{E}}(F, G)$ belongs to $\mathrm{D}_{\mathbb{R}-c}^{\mathrm{b}}\left(\mathbf{k}_{M}\right)$.

Proof. Since

$$
\mathscr{H} o m^{\mathrm{E}}(F, G) \simeq{\mathscr{H} o m^{\mathrm{E}}}\left(\mathbf{k}_{\{t \geq 0\}}, \mathscr{I h o m}^{+}(F, G)\right),
$$

it is enough to show that

$$
\mathscr{H}_{o m}^{\mathrm{E}}\left(\mathbf{k}_{\{t \geq 0\}}, F\right) \in \mathrm{D}_{\mathbb{R}-\mathrm{c}}^{\mathrm{b}}\left(\mathbf{k}_{M}\right)
$$

for any $F \in \mathrm{E}_{\mathbb{R}-\mathrm{c}}^{\mathrm{b}}\left(\mathrm{Ik}_{M}\right)$. Now it follows from Corollary 6.6.6 below which is a consequence of the following proposition.

Q.E.D. 
Proposition 6.6.5. For $K \in \mathrm{D}^{\mathrm{b}}\left(\mathrm{Ik}_{M \times \mathbb{R}_{\infty}}\right)$, we have

$$
\mathrm{R} \pi_{*} \mathrm{R} \mathscr{I h o m}\left(\mathbf{k}_{\{t \geq 0\}}, \mathbf{k}_{\{t \gg 0\}} \stackrel{+}{\otimes} K\right) \simeq \mathrm{R} \pi_{*}\left(\mathbf{k}_{\{t>*\}} \otimes K\right) .
$$

Here $\mathbf{k}_{\{t>*\}}:=\underset{a \rightarrow-\infty}{\stackrel{\lim }{\rightarrow}} \mathbf{k}_{\{t>a\}} \in \mathrm{I}\left(\mathbf{k}_{M \times \mathbb{R}_{\infty}}\right)$.

Proof. We shall first show that

$$
\begin{aligned}
H^{n} \mathrm{R} \pi_{*} \mathrm{R} \mathscr{I h o m}\left(\mathbf{k}_{\{t \geq 0\}}, \mathbf{k}_{\{t \gg 0\}} \stackrel{+}{\otimes} K\right) & \\
& \simeq \underset{a \rightarrow-\infty}{\stackrel{\lim "}{\rightarrow}} H^{n} \mathrm{R} \pi_{*} \mathrm{R} \mathscr{I h o m}\left(\mathbf{k}_{\{t \geq a\}}, K\right) \\
& \simeq \underset{a}{\stackrel{\lim }{\rightarrow} "-\infty} H^{n} \mathrm{R} \pi_{*}\left(\mathbf{k}_{\{t>a\}} \otimes K\right) .
\end{aligned}
$$

The first isomorphism in (6.6.2) follows from

$$
\begin{aligned}
& H^{n} \mathrm{R} \pi_{*} \mathrm{R} \mathscr{I h o m}\left(\mathbf{k}_{\{t \geq 0\}}, \mathbf{k}_{\{t \gg 0\}} \stackrel{+}{\otimes} K\right) \\
& \simeq \underset{a \rightarrow+\infty}{\stackrel{\lim }{\rightarrow}} H^{n} \mathrm{R} \pi_{*} \mathrm{R} \mathscr{I h o m}\left(\mathbf{k}_{\{t \geq 0\}}, \mathbf{k}_{\{t \geq a\}} \stackrel{+}{\otimes} K\right) \\
& \underset{(\mathrm{a})}{\sim} \underset{a \rightarrow+\infty}{\stackrel{\lim }{\rightarrow}} H^{n} \mathrm{R} \pi_{*} \mathrm{R} \mathscr{I h o m}\left(\mathbf{k}_{\{t \geq 0\}}, \mathscr{I h o m}{ }^{+}\left(\mathbf{k}_{\{t \geq-a\}}, K\right)\right) \\
& \simeq \underset{a \rightarrow+\infty}{\stackrel{\lim }{\longrightarrow}} H^{n} \mathrm{R} \pi_{*} \mathrm{R} \mathscr{I h o m}\left(\mathbf{k}_{\{t \geq-a\}}, K\right) \text {. }
\end{aligned}
$$

Here, isomorphism (a) follows from Proposition 6.2 .12 and $R \pi_{! !}\left(\mathbf{k}_{\{t \geq 0\}}\right) \simeq 0$.

Let us next show the second isomorphism in (6.6.2). There is a sequence of morphisms

$$
\begin{aligned}
& \underset{a \rightarrow-\infty}{\stackrel{\lim }{\rightarrow}} H^{n} \mathrm{R} \pi_{*}\left(\mathbf{k}_{\{t>a\}} \otimes K\right) \stackrel{f_{1}}{\longrightarrow} \underset{a \rightarrow-\infty}{\stackrel{\lim }{\rightarrow}} H^{n} \mathrm{R} \pi_{*} \mathrm{R} \mathscr{I} h o m\left(\mathbf{k}_{\{t \geq a\}}, K\right) \\
& \stackrel{f_{2}}{\longrightarrow} \underset{a \rightarrow-\infty}{\stackrel{\lim }{\longrightarrow}} H^{n} \mathrm{R} \pi_{*}\left(\mathbf{k}_{\{t>a-1\}} \otimes K\right) \\
& \stackrel{f_{3}}{\longrightarrow} \underset{a \rightarrow-\infty}{\stackrel{\lim }{\longrightarrow}} H^{n} \mathrm{R} \pi_{*} \mathrm{R} \mathscr{I} h o m\left(\mathbf{k}_{\{t \geq a-1\}}, K\right) .
\end{aligned}
$$

Since $f_{2} f_{1}$ and $f_{3} f_{2}$ are isomorphisms, $f_{1}$ is an isomorphism.

Thus we have proved (6.6.2). 
Then (6.6.2) implies that

$$
H^{n} \mathrm{R} \pi_{*} \mathrm{R} \mathscr{I} h o m\left(\mathbf{k}_{\{t \geq 0\}}, \mathbf{k}_{\{t \gg 0\}} \stackrel{+}{\otimes} j_{M}^{-1} L\right) \simeq H^{n} \mathrm{R} \pi_{*}\left(\mathbf{k}_{\{t>*\}} \otimes j_{M}^{-1} L\right) \simeq 0
$$

for any $n \in \mathbb{Z} \backslash\{0\}$ and any quasi-injective $L \in \mathrm{I}\left(\mathbf{k}_{M \times \overline{\mathbb{R}}}\right)$. Here $j_{M}: M \times$ $\mathbb{R}_{\infty} \rightarrow M \times \overline{\mathbb{R}}$ is the natural morphism bordered spaces. Therefore, the functor $\mathrm{R} \pi_{*} \mathrm{R} \mathscr{I} h o m\left(\mathbf{k}_{\{t \geq 0\}}, \mathbf{k}_{\{t \gg 0\}} \stackrel{+}{\otimes} j_{M}^{-1} \bullet\right)$ is isomorphic to the right derived functor of $H^{0} \mathrm{R} \pi_{*} \mathrm{R} \mathscr{I h o m}\left(\mathbf{k}_{\{t \geq 0\}}, \mathbf{k}_{\{t \gg 0\}} \stackrel{+}{\otimes} j_{M}^{-1} \bullet\right)$. Similarly, $\mathrm{R} \pi_{*}\left(\mathbf{k}_{\{t>*\}} \otimes\right.$ $\left.j_{M}^{-1} \bullet\right)$ is isomorphic to the right derived functor of $H^{0} \mathrm{R} \pi_{*}\left(\mathbf{k}_{\{t>*\}} \otimes j_{M}^{-1} \bullet\right)$. Since $H^{0} \mathrm{R} \pi_{*} \mathrm{R} \mathscr{I h o m}\left(\mathbf{k}_{\{t \geq 0\}}, \mathbf{k}_{\{t \gg 0\}} \stackrel{+}{\otimes} j_{M}^{-1} \bullet\right)$ and $H^{0} \mathrm{R} \pi_{*}\left(\mathbf{k}_{\{t>*\}} \otimes j_{M}^{-1} \bullet\right)$ are isomorphic by (6.6.2), we obtain the desired result. Q.E.D.

Corollary 6.6.6. For any $F \in \mathrm{D}^{\mathrm{b}}\left(\mathbf{k}_{M \times \mathbb{R}_{\infty}}\right)$, we have an isomorphism in $\mathrm{D}^{\mathrm{b}}\left(\mathbf{k}_{M}\right)$ :

$$
\mathscr{H}_{o m}{ }^{\mathrm{E}}\left(\mathbf{k}_{\{t \geq 0\}}, \mathbf{k}_{M}^{\mathrm{E}} \stackrel{+}{\otimes} F\right) \simeq \mathrm{R} \bar{\pi}_{*}\left(\mathbf{k}_{M \times(\overline{\mathbb{R}} \backslash\{-\infty\})} \otimes \mathrm{R} j_{M *} F\right),
$$

where $\bar{\pi}: M \times \overline{\mathbb{R}} \rightarrow M$ is the projection and $j_{M}: M \times \mathbb{R} \rightarrow M \times \overline{\mathbb{R}}$ is the inclusion.

Proof. We have

$$
\begin{aligned}
\mathrm{R} \pi_{*}\left(\mathbf{k}_{\{t>*\}} \otimes F\right) & \simeq \mathrm{R} \bar{\pi}_{*} \mathrm{R} j_{M_{*}}\left(\mathbf{k}_{\{t>*\}} \otimes F\right) \\
& \simeq \mathrm{R} \bar{\pi}_{*}\left(\mathbf{k}_{\{+\infty \geq t>*\}} \otimes \mathrm{R} j_{M_{*}} F\right),
\end{aligned}
$$

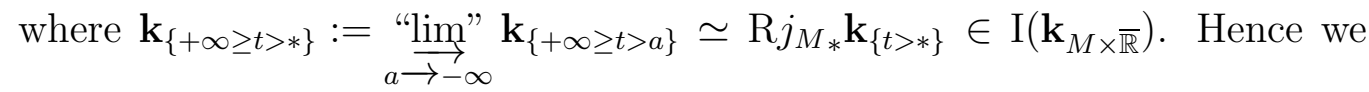
have

$$
\begin{aligned}
\mathscr{H}_{o m}{ }^{\mathrm{E}}\left(\mathbf{k}_{\{t \geq 0\}}, \mathbf{k}_{M}^{\mathrm{E}} \stackrel{+}{\otimes} F\right) & \simeq \alpha_{M} \mathscr{I} h m^{\mathrm{E}}\left(\mathbf{k}_{\{t \geq 0\}}, \mathbf{k}_{M}^{\mathrm{E}} \stackrel{+}{\otimes} F\right) \\
& \simeq \alpha_{M} \mathrm{R} \bar{\pi}_{*}\left(\mathbf{k}_{\{+\infty \geq t>*\}} \otimes \mathrm{R} j_{M *} F\right) \\
& \simeq \mathrm{R} \bar{\pi}_{*} \alpha_{M \times \overline{\mathbb{R}}}\left(\mathbf{k}_{\{+\infty \geq t>*\}} \otimes \mathrm{R} j_{M *} F\right) \\
& \simeq \mathrm{R} \bar{\pi}_{*}\left(\mathbf{k}_{M \times(\overline{\mathbb{R}} \backslash\{-\infty\})} \otimes \mathrm{R} j_{M_{*}} F\right) .
\end{aligned}
$$

Q.E.D. 


\subsection{Enhanced indsheaves with ring action}

Let $\mathcal{A}$ be a sheaf of $\mathbf{k}$-algebras on $M$. For $\dagger=, \mathrm{b},+,-$, we define

$$
\mathrm{D}^{\dagger}\left(\mathrm{I}\left(\pi^{-1} \mathcal{A}\right)\right):=\mathrm{D}^{\dagger}\left(\mathrm{I}\left(\bar{\pi}^{-1} \mathcal{A}\right)\right) / \mathrm{D}^{\dagger}\left(\mathrm{I}\left(\left.\left(\bar{\pi}^{-1} \mathcal{A}\right)\right|_{M \times(\overline{\mathbb{R}} \backslash \mathbb{R})}\right)\right),
$$

where $\bar{\pi}: M \times \overline{\mathbb{R}} \rightarrow M$ is the projection. Then we set

$\mathrm{E}^{\dagger}(\mathrm{I} \mathcal{A})=\mathrm{D}^{\dagger}\left(\mathrm{I}\left(\pi^{-1} \mathcal{A}\right)\right) /\left\{K \in \mathrm{D}^{\dagger}\left(\mathrm{I}\left(\pi^{-1} \mathcal{A}\right)\right) ; K \simeq \pi^{-1} L\right.$ for some $\left.L \in \mathrm{D}^{\dagger}(\mathrm{I} \mathcal{A})\right\}$.

We call objects of $\mathrm{E}^{\mathrm{b}}(\mathrm{I} \mathcal{A})$ enhanced indsheaves with $\mathcal{A}$-action.

We can define also the functors

$$
\begin{aligned}
& \stackrel{+}{\otimes}_{\beta \mathcal{A}}: \mathrm{E}^{\mathrm{b}}\left(\mathrm{I} \mathcal{A}^{\mathrm{op}}\right) \times \mathrm{E}^{\mathrm{b}}(\mathrm{I} \mathcal{A}) \rightarrow \mathrm{E}^{-}\left(\mathrm{Ik}_{M}\right), \\
& \mathscr{I h o m}_{\beta \mathcal{A}}^{+}: \mathrm{E}^{\mathrm{b}}(\mathrm{I} \mathcal{A})^{\mathrm{op}} \times \mathrm{E}^{\mathrm{b}}(\mathrm{I} \mathcal{A}) \rightarrow \mathrm{E}^{+}\left(\mathrm{Ik}_{M}\right) \text {, }
\end{aligned}
$$

which satisfy similar properties to $\stackrel{+}{\otimes}$ and $\mathscr{I} \mathrm{hom}^{+}$.

Similarly we can define

$$
\begin{array}{r}
\stackrel{\mathrm{L}}{\otimes}_{\mathcal{A}}: \mathrm{E}^{\mathrm{b}}\left(\mathrm{I} \mathcal{A}^{\mathrm{op}}\right) \times \mathrm{D}^{\mathrm{b}}(\mathcal{A}) \rightarrow \mathrm{E}^{-}\left(\mathrm{Ik}_{M}\right), \\
\mathrm{R} \mathscr{H} o m_{\mathcal{A}}: \mathrm{D}^{\mathrm{b}}(\mathcal{A})^{\mathrm{op}} \times \mathrm{E}^{\mathrm{b}}(\mathrm{I} \mathcal{A}) \rightarrow \mathrm{E}^{-}\left(\mathrm{Ik}_{M}\right) .
\end{array}
$$

If $X$ is a complex manifold and $\mathcal{A}=\mathscr{D}_{X}$, we can define

$$
\stackrel{\mathrm{D}}{\otimes}: \mathrm{E}^{\mathrm{b}}\left(\mathrm{I}_{\mathscr{D}_{X}^{\mathrm{p}}}^{\mathrm{p}}\right) \times \mathrm{D}^{\mathrm{b}}\left(\mathscr{D}_{X}\right) \rightarrow \mathrm{E}^{-}\left(\mathrm{I} \mathscr{D}_{X}\right) .
$$

\section{Holonomic D-modules}

\subsection{Exponential D-modules}

Let $X$ be a complex analytic manifold, $Y \subset X$ a complex analytic hypersurface and set $U=X \backslash Y$. For $\varphi \in \mathscr{O}_{X}(* Y)$, one sets

$$
\begin{aligned}
\mathscr{D}_{X} \mathrm{e}^{\varphi} & =\mathscr{D}_{X} /\left\{P ; P \mathrm{e}^{\varphi}=0 \text { on } U\right\}, \\
\mathscr{E}_{U \mid X}^{\varphi} & =\mathscr{D}_{X} \mathrm{e}^{\varphi}(* Y) .
\end{aligned}
$$

Hence $\mathscr{D}_{X} \mathrm{e}^{\varphi}$ is a $\mathscr{D}_{X}$-submodule of $\mathscr{E}_{U \mid X}^{\varphi}$, and $\mathscr{D}_{X} \mathrm{e}^{\varphi}$ as well as $\mathscr{E}_{U \mid X}^{\varphi}$ are holonomic $\mathscr{D}_{X}$-modules. Note that $\mathscr{E}_{U \mid X}^{\varphi}$ is isomorphic to $\mathscr{O}_{X}(* Y)$ as an 
$\mathscr{O}_{X}$-module, and the connection $\mathscr{O}_{X}(* Y) \rightarrow \Omega_{X}^{1} \otimes_{\mathscr{O}_{X}} \mathscr{O}_{X}(* Y)$ is given by $u \mapsto d u+u d \varphi$.

For $c \in \mathbb{R}$, set for short

$$
\{\operatorname{Re} \varphi<c\}:=\{x \in U ; \operatorname{Re} \varphi(x)<c\} \subset X .
$$

Notation 7.1.1. One sets

$$
\begin{aligned}
\mathbb{C}_{\{\operatorname{Re} \varphi<*\}} & :=\underset{c \rightarrow+\infty}{\text { "lim" }} \mathbb{C}_{\{\operatorname{Re} \varphi<c\}} \in \mathrm{I}_{X}, \\
E_{U \mid X}^{\varphi} & :=\operatorname{R} \mathscr{I h o m}\left(\mathbb{C}_{U}, \mathbb{C}_{\{\operatorname{Re} \varphi<*\}}\right) \in \mathrm{D}^{\mathrm{b}}\left(\mathrm{I} \mathbb{C}_{X}\right) .
\end{aligned}
$$

For example, denoting by $z \in \mathbb{C} \subset \mathrm{P}$ the affine coordinate of the complex projective line, one has

$$
H^{j} E_{\mathbb{C} \mid \mathrm{P}}^{z} \simeq \begin{cases}\mathbb{C}_{\{\operatorname{Re} z<*\}} & \text { for } j=0 \\ \mathbb{C}_{\{\infty\}} & \text { for } j=1 \\ 0 & \text { otherwise }\end{cases}
$$

The next result (see [DK 13, Prop. 6.2.2]) generalizes [KS 03, Proposition 7.3] in which the case $X=\mathbb{C}$ and $\varphi(z)=1 / z$ was treated (and recalled in $\S 4.5)$.

Proposition 7.1.2. Let $Y \subset X$ be a closed complex analytic hypersurface and set $U=X \backslash Y$. For $\varphi \in \mathscr{O}_{X}(* Y)$, there is an isomorphism in $\mathrm{D}^{\mathrm{b}}\left(\mathrm{I} \mathbb{C}_{X}\right)$

$$
\mathcal{D} \mathcal{R}_{X}^{\mathrm{t}}\left(\mathscr{E}_{U \mid X}^{-\varphi}\right) \simeq E_{U \mid X}^{\varphi}\left[d_{X}\right] .
$$

\subsection{Enhanced tempered holomorphic functions}

Consider first a real analytic manifold $M$ and the natural morphism of bordered spaces

$$
j: M \times \mathbb{R}_{\infty} \rightarrow M \times \mathbb{P}^{1}(\mathbb{R}) .
$$

Let $t$ be a coordinate of $\mathbb{R}_{\infty}$.

Definition 7.2.1. One sets $\mathcal{D} b_{M \times \mathbb{R}_{\infty}}^{\mathrm{t}}:=j^{!} \mathcal{D} b_{M \times \mathbb{P}^{1}(\mathbb{R})}^{\mathrm{t}}$ and one denotes by $\mathcal{D} b_{M}^{\top} \in \mathrm{D}^{\mathrm{b}}\left(\mathrm{IC}_{M \times \mathbb{R}_{\infty}}\right)$ the complex, concentrated in degree -1 and 0 :

$$
\mathcal{D} b_{M}^{\top}:=\mathcal{D} b_{M \times \mathbb{R}_{\infty}}^{\mathrm{t}} \stackrel{\partial_{t}-1}{\longrightarrow} \mathcal{D} b_{M \times \mathbb{R}_{\infty}}^{\mathrm{t}} .
$$


Note that $H^{k}\left(\mathcal{D} b_{M}^{\top}\right)=0$ for $k \neq-1$.

Proposition 7.2.2. There are isomorphisms in $\mathrm{D}^{\mathrm{b}}\left(\mathrm{IC}_{M \times \mathbb{R}_{\infty}}\right)$

$$
\begin{aligned}
\mathcal{D} b_{M}^{\top} & \stackrel{\sim}{\sim} \operatorname{Ihom}^{+}\left(\mathbb{C}_{\{t \geq 0\}}, \mathcal{D} b_{M}^{\top}\right) \\
& \sim \mathscr{I h o m}^{+}\left(\mathbb{C}_{\{t \geq a\}}, \mathcal{D} b_{M}^{\top}\right) \quad \text { for any } a \geq 0 .
\end{aligned}
$$

Moreover, denoting by $\iota: M \times \mathbb{R} \rightarrow M \times \mathbb{R}_{\infty}$ the natural morphism, one has the isomorphism $\iota^{-1} \mathcal{D} b_{M}^{\top} \simeq \iota^{-1} \pi^{-1} \mathcal{D} b_{M}^{\mathrm{t}}[1]$ and therefore:

$$
\mathscr{I} h o m^{\mathrm{E}}\left(\mathbb{C}_{\{t=0\}}, \mathcal{D} b_{M}^{\mathrm{T}}\right) \simeq \mathcal{D} b_{M}^{\mathrm{t}}
$$

Now let $X$ be again a complex manifold.

Definition 7.2.3. One sets

$$
\begin{aligned}
\mathscr{O}_{X}^{\mathrm{E}} & =\mathrm{R} \mathscr{H} O m_{\pi^{-1} \mathscr{D}_{X^{c}}}\left(\pi^{-1} \mathscr{O}_{X^{c}}, \mathcal{D} b_{X_{\mathbb{R}}}^{\mathrm{T}}\right), \\
\Omega_{X}^{\mathrm{E}} & =\pi^{-1} \Omega_{X} \otimes_{\pi^{-1} \mathscr{O}_{X}} \mathscr{O}_{X}^{\mathrm{E}} .
\end{aligned}
$$

We regard them as objects of $\mathrm{E}^{\mathrm{b}}\left(\mathrm{I} \mathscr{D}_{X}\right)$ and $\mathrm{E}^{\mathrm{b}}\left(\mathrm{I} \mathscr{D}_{X}^{\mathrm{op}}\right)$, respectively. One calls $\mathscr{O}_{X}^{\mathrm{E}}$ the enhanced indsheaf of tempered holomorphic functions.

Remark 7.2.4. When $X=\mathrm{pt}$, we have $\mathcal{D} b_{X}^{\top} \simeq \mathbb{C}_{\{t<*\}}[1]$ (see Notation 6.5.1) as an object of $\mathrm{D}^{\mathrm{b}}\left(\mathrm{I}_{\mathbb{R}_{\infty}}\right)$ and $\mathscr{O}_{X}^{\mathrm{E}} \simeq \mathbb{C}_{X}^{\mathrm{E}}$ as an object of $\mathrm{E}^{\mathrm{b}}\left(\mathrm{I} \mathbb{C}_{X}\right)$.

Applying Proposition 7.2.2, we get

Proposition 7.2.5. There are isomorphisms in $\mathrm{E}^{\mathrm{b}}\left(\mathrm{I} \mathscr{D}_{X}\right)$

$$
\begin{aligned}
\mathscr{O}_{X}^{\mathrm{E}} & \stackrel{\sim}{\longrightarrow} \mathscr{I h o m}^{+}\left(\mathbb{C}_{\{t \geq 0\}}, \mathscr{O}_{X}^{\mathrm{E}}\right) \\
& \stackrel{\sim}{ } \operatorname{Ihom}^{+}\left(\mathbb{C}_{\{t \geq a\}}, \mathscr{O}_{X}^{\mathrm{E}}\right) \quad \text { for any } a \geq 0 .
\end{aligned}
$$

In particular, $\mathscr{O}_{X}^{\mathrm{E}}$ is a stable object in $\mathrm{E}^{\mathrm{b}}\left(\mathrm{I} \mathscr{D}_{X}\right)$.

As a consequence of Proposition 7.2.5 and Proposition 6.5.3, we get the following result.

Corollary 7.2.6. There are isomorphisms in $\mathrm{E}^{\mathrm{b}}\left(\mathrm{I} \mathscr{D}_{X}\right)$

$$
\mathscr{O}_{X}^{\mathrm{E}} \simeq \mathscr{I} h o m^{+}\left(\mathbb{C}_{X}^{\mathrm{E}}, \mathscr{O}_{X}^{\mathrm{E}}\right) \simeq \mathbb{C}_{X}^{\mathrm{E}} \stackrel{+}{\otimes} \mathscr{O}_{X}^{\mathrm{E}}
$$


Then, using the isomorphisms

$$
\begin{aligned}
& \mathscr{I h o m}^{\mathrm{E}}\left(\mathbb{C}_{X}^{\mathrm{E}}, \mathscr{O}_{X}^{\mathrm{E}}\right) \simeq \mathscr{I h o m}^{\mathrm{E}}\left(\mathbb{C}_{X}^{\mathrm{E}}, \mathscr{I h o m}^{+}\left(\mathbb{C}_{X}^{\mathrm{E}}, \mathscr{O}_{X}^{\mathrm{E}}\right)\right) \\
& \simeq \mathscr{I h o m}^{\mathrm{E}}\left(\mathbb{C}_{X}^{\mathrm{E}}{ }^{+} \mathbb{C}_{X}^{\mathrm{E}}, \mathscr{O}_{X}^{\mathrm{E}}\right) \\
& \simeq \mathscr{I h o m}^{\mathrm{E}}\left(\mathbb{C}_{\{t=0\}} \stackrel{+}{\otimes} \mathbb{C}_{X}^{\mathrm{E}}, \mathscr{O}_{X}^{\mathrm{E}}\right) \\
& \simeq \mathscr{I h o m}^{\mathrm{E}}\left(\mathbb{C}_{\{t=0\}}, \mathscr{O}_{X}^{\mathrm{E}}\right)
\end{aligned}
$$

and (7.2.2), one gets the isomorphism in $\mathrm{D}^{\mathrm{b}}\left(\mathrm{I} \mathscr{D}_{X}\right)$ :

$$
\mathscr{I h o m}^{\mathrm{E}}\left(\mathbb{C}_{X}^{\mathrm{E}}, \mathscr{O}_{X}^{\mathrm{E}}\right) \simeq \mathscr{O}_{X}^{\mathrm{t}} .
$$

\subsection{Enhanced de Rham and Sol functors}

For $\mathscr{M} \in \mathrm{D}^{\mathrm{b}}\left(\mathscr{D}_{X}\right)$, set

$$
\begin{aligned}
\mathcal{D R}_{X}^{\mathrm{E}}(\mathscr{M}) & :=\Omega_{X}^{\mathrm{E}} \stackrel{\mathrm{L}}{\mathscr{\otimes}_{\mathscr{D}_{X}}}, \mathscr{M}, \\
\mathcal{S}_{o l} \mathrm{E}(\mathscr{M}) & :=\operatorname{R} \mathscr{H}_{\mathscr{D}_{X}}^{\mathrm{E}}\left(\mathscr{M}, \mathscr{O}_{X}^{\mathrm{E}}\right) .
\end{aligned}
$$

We get functors

$$
\begin{aligned}
& \mathcal{D} \mathcal{R}_{X}^{\mathrm{E}}: \mathrm{D}^{\mathrm{b}}\left(\mathscr{D}_{X}\right) \rightarrow \mathrm{E}^{\mathrm{b}}\left(\mathrm{IC}_{X}\right), \\
& \mathcal{S}_{\text {o }}^{\mathrm{E}}: \mathrm{D}^{\mathrm{b}}\left(\mathscr{D}_{X}\right)^{\mathrm{op}} \rightarrow \mathrm{E}^{\mathrm{b}}\left(\mathrm{IC}_{X}\right) .
\end{aligned}
$$

Note that

$$
\operatorname{Sol}_{X}^{\mathrm{E}}(\mathscr{M}) \simeq \mathcal{D} \mathcal{R}_{X}^{\mathrm{E}}\left(\mathbb{D}_{X} \mathscr{M}\right)\left[-d_{X}\right] \quad \text { for } \mathscr{M} \in \mathrm{D}_{\text {coh }}^{\mathrm{b}}\left(\mathscr{D}_{X}\right) .
$$

By (7.2.3), we have for any $\mathscr{M} \in \mathrm{D}^{\mathrm{b}}\left(\mathscr{D}_{X}\right)$

$$
\begin{aligned}
& \mathcal{D} \mathcal{R}_{X}^{\mathrm{t}} \mathscr{M} \simeq \mathscr{I} \operatorname{hom}^{\mathrm{E}}\left(\mathbb{C}_{X}^{\mathrm{E}}, \mathcal{D} \mathcal{R}_{X}^{\mathrm{E}} \mathscr{M}\right), \\
& \mathcal{D} \mathcal{R}_{X} \mathscr{M} \simeq \mathscr{H} o m^{\mathrm{E}}\left(\mathbb{C}_{X}^{\mathrm{E}}, \mathcal{D} \mathcal{R}_{X}^{\mathrm{E}} \mathscr{M}\right) .
\end{aligned}
$$

By using Proposition 7.1.2, one can calculate explicitly $\mathcal{D} \mathcal{R}_{X}^{\mathrm{E}}(\mathscr{M})$ when $\mathscr{M}$ is an exponential D-module.

Proposition 7.3.1. Let $Y \subset X$ be a closed complex analytic hypersurface, and set $U=X \backslash Y$. For $\varphi \in \mathscr{O}_{X}(* Y)$, there are isomorphisms

$$
\begin{aligned}
\mathcal{D R}_{X}^{\mathrm{E}}\left(\mathscr{E}_{U \mid X}^{\varphi}\right) & \simeq \operatorname{R} \mathscr{I h o m}\left(\pi^{-1} \mathbb{C}_{U}, \underset{c \rightarrow \infty}{\stackrel{\lim }{\longrightarrow}} \mathbb{C}_{\{t \geq \operatorname{Re} \varphi+c\}}\right) \\
& \simeq \mathbb{C}_{X}^{\mathrm{E}} \stackrel{+}{\otimes} \operatorname{R} \mathscr{I h o m}\left(\pi^{-1} \mathbb{C}_{U}, \mathbb{C}_{\{t=\operatorname{Re} \varphi\}}\right) .
\end{aligned}
$$


The next results are easy consequences of Theorem 3.1.1, Corollary 3.1.2, Corollary 3.1.6 and Corollary 3.1.7.

Theorem 7.3.2. Let $f: X \rightarrow Y$ be a morphism of complex manifolds.

(i) There is an isomorphism in $\mathrm{E}^{\mathrm{b}}\left(\mathrm{I}\left(f^{-1} \mathscr{D}_{Y}\right)\right)$

$$
\mathrm{E} f^{!} \mathscr{O}_{Y}^{\mathrm{E}}\left[d_{Y}\right] \simeq \mathscr{D}_{Y \leftarrow X} \stackrel{\mathrm{L}}{\mathscr{D}_{X}} \mathscr{O}_{X}^{\mathrm{E}}\left[d_{X}\right] .
$$

(ii) For any $\mathscr{N} \in \mathrm{D}^{\mathrm{b}}\left(\mathscr{D}_{Y}\right)$ there is an isomorphism in $\mathrm{E}^{\mathrm{b}}\left(\mathrm{IC}_{X}\right)$

$$
\mathcal{D} \mathcal{R}_{X}^{\mathrm{E}}\left(\mathrm{D} f^{*} \mathscr{N}\right)\left[d_{X}\right] \simeq \mathrm{E} f^{!} \mathcal{D} \mathcal{R}_{Y}^{\mathrm{E}}(\mathscr{N})\left[d_{Y}\right] .
$$

(iii) Let $\mathscr{M} \in \mathrm{D}_{\text {good }}^{\mathrm{b}}\left(\mathscr{D}_{X}\right)$, and assume that $\operatorname{Supp}(\mathscr{M})$ is proper over $Y$. Then, there are isomorphisms in $\mathrm{E}^{\mathrm{b}}\left(\mathrm{IC}_{Y}\right)$

$$
\begin{aligned}
& \mathcal{D} \mathcal{R}_{Y}^{\mathrm{E}}\left(\mathrm{D} f_{*} \mathscr{M}\right) \simeq \mathrm{E}_{*} \mathcal{D} \mathcal{R}_{X}^{\mathrm{E}}(\mathscr{M}), \\
& \mathrm{D} f_{*}\left(\mathscr{O}_{X}^{\mathrm{E}} \stackrel{\mathrm{D}}{\otimes} \mathscr{M}\right) \simeq \mathscr{O}_{Y}^{\mathrm{E}} \otimes \stackrel{\mathrm{D}}{\otimes} f_{*} \mathscr{M} .
\end{aligned}
$$

\subsection{Ordinary linear differential equations and Stokes phenomena}

Let us recall the local theory of ordinary linear differential equations. Let $X \subset \mathbb{C}$ be open with $0 \in X$ and let $\mathscr{M}$ be a holonomic $\mathscr{D}_{X}$-module such that $\operatorname{SingSupp}(\mathscr{M}) \subset\{0\}$ and $\mathscr{M} \simeq \mathscr{M}(*\{0\})$. Then $\mathscr{M}$ is a locally free $\mathscr{O}_{X}(*\{0\})$-module of finite rank. Let us take a system of generators $\left(u_{1}, \ldots, u_{r}\right)$ of $\mathscr{M}$ as an $\mathscr{O}_{X}(*\{0\})$-module on a neighborhood of 0 . Then, setting $\overrightarrow{\boldsymbol{u}}$ the column vector consisting of these generators, we have

$$
\frac{d}{d z} \overrightarrow{\boldsymbol{u}}=\boldsymbol{A}(z) \overrightarrow{\boldsymbol{u}}
$$

for an $(r \times r)$-matrix $\boldsymbol{A}(z)$ whose components are in $\mathscr{O}_{X}(*\{0\})$. Then for any $\mathscr{D}_{X}$-module $\mathscr{L}$ such that $\mathscr{L} \simeq \mathscr{L}(*\{0\})$, we have

$$
\mathscr{H}_{0 m_{\mathscr{D}_{X}}}(\mathscr{M}, \mathscr{L})=\left\{\vec{u} \in \mathscr{L}^{r} ; \vec{u} \text { satisfies equation (7.4.2) below }\right\}
$$

where we associate to $\vec{u}$ the morphism from $\mathscr{M}$ to $\mathscr{L}$ defined by $\overrightarrow{\boldsymbol{u}} \mapsto \vec{u}$. Here

$$
\frac{d}{d z} \vec{u}=\boldsymbol{A}(z) \vec{u} .
$$

Now we have the following results on the solutions of the ordinary linear differential equation (7.4.2). 
(i) there exist linearly independent $r$ formal (column) solutions $\widehat{\boldsymbol{u}}_{j}(j=$ $1, \ldots r)$ of $(7.4 .2)$ with the form

$$
\widehat{\boldsymbol{u}}_{j}=\mathrm{e}^{\varphi_{j}(z)} z^{\lambda_{j}} \sum_{k=0}^{r-1} \vec{a}_{j, k}(z)(\log z)^{k},
$$

where $m \in \mathbb{Z}_{>0}, \varphi_{j}(z) \in z^{-1 / m} \mathbb{C}\left[z^{-1 / m}\right], \lambda_{j} \in \mathbb{C}$, and

$$
\vec{a}_{j, k}(z)=\sum_{n \in m^{-1} \mathbb{Z}_{\geq 0}} \vec{a}_{j, k, n} z^{n} \in \mathbb{C}\left[\left[z^{1 / m}\right]\right]^{r} \text { with } \vec{a}_{j, k, n} \in \mathbb{C}^{r},
$$

(ii) for any $\theta_{0} \in \mathbb{R}$ and each $j=1, \ldots, r$, there exist an angular neighborhood

$$
D_{\theta_{0}}=\left\{z=r \mathrm{e}^{i \theta} ;\left|\theta-\theta_{0}\right|<\varepsilon \text { and } 0<r<\delta\right\}
$$

for sufficiently small $\varepsilon, \delta>0$ and holomorphic (column) solution $\boldsymbol{u}_{j} \in$ $\mathscr{O}_{X}\left(D_{\theta_{0}}\right)^{r}$ of (7.4.2) defined on $D_{\theta_{0}}$ such that

$$
\boldsymbol{u}_{j} \sim \widehat{\boldsymbol{u}}_{j}
$$

in the sense that, for any $N>0$, there exists $C>0$ such that

$$
\left|\boldsymbol{u}_{j}(z)-\widehat{\boldsymbol{u}}_{j}^{N}(z)\right| \leq C\left|\mathrm{e}^{\varphi_{j}(z)} z^{\lambda_{j}+N}\right|=C \mathrm{e}^{\operatorname{Re}\left(\varphi_{j}(z)\right)}\left|z^{\lambda_{j}+N}\right|,
$$

where $\widehat{\boldsymbol{u}}_{j}^{N}(z)$ is the finite partial sum

$$
\widehat{\boldsymbol{u}}_{j}^{N}(z)=\mathrm{e}^{\varphi_{j}(z)} z^{\lambda_{j}} \sum_{k=0}^{r-1} \sum_{\substack{n \in m^{-1} \mathbb{Z}_{\geq 0}, n \leq N}} \vec{a}_{j, k, n} z^{n}(\log z)^{k} .
$$

Here we choose branches of $z^{1 / m}$ and $\log z$ on $D_{\theta_{0}}$.

Note that a holomorphic solution $\boldsymbol{u}_{j}$ is not uniquely determined by the formal solution $\widehat{\boldsymbol{u}}_{j}$. In fact, $\boldsymbol{u}_{j}+\sum_{k} c_{k} \boldsymbol{u}_{k}$ also satisfies the same estimate (7.4.3) whenever

$$
\operatorname{Re}\left(\varphi_{k}(z)\right)<\operatorname{Re}\left(\varphi_{j}(z)\right) \text { on } D_{\theta_{0}} \text { if } c_{k} \neq 0 .
$$


We can interpret these results as follows. Let $\varpi: \widetilde{X} \rightarrow X$ be the real blow up of $X$ along $\{0\}$ defined in $\S 4.2$. Then $\mathrm{e}^{-\varphi_{j}(z)} \boldsymbol{u}_{j}$ gives a section of $\left(\mathcal{A}_{\tilde{X}}\right)^{r}$ on a neighborhood of $\mathrm{e}^{i \theta_{0}} \in \varpi^{-1}(0)$. Define the $\mathscr{D}_{\tilde{X}}^{\mathcal{A}}$-module

$$
\mathscr{L}_{j}:=\mathscr{D} \mathcal{X}^{\mathcal{A}} \mathrm{e}^{\varphi_{j}(z)}=\mathscr{D} \mathcal{A} / \mathscr{D} \mathcal{A}\left(\frac{d}{d z}-\varphi_{j}^{\prime}(z)\right) .
$$

Here we take a branch of $\varphi_{j}$ on a domain and $\mathscr{L}_{j}$ is defined on such a domain.

Then $\left(\mathrm{e}^{-\varphi_{j}(z)} \boldsymbol{u}_{j}\right) \mathrm{e}^{\varphi_{j}} \in\left(\mathscr{L}_{j}\right)^{r}$ is a solution of equation (7.4.2), and hence (7.4.1) defines a morphism of $\mathscr{D} \tilde{X}^{\mathcal{A}}$-modules

$$
\mathscr{M}^{\mathcal{A}} \rightarrow \mathscr{L}_{j}
$$

Collecting such a morphism for all $j$, we obtain an isomorphism defined on a neighborhood of $\mathrm{e}^{i \theta_{0}} \in \varpi^{-1}(0)$ :

$$
\mathscr{M}^{\mathcal{A}} \stackrel{\sim}{\longrightarrow} \bigoplus_{j=1}^{r} \mathscr{L}_{j}
$$

Note that

$$
\varpi_{*} \mathscr{M}^{\mathcal{A}} \simeq \mathscr{M}
$$

However, these isomorphisms (7.4.4) are not globally defined. That is, $\mathscr{M}^{\mathcal{A}}$ is only locally isomorphic to $\bigoplus_{j=1}^{r} \mathscr{L}_{j}$. We have

$$
\left.\mathscr{H}_{o m_{\mathscr{D}_{\bar{X}}^{\mathcal{A}}}}\left(\mathscr{L}_{j}, \mathscr{L}_{j^{\prime}}\right)\right|_{\varpi^{-1}(0)} \simeq \mathbb{C}_{U_{j, j^{\prime}}} \subset \mathbb{C}_{\varpi^{-1}(0)},
$$

where

$$
U_{j, j^{\prime}}=\left\{p \in \varpi^{-1}(0) ; \begin{array}{l}
\operatorname{Re}\left(\varphi_{j}(z)\right) \leq \operatorname{Re}\left(\varphi_{j^{\prime}}(z)\right) \text { on } U \cap \tilde{X}^{>0} \text { for } \\
\text { a neighborhood } U \text { of } p
\end{array}\right.
$$

Indeed, any morphism $\left.f \in \mathscr{C}_{0 m_{\mathscr{D}_{\mathcal{X}}^{\mathcal{A}}}}\left(\mathscr{L}_{j}, \mathscr{L}_{j^{\prime}}\right)\right|_{\varpi^{-1}(0)}$ should have the form $\mathrm{e}^{\varphi_{j}} \mapsto \mathrm{e}^{\varphi_{j}(z)-\varphi_{j^{\prime}}(z)} \mathrm{e}^{\varphi_{j^{\prime}}}$ up to a constant multiple, and hence $f$ is well-defined if and only if $\mathrm{e}^{\varphi_{j}(z)-\varphi_{j^{\prime}}(z)}$ is tempered. The last condition is equivalent to the condition in (7.4.6).

Hence the isomorphism class of a $\left.\mathscr{D} \mathcal{A}\right|_{\varpi^{-1}(0)}$-module $\mathscr{L}$ locally isomorphic to $\left.\bigoplus_{j=1}^{r} \mathscr{L}_{j}\right|_{\varpi^{-1}(0)}$ is determined by a topological data, the so-called Stokes matrices. 
Assuming that $m=1$ for the sake of simplicity, let us explain them more precisely. Let $\mathscr{L}$ be a $\left.\mathscr{D} \hat{\mathcal{A}}\right|_{\varpi^{-1}(0)}$-module locally isomorphic to $\left.\bigoplus_{j=1}^{r} \mathscr{L}_{j}\right|_{\varpi^{-1}(0)}$. We identify $\varpi^{-1}(0)$ with $\mathbb{R} / 2 \pi \mathbb{Z}$ by $\mathbb{R} / 2 \pi \mathbb{Z} \ni \theta \mapsto \mathrm{e}^{i \theta} \in \varpi^{-1}(0)$. Let us take $\left\{\theta_{1}, \ldots, \theta_{s}\right\}$ such that $s \geq 2, \theta_{0}<\theta_{1}<\cdots<\theta_{s-1}<\theta_{s}$ and

$$
\varpi^{-1}(0) \bigcap \bigcup_{\substack{1 \leq j, j^{\prime} \leq r, \varphi_{j} \neq \varphi_{j^{\prime}}}} \overline{\left\{z \in \widetilde{X}^{>0} ; \operatorname{Re} \varphi_{j}(z)=\operatorname{Re} \varphi_{j^{\prime}}(z)\right\}} \subset\left\{\theta_{1}, \ldots, \theta_{s}\right\} .
$$

Here we set $\theta_{k+l s}=\theta_{k}+2 \pi l$ for $1 \leq k \leq s$ and $l \in \mathbb{Z}$. Set $V_{k}=$ $\left\{\theta ; \theta_{k-1}<\theta<\theta_{k+1}\right\}$ and $W_{k}=\left\{\theta ; \theta_{k}<\theta<\theta_{k+1}\right\}=V_{k} \cap V_{k+1}$. Then we have $\varpi^{-1}(0)=\bigcup_{1 \leq k \leq s} V_{k}$. Note that for any $j, j^{\prime} \in\{1, \ldots, r\}$ and $k \in\{1, \ldots, s\}$, we have either $W_{k} \cap U_{j, j^{\prime}}=\emptyset$ or $W_{k} \subset U_{j, j^{\prime}}$. In particular, (7.4.5) implies that $\left.\mathscr{H}_{0 m_{\mathscr{D} \mathcal{X}}}\left(\mathscr{L}_{j}, \mathscr{L}_{j^{\prime}}\right)\right|_{W_{k}}$ is a constant sheaf.

Hence, any isomorphism $\left.\mathscr{L} \stackrel{\sim}{\longrightarrow} \bigoplus_{j=1}^{r} \mathscr{L}_{j}\right|_{\varpi^{-1}(0)}$ defined on a neighborhood of $\theta_{k}$ can be extended to an isomorphism defined on $V_{k}$, and we have an isomorphism

$$
\psi_{k}:\left.\left.\mathscr{L}\right|_{V_{k}} \stackrel{\sim}{\longrightarrow} \bigoplus_{j=1}^{r} \mathscr{L}_{j}\right|_{V_{k}}
$$

Let us set

$$
\xi_{k}=\psi_{k+1} \circ \psi_{k}^{-1}:\left.\left.\bigoplus_{j=1}^{r} \mathscr{L}_{j}\right|_{W_{k}} \stackrel{\sim}{\longrightarrow} \bigoplus_{j=1}^{r} \mathscr{L}_{j}\right|_{W_{k}} .
$$

Then $\mathscr{L}$ is obtained by patching $\left.\bigoplus_{j=1}^{r} \mathscr{L}_{j}\right|_{V_{k}}$ by the $\xi_{k}$ 's. Each isomorphism $\xi_{k}$ is given by the matrix $S_{k}=\left(s_{k ; i^{\prime}, i}\right)_{1 \leq i, i^{\prime} \leq r} \in \mathrm{GL}_{r}(\mathbb{C})$. Here $s_{k ; i^{\prime}, i} \in \mathbb{C}$ is given by the morphism

$$
\left.\left.\left.\left.\mathscr{L}_{i}\right|_{W_{k}} \longrightarrow \bigoplus_{j=1}^{r} \mathscr{L}_{j}\right|_{W_{k}} \underset{\xi_{k}}{\sim} \bigoplus_{j=1}^{r} \mathscr{L}_{j}\right|_{W_{k}} \longrightarrow \mathscr{L}_{i^{\prime}}\right|_{W_{k}}
$$

through

$$
\Gamma\left(W_{k} ;\left.\mathscr{H}_{\mathscr{D}_{\hat{X}}^{\mathcal{A}}}\left(\mathscr{L}_{i}, \mathscr{L}_{i^{\prime}}\right)\right|_{\varpi^{-1}(0)}\right) \simeq \Gamma\left(W_{k} ; \mathbb{C}_{U_{i, i^{\prime}}}\right) \subset \Gamma\left(W_{k} ; \mathbb{C}_{\varpi^{-1}(0)}\right) \simeq \mathbb{C}
$$

due to (7.4.5). Hence, we have $s_{k ; i^{\prime}, i}=0$ if $W_{k} \not \subset U_{i, i^{\prime}}$.

The matrices $\left\{S_{k}\right\}_{1 \leq k \leq s}$ are called the Stokes matrices. Conversely, for a given family of matrices $\left\{S_{k}\right\}_{1 \leq k \leq s}$, we can find a $\mathscr{D} \tilde{X}_{\varpi^{-1}(0)}$-module $\mathscr{L}$ 
locally isomorphic to $\left.\bigoplus_{j=1}^{r} \mathscr{L}_{j}\right|_{\varpi^{-1}(0)}$ by patching $\left.\bigoplus_{j=1}^{r} \mathscr{L}_{j}\right|_{V_{k}}$ by $\left\{S_{k}\right\}_{1 \leq k \leq s}$. Then $\mathscr{M}$ is recovered from $\mathscr{L}$ by $\mathscr{M} \simeq \varpi_{*} \mathscr{L}$.

\subsection{Normal form}

The results in $\S 7.4$ are generalized to higher dimensions by T. Mochizuki ([Mo 09, Mo11]) and K. S. Kedlaya ([Ke10, Ke11]). In this subsection, we collect some of their results that we shall need.

Let $X$ be a complex manifold and $D \subset X$ a normal crossing divisor. We shall use the notations introduced in $\S 4.2$ : in particular the real blow up $\varpi: \widetilde{X} \rightarrow X$ and the notation $\mathscr{M}^{\mathcal{A}}$ of (4.2.10).

Definition 7.5.1. We say that a holonomic $\mathscr{D}_{X}$-module $\mathscr{M}$ has a normal form along $D$ if

(i) $\mathscr{M} \simeq \mathscr{M}(* D)$,

(ii) $\operatorname{SingSupp}(\mathscr{M}) \subset D$,

(iii) for any $x \in \varpi^{-1}(D) \subset \widetilde{X}$, there exist an open neighborhood $U \subset X$ of $\varpi(x)$ and finitely many $\varphi_{i} \in \Gamma\left(U ; \mathscr{O}_{X}(* D)\right)$ such that

$$
\left.\left.\left(\mathscr{M}^{\mathcal{A}}\right)\right|_{V} \simeq\left(\bigoplus_{i}\left(\mathscr{E}_{U \backslash D \mid U}^{\varphi_{i}}\right)^{\mathcal{A}}\right)\right|_{V}
$$

for some open neighborhood $V$ of $x$ with $V \subset \varpi^{-1}(U)$.

A ramification of $X$ along $D$ on a neighborhood $U$ of $x \in D$ is a finite map

$$
p: X^{\prime} \rightarrow U
$$

of the form $p\left(z^{\prime}\right)=\left(z_{1}^{\prime m_{1}}, \ldots, z_{r}^{\prime m_{r}}, z_{r+1}^{\prime}, \ldots, z_{n}^{\prime}\right)$ for some $\left(m_{1}, \ldots, m_{r}\right) \in$ $\left(\mathbb{Z}_{>0}\right)^{r}$. Here $\left(z_{1}^{\prime}, \ldots, z_{n}^{\prime}\right)$ is a local coordinate system on $X^{\prime},\left(z_{1}, \ldots, z_{n}\right)$ a local coordinate system on $X$ such that $D=\left\{z_{1} \cdots z_{r}=0\right\}$.

Definition 7.5.2. We say that a holonomic $\mathscr{D}_{X}$-module $\mathscr{M}$ has a quasinormal form along $D$ if it satisfies (i) and (ii) in Definition 7.5.1, and if for any $x \in D$ there exists a ramification $p: X^{\prime} \rightarrow U$ on a neighborhood $U$ of $x$ such that $\mathrm{D} p^{*}\left(\left.\mathscr{M}\right|_{U}\right)$ has a normal form along $p^{-1}(D \cap U)$.

Remark 7.5.3. In the above definition, $\mathrm{D} p^{*}\left(\left.\mathscr{M}\right|_{U}\right)$ as well as $\mathrm{D} p_{*} \mathrm{D} p^{*}\left(\left.\mathscr{M}\right|_{U}\right)$ is concentrated in degree zero and $\left.\mathscr{M}\right|_{U}$ is a direct summand of $\mathrm{D} p_{*} \mathrm{D} p^{*}\left(\left.\mathscr{M}\right|_{U}\right)$. 
The next result is an essential tool in the study of holonomic D-module and is easily deduced from the fundamental work of Mochizuki [Mo 09, Mo 11] (see also Sabbah [Sa 00] for preliminary results and see Kedlaya [Ke10, Ke11] for the analytic case).

Theorem 7.5.4. Let $X$ be a complex manifold, $\mathscr{M}$ a holonomic $\mathscr{D}_{X}$-module and $x \in X$. Then there exist an open neighborhood $U$ of $x$, a closed analytic hypersurface $Y \subset U$, a complex manifold $X^{\prime}$ and a projective morphism $f: X^{\prime} \rightarrow U$ such that

(i) $\operatorname{SingSupp}(\mathscr{M}) \cap U \subset Y$,

(ii) $D:=f^{-1}(Y)$ is a normal crossing divisor of $X^{\prime}$,

(iii) $f$ induces an isomorphism $X^{\prime} \backslash D \rightarrow U \backslash Y$,

(iv) $\left(\mathrm{D} f^{*} \mathscr{M}\right)(* D)$ has a quasi-normal form along $D$.

Remark that, under assumption (iii), $\left(\mathrm{D} f^{*} \mathscr{M}\right)(* D)$ is concentrated in degree zero.

Using Theorem 7.5.4, one easily deduces the next lemma.

Lemma 7.5.5. Let $P_{X}(\mathscr{M})$ be a statement concerning a complex manifold $X$ and a holonomic object $\mathscr{M} \in \mathrm{D}_{\mathrm{hol}}^{\mathrm{b}}\left(\mathscr{D}_{X}\right)$. Consider the following conditions.

(a) Let $X=\bigcup_{i \in I} U_{i}$ be an open covering. Then $P_{X}(\mathscr{M})$ is true if and only if $P_{U_{i}}\left(\left.\mathscr{M}\right|_{U_{i}}\right)$ is true for any $i \in I$.

(b) If $P_{X}(\mathscr{M})$ is true, then $P_{X}(\mathscr{M}[n])$ is true for any $n \in \mathbb{Z}$.

(c) Let $\mathscr{M}^{\prime} \rightarrow \mathscr{M} \rightarrow \mathscr{M}^{\prime \prime} \stackrel{+1}{\longrightarrow}$ be a distinguished triangle in $\mathrm{D}_{\mathrm{hol}}^{\mathrm{b}}\left(\mathscr{D}_{X}\right)$. If $P_{X}\left(\mathscr{M}^{\prime}\right)$ and $P_{X}\left(\mathscr{M}^{\prime \prime}\right)$ are true, then $P_{X}(\mathscr{M})$ is true.

(d) Let $\mathscr{M}$ and $\mathscr{M}^{\prime}$ be holonomic $\mathscr{D}_{X}$-modules. If $P_{X}\left(\mathscr{M} \oplus \mathscr{M}^{\prime}\right)$ is true, then $P_{X}(\mathscr{M})$ is true.

(e) Let $f: X \rightarrow Y$ be a projective morphism and $\mathscr{M}$ a good holonomic $\mathscr{D}_{X^{-}}$ module. If $P_{X}(\mathscr{M})$ is true, then $P_{Y}\left(\mathrm{D} f_{*} \mathscr{M}\right)$ is true.

(f) If $\mathscr{M}$ is a holonomic $\mathscr{D}_{X}$-module with a normal form along a normal crossing divisor of $X$, then $P_{X}(\mathscr{M})$ is true. 
If conditions (a)-(f) are satisfied, then $P_{X}(\mathscr{M})$ is true for any complex manifold $X$ and any $\mathscr{M} \in \mathrm{D}_{\text {hol }}^{\mathrm{b}}\left(\mathscr{D}_{X}\right)$.

Sketch of the proof. The proof is similar to the regular case (Lemma 4.1.4).

We shall only prove here that $P_{X}(\mathscr{M})$ is true for any holonomic $\mathscr{D}_{X^{-}}$ module $\mathscr{M}$ which has a quasi-normal form along a normal crossing divisor $D$.

Let $p: X^{\prime} \rightarrow U$ be as in Definition 7.5.2. Then $\mathrm{D} p^{*}\left(\left.\mathscr{M}\right|_{U}\right)$ has a normal form along $p^{-1}(D \cap U)$. Hence $P_{X^{\prime}}\left(\mathrm{D} p^{*}\left(\left.\mathscr{M}\right|_{U}\right)\right)$ is true by hypothesis (f). Hence $P_{U}\left(\mathrm{D} p_{*} \mathrm{D} p^{*}\left(\left.\mathscr{M}\right|_{U}\right)\right)$ is true by hypothesis (e). We have a chain of morphisms

$$
\left.\left.\mathscr{M}\right|_{U} \rightarrow \mathrm{D} p_{*} \mathrm{D} p^{*}\left(\left.\mathscr{M}\right|_{U}\right) \rightarrow \mathscr{M}\right|_{U},
$$

whose composition is equal to $m \mathrm{id}_{\mathscr{M}}$ where $m$ is the number of the generic fiber of $p$. Hence $\left.\mathscr{M}\right|_{U}$ is a direct summand of $\mathrm{D} p_{*} \mathrm{D} p^{*}\left(\left.\mathscr{M}\right|_{U}\right)$. Then, hypothesis (d) implies that $P_{U}\left(\left.\mathscr{M}\right|_{U}\right)$ is true.

Q.E.D.

\subsection{Enhanced de Rham functor on the real blow up}

By Lemma 7.5.5, many statements on holonomic D-modules can be reduced to the normal form case. In order to investigate this case, we shall introduce the enhanced de Rham functor on the real blow up.

Let $D$ be a normal crossing divisor of a complex manifold $X$ and let $\varpi: \widetilde{X} \rightarrow X$ be the real blow up of $X$ along $D$ as in $\S 4.2$. Let $j: \widetilde{X} \times \mathbb{R}_{\infty} \rightarrow$ $\widetilde{X}^{\text {tot }} \times \mathbb{P}^{1}(\mathbb{R})$ be the canonical morphism of bordered spaces. Similarly to (4.2.3), we set

$$
\mathcal{D} b_{\tilde{X} \times \mathbb{R}_{\infty}}^{\mathrm{t}}:=j^{-1} \mathscr{I h o m}\left(\mathbb{C}_{\tilde{X}>0 \times \mathbb{R}}, \mathcal{D} b_{\widetilde{X}^{\mathrm{tot}} \times \mathbb{P}^{1}(\mathbb{R})}^{\mathrm{t}}\right) .
$$

Then as in in Definition 7.2.1 one denotes by $\mathcal{D} b_{\tilde{X}}^{\top} \in \mathrm{D}^{\mathrm{b}}\left(\mathbb{I}_{\tilde{X} \times \mathbb{R}_{\infty}}\right)$ the complex, concentrated in degree -1 and 0 :

$$
\mathcal{D} b_{\widetilde{X}}^{\top}:=\mathcal{D} b_{\widetilde{X} \times \mathbb{R}_{\infty}}^{\mathrm{t}} \stackrel{\partial_{t}-1}{\longrightarrow} \mathcal{D} b_{\widetilde{X} \times \mathbb{R}_{\infty}}^{\mathrm{t}},
$$

and finally as in Definition 7.2.3, one sets

$$
\begin{aligned}
\mathscr{O}_{\tilde{X}}^{\mathrm{E}} & =\mathrm{R} \mathscr{H} o m_{\pi^{-1} \mathscr{D}_{X}}\left(\pi^{-1} \mathscr{O}_{X^{c}}, \mathcal{D} b_{\tilde{X}}^{\mathrm{T}}\right), \\
\Omega_{\tilde{X}}^{\mathrm{E}} & =\tilde{\pi}^{-1} \Omega_{X} \otimes_{\tilde{\pi}^{-1} \mathscr{O}_{X}} \mathscr{O}_{\tilde{X}}^{\mathrm{E}},
\end{aligned}
$$


where $\tilde{\pi}: \tilde{X} \times \mathbb{R}_{\infty} \rightarrow X$ is the canonical morphism. We regard them as objects of $\mathrm{E}^{\mathrm{b}}\left(\mathrm{I} \mathscr{D}_{\tilde{X}}\right)$ and $\mathrm{E}^{\mathrm{b}}\left(\mathrm{I} \mathscr{D}_{\tilde{X}}^{\text {op }}\right)$, respectively. Then

$$
\begin{array}{ll}
\mathscr{O}_{\tilde{X}}^{\mathrm{E}} \simeq \mathrm{E} \varpi^{!} \mathscr{O}_{X}^{\mathrm{E}}(* D) & \text { in } \mathrm{E}^{\mathrm{b}}\left(\mathrm{I}\left(\varpi^{-1} \mathscr{D}_{X}\right)\right), \\
\mathrm{E}_{*} \mathscr{O}_{\tilde{X}}^{\mathrm{E}} \simeq \mathscr{O}_{X}^{\mathrm{E}}(* D) & \text { in } \mathrm{E}^{\mathrm{b}}\left(\mathrm{I}_{X}\right),
\end{array}
$$

where

$$
\mathscr{O}_{X}^{\mathrm{E}}(* D):=\mathscr{O}_{X}^{\mathrm{E}} \stackrel{\mathrm{D}}{\otimes} \mathscr{O}_{X}(* D) \simeq \mathrm{R} \mathscr{I h o m}\left(\pi^{-1} \mathbb{C}_{X \backslash D}, \mathscr{O}_{X}^{\mathrm{E}}\right) .
$$
by

Then, for $\mathscr{N} \in \mathrm{D}^{\mathrm{b}}(\mathscr{D} \tilde{\mathcal{X}})$, we define the enhanced de Rham functor on $\widetilde{X}$

$$
\begin{aligned}
& \mathcal{D} \mathcal{R}_{\widetilde{X}}^{\mathrm{E}}(\mathscr{N})=\Omega_{\widetilde{X}}^{\mathrm{E}} \stackrel{\mathrm{L}}{\otimes_{\mathscr{D} \mathcal{A}}} \mathscr{N},
\end{aligned}
$$

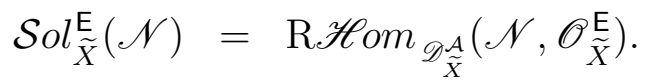

Then (7.6.2) and (7.6.3) imply that

$$
\begin{array}{ll}
\mathcal{D} \mathcal{R}_{\widetilde{X}}^{\mathrm{E}}\left(\mathscr{M}^{\mathcal{A}}\right) \simeq \mathrm{E} \varpi ! \mathcal{D} \mathcal{R}_{X}^{\mathrm{E}}(\mathscr{M}(* D)) & \text { in } \mathrm{E}^{\mathrm{b}}\left(\mathrm{I}_{\widetilde{X}}\right), \\
\mathrm{E} \varpi_{*} \mathcal{D} \mathcal{R}_{\widetilde{X}}^{\mathrm{E}}\left(\mathscr{M}^{\mathcal{A}}\right) \simeq \mathcal{D} \mathcal{R}_{X}^{\mathrm{E}}(\mathscr{M}(* D)) & \text { in } \mathrm{E}^{\mathrm{b}}\left(\mathrm{IC}_{X}\right) .
\end{array}
$$

for any $\mathscr{M} \in \mathrm{D}^{\mathrm{b}}\left(\mathscr{D}_{X}\right)$.

\subsection{De Rham functor: constructibility and duality}

Theorem 7.7.1. Let $\mathscr{M} \in \mathrm{D}_{\text {hol }}^{\mathrm{b}}\left(\mathscr{D}_{X}\right)$. Then $\mathcal{D R}_{X}^{\mathrm{E}}(\mathscr{M})$ and $\mathcal{S}_{\text {ol }}^{\mathrm{E}}(\mathscr{M})$ belong to $\mathrm{E}_{\mathbb{R}-c}^{\mathrm{b}}\left(\mathrm{IC}_{X}\right)$.

Sketch of the proof. Using Lemma 7.5.5, one reduces the proof to the case where $\mathscr{M}$ has a normal form along a normal crossing divisor $D$. Let $\varpi: \widetilde{X} \rightarrow$ $X$ be the real blow up along $D$.

Then, $\mathscr{M}^{\mathcal{A}}$ is locally isomorphic to a direct sum of $\left(\mathscr{E}_{U \backslash D \mid U}^{\varphi}\right)^{\mathcal{A}}$ with $\varphi \in$ $\Gamma\left(U ; \mathscr{O}_{X}(* D)\right)$. Since Proposition 7.3.1 implies that $\mathcal{D} \mathcal{R}_{\tilde{X}}^{\mathrm{E}}\left(\left(\mathscr{E}_{U \backslash D \mid U}^{\varphi}\right)^{\mathcal{A}}\right) \simeq$ $\mathrm{E} \varpi ! \mathcal{D} \mathcal{R}_{X}^{\mathrm{E}}\left(\mathscr{E}_{U \backslash D \mid U}^{\varphi}\right)$ is $\mathbb{R}$-constructible, $\mathcal{D} \mathcal{R}_{\tilde{X}}^{\mathrm{E}}\left(\mathscr{M}^{\mathcal{A}}\right)$ is $\mathbb{R}$-constructible. Hence $\mathcal{D} \mathcal{R}_{X}^{\mathrm{E}}(\mathscr{M}) \simeq \mathrm{E} \varpi_{*} \mathcal{D} \mathcal{R}_{\tilde{X}}^{\mathrm{E}}\left(\mathscr{M}^{\mathcal{A}}\right)$ is $\mathbb{R}$-constructible.

Q.E.D.

Corollary 7.7.2. For any $\mathscr{M} \in \mathrm{D}_{\text {hol }}^{\mathrm{b}}\left(\mathscr{D}_{X}\right)$ and $F \in \mathrm{D}_{\mathbb{R}-c}^{\mathrm{b}}\left(\mathbb{C}_{X}\right)$, the object $\mathrm{R} \mathscr{H}$ om $_{\mathscr{D}_{X}}\left(\mathscr{M}, \mathrm{R} \mathscr{H}\right.$ om $\left._{\mathrm{IC}_{X}}\left(F, \mathscr{O}_{X}^{\mathrm{t}}\right)\right)$ belongs to $\mathrm{D}_{\mathbb{R}_{-c}}^{\mathrm{b}}\left(\mathbb{C}_{X}\right)$. 
Proof. First note that

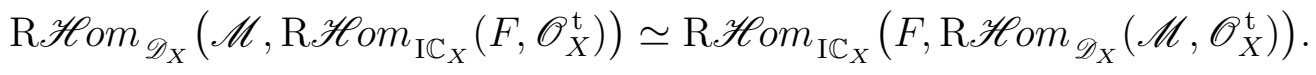

By Theorems 6.6.4 and 7.7.1, $\mathscr{H}_{o m}^{\mathrm{E}}\left(\pi^{-1} F \otimes \mathbb{C}_{X}^{E}, \mathcal{S}_{\text {ol }}^{\mathrm{E}}(\mathscr{M})\right)$ belongs to $\mathrm{D}_{\mathbb{R} \text {-c }}^{\mathrm{b}}\left(\mathbb{C}_{X}\right)$. Since $\mathscr{I} h_{o m}^{\mathrm{E}}\left(\mathbb{C}_{X}^{\mathrm{E}}, \mathscr{O}_{X}^{\mathrm{E}}\right) \simeq \mathscr{O}_{X}^{\mathrm{t}}$ by $(7.2 .3)$, we get

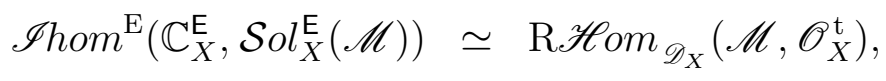

and

$$
\begin{aligned}
& \mathscr{H} o m^{\mathrm{E}}\left(\pi^{-1} F \otimes \mathbb{C}_{X}^{E}, \mathcal{S} o l_{X}^{\mathrm{E}}(\mathscr{M})\right) \simeq \operatorname{R} \mathscr{H} o m_{\mathrm{IC}_{X}}\left(F, \mathscr{I h o m}{ }^{\mathrm{E}}\left(\mathbb{C}_{X}^{E}, \mathcal{S}_{\text {ol }}^{\mathrm{E}}(\mathscr{M})\right)\right. \\
& \simeq \operatorname{R} \mathscr{H}_{0} m_{\mathscr{D}_{X}}\left(\mathscr{M}, \mathrm{R} \mathscr{H} o m_{\mathrm{IC}_{X}}\left(F, \mathscr{O}_{X}^{\mathrm{t}}\right)\right) \text {. }
\end{aligned}
$$

Q.E.D.

Lemma 7.7.3. Let $X_{1}$ and $X_{2}$ be a pair of complex manifolds. Let $\mathscr{M}_{j} \in$ $\mathrm{D}_{\text {hol }}^{\mathrm{b}}\left(\mathscr{D}_{X_{j}}\right)(j=1,2)$. Then we have a canonical isomorphism

$$
\mathcal{D} \mathcal{R}_{X_{1}}^{\mathrm{E}}\left(\mathscr{M}_{1}\right) \stackrel{+}{\otimes} \mathcal{D} \mathcal{R}_{X_{2}}^{\mathrm{E}}\left(\mathscr{M}_{2}\right) \stackrel{\sim}{\longrightarrow} \mathcal{D} \mathcal{R}_{X_{1} \times X_{2}}^{\mathrm{E}}\left(\mathscr{M}_{1} \stackrel{\mathrm{D}}{\otimes} \mathscr{M}_{2}\right)
$$

Sketch of the proof. Using Lemma 7.5.5, one reduces the proof to the case where $\mathscr{M}_{1}$ and $\mathscr{M}_{2}$ are exponential D-modules. In this case, the result follows from Proposition 7.3.1.

Q.E.D.

By using the functorial properties of the enhanced de Rham functor proved above, we can show that the enhanced de Rham functor commutes with duality.

Theorem 7.7.4. Let $\mathscr{M} \in \mathrm{D}_{\text {hol }}^{\mathrm{b}}\left(\mathscr{D}_{X}\right)$. Then, we have the isomorphism

$$
\mathcal{D} \mathcal{R}_{X}^{\mathrm{E}}\left(\mathbb{D}_{X} \mathscr{M}\right) \simeq \mathrm{D}_{X}^{\mathrm{E}} \mathcal{D} \mathcal{R}_{X}^{\mathrm{E}}(\mathscr{M})
$$

Note that $\mathcal{D} \mathcal{R}_{X}^{\mathrm{E}}\left(\mathbb{D}_{X} \mathscr{M}\right) \simeq \mathcal{S}_{\text {ol }}^{\mathrm{E}}(\mathscr{M})\left[d_{X}\right]$.

Idea of the proof.

Let $\mathscr{T}$ be a monoidal category with $\mathbf{1}$ as a unit object. Recall that a pair of objects $X$ and $Y$ are dual if and only if there exist morphisms

$$
\begin{aligned}
X \otimes Y & \stackrel{\varepsilon}{\longrightarrow} \mathbf{1}, \\
\mathbf{1} & \stackrel{\eta}{\longrightarrow} Y \otimes X
\end{aligned}
$$


such that the composition

$$
X \stackrel{X \otimes \eta}{\longrightarrow} X \otimes Y \otimes X \stackrel{\varepsilon \otimes X}{\longrightarrow} X
$$

is equal to $\mathrm{id}_{X}$ and

$$
Y \stackrel{\eta \otimes Y}{\longrightarrow} Y \otimes X \otimes Y \stackrel{Y \otimes \varepsilon}{\longrightarrow} Y
$$

is equal to $\mathrm{id}_{Y}$.

This criterion of duality has many variations.

Sheaf case: Let $M$ be a real analytic manifold, and let $F, G \in \mathrm{D}_{\mathbb{R}-\mathrm{c}}^{\mathrm{b}}\left(\mathbf{k}_{M}\right)$. Denote by $\Delta_{M}$ the diagonal subset of $M \times M$.

Now $F$ and $G$ are dual to each other, i.e., $G \simeq \mathrm{D}_{M} F$, if and only if there exist morphisms

$$
\begin{aligned}
F \otimes G & \stackrel{\varepsilon}{\longrightarrow} \omega_{\Delta_{M}}, \\
\mathbf{k}_{\Delta_{M}} & \stackrel{\eta}{\longrightarrow} G \otimes F
\end{aligned}
$$

such that the composition

$$
F \otimes \mathbf{k}_{\Delta_{M}} \stackrel{F \otimes \eta}{\longrightarrow} F \otimes G \otimes F \stackrel{\varepsilon \otimes F}{\longrightarrow} \omega_{\Delta_{M}} \otimes F
$$

is equal to $\mathrm{id}_{F}$ via isomorphism (7.7.8) below and

$$
\mathbf{k}_{\Delta_{M}} \otimes G \stackrel{\eta \otimes G}{\longrightarrow} G \otimes F \otimes G \stackrel{G \otimes \varepsilon}{\longrightarrow} G \otimes \omega_{\Delta_{M}}
$$

is equal to $\operatorname{id}_{G}$ via isomorphism (7.7.9) below.

Enhanced indsheaf case: Let $F$ and $G \in \mathrm{E}_{\mathbb{R}-c}^{\mathrm{b}}\left(\mathrm{Ik}_{M}\right)$. They are dual to each other, i.e., $G \simeq \mathrm{D}_{M}^{\mathrm{E}} F$, if and only if there exist morphisms

$$
\begin{array}{ccc}
F \stackrel{+}{\otimes} G \stackrel{\varepsilon}{\longrightarrow} \omega_{\Delta_{M}}^{\mathrm{E}}, \\
\mathbf{k}_{\Delta_{M}}^{\mathrm{E}} \stackrel{\eta}{\longrightarrow} G \stackrel{+}{\square} F
\end{array}
$$

such that the composition

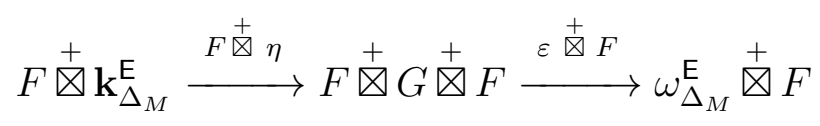


is equal to $\mathrm{id}_{F}$ via the enhanced version of isomorphism (7.7.8) below and

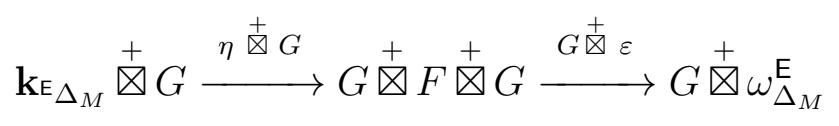

is equal to $\mathrm{id}_{G}$ via the enhanced version of isomorphism (7.7.9) below.

Holonomic D-module case: Let $X$ be a complex manifold and let $\delta: X \hookrightarrow$ $X \times X$ be the diagonal embedding. We set $\mathscr{B}_{\Delta_{X}}:=\mathrm{D} \delta_{*} \mathscr{O}_{X}$.

Let $\mathscr{M}, \mathscr{N} \in \mathrm{D}_{\text {hol }}^{\mathrm{b}}\left(\mathscr{D}_{X}\right)$. They are dual to each other, i.e., $\mathscr{N} \simeq \mathbb{D}_{X} \mathscr{M}$, if and only if there exist morphisms

$$
\begin{aligned}
& \mathscr{M} \unrhd \mathscr{\mathrm { D }} \stackrel{\varepsilon}{\longrightarrow} \mathscr{B}_{\Delta_{X}}\left[d_{X}\right], \\
& \mathscr{B}_{\Delta_{X}}\left[-d_{X}\right] \stackrel{\eta}{\longrightarrow} \mathscr{N} \stackrel{\mathrm{D}}{\otimes} \mathscr{M}
\end{aligned}
$$

such that the composition

$$
\mathscr{M} \stackrel{\mathrm{D}}{\otimes} \mathscr{B}_{\Delta_{X}}\left[-d_{X}\right] \stackrel{\mathscr{M} \otimes \eta}{\longrightarrow} \mathscr{M} \stackrel{\mathrm{D}}{\otimes} \mathscr{N} \stackrel{\mathrm{D}}{\otimes} \mathscr{M} \stackrel{\varepsilon \otimes}{\stackrel{\mathrm{D}}{M}} \longrightarrow \mathscr{B}_{\Delta_{X}}\left[d_{X}\right] \stackrel{\mathrm{D}}{\otimes} \mathscr{M}
$$

is equal to id $\mathscr{M}_{\mathcal{M}}$ via isomorphism (7.7.10) below and

$$
\mathscr{B}_{\Delta_{X}}\left[-d_{X}\right] \stackrel{\mathrm{D}}{\otimes} \mathscr{N} \stackrel{i \mathrm{D} \mathscr{N}}{\longrightarrow} \mathscr{N} \stackrel{\mathrm{D}}{\otimes} \mathscr{M} \stackrel{\mathrm{D}}{\otimes} \mathscr{M} \stackrel{\stackrel{\mathrm{D}}{\otimes} \varepsilon}{\longrightarrow} \mathscr{N} \stackrel{\mathrm{D}}{\otimes} \mathscr{B}_{\Delta_{X}}\left[d_{X}\right]
$$

is equal to id ${ }_{\mathscr{N}}$ via isomorphism (7.7.11) below.

Now we shall prove Theorem 7.7.4. Set $\mathscr{N}=\mathbb{D}_{X} \mathscr{M}$. Then we have morphisms as in (7.7.5) which satisfy the conditions that the compositions (7.7.6) and (7.7.7) are equal to id $\mathscr{M}_{\text {and }} \mathrm{id}_{\mathscr{N}}$, respectively. Now we shall apply the functor $\mathcal{D R}^{\mathrm{E}}$. Then we obtain morphisms as in (7.7.2) with $M=X_{\mathbb{R}}$, $\mathbf{k}=\mathbb{C}, F=\mathcal{D} \mathcal{R}_{X}^{\mathrm{E}}(\mathscr{M})$ and $G=\mathcal{D} \mathcal{R}_{X}^{\mathrm{E}}(\mathscr{N})$. Note that we have

$$
\mathcal{D} \mathcal{R}_{X \times X}^{\mathrm{E}}\left(\mathscr{B}_{\Delta_{X}}\left[-d_{X}\right]\right) \simeq \mathbb{C}_{\Delta_{X}}^{\mathrm{E}}, \quad \mathcal{D} \mathcal{R}_{X \times X}^{\mathrm{E}}\left(\mathscr{B}_{\Delta_{X}}\left[d_{X}\right]\right) \simeq \omega_{\Delta_{X}}^{\mathrm{E}} .
$$

By applying the functor $\mathcal{D} \mathcal{R}_{X \times X \times X}^{\mathrm{E}}$, the morphisms in (7.7.6) and (7.7.7) are sent to (7.7.3) and (7.7.4). Hence the compositions (7.7.3) and (7.7.4) are equal to $\operatorname{id}_{F}$ and $\operatorname{id}_{G}$, respectively. Thus we conclude that $G \simeq \mathrm{D}_{X}^{\mathrm{E}} F$.

Here is the lemma that we used in the course of the proof of Theorem 7.7.4. 
Lemma 7.7.5. Let $M$ be a real manifold and let $F, G \in \mathrm{D}^{\mathrm{b}}\left(\mathbf{k}_{M}\right)$. Then we have the isomorphisms

$$
\begin{aligned}
& \operatorname{Hom}_{\mathrm{D}^{\mathrm{b}}\left(\mathbf{k}_{M \times M \times M}\right)}\left(F \otimes \mathbf{k}_{\Delta_{M}}, \omega_{\Delta_{M}} \otimes G\right) \simeq \operatorname{Hom}_{\mathrm{D}^{\mathrm{b}}\left(\mathbf{k}_{M}\right)}(F, G), \\
& \operatorname{Hom}_{\mathrm{D}^{\mathrm{b}}\left(\mathbf{k}_{M \times M \times M}\right)}\left(\mathbf{k}_{\Delta_{M}} \otimes F, G \otimes \omega_{\Delta_{M}}\right) \simeq \operatorname{Hom}_{\mathrm{D}^{\mathrm{b}}\left(\mathbf{k}_{M}\right)}(F, G),
\end{aligned}
$$

where $\Delta_{M} \subset M \times M$ is the diagonal subset.

Proof. Define the maps $p_{i_{1}, \ldots, i_{n}}$ by $p_{i_{1}, \ldots, i_{n}}\left(x_{1}, \ldots, x_{m}\right)=\left(x_{i_{1}}, \ldots, x_{i_{n}}\right)$. Then we have a commutative diagram

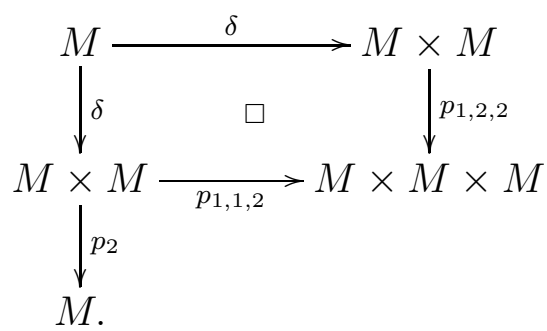

In the sequel, we write for short Hom instead of $\operatorname{Hom}_{\mathbf{k}_{N}}$ with $N=M, M \times$ $M \times M$. Then we have

$$
\begin{aligned}
\operatorname{Hom}\left(F \otimes \mathbf{k}_{\Delta_{M}}, \omega_{\Delta_{M}} \otimes G\right) & \simeq \operatorname{Hom}\left(\mathrm{R} p_{1,2,2 !}\left(F \otimes \mathbf{k}_{M}\right), \mathrm{R} p_{1,1,2 *} p_{2}^{!} G\right) \\
& \simeq \operatorname{Hom}\left(F \otimes \mathbf{k}_{M}, p_{1,2,2} \mathrm{R} p_{1,1,2 *} p_{2}^{!} G\right) \\
& \simeq \operatorname{Hom}\left(F \otimes \mathbf{k}_{M}, \mathrm{R} \delta_{*} \delta^{!} p_{2}^{!} G\right) \\
& \simeq \operatorname{Hom}\left(\delta^{-1}\left(F \otimes \mathbf{k}_{M}\right), \delta^{!} p_{2}^{!} G\right) \\
& \simeq \operatorname{Hom}(F, G) .
\end{aligned}
$$

Q.E.D.

Similarly, we have the following D-module version. Here again, we write for short Hom instead of $\operatorname{Hom}_{\mathscr{D}_{Y}}$ with $Y=X, X \times X \times X$.

Lemma 7.7.6. Let $X$ be a complex manifold and let $\mathscr{M}, \mathscr{N} \in \mathrm{D}_{\mathrm{hol}}^{\mathrm{b}}\left(\mathscr{D}_{X}\right)$. Then we have the isomorphisms

$$
\begin{aligned}
& \operatorname{Hom}\left(\mathscr{M} \stackrel{\mathrm{D}}{\otimes} \mathscr{B}_{\Delta_{X}}\left[-d_{X}\right], \mathscr{B}_{\Delta_{X}}\left[d_{X}\right] \stackrel{\mathrm{D}}{\otimes} \mathscr{N}\right) \simeq \operatorname{Hom}(\mathscr{M}, \mathscr{N}), \\
& \operatorname{Hom}\left(\mathscr{B}_{\Delta_{M}}\left[-d_{X}\right] \stackrel{\mathrm{D}}{\otimes} \mathscr{M}, \mathscr{N} \stackrel{\mathrm{D}}{\otimes} \mathscr{B}_{\Delta_{X}}\left[d_{X}\right]\right) \simeq \operatorname{Hom}(\mathscr{M}, \mathscr{N}) .
\end{aligned}
$$


As applications of Theorem 7.7.4, we obtain the following corollaries.

Proposition 7.7.7. Let $f: X \rightarrow Y$ be a morphism of complex manifolds. Then, for any $\mathscr{N} \in \mathrm{D}_{\text {hol }}^{\mathrm{b}}\left(\mathscr{D}_{Y}\right)$,

$$
\mathcal{S}_{X}^{\mathrm{E}}\left(\mathrm{D} f^{*} \mathscr{N}\right) \simeq \mathrm{E} f^{-1} \mathcal{S} o l_{Y}^{\mathrm{E}}(\mathscr{N}) .
$$

Proof. We have

$$
\begin{aligned}
\mathcal{S o l}_{X}^{\mathrm{E}}\left(\mathrm{D}^{*} \mathscr{N}\right) & \simeq \mathrm{D}_{X}^{\mathrm{E}} \mathcal{D} \mathcal{R}_{X}^{\mathrm{E}}\left(\mathrm{D}^{*} \mathscr{N}\right)\left[-d_{X}\right] \\
& \simeq \mathrm{D}_{X}^{\mathrm{E}} \mathrm{E} f^{!} \mathcal{D} \mathcal{R}_{Y}^{\mathrm{E}}(\mathscr{N})\left[-d_{Y}\right] \\
& \simeq \mathrm{E} f^{-1} \mathrm{D}_{X}^{\mathrm{E}} \mathcal{D} \mathcal{R}_{Y}^{\mathrm{E}}(\mathscr{N})\left[-d_{Y}\right] \\
& \simeq \mathrm{E} f^{-1} \mathcal{S}_{l_{Y}}^{\mathrm{E}}(\mathscr{N}) .
\end{aligned}
$$

Q.E.D.

Corollary 7.7.8. Let $X$ be a complex manifold and $\mathscr{M}, \mathscr{N} \in \mathrm{D}_{\text {hol }}^{\mathrm{b}}\left(\mathscr{D}_{X}\right)$. Then we have the isomorphisms

$$
\begin{aligned}
& \mathcal{D} \mathcal{R}_{X}^{\mathrm{E}}(\mathscr{M} \stackrel{\mathrm{D}}{\otimes} \mathscr{N}) \simeq \mathrm{E} \delta^{!}\left(\mathcal{D} \mathcal{R}_{X}^{\mathrm{E}}(\mathscr{M}) \stackrel{+}{\otimes} \mathcal{D} \mathcal{R}_{X}^{\mathrm{E}}(\mathscr{N})\right)\left[d_{X}\right], \\
& \mathcal{S}_{\text {ol }}^{\mathrm{E}}(\mathscr{M} \stackrel{\mathrm{D}}{\otimes} \mathscr{N}) \simeq \mathcal{S}_{X}^{\mathrm{E}}(\mathscr{M}) \stackrel{+}{\otimes} \mathcal{S}_{X}^{\mathrm{E}}(\mathscr{N}),
\end{aligned}
$$

where $\delta: X \rightarrow X \times X$ is the diagonal embedding.

Proof. Since $\mathscr{M} \stackrel{\mathrm{D}}{\otimes} \mathscr{N} \simeq \mathrm{D} \delta^{*}(\mathscr{M} \stackrel{\mathrm{D}}{\otimes} \mathscr{N})$, it is enough to apply (7.3.2) and (7.7.12).

Q.E.D.

Corollary 7.7.9. For a closed hypersurface $Y$ of a complex manifold $X$ and $\mathscr{M} \in \mathrm{D}_{\text {hol }}^{\mathrm{b}}\left(\mathscr{O}_{X}\right)$, we have

$$
\operatorname{Sol}_{X}^{\mathrm{E}}(\mathscr{M}(* Y)) \simeq \pi^{-1} \mathbb{C}_{X \backslash Y} \otimes \mathcal{S}_{\text {ol }}^{\mathrm{E}}(\mathscr{M}) .
$$

Proof. It follows from Theorem 7.7.4 and isomorphisms

$$
\mathcal{D} \mathcal{R}_{X}^{\mathrm{E}}(\mathscr{M}(* Y)) \simeq \operatorname{R} \mathscr{I h o m}\left(\pi^{-1} \mathbb{C}_{X \backslash Y}, \mathcal{D} \mathcal{R}_{X}^{\mathrm{E}}(\mathscr{M})\right)
$$

and

$$
\mathrm{D}_{X}^{\mathrm{E}}\left(\mathrm{R} \mathscr{I h o m}\left(\pi^{-1} \mathbb{C}_{X \backslash Y}, \mathcal{D} \mathcal{R}_{X}^{\mathrm{E}}(\mathscr{M})\right) \simeq \pi^{-1} \mathbb{C}_{X \backslash Y} \otimes \mathrm{D}_{X}^{\mathrm{E}} \mathcal{D} \mathcal{R}_{X}^{\mathrm{E}}(\mathscr{M})\right.
$$

(see Theorem 6.6.3 (v)).

Q.E.D. 
Corollary 7.7.10. For a closed hypersurface $Y$ of a complex manifold $X$ and $\varphi \in \mathscr{O}_{X}(* Y)$, we have

$$
\operatorname{Sol}_{X}^{\mathrm{E}}\left(\mathscr{E}_{X \backslash Y \mid X}^{\varphi}\right) \simeq \mathbb{C}_{X}^{\mathrm{E}} \stackrel{+}{\otimes} \mathbb{C}_{\{t=-\operatorname{Re} \varphi\}} .
$$

This follows from Proposition 7.3.1, (6.5.2) and Theorem 7.7.4, because one has $\mathscr{E}_{X \backslash Y \mid X}^{\varphi} \simeq\left(\mathbb{D}_{X} \mathscr{E}_{X \backslash Y \mid X}^{-\varphi}\right)(* Y)$.

\subsection{Enhanced Riemann-Hilbert correspondence}

The following theorem is the main theorem.

Theorem 7.8.1 (Generalized Riemann-Hilbert correspondence). There exists a canonical isomorphism functorial with respect to $\mathscr{M} \in \mathrm{D}_{\text {hol }}^{\mathrm{b}}\left(\mathscr{D}_{X}\right)$ :

$$
\mathscr{M} \stackrel{\mathrm{D}}{\otimes} \mathscr{O}_{X}^{\mathrm{E}} \stackrel{\sim}{\longrightarrow} \mathscr{I h o m}^{+}\left(\mathcal{S}_{\text {ol }}^{\mathrm{E}}(\mathscr{M}), \mathscr{O}_{X}^{\mathrm{E}}\right) \text { in } \mathrm{E}^{\mathrm{b}}\left(\mathrm{I}_{X}\right) \text {. }
$$

The proof is parallel with the one of Theorem 4.3 .2 by reducing the problem to the case where $\mathscr{M}$ is an exponential D-module. However, in this case, we can treat $\mathcal{D} \mathcal{R}_{X}^{\mathrm{E}}(\mathscr{M})$ by Proposition 7.3 .1 , but not $\mathcal{S}_{\text {ol }} l_{X}^{\mathrm{E}}(\mathscr{M})$. In order to calculate it, we need the commutativity of the enhanced de Rham functor and the duality functor (see Theorem 7.7.4 and its consequence Corollary 7.7.9).

Sketch of the proof of Theorem 7.8.1. First we shall construct a morphism (7.8.1). We have a canonical morphism

$$
\mathscr{O}_{X}^{\mathrm{E}} \stackrel{+}{\otimes}_{\beta \mathscr{O}_{X}} \mathscr{O}_{X}^{\mathrm{E}} \longrightarrow \mathscr{O}_{X}^{\mathrm{E}}
$$

Hence we have

$$
\left(\mathscr{M}^{\mathrm{D}} \otimes \mathscr{O}_{X}^{\mathrm{E}}\right)^{+} \mathcal{S} l_{X}^{\mathrm{E}}(\mathscr{M}) \longrightarrow \mathscr{O}_{X}^{\mathrm{E}} \stackrel{+}{\otimes}_{\beta \mathscr{O}_{X}} \mathscr{O}_{X}^{\mathrm{E}} \longrightarrow \mathscr{O}_{X}^{\mathrm{E}}
$$

which induces a morphism

$$
\mathscr{M} \stackrel{\mathrm{D}}{\otimes} \mathscr{O}_{X}^{\mathrm{E}} \longrightarrow \mathscr{I h o m}^{+}\left(\mathcal{S}_{0} l_{X}^{\mathrm{E}}(\mathscr{M}), \mathscr{O}_{X}^{\mathrm{E}}\right) .
$$

In order to see that it is an isomorphism, we shall apply Lemma 7.5.5, where $P_{X}(\mathscr{M})$ is the statement that (7.8.2) is an isomorphism. 
We shall only check property (f) of this lemma. Hence, we assume that $\mathscr{M}$ has a normal form along a normal crossing divisor $D$. Then we have $\mathcal{S}^{\mathrm{E}} l_{X}^{\mathrm{E}}(\mathscr{M}) \simeq \pi^{-1} \mathbb{C}_{X \backslash D} \otimes \mathcal{S}_{\text {ol }} \mathrm{E}(\mathscr{M})$ by Corollary 7.7.9, which implies that

$$
\mathscr{I h o m}^{+}\left(\mathcal{S}_{\mathrm{al}}^{\mathrm{E}}(\mathscr{M}), \mathscr{O}_{X}^{\mathrm{E}}\right) \simeq \mathscr{I h o m}^{+}\left(\operatorname{Sol}_{X}^{\mathrm{E}}(\mathscr{M}), \mathscr{O}_{X}^{\mathrm{E}}(* D)\right) \text {. }
$$

Let $\varpi: \widetilde{X} \rightarrow X$ be the real blow up of $X$ along $D$. Then we have

$$
\mathscr{M} \stackrel{\mathrm{D}}{\otimes} \mathscr{O}_{X}^{\mathrm{E}} \simeq \mathrm{E}_{*}\left(\mathscr{M}^{\mathcal{A}} \stackrel{\mathrm{L}}{\otimes}_{\mathcal{A}_{\tilde{X}}} \mathscr{O}_{\tilde{X}}^{\mathrm{E}}\right)
$$

and

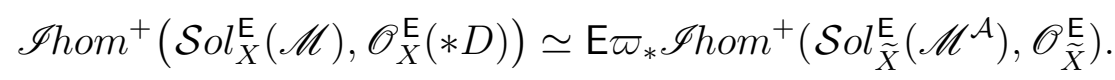

Hence it is enough to show that

$$
\mathscr{M}^{\mathcal{A}} \stackrel{\mathrm{L}}{\otimes}_{\mathcal{A}_{\tilde{X}}} \mathscr{O}_{\tilde{X}}^{\mathrm{E}} \longrightarrow \mathscr{I h o m}^{+}\left(\operatorname{Sol}_{\widetilde{X}}^{\mathrm{E}}\left(\mathscr{M}^{\mathcal{A}}\right), \mathscr{O} \mathrm{E}_{\tilde{X}}^{\mathrm{E}}\right)
$$

is an isomorphism.

Since the question is local and $\mathscr{M}^{\mathcal{A}}$ is locally isomorphic to a direct sum of exponential D-modules $\left(\mathscr{E}_{X \backslash D \mid X}^{\varphi}\right)^{\mathcal{A}}$ with $\varphi \in \mathscr{O}_{X}(* D)$, we may assume that $\mathscr{M}^{\mathcal{A}}=\left(\mathscr{E}_{X \backslash D \mid X}^{\varphi}\right)^{\mathcal{A}}$. Since (7.8.3) is the image of (7.8.2) by the functor $\mathrm{E} \varpi$ !, it is enough to show that (7.8.2) is an isomorphism when $\mathscr{M}=\mathscr{E}_{X \backslash D \mid X}$.

In this case, Corollary 7.7.10 implies that

$$
\mathcal{S o l}_{X}^{\mathrm{E}}(\mathscr{M}) \simeq \mathbb{C}_{X}^{\mathrm{E}} \stackrel{+}{\otimes} \mathbb{C}_{\{t=-\operatorname{Re} \varphi\}},
$$

and we can easily see that (7.8.2) is an isomorphism.

Q.E.D.

Corollary 7.8.2. There exists a canonical isomorphism functorial with respect to $\mathscr{M} \in \mathrm{D}_{\mathrm{hol}}^{\mathrm{b}}\left(\mathscr{D}_{X}\right)$ :

$$
\mathscr{M} \stackrel{\mathrm{D}}{\otimes} \mathscr{O}_{X}^{\mathrm{t}} \stackrel{\sim}{\longrightarrow} \mathscr{I h o m}^{\mathrm{E}}\left(\mathcal{S}_{\text {ol }}^{\mathrm{E}}(\mathscr{M}), \mathscr{O}_{X}^{\mathrm{E}}\right) \text { in } \mathrm{D}^{\mathrm{b}}\left(\mathrm{I}_{X}\right) .
$$

Proof. Let us apply the functor $\mathscr{I} \operatorname{hom}^{\mathrm{E}}\left(\mathbb{C}_{X}^{\mathrm{E}}, \bullet\right)$ to the isomorphism (7.8.1). Since $\mathscr{I h o m}{ }^{\mathrm{E}}\left(\mathbb{C}_{X}^{\mathrm{E}}, \mathscr{O}_{X}^{\mathrm{E}}\right) \simeq \mathscr{O}_{X}^{\mathrm{t}}$ by $(7.2 .3)$, we get

$$
\mathscr{I h o m}{ }^{\mathrm{E}}\left(\mathbb{C}_{X}^{\mathrm{E}}, \mathscr{M} \stackrel{\mathrm{D}}{\otimes} \mathscr{O}_{X}^{\mathrm{E}}\right) \simeq \mathscr{M} \stackrel{\mathrm{D}}{\otimes} \mathscr{O}_{X}^{\mathrm{t}} .
$$


On the other-hand, we have

$$
\begin{aligned}
\mathscr{I h o m}^{\mathrm{E}}\left(\mathbb{C}_{X}^{\mathrm{E}}, \mathscr{I h o m}^{+}\left(\mathcal{S}_{\operatorname{lol}}^{\mathrm{E}}(\mathscr{M}), \mathscr{O}_{X}^{\mathrm{E}}\right)\right) \\
\simeq \mathscr{I h o m}^{\mathrm{E}}\left(\mathcal{S}_{X}^{\mathrm{E}}(\mathscr{M}), \mathscr{I h o m}^{+}\left(\mathbb{C}_{X}^{\mathrm{E}}, \mathscr{O}_{X}^{\mathrm{E}}\right)\right) \\
\simeq \mathscr{I h o m}^{\mathrm{E}}\left(\mathcal{S}^{\mathrm{E}} l_{X}^{\mathrm{E}}(\mathscr{M}), \mathscr{O}_{X}^{\mathrm{E}}\right) .
\end{aligned}
$$

Q.E.D.

Corollary 7.8.3 (Enhanced Riemann-Hilbert correspondence). There exists a canonical isomorphism functorial with respect to $\mathscr{M} \in \mathrm{D}_{\text {hol }}^{\mathrm{b}}\left(\mathscr{D}_{X}\right)$ :

$$
\mathscr{M} \stackrel{\sim}{\longrightarrow} \mathscr{H}_{0}{ }^{\mathrm{E}}\left(\mathcal{S}_{X}^{\mathrm{E}}(\mathscr{M}), \mathscr{O}_{X}^{\mathrm{E}}\right) \text { in } \mathrm{D}^{\mathrm{b}}\left(\mathscr{D}_{X}\right) .
$$

Proof. Apply the functor $\alpha_{X}$ to (7.8.4).

Q.E.D.

By Corollary 7.8.3, we can show the following full faithfulness of the enhanced de Rham functor.

Theorem 7.8.4. For $\mathscr{M}, \mathscr{N} \in \mathrm{D}_{\text {hol }}^{\mathrm{b}}\left(\mathscr{D}_{X}\right)$, one has an isomorphism

$$
\mathrm{R}^{H}{ }_{0 m}{ }_{\mathscr{D}_{X}}(\mathscr{M}, \mathscr{N}) \stackrel{\sim}{\longrightarrow} \mathscr{H}_{o m}^{\mathrm{E}}\left(\mathcal{D} \mathcal{R}_{X}^{\mathrm{E}} \mathscr{M}, \mathcal{D} \mathcal{R}_{X}^{\mathrm{E}} \mathscr{N}\right) .
$$

In particular, the functor

$$
\mathcal{D} \mathcal{R}_{X}^{\mathrm{E}}: \mathrm{D}_{\mathrm{hol}}^{\mathrm{b}}\left(\mathscr{D}_{X}\right) \longrightarrow \mathrm{E}_{\mathbb{R}-c}^{\mathrm{b}}\left(\mathrm{IC}_{X}\right)
$$

is fully faithful.

Proof. By Theorem 7.7.4 and Theorem 6.6.3 (vi), we have

$$
\mathscr{H} o m^{\mathrm{E}}\left(\mathcal{D} \mathcal{R}_{X}^{\mathrm{E}} \mathscr{M}, \mathcal{D} \mathcal{R}_{X}^{\mathrm{E}} \mathscr{N}\right) \simeq \mathscr{H} o m^{\mathrm{E}}\left(\mathcal{S}_{\mathrm{X}}^{\mathrm{E}}{ }_{X}^{\mathrm{N}}, \mathcal{S}_{\mathrm{X}}^{\mathrm{E}} \mathscr{M}\right)
$$

Now, we have

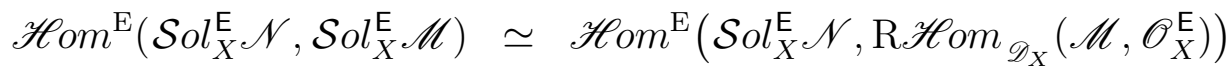

$$
\begin{aligned}
& \simeq \operatorname{R} \mathscr{H}_{o m} \mathscr{D}_{X}\left(\mathscr{M}, \mathscr{H} o m^{\mathrm{E}}\left(\mathcal{S}_{o l}^{\mathrm{E}} \mathscr{N}, \mathscr{O}_{X}^{\mathrm{E}}\right)\right) \\
& \simeq \mathrm{R} \mathscr{H}_{o m_{X}}(\mathscr{M}, \mathscr{N}) \text {. }
\end{aligned}
$$

Here the last isomorphism follows from Corollary 7.8.3.

Q.E.D.

Remark 7.8.5. Corollary 7.8.3 and Theorem 7.8.4 due to [DK 13, Th. 9.6.1, Th. 9.7.1] are a natural formulation of the Riemann-Hilbert correspondence for irregular D-modules. Theorem 7.8.1 due to [KS14, Th. 4.5] is a generalization to the irregular case of Theorem 4.3 .2 which is itself a generalization/reformulation of a theorem of J-E. Björk ([Bj 93]). 


\section{Integral transforms}

\subsection{Integral transforms with irregular kernels}

Theorem 8.1.1. Let $X$ be a complex manifold and let $\mathscr{L} \in \mathrm{D}_{\mathrm{hol}}^{\mathrm{b}}\left(\mathscr{D}_{X}\right)$ and $\mathscr{M} \in \mathrm{D}^{\mathrm{b}}\left(\mathscr{D}_{X}\right)$. There is a natural isomorphism

$$
\mathcal{D R}_{X}^{\mathrm{E}}(\mathscr{L} \stackrel{\mathrm{D}}{\otimes} \mathscr{M}) \simeq \mathscr{I h o m}{ }^{+}\left(\mathcal{S}_{\mathrm{l}}^{\mathrm{E}}(\mathscr{L}), \mathcal{D} \mathcal{R}_{X}^{\mathrm{E}}(\mathscr{M})\right) .
$$

Proof. By Theorem 7.8.1, we have an isomorphism in $\mathrm{E}^{\mathrm{b}}\left(\mathrm{I} \mathscr{D}_{X}\right)$ :

$$
\mathscr{L} \stackrel{\mathrm{D}}{\otimes} \mathscr{O}_{X}^{\mathrm{E}} \sim \mathscr{I h o m}^{+}\left(\mathcal{S}^{\mathrm{E}} l_{X}^{\mathrm{E}}(\mathscr{L}), \mathscr{O}_{X}^{\mathrm{E}}\right) .
$$

Let us apply $\mathscr{M}^{\mathrm{r}} \stackrel{\mathrm{L}}{\otimes}_{\mathscr{D}_{X}} \cdot$ to both sides of (8.1.1). We have

$$
\begin{aligned}
\mathscr{M}^{\mathrm{r}} \stackrel{\mathrm{L}}{\otimes}_{\mathscr{D}_{X}}\left(\mathscr{L} \stackrel{\mathrm{D}}{\otimes} \mathscr{O}_{X}^{\mathrm{E}}\right) & \simeq(\mathscr{M} \stackrel{\mathrm{D}}{\otimes} \mathscr{L})^{\mathrm{r}} \stackrel{\mathrm{L}}{\otimes} \mathscr{D}_{X} \mathscr{O}_{X}^{\mathrm{E}} \\
& \simeq \mathcal{D} \mathcal{R}_{X}^{\mathrm{E}}(\mathscr{M} \stackrel{\mathrm{D}}{\otimes} \mathscr{L}),
\end{aligned}
$$

and

$$
\begin{aligned}
\mathscr{M}^{\mathrm{r}} \stackrel{\mathrm{L}}{\otimes}_{\mathscr{D} X} \mathscr{I h o m}^{+}\left(\mathcal{S o l}_{X}^{\mathrm{E}}(\mathscr{L}), \mathscr{O}_{X}^{\mathrm{E}}\right) & \underset{(\mathrm{a})}{\simeq} \mathscr{I h o m}^{+}\left(\mathcal{S o l}_{X}^{\mathrm{E}}(\mathscr{L}), \mathscr{M}^{\mathrm{r}}{\stackrel{\mathrm{Q}}{\mathscr{D}_{X}}} \mathscr{O}_{X}^{\mathrm{E}}\right) \\
& \simeq \mathscr{I h o m}^{+}\left(\mathcal{S o l}_{X}^{\mathrm{E}}(\mathscr{L}), \mathcal{D} \mathcal{R}_{X}^{\mathrm{E}}(\mathscr{M})\right) .
\end{aligned}
$$

(We do not give the proof of isomorphism (a) and refer to [KS 14, Lem. 3.12].) Q.E.D.

Consider morphisms of complex manifolds

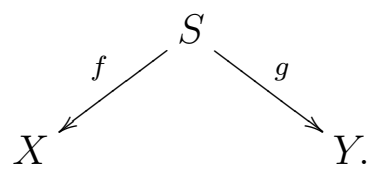

Notation 8.1.2. (i) For $\mathscr{M} \in \mathrm{D}_{\text {q-good }}^{\mathrm{b}}\left(\mathscr{D}_{X}\right)$ and $\mathscr{L} \in \mathrm{D}_{\text {q-good }}^{\mathrm{b}}\left(\mathscr{D}_{S}\right)$ recall that one sets

$$
\mathscr{M} \stackrel{\mathrm{D}}{\circ} \mathscr{L}:=\mathrm{D} g_{*}\left(\mathrm{D} f^{*} \mathscr{M} \stackrel{\mathrm{D}}{\otimes} \mathscr{L}\right)
$$


(ii) For $L \in \mathrm{E}^{\mathrm{b}}\left(\mathrm{IC}_{S}\right), F \in \mathrm{E}^{\mathrm{b}}\left(\mathrm{IC}_{X}\right)$ and $G \in \mathrm{E}^{\mathrm{b}}\left(\mathrm{IC}_{Y}\right)$ one sets

$$
\begin{aligned}
& L \stackrel{\mathrm{E}}{\circ} G:=\mathrm{E} f_{! !}\left(L \stackrel{+}{\otimes} \mathrm{E} g^{-1} G\right), \\
& \Phi_{L}^{\mathrm{E}}(G)=L \stackrel{\mathrm{E}}{\circ} G, \quad \Psi_{L}^{\mathrm{E}}(F)=\mathrm{E} g_{*} \mathscr{I} h o m^{+}\left(L, \mathrm{E} f^{!} F\right) .
\end{aligned}
$$

Note that we have a pair of adjoint functors

$$
\Phi_{L}^{\mathrm{E}}: \mathrm{E}^{\mathrm{b}}\left(\mathrm{I}_{Y}\right) \rightleftarrows \mathrm{E}^{\mathrm{b}}\left(\mathrm{I}_{X}\right): \Psi_{L}^{\mathrm{E}}
$$

Theorem 8.1.3. Let $\mathscr{M} \in \mathrm{D}_{\text {q-good }}^{\mathrm{b}}\left(\mathscr{D}_{X}\right), \mathscr{L} \in \mathrm{D}_{\text {g-hol }}^{\mathrm{b}}\left(\mathscr{D}_{S}\right):=\mathrm{D}_{\text {hol }}^{\mathrm{b}}\left(\mathscr{D}_{S}\right) \cap$ $\mathrm{D}_{\text {good }}^{\mathrm{b}}\left(\mathscr{D}_{S}\right)$ and let $L:=\operatorname{Sol}_{S}^{\mathrm{E}}(\mathscr{L})$. Assume that $f^{-1} \operatorname{Supp}(\mathscr{M}) \cap \operatorname{Supp}(\mathscr{L})$ is proper over $Y$. Then there is a natural isomorphism in $\mathrm{E}^{\mathrm{b}}\left(\mathrm{IC}_{Y}\right)$ :

$$
\Psi_{L}^{\mathrm{E}}\left(\mathcal{D} \mathcal{R}_{X}^{\mathrm{E}}(\mathscr{M})\right)\left[d_{X}-d_{S}\right] \simeq \mathcal{D} \mathcal{R}_{Y}^{\mathrm{E}}(\mathscr{M} \stackrel{\mathrm{D}}{\circ} \mathscr{L}) .
$$

Proof. The proof goes as in the regular case (Theorem 4.4.2) by using Theorems 7.3.2 and 8.1.1.

Q.E.D.

Corollary 8.1.4. In the situation of Theorem 8.1.3, let $G \in \mathrm{E}^{\mathrm{b}}\left(\mathrm{I}_{Y}\right)$. Then there is a natural isomorphism in $\mathrm{D}^{\mathrm{b}}(\mathbb{C})$

$$
\begin{aligned}
& \operatorname{RHom}^{\mathrm{E}}\left(L \stackrel{\mathrm{E}}{\circ} G, \Omega_{X}^{\mathrm{E}} \stackrel{\mathrm{L}}{\otimes}_{\mathscr{D}_{X}} \mathscr{M}\right)\left[d_{X}-d_{S}\right] \\
& \simeq \operatorname{RHom}^{\mathrm{E}}\left(G, \Omega_{Y}^{\mathrm{E}} \stackrel{\mathrm{L}}{\otimes_{\mathscr{D}_{Y}}}(\mathscr{M} \stackrel{\mathrm{D}}{\circ} \mathscr{L})\right) .
\end{aligned}
$$

Proof. This follows from Theorem 8.1.3 and the adjunction (8.1.3). Q.E.D.

Note that Corollary 8.1.4 is a generalization of [KS01, Th.7.4.12] to not necessarily regular holonomic D-modules.

\subsection{Enhanced Fourier-Sato transform}

The results in $\S 6$ extend to the case where $M$ is replaced with a bordered space $M_{\infty}$. Thus $\pi$ denotes the projection $M_{\infty} \times \mathbb{R}_{\infty} \rightarrow M_{\infty}$ and $t$ the coordinate of $\mathbb{R}$. One defines $\mathrm{E}^{\mathrm{b}}\left(\mathrm{Ik}_{M_{\infty}}\right)$ as the quotient triangulated category $\mathrm{D}^{\mathrm{b}}\left(\mathrm{Ik}_{M_{\infty} \times \mathbb{R}_{\infty}}\right) /\left\{K ; \pi^{-1} \mathrm{R} \pi_{*} K \stackrel{\sim}{\sim} K\right\}$.

One defines the functors

$$
\begin{aligned}
e_{M_{\infty}}, \epsilon_{M_{\infty}}: & \mathrm{D}^{\mathrm{b}}\left(\mathrm{Ik}_{M_{\infty}}\right) \rightarrow \mathrm{E}^{\mathrm{b}}\left(\mathrm{I}_{M_{\infty}}\right), \\
& e_{M_{\infty}}(F)=\mathbf{k}_{M_{\infty}}^{\mathrm{E}} \otimes \pi^{-1} F, \quad \epsilon_{M_{\infty}}(F)=\mathbf{k}_{\{t \geq 0\}} \otimes \pi^{-1} F .
\end{aligned}
$$


Note that $e_{M_{\infty}}(F) \simeq \mathbf{k}_{M_{\infty}}^{\mathrm{E}} \stackrel{+}{\otimes} \epsilon_{M_{\infty}}(F)$.

Then Proposition 6.5.9 extends to bordered spaces.

Proposition 8.2.1. The functors $e_{M_{\infty}}$ and $\epsilon_{M_{\infty}}$ are fully faithful.

Let $\mathrm{V}$ be a finite-dimensional real vector space, $\mathrm{V}^{*}$ its dual. Recall that the Fourier-Sato transform is an equivalence of categories between conic sheaves on $\mathrm{V}$ and conic sheaves on $\mathrm{V}^{*}$. References are made to [KS90]. In [Ta 08], D. Tamarkin has extended the Fourier-Sato transform to no more conic (usual) sheaves, by adding an extra variable. Here we generalize this last transform to enhanced ind-sheaves on the bordered space $\mathrm{V}_{\infty}$.

We set $n=\operatorname{dim} \mathrm{V}$ and we denote by or $\mathrm{V}$ the orientation $\mathrm{k}$-module of $\mathrm{V}$, i.e., or $\mathrm{v}=H_{c}^{n}\left(\mathrm{~V} ; \mathrm{k}_{\mathrm{V}}\right)$. In this subsection, the base field $\mathbf{k}$ is an arbitrary field. We have a canonical isomorphism or $\vee \simeq$ or $_{\mathrm{V}^{*}}$. We denote by $\Delta_{\mathrm{V}}$ the diagonal of $\mathrm{V} \times \mathrm{V}$.

We consider the bordered space $\mathrm{V}_{\infty}=(\mathrm{V}, \overline{\mathrm{V}})$ where $\overline{\mathrm{V}}$ is the projective compactification of $\mathrm{V}$, that is

$$
\overline{\mathrm{V}}=((\mathrm{V} \oplus \mathbb{R}) \backslash\{0\}) / \mathbb{R}^{\times}
$$

We introduce the kernels

$$
\begin{gathered}
L_{\mathrm{V}}:=\mathbf{k}_{\{t=\langle x, y\rangle\}} \in \mathrm{E}^{\mathrm{b}}\left(\mathrm{I}_{\mathrm{V}_{\infty} \times \mathrm{V}_{\infty}^{*}}\right), \\
L_{\mathrm{V}}^{a}:=\mathbf{k}_{\{t=-\langle x, y\rangle\}} \in \mathrm{E}^{\mathrm{b}}\left(\mathrm{I}_{\mathrm{V}_{\infty} \times \mathrm{V}_{\infty}^{*}}\right), \\
L_{\mathrm{V}^{*}}^{a}:=\mathbf{k}_{\{t=-\langle x, y\rangle\}} \in \mathrm{E}^{\mathrm{b}}\left(\mathrm{I}_{\mathrm{V}_{\infty}^{*} \times \mathrm{V}_{\infty}}\right) .
\end{gathered}
$$

Here, $x$ and $y$ denote points of $\mathrm{V}$ and $\mathrm{V}^{*}$, respectively.

Lemma 8.2.2. One has isomorphisms in $\mathrm{E}^{\mathrm{b}}\left(\mathrm{I}_{\mathrm{V}_{\infty} \times \mathrm{v}_{\infty}}\right)$

$$
\begin{aligned}
& L_{\mathrm{V}} \stackrel{\mathrm{E}}{\circ} L_{\mathrm{V}^{*}}^{a} \stackrel{\sim}{\sim} \mathbf{k}_{\Delta_{\mathrm{V}} \times\{t=0\}} \otimes \text { or } \mathrm{V}[-n], \\
& L_{\mathrm{V}^{*}}^{a} \stackrel{\stackrel{\mathrm{E}}{\circ}}{L_{\mathrm{V}}} \stackrel{\sim}{\longrightarrow} \mathbf{k}_{\Delta_{\mathrm{V}^{*}} \times\{t=0\}} \otimes \text { or } \mathrm{V}[-n] .
\end{aligned}
$$

Now we introduce the enhanced Fourier-Sato functors

$$
\begin{array}{cc}
\mathrm{E}_{\mathscr{F}}: \mathrm{E}^{\mathrm{b}}\left(\mathrm{I}_{\mathrm{V}_{\infty}}\right) \rightarrow \mathrm{E}^{\mathrm{b}}\left(\mathrm{I}_{\mathrm{V}_{\infty}^{*}}\right), & \mathrm{E}_{\mathscr{F}}(F)=F \stackrel{\mathrm{E}}{\circ} L_{\mathrm{V}}, \\
\mathrm{E}_{\mathrm{F}^{*}}^{a}: \mathrm{E}^{\mathrm{b}}\left(\mathrm{Ik}_{\mathrm{V}_{\infty}^{*}}\right) \rightarrow \mathrm{E}^{\mathrm{b}}\left(\mathrm{I}_{\mathrm{V}_{\infty}}\right), \quad \mathscr{E}_{\mathrm{V}^{*}}^{a}(F)=F \stackrel{\mathrm{E}}{\circ} L_{\mathrm{V}^{*}}^{a} .
\end{array}
$$

Applying Lemma 8.2.2, we obtain: 
Theorem 8.2.3 (See [Ta08]). The functors $\mathrm{E}_{\mathscr{F}}$ and $\mathrm{E}_{\mathscr{F}_{\mathrm{V}^{*}}^{a}}^{a} \otimes$ or $\mathrm{V}[n]$ are equivalences of categories, inverse to each other. In other words, one has the isomorphisms, functorial with respect to $F \in \mathrm{E}^{\mathrm{b}}\left(\mathrm{I}_{\mathrm{V}_{\infty}}\right)$ and $G \in \mathrm{E}^{\mathrm{b}}\left(\mathrm{Ik}_{\mathrm{V}_{\infty}^{*}}\right)$ :

$$
\begin{aligned}
& \mathrm{E}_{\mathscr{F}_{\mathrm{V}^{*}}^{a}}^{a} \mathrm{E}_{\mathscr{F}}(F) \simeq F \otimes \text { or }_{\mathrm{V}}[-n], \\
& \mathrm{E}_{F_{\mathrm{V}}} \circ \mathrm{E}_{\mathscr{F}_{\mathrm{V}^{*}}}^{a}(G) \simeq G \otimes \operatorname{or}_{\mathrm{V}}[-n] .
\end{aligned}
$$

Corollary 8.2.4. The two functors $\mathrm{E}_{\mathrm{V}}(\cdot)$ and $\Psi_{L_{\mathrm{V}}^{a}}^{\mathrm{E}}(\cdot) \otimes \operatorname{or}_{\mathrm{V}}[-n]$ are isomorphic.

Corollary 8.2.5. There is an isomorphism functorial in $F_{1}, F_{2} \in \mathrm{E}^{\mathrm{b}}\left(\mathrm{Ik}_{\vee_{\infty}}\right)$ :

$$
\operatorname{RHom}^{\mathrm{E}}\left(F_{1}, F_{2}\right) \simeq \operatorname{RHom}^{\mathrm{E}}\left({ }^{\mathrm{E}} \mathscr{F}_{\mathrm{V}}\left(F_{1}\right),{ }^{\mathrm{E}} \mathscr{F}_{\mathrm{V}}\left(F_{2}\right)\right) \text {. }
$$

Recall that one denotes by $D_{\mathbb{R}^{+}}^{\mathrm{b}}\left(\mathbf{k}_{\mathrm{V}}\right)$ the full subcategory of $\mathrm{D}^{\mathrm{b}}\left(\mathrm{k}_{\mathrm{V}}\right)$ consisting of conic sheaves (see [KS90]). Here conic sheaves mean sheaves on $\mathrm{V}$ constant on any half line $\mathbb{R}_{>0} v(v \in \mathrm{V} \backslash\{0\})$. We shall denote here by $\mathrm{S}_{\mathscr{F}}(F)$ the Fourier-Sato transform of $F \in \mathrm{D}_{\mathbb{R}^{+}}^{\mathrm{b}}\left(\mathbf{k}_{\mathrm{V}}\right)$, which was denoted by $F^{\wedge}$ in loc. cit. The functor ${ }^{S} \mathscr{F}_{\mathfrak{V}}: D_{\mathbb{R}^{+}}^{b}\left(\mathbf{k}_{\mathrm{V}}\right) \rightarrow D_{\mathbb{R}^{+}}^{\mathrm{b}}\left(\mathbf{k}_{\mathrm{V}^{*}}\right)$ is an equivalence of categories.

Recall that one identifies the sheaf $\mathbf{k}_{\{t \geq 0\}}$ with its image in $\mathrm{E}^{\mathrm{b}}\left(\mathrm{I}_{\mathrm{V} \times \mathbb{R}_{\infty}}\right)$ and that the functor

$$
\epsilon_{\mathrm{V}_{\infty}}: \mathrm{D}^{\mathrm{b}}\left(\mathbf{k}_{\mathrm{V}}\right) \hookrightarrow \mathrm{E}^{\mathrm{b}}\left(\mathrm{I}_{\mathrm{V}_{\infty}}\right), \quad \epsilon_{\mathrm{V}_{\infty}}(F)=\mathbf{k}_{\{t \geq 0\}} \otimes \pi^{-1} F
$$

is a fully faithful embedding by Proposition 8.2.1.

Consider the diagram of categories and functors

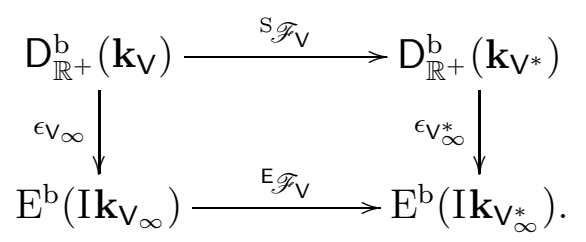

Theorem 8.2.6. Diagram (8.2.6) is quasi-commutative.

\subsection{Laplace transform}

In the sequel, we take $\mathbb{C}$ as the base field $\mathbf{k}$. Recall the $\mathscr{D}_{X}$-module $\mathscr{E}_{U \mid X}^{\varphi}$ and Notation 7.1.1. We saw in Proposition 7.1.2 that

$$
\operatorname{Sol}_{X}^{\mathrm{E}}\left(\mathscr{E}_{U \mid X}^{\varphi}\right) \simeq \mathbb{C}_{X}^{\mathrm{E}} \stackrel{+}{\otimes} \mathbb{C}_{\{t=-\operatorname{Re} \varphi\}} .
$$


We shall apply this result in the following situation.

Let $\mathbb{V}$ be a complex finite-dimensional vector space of complex dimension $d_{\mathbb{V}}, \mathbb{V}^{*}$ its dual. Since $\mathbb{V}$ is a complex vector space, we shall identify or $\mathbb{V}$ with $\mathbb{C}$. We denote here by $\overline{\mathbb{V}}$ the projective compactification of $\mathbb{V}$, we set $\mathbb{H}=\overline{\mathbb{V}} \backslash \mathbb{V}$, and similarly with $\overline{\mathbb{V}}^{*}$ and $\mathbb{H}^{*}$. We also introduce the bordered spaces

$$
\mathbb{V}_{\infty}=(\mathbb{V}, \overline{\mathbb{V}}), \quad \mathbb{V}_{\infty}^{*}=\left(\mathbb{V}^{*}, \overline{\mathbb{V}}^{*}\right)
$$

We set for short

$$
X=\overline{\mathbb{V}} \times \overline{\mathbb{V}}^{*}, U=\mathbb{V} \times \mathbb{V}^{*}, Y=X \backslash U .
$$

We shall consider the function

$$
\phi: \mathbb{V} \times \mathbb{V}^{*} \rightarrow \mathbb{C}, \quad \phi(x, y)=\langle x, y\rangle .
$$

We introduce the Laplace kernel

$$
\mathscr{L}:=\mathscr{E}_{U \mid X}^{\langle x, y\rangle} .
$$

Recall that the kernel of the enhanced Fourier transform with respect to the underlying real vector spaces of $\mathbb{V}$ and $\mathbb{V}^{*}$ is given by

$$
L_{\mathbb{V}}:=\mathbb{C}_{\{t=\operatorname{Re}\langle x, y\rangle\}} \in \mathrm{E}^{\mathrm{b}}\left(\mathrm{I}_{\mathbb{V}_{\infty} \times \mathbb{V}_{\infty}^{*}}\right) .
$$

Also recall that we set $L_{\mathbb{V}}^{a}:=\mathbb{C}_{\{t=-\operatorname{Re}\langle x, y\}\}}$.

Lemma 8.3.1. One has the isomorphism in $\mathrm{E}^{\mathrm{b}}\left(\mathrm{IC}_{X}\right)$

$$
\mathcal{S}_{o l}^{\mathrm{E}}(\mathscr{L}) \simeq \mathbb{C}_{X}^{\mathrm{E}} \stackrel{+}{\otimes} \mathrm{E} j_{! !} L_{\mathbb{V}}^{a},
$$

where $j: \mathbb{V}_{\infty} \times \mathbb{V}_{\infty}^{*} \rightarrow X$ is the inclusion.

Proof. This follows immediately from isomorphism (8.3.1). Q.E.D.

In the sequel, we denote by $D_{\mathbb{V}}$ the Weyl algebra $\Gamma\left(\overline{\mathbb{V}} ; \mathscr{D}_{\overline{\mathbb{V}}}(* \mathbb{H})\right)$ associated with $\mathbb{V}$. We also use the $\left(D_{\mathbb{V} \times \mathbb{V}^{*}}, D_{\mathbb{V}^{*}}\right)$-bimodule $D_{\mathbb{V} \times \mathbb{V}^{*} \rightarrow \mathbb{V}^{*}}$ similar to the bimodule $\mathscr{D}_{X \rightarrow Y}$ in the theory of D-modules, and finally we denote by $\mathrm{O}_{\mathbb{V}}$ the ring of polynomials on $\mathbb{V}$.

The next result is well-known and goes back to [KL 85] or before. 
Lemma 8.3.2. There is a natural isomorphism

$$
\mathscr{D}_{\overline{\mathbb{V}}}(* \mathbb{H}) \stackrel{\mathrm{D}}{\circ} \mathscr{L} \simeq \mathscr{D}_{\overline{\mathbb{V}}^{*}}\left(* \mathbb{H}^{*}\right) \otimes \operatorname{det} \mathbb{V}^{*}
$$

Here, $\operatorname{det} \mathbb{V}^{*}=\bigwedge^{n} \mathbb{V}^{*}$.

Proof. Using the GAGA principle, we may replace $\mathscr{D}_{\overline{\mathbb{V}}}(* \mathbb{H})$ with $\mathrm{D}_{\mathbb{V}}, \mathscr{D}_{\overline{\mathbb{V}}^{*}}(* \mathbb{H})$ with $\mathrm{D}_{\mathbb{V}^{*}}, \mathscr{L}$ with $\mathrm{D}_{\mathbb{V} \times \mathbb{V}^{*}} \mathrm{e}^{\langle x, y\rangle}$ and thus $\mathscr{D}_{\overline{\mathbb{V}}}(* \mathbb{H}) \stackrel{\mathrm{D}}{\circ} \mathscr{L}$ with

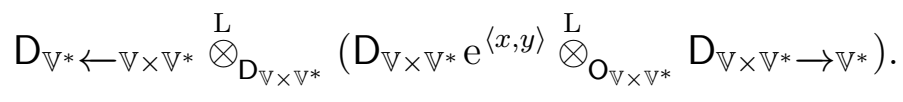

This last object is isomorphic to

$$
\left(D_{\mathbb{V}^{*} \leftarrow \mathbb{V} \times \mathbb{V}^{*}} \stackrel{\mathrm{L}}{\mathrm{O}_{\mathrm{V} \times \mathbb{V}^{*}}} \mathrm{D}_{\mathbb{V} \times \mathbb{V}^{*} \rightarrow \mathbb{V}}\right) \stackrel{\mathrm{L}}{\otimes_{\mathscr{D}_{\mathbb{V} \times \mathbb{V}^{*}}}} \mathrm{D}_{\mathbb{V} \times \mathbb{V}^{*}} \mathrm{e}^{\langle x, y\rangle} .
$$

Since

$$
\mathrm{D}_{\mathbb{V}^{*} \leftarrow \mathbb{V} \times \mathbb{V}^{*}} \stackrel{\mathrm{L}}{\otimes_{\mathrm{O}_{\mathbb{V} \times \mathbb{V}^{*}}}} \mathrm{D}_{\mathbb{V} \times \mathbb{V}^{*} \rightarrow \mathbb{V}} \simeq D_{\mathbb{V} \times \mathbb{V}^{*}} \otimes \operatorname{det} \mathbb{V}^{*},
$$

the module (8.3.5) is isomorphic to $D_{\mathbb{V} \times \mathbb{V}^{*}} \mathrm{e}^{\langle x, y\rangle} \otimes \operatorname{det} \mathbb{V}^{*}$. Finally, one remarks that the natural morphism $\mathrm{D}_{\mathbb{V}^{*}} \rightarrow \mathrm{D}_{\mathbb{V} \times \mathbb{V}^{*}} \mathrm{e}^{\langle x, y\rangle}$ is an isomorphism. Q.E.D.

In the sequel, we shall identify $\mathrm{D}_{\mathbb{V}}$ and $\mathrm{D}_{\mathbb{V}^{*}}$ by the correspondence $x_{i} \leftrightarrow$ $-\partial_{y_{i}}, \partial_{x_{i}} \leftrightarrow y_{i}$. (Of course, this does not depend on the choice of linear coordinates on $\mathbb{V}$ and the dual coordinates on $\mathbb{V}^{*}$.)

Theorem 8.3.3. We have an isomorphism in $\mathrm{D}^{\mathrm{b}}\left(\left(\mathrm{ID}_{\mathbb{V}}\right)_{\mathbb{V}_{\infty}^{*}}\right)$

$$
\mathrm{E}_{\mathscr{F}_{\mathbb{V}}}\left(\mathscr{O}_{\mathbb{V}_{\infty}}^{\mathrm{E}}\right) \simeq \mathscr{O}_{\mathbb{V}_{\infty}^{*}}^{\mathrm{E}} \otimes \operatorname{det} \mathbb{V}\left[-d_{\mathbb{V}}\right]
$$

Proof. Set $K=\mathcal{S}$ ol $l_{\mathbb{V}_{\infty} \times \mathbb{V}_{\infty}^{*}}(\mathscr{L})$. By Theorem 8.1.3, we have

$$
\Psi_{K}^{\mathrm{E}}\left(\mathcal{D} \mathcal{R}_{\mathbb{V}_{\infty}}^{\mathrm{E}}(\mathscr{M})\right)\left[-d_{\mathbb{V}}\right] \simeq \mathcal{D} \mathcal{R}_{\mathbb{V}_{\infty}^{*}}^{\mathrm{E}}(\mathscr{M} \stackrel{\mathrm{D}}{\circ} \mathscr{L}) .
$$

for any $\mathscr{M} \in \mathrm{D}_{\text {q-good }}^{\mathrm{b}}\left(\mathscr{D}_{X}\right)$ such that $\mathscr{M} \simeq \mathscr{M}(* \mathbb{H})$. By Lemma 8.3.1, $K=$ $\mathbb{C}_{\mathbb{V}_{\infty} \times \mathbb{V}_{\infty}^{*}}^{\mathrm{E}} \stackrel{+}{\otimes} L_{\mathbb{V}}^{a}$, and by Corollary 8.2.4, the functor $\mathscr{E}_{\mathbb{V}}$ is isomorphic to the functor $\Psi_{L_{\mathbb{V}}^{a}}^{\mathrm{E}}\left[-2 d_{\mathbb{V}}\right]$. Since

$$
\mathscr{I h o m}^{+}\left(\mathbb{C}_{\mathbb{V}_{\infty}}^{\mathrm{E}}, \mathcal{D} \mathcal{R}_{\mathbb{V}_{\infty}}^{\mathrm{E}}(\mathscr{M})\right) \simeq \mathcal{D} \mathcal{R}_{\mathbb{V}_{\infty}}^{\mathrm{E}}(\mathscr{M})
$$


we have

$$
\Psi_{K}\left(\mathcal{D} \mathcal{R}_{\mathbb{V}_{\infty}}^{\mathrm{E}}(\mathscr{M})\right)\left[-2 d_{\mathbb{V}}\right] \simeq \Psi_{L_{\mathbb{V}}^{a}}\left(\mathcal{D} \mathcal{R}_{\mathbb{V}_{\infty}}^{\mathrm{E}}(\mathscr{M})\right)\left[-2 d_{\mathbb{V}}\right] \simeq \mathscr{F}_{\mathbb{V}}\left(\mathcal{D} \mathcal{R}_{\mathbb{V}_{\infty}}^{\mathrm{E}}(\mathscr{M})\right)
$$

Therefore, we obtain

$$
\mathrm{E}_{\mathbb{V}}\left(\mathcal{D} \mathcal{R}_{\mathbb{V}_{\infty}}^{\mathrm{E}}(\mathscr{M})\right) \simeq \mathcal{D} \mathcal{R}_{\mathbb{V}_{\infty}^{*}}^{\mathrm{E}}(\mathscr{M} \stackrel{\mathrm{D}}{\circ} \mathscr{L})\left[-d_{\mathbb{V}}\right]
$$

Now choose $\mathscr{M}=\mathscr{D}_{\overline{\mathbb{V}}}(* \mathbb{H})$ and apply Lemma 8.3.2. Since $\mathcal{D} \mathcal{R}_{\mathbb{V}_{\infty}}^{\mathrm{E}}(\mathscr{M}) \simeq \Omega_{\mathbb{V}_{\infty}}^{\mathrm{E}}$ and $\mathcal{D} \mathcal{R}_{\mathbb{V}_{\infty}^{*}}^{\mathrm{E}}(\mathscr{M} \stackrel{\mathrm{D}}{\circ} \mathscr{L}) \simeq \Omega_{\mathbb{V}_{\infty}^{*}}^{\mathrm{E}} \otimes \operatorname{det} \mathbb{V}^{*}$, we obtain

$$
\mathrm{E}_{\mathscr{F}_{\mathbb{V}}}\left(\Omega_{\mathbb{V}_{\infty}}^{\mathrm{E}}\right) \simeq \Omega_{\mathbb{V}_{\infty}^{*}}^{\mathrm{E}} \otimes \operatorname{det} \mathbb{V}^{*}\left[-d_{\mathbb{V}}\right]
$$

Hence, it is enough to remark that

$$
\Omega_{\mathbb{V}_{\infty}}^{\mathrm{E}} \simeq \mathscr{O}_{\mathbb{V}_{\infty}}^{\mathrm{E}} \otimes \operatorname{det} \mathbb{V}^{*} \text { and } \Omega_{\mathbb{V}_{\infty}^{*}}^{\mathrm{E}} \simeq \mathscr{O}_{\mathbb{V}_{\infty}^{*}}^{\mathrm{E}} \otimes \operatorname{det} \mathbb{V}
$$

Q.E.D.

Remark 8.3.4. (i) Symbolically, isomorphism (8.3.6) is given by

$$
\mathscr{O}_{\mathbb{V}_{\infty}^{*}}^{\mathrm{E}} \otimes \operatorname{det} \mathbb{V} \ni \phi(y) \otimes d y \longmapsto \int \mathrm{e}^{\langle x, y\rangle} \phi(y) d y \in \mathcal{F}_{\mathbb{V}}\left(\mathscr{O}_{\mathbb{V}_{\infty}}^{\mathrm{E}}\right)
$$

(ii) The identification of $D_{\mathbb{V}}$ and $D_{\mathbb{V}^{*}}$ is given by:

$$
\begin{aligned}
\mathrm{D}_{\mathbb{V}} \ni P\left(x, \partial_{x}\right) \leftrightarrow Q\left(y, \partial_{y}\right) \in \mathrm{D}_{\mathbb{V}^{*}} & \Longleftrightarrow P\left(x, \partial_{x}\right) \mathrm{e}^{\langle x, y\rangle}=Q^{*}\left(y, \partial_{y}\right) \mathrm{e}^{\langle x, y\rangle} \\
& \Longleftrightarrow P^{*}\left(x, \partial_{x}\right) \mathrm{e}^{-\langle x, y\rangle}=Q\left(y, \partial_{y}\right) \mathrm{e}^{-\langle x, y\rangle} .
\end{aligned}
$$

Here $Q^{*}\left(y, \partial_{y}\right)$ denotes the formal adjoint operator of $Q\left(y, \partial_{y}\right) \in \mathrm{D}_{\mathbb{V}^{*}}$.

Applying Corollary 8.2.5, we get:

Corollary 8.3.5. Isomorphism (8.3.4) together with the enhanced FourierSato isomorphism induce an isomorphism in $\mathrm{D}^{\mathrm{b}}\left(\mathrm{D}_{\mathbb{V}}\right)$, functorial in $F \in$ $\mathrm{E}^{\mathrm{b}}\left(\mathrm{I}_{\mathbb{V}_{\infty}}\right)$ :

$$
\operatorname{RHom}^{\mathrm{E}}\left(F, \mathscr{O}_{\mathbb{V}_{\infty}}^{\mathrm{E}}\right) \simeq \operatorname{RHom}^{\mathrm{E}}\left(\mathscr{F}_{\mathbb{V}}(F), \mathscr{O}_{\mathbb{V}_{\infty}^{*}}^{\mathrm{E}}\right) \otimes \operatorname{det} \mathbb{V}\left[-d_{\mathbb{V}}\right]
$$

As a consequence of Corollary 8.3.5, we recover the main result of [KS 97]: 
Corollary 8.3.6. Isomorphism (8.3.4) together with the Fourier-Sato isomorphism induces an isomorphism in $\mathrm{D}^{\mathrm{b}}\left(\mathrm{D}_{\mathbb{V}}\right)$, functorial in $G \in \mathrm{D}_{\mathbb{R}^{+}}^{\mathrm{b}}\left(\mathbb{C}_{\mathbb{V}}\right)$ :

$$
\operatorname{RHom}\left(G, \mathscr{O}_{\mathbb{V}_{\infty}}^{\mathrm{t}}\right) \simeq \operatorname{RHom}\left(\mathrm{S}_{\mathscr{F}_{\mathbb{V}}}(G), \mathscr{O}_{\mathbb{V}_{\infty}^{*}}^{\mathrm{t}}\right) \otimes \operatorname{det} \mathbb{V}\left[-d_{\mathbb{V}}\right]
$$

Here, letting $a_{\mathbb{V}_{\infty}}: \mathbb{V}_{\infty} \rightarrow$ pt be the projection,

$$
\operatorname{RHom}\left(G, \mathscr{O}_{\mathbb{V}_{\infty}}^{\mathrm{t}}\right):=\alpha_{\mathrm{pt}} \operatorname{R} a_{\mathrm{V}_{\infty} *} \operatorname{R} \mathscr{I h o m}{ }_{\mathrm{IC}_{\mathbb{V}_{\infty}}}\left(G, \mathscr{O}_{\mathbb{V}_{\infty}}^{\mathrm{t}}\right) \in \mathrm{D}^{\mathrm{b}}(\mathbb{C}) .
$$

Proof. By Theorem 8.2.6, we have $\mathrm{E}_{\mathbb{F}_{\mathbb{V}}}\left(\epsilon_{\mathbb{V}_{\infty}}(G)\right) \simeq \epsilon_{\mathbb{V}_{\infty}^{*}} \mathrm{~S} \mathscr{F}_{\mathbb{V}}(G)$, where $\epsilon_{\mathbb{V}_{\infty}}$ is given in (8.2.1). Applying isomorphism (8.3.7) with $F=\epsilon_{\mathbb{V}_{\infty}}(G)$, we obtain

$$
\operatorname{RHom}^{\mathrm{E}}\left(\epsilon_{\mathbb{V}_{\infty}}(G), \mathscr{O}_{\mathbb{V}_{\infty}}^{\mathrm{E}}\right) \simeq \operatorname{RHom}^{\mathrm{E}}\left(\epsilon_{\mathbb{V}_{\infty}^{*}} \mathrm{~S}_{\mathscr{F}_{\mathbb{V}}}(G), \mathscr{O}_{\mathbb{V}_{\infty}^{*}}^{\mathrm{E}}\right) \otimes \operatorname{det} \mathbb{V}\left[-d_{\mathbb{V}}\right]
$$

We have

$$
\begin{aligned}
\operatorname{RHom}^{\mathrm{E}}\left(\epsilon_{\mathbb{V}_{\infty}}(G), \mathscr{O}_{\mathbb{V}_{\infty}}^{\mathrm{E}}\right) & \simeq \alpha_{\mathrm{pt}} \operatorname{R} a_{\mathbb{V}_{\infty *}} \mathscr{I h o m}^{\mathrm{E}}\left(\epsilon_{\mathbb{V}_{\infty}}(G), \mathscr{O}_{\mathbb{V}_{\infty}}^{\mathrm{E}}\right) \\
& \simeq \alpha_{\mathrm{pt}} \operatorname{R} a_{\mathbb{V}_{\infty *}} \operatorname{R} \mathscr{I} h o m\left(G, \mathscr{O}_{\mathbb{V}_{\infty}}^{\mathrm{t}}\right) \\
& \simeq \operatorname{RHom}\left(G, \mathscr{O}_{\mathbb{V}_{\infty}}^{\mathrm{t}}\right),
\end{aligned}
$$

and similarly $\operatorname{RHom}^{\mathrm{E}}\left(\epsilon_{\mathbb{V}_{\infty}^{*}} \mathrm{~S} \mathscr{F}_{\mathbb{V}}(G), \mathscr{O}_{\mathbb{V}_{\infty}^{*}}^{\mathrm{E}}\right) \simeq \operatorname{RHom}\left(\mathscr{S}_{\mathbb{V}}(G), \mathscr{O}_{\mathbb{V}_{\infty}^{*}}^{\mathrm{t}}\right)$. Q.E.D.

\section{References}

[BM88] E. Bierstone and P.-D. Milman, Semi-analytic sets and subanalytic sets, Inst. Hautes Études Sci. Publ. Math. 67 (1988) 5-42.

[Bj 93] J-E. Björk, Analytic $\mathscr{D}$-modules and Applications, Kluwer Academic Publisher, Dordrecht-Boston-London (1993) xiv+581 pp.

[BE04] S. Bloch and H. Esnault, Homology for irregular connections, J. Théor. Nombres Bordeaux 16 (2004) 357-371.

[DK13] A. D'Agnolo and M. Kashiwara, Riemann-Hilbert correspondence for irregular holonomic systems, arXiv:1311.2374v1.

[DS96] A. D'Agnolo and P. Schapira, Leray's quantization of projective duality, Duke Math. J. 84 no. 2 (1996) 453-496.

[De70] P. Deligne, Equations différentielles à points singuliers réguliers, Lecture Notes in Math. 163 Springer (1970). 
[Ga81] O. Gabber, The integrability of the characteristic variety, Amer. Journ. Math. 103 (1981) 445-468.

[Gr66] A. Grothendieck, On the de Rham cohomology of algebraic varieties, Inst. Hautes Études Sci. Publ. Math. 29 (1966) 93-101.

[GS12] S. Guillermou and P. Schapira, Microlocal theory of sheaves and Tamarkin's non displaceability theorem, Homological Mirror Symmetry and Tropical Geometry, LN of the UMI, Springer pp. 43-85 (2014), arXiv:1106.1576v2 (2012).

[Hi09] M. Hien, Periods for flat algebraic connections, Invent. Math. 178 (2009), 1-22.

[HTT08] R. Hotta, K. Takeuchi and T. Tanisaki, D-modules, Perverse Sheaves, and Representation Theory, Progress in Math. 236 Birkhäuser (2008) $\mathrm{x}+407 \mathrm{pp}$.

[Ka70] M. Kashiwara, Algebraic study of systems of partial differential equations, Thesis, Tokyo Univ. (1970), Mémoires Soc. Math. France (N,S.) 63 (1995) xiv+72 pp.

[Ka75] M. Kashiwara, On the maximally overdetermined system of linear differential equations I, Publ. Res. Inst. Math. Sci. 10 (1974/75), 563579 .

[Ka78] M. Kashiwara, On the holonomic systems of linear differential equations II, Invent. Math. 49 (1978), 121-135.

[Ka80] _ Faisceaux constructibles et systèmes holonômes d'équations aux dérivées partielles linéaires à points singuliers réguliers, Séminaire Goulaouic-Schwartz, 1979-1980, Exp. No. 19 École Polytech., Palaiseau, 1980 .

[Ka 84] - The Riemann-Hilbert problem for holonomic systems, Publ. Res. Inst. Math. Sci. 20 no. 2 (1984) 319-365.

[Ka03] _ D-modules and Microlocal Calculus, Translations of Mathematical Monographs, 217, American Math. Soc. (2003). 
[KK 81] M. Kashiwara and T. Kawai, On holonomic systems of microdifferential equations III. Systems with regular singularities, Publ. Res. Inst. Math. Sci. 17 (1981) 813-979.

[KS 90] M. Kashiwara and P. Schapira, Sheaves on Manifolds, Grundlehren der Math. Wiss. 292 Springer-Verlag (1990).

[KS96] - Moderate and formal cohomology associated with constructible sheaves, Mem. Soc. Math. France 64 (1996).

[KS97]__ Integral transforms with exponential kernels and Laplace transform, Journal of the AMS 10 (1997) 939-972.

[KS 01] — Ind-sheaves, Astérisque 271 (2001), 136 pp.

[KS03] _ Microlocal study of ind-sheaves I. Micro-support and regularity, Astérisque 284 (2003) 143-164.

[KS 06] , Categories and Sheaves, Grundlehren der Math. Wiss. 332 Springer-Verlag (1990) x+512 pp.

[KS14] _ Irregular holonomic kernels and Laplace transform, to appear in Selecta Mathematica, arXiv:1402.3642v1.

[KS15] _ Lectures on Regular and Irregular Holonomic D-modules, (2015), http://preprints.ihes.fr/2015/M/M-15-08.pdf.

[KL 85] N. Katz and G. Laumon, Transformation de Fourier et majoration de sommes d'exponentielles, Inst. Hautes Études Sci. Publ. Math. 63 (1985) 361-418.

[Ke10] K. S. Kedlaya, Good formal structures for flat meromorphic connections, I: surfaces, Duke Math. J. 154 no. 2 (2010) 343-418.

[Ke11] _ Good formal structures for flat meromorphic connections, II: Excellent schemes, J. Amer. Math. Soc. 24 (2011) 183-229.

[Lo59] S. Lojasiewicz, Sur le problème de la division, Studia Math. 8, (1959) $87-136$.

[Ma66] B. Malgrange, Ideals of Differentiable Functions, Tata Institute of Fundamental Research Studies in Mathematics, No. 3, Oxford University Press, London (1967) vii+106 pp. 
[Me84] Z. Mebkhout, Une équivalence de catégories-Une autre équivalence de catégories, Comp. Math., 51 (1984) 55-62 and 63-98.

[Mo09] T. Mochizuki, Good formal structure for meromorphic flat connections on smooth projective surfaces, Algebraic analysis and around, Adv. Stud. Pure Math., 54, Math. Soc. Japan, Tokyo, (2009) 223-253.

[Mo11] Wild Harmonic Bundles and Wild Pure Twistor DD-modules, Astérisque 340 (2011) x+607 pp.

[Mr10] G. Morando, Preconstructibility of tempered solutions of holonomic $\mathscr{D}$-modules, Int. Math. Res. Not. (2014), no. 4, 1125-1151, arXiv: 1007.4158.

[Po74] J. B. Poly, Sur l'homologie des courants à support dans un ensemble semi-analytique, Journnées de géométrie analytique (Univ. Poitiers, 1972), pp. 35-43. Bull. Soc. Math. Suppl. Mem., 38, Soc. Math. France, Paris, 1974.

[Pr08] L. Prelli, Sheaves on subanalytic sites, Rend. Semin. Mat. Univ. Padova, 120 (2008), 167-216.

[Ra78] J-P. Ramis, Additif II à "variations sur le thème GAGA", Lecture Notes in Math. 694 Springer (1978), 280-289.

[Sa00] C. Sabbah, Équations différentielles à points singuliers irréguliers et phénomène de Stokes en dimension 2, Astérisque 263 (2000) viii+190 pp.

[SKK 73] M. Sato, T. Kawai and M. Kashiwara, Microfunctions and pseudodifferential equations, in Komatsu (ed.), Hyperfunctions and pseudodifferential equations, Proceedings Katata 1971, Lecture Notes in Math. Springer-Verlag 287 (1973) 265-529.

[Sc 86] J-P. Schneiders, Un théorème de dualité pour les modules différentiels, C. R. Acad. Sci. 303 (1986) 235-238.

[Sc99] _ Quasi-abelian Categories and Sheaves, Mem. Soc. Math. France 76 (1999) 134 pp. 
[SGA 4] S-G-A 4, Sém. Géom. Alg. (1963-64) by M. Artin, A. Grothendieck and J-L. Verdier, Théorie des topos et cohomologie étale des schémas, Lecture Notes in Math. 269, 270, 305 (1972/73).

[Ta08] D. Tamarkin, Microlocal condition for non-displaceability, arXiv:0809.1584v1.

[VD 98] L. Van den Dries, Tame Topology and O-minimal Structures, London Math. Soc. Lect. Notes Series, 248 (1998) x+180 pp. 


\section{List of Notations}

\begin{tabular}{|c|c|}
\hline$(M, \widehat{M}), 56$ & $\begin{array}{l}\mathrm{D} \\
\mathrm{\circ}, 54\end{array}$ \\
\hline$E_{U \mid X}^{\varphi}, 78$ & $\mathbb{D}, 11$ \\
\hline$F^{\mathrm{E}}, 71$ & $\mathrm{D} f_{!}, 12$ \\
\hline$L \circ, 54$ & $\Delta_{M}, 90$ \\
\hline$I \mathrm{E}$ & $\mathrm{D}^{\mathrm{b}}\left(\mathbf{k}_{M}\right), 7$ \\
\hline$L \circ, 98$ & $\mathrm{D}^{\mathrm{b}}\left(\mathrm{Ik}_{(M, \widehat{M})}\right), 57$ \\
\hline$L_{\mathrm{V}}^{a}, 99$ & $\mathrm{D}_{\mathrm{IR}-\mathrm{c}}^{\mathrm{b}}\left(\mathrm{Ik}_{M}\right), 27$ \\
\hline$L_{\mathrm{V}^{*}}^{a}, 99$ & $\mathrm{D}_{\mathbb{R}-\mathrm{c}}^{\mathrm{b}}\left(\mathbf{k}_{M}\right), 9$ \\
\hline$L_{\mathrm{V}}, 99$ & $\mathrm{D}_{\mathrm{rh}}^{\mathrm{b}}\left(\mathscr{D}_{X}\right), 44$ \\
\hline $\mathcal{A}_{\widetilde{X}}, 47$ & \\
\hline $\mathrm{D}_{\mathbb{R}-\mathrm{c}}^{\mathrm{b}}\left(\mathbf{k}_{M \times \mathbb{R}_{\infty}}\right), 73$ & $\bigotimes, 12$ \\
\hline $\mathrm{D}_{\mathrm{coh}}^{\mathrm{b}}\left(\mathscr{D}_{X}\right), 11$ & $\mathrm{D} f_{*}, 12$ \\
\hline $\mathrm{D}_{\text {good }}^{\mathrm{b}}\left(\mathscr{D}_{X}\right), 11$ & $\mathrm{D} f^{*}, 12$ \\
\hline $\mathrm{D}_{\mathrm{hol}}^{\mathrm{b}}\left(\mathscr{D}_{X}\right), 11$ & $\mathrm{D}_{\mathbb{V}}, 101$ \\
\hline $\mathrm{D}_{\mathrm{q}-\operatorname{good}}^{\mathrm{b}}\left(\mathscr{D}_{X}\right), 11$ & $\otimes, 11,77$ \\
\hline $\mathbb{C}_{\{\operatorname{Re} \varphi<*\}}, 78$ & $\mathrm{E} \mathscr{F} \mathrm{V}, 99$ \\
\hline $\mathcal{C}_{M}^{\infty}, 29$ & $\mathrm{E} \mathscr{F}_{\mathrm{V}^{*}}^{a}, 99$ \\
\hline $\mathcal{C}_{M}^{\omega}, 29$ & $\mathrm{D}_{M}^{\mathrm{E}}, 73$ \\
\hline $\mathcal{C}_{M}^{\infty, \mathrm{t}}, 30$ & RHom $^{\mathrm{E}}, 67$ \\
\hline $\mathcal{C}_{M_{\mathrm{sa}}}^{\infty, \mathrm{t}}, 30$ & $\mathrm{FN}, 35$ \\
\hline $\mathcal{C}_{M}^{\infty, \mathrm{w}}, 30$ & $\mathrm{I}(\mathcal{A}), 21$ \\
\hline $\mathcal{C}_{M_{\mathrm{sa}}}^{\infty, \mathrm{w}}, 30$ & $\mathrm{I}(\mathbf{k}), 16$ \\
\hline $\mathscr{D} \widetilde{\widetilde{A}}, 47$ & $\mathrm{I}\left(\mathbf{k}_{M}\right), 17$ \\
\hline $\mathrm{DFN}, 35$ & $\mathrm{I}_{\mathbb{R}-\mathrm{c}}\left(\mathbf{k}_{M}\right), 25$ \\
\hline $\mathscr{D}_{X} \mathrm{e}^{\varphi}, 77$ & $M_{\mathrm{sa}}, 24$ \\
\hline $\mathscr{D}_{X}, 10$ & $\mathscr{O}_{X}^{\mathrm{E}}, 79$ \\
\hline $\mathscr{D}_{M \rightarrow N}, 31$ & $\mathscr{O}_{X}(* S), 42$ \\
\hline $\mathscr{D}_{X \leftarrow Y}, 12$ & $\Omega_{X}, 10$ \\
\hline $\mathscr{D}_{X} \rightarrow Y, 12$ & $\Omega_{X / Y}, 10$ \\
\hline $\mathcal{D} b_{M}^{\top}, 78$ & $\mathrm{Op}_{M}, 24$ \\
\hline $\mathcal{D} b_{M}, 29$ & $\mathrm{Op}_{M_{\mathrm{sa}}}, 24$ \\
\hline $\mathcal{D} b_{M}^{\mathrm{t}}, 30$ & $\mathscr{O}_{X}^{\mathrm{t}}, 34$ \\
\hline $\mathcal{D} b_{M_{\mathrm{sa}}}^{\mathrm{t}}, 30$ & $\mathscr{O}_{\widetilde{Y}}^{\mathrm{t}}, 47$ \\
\hline $\mathcal{D} b_{M}^{\mathrm{tV}}, 31$ & $\Omega_{X}^{\mathrm{E}}, 79$ \\
\hline
\end{tabular}


$\Omega_{X}^{\mathrm{t}}, 34$

$\Omega_{\widetilde{X}}^{\mathrm{t}}, 48$

$\mathscr{O}_{X}^{\mathrm{W}}, 34$

$\mathscr{O}_{X}^{\omega}, 34$

$\Phi_{L}, 54$

$\Phi_{L}^{\mathrm{E}}, 98$

$\Psi_{L}, 54$

$\mathrm{D}_{M}^{\prime}, 8$

$\mathrm{D}_{M}, 8$

$\mathbb{R}_{\infty}, 62$

$\mathcal{I}\left(\mathbf{k}_{M}\right), 17$

$\operatorname{SingSupp}(\mathscr{M}), 44$

$\operatorname{Supp}(F), 7$

$\mathrm{E}^{\mathrm{b}}\left(\mathbf{k}_{M}\right), 66$

$\mathrm{E}^{\mathrm{b}}\left(\mathrm{Ik}_{M}\right), 65$

$\mathrm{E}_{ \pm}^{\mathrm{b}}\left(\mathrm{Ik}_{M}\right), 65$

$\mathrm{E}_{\mathbb{R}-\mathrm{c}}^{\mathrm{b}}\left(\mathrm{Ik}_{M}\right), 73$

$\mathrm{E} f ! !, 69$

$\mathrm{E} f !, 69$

$\Theta_{M}, 31$

$\Theta_{X}, 10$

$\mathrm{L}^{\mathrm{E}}, 67$

$\mathrm{E} f_{*}, 69$

$\mathrm{E} f^{-1}, 69$

$\mathrm{R}^{\mathrm{E}}, 67$

$\alpha_{M}, 17$

$\overline{\mathrm{V}}, 99$

$\beta_{M}, 17$

古, 69

$\operatorname{char}(\mathscr{M}), 10$

$\mathscr{I h o m}^{+}, 63,68$

$\mathscr{I h o m}_{\beta \mathcal{A}}^{+}, 77$

$\mathbf{k}_{M}^{\mathrm{E}}, 71$

$\mathbf{k}_{\{t<*\}}, 71$

$\mathbf{k}_{\{t>*\}}, 75$

$\mathbf{k}_{\{t \gg 0\}}, 71$ $\stackrel{+}{\otimes}, 63,68$

$\stackrel{+}{\otimes}_{\beta \mathcal{A}}, 77$

$\operatorname{dim} M, 31$

$\mathcal{D R}_{X}^{\mathrm{E}}, 80$

$\mathcal{D} \mathcal{R}_{\widetilde{X}}^{\mathrm{E}}, 88$

$\mathcal{D} \mathcal{R}_{X}, 13$

$\mathcal{D} \mathcal{R}_{X}^{\mathrm{t}}, 38$

$\tilde{f}^{!}, 69$

$f^{!}, 8,20,58$

$\epsilon_{M}, 73$

$\epsilon_{M_{\infty}}, 98$

$\otimes, 8$

$\mathscr{H}_{0 m^{\mathrm{E}}}, 67$

$\mathscr{I h o m}^{\mathrm{E}}, 67$

Ihom, 17

$\mathrm{IC}_{t^{*}=0}, 65$

$\mathrm{IC}_{t^{*}>0}, 65$

$\mathrm{IC}_{t^{*} \leq 0}, 65$

$\operatorname{Ind}(\mathscr{C}), 15$

$\iota_{M}, 18$

$\operatorname{Mod}\left(\mathscr{D}_{X}^{\text {op }}\right), 10$

$\operatorname{Mod}\left(\mathscr{D}_{X}\right), 10$

$\operatorname{Mod}(\mathbf{k}), 16$

$\operatorname{Mod}\left(\mathbf{k}_{M}\right), 7$

$\operatorname{Mod}_{\operatorname{coh}}\left(\mathscr{D}_{X}\right), 10$

$\operatorname{Mod}^{\mathrm{c}}\left(\mathbf{k}_{M}\right), 17$

$\operatorname{Mod}^{f}(\mathbf{k}), 16$

$\operatorname{Mod}_{\mathrm{rh}}\left(\mathscr{D}_{X}\right), 44$

$\mu\left(t_{1}, t_{2}\right), 62$

$\overline{\mathbb{R}}, 62$

$\omega_{M}, 8$

$\tilde{f}^{-1}, 69$

$f^{-1}, 8,20,58$

or $_{M}, 31$

$\mathscr{A}, 31$

$\mathbb{R}-\mathrm{C}\left(\mathbf{k}_{M}\right), 25$

$\mathbb{R}-\mathrm{C}^{\mathrm{c}}\left(\mathbf{k}_{M}\right), 25$ 


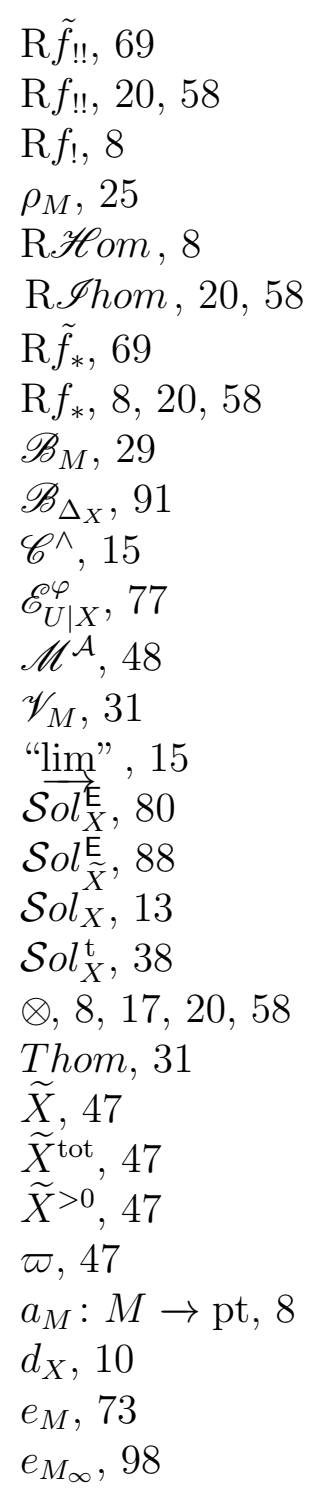




\section{Index}

base change formula for D-modules, 14

for indsheaves, 23

for sheaves, 9

bordered spaces, 56

proper morphism of, 60

D-module, 10

good, 11

holonomic, 10

quasi-good, 10

regular holonomic, 44

de Rham functor, 13

enhanced, 80

tempered, 38

duality

for D-modules, 11

for enhanced indsheaves, 73

dualizing complex, 8

enhanced

Fourier-Sato transform, 98-100

indsheaves, 65

tempered holomorphic functions, 78

exponential D-modules, 77

external tensor product

for D-modules, 12

for enhanced indsheaves, 69

for sheaves, 8

Fourier-Sato transform

enhanced, 98-100

good

D-module, 11 topological space, 7

Grothendieck's six operations for enhanced indsheaves, 68

for indsheaves, 19

for sheaves, 8

holonomic D-module, 10

ind-object, 15

indsheaves, 17

enhanced, 65

of tempered $\mathrm{C}^{\infty}$-functions, 30

of tempered distributions, 30

of tempered holomorphic functions, 34

of Whitney functions, 30

of Whitney holomorphic functions, 34

integral transforms, 97

regular, 54

Laplace

kernel, 101

transform, 100

non-characteristic, 13

normal form, 85

quasi-, 85

regular, 44

polynomial growth, 29

projection formula

for D-modules, 13

for indsheaves, 23

for sheaves, 9

proper morphism 
of bordered spaces, 60

quasi-good D-module, 10

quasi-normal form, 85

$\mathbb{R}$-constructible enhanced indsheaves, 73

sheaves, 9

ramification, 85

real blow up, 46

regular holonomic D-module, 44

Riemann-Hilbert correspondence

enhanced, 96

generalized, 94

generalized regular, 50

regular, 53

solution functor, 13

enhanced, 80

tempered, 38

stable object, 71

Stokes matrices, 83

subanalytic

indsheaf, 25

site, 24

space, 24

subset, 9

tempered

$\mathrm{C}^{\infty}$-functions, 29

distributions, 29

holomorphic functions, 34

tempered Grauert theorem, 40

transfer bimodule, 12

transversal Cartesian diagram, 14

Weyl algebra, 101

Whitney functions, 30 
Masaki Kashiwara

Research Institute for Mathematical Sciences, Kyoto University

Kyoto, 606-8502, Japan

e-mail: masaki@kurims.kyoto-u.ac.jp

Pierre Schapira

Institut de Mathématiques, Université Pierre et Marie Curie and Mathematics Research Unit, University of Luxembourg 4 Place Jussieu, 7505 Paris

e-mail: schapira@math.jussieu.fr

http://www.math.jussieu.fr/ schapira/ 